\title{
STUDY THE EFFECTS OF CORE ORIENTATION AND DIFFERENT FACE THICKNESSES ON MECHANICAL BEHAVIOR OF HONEYCOMB SANDWICH STRUCTURES UNDER THREE POINT BENDING
}

\author{
A Thesis Presented to \\ The Faculty of California Polytechnic State University \\ San Luis Obispo \\ In Partial Fulfillment \\ Of the Requirements for the Degree of \\ Masters of Science in Aerospace Engineering
}

By

Joshua M. Lister

February 2014 
(C) 2014

Joshua M. Lister

\section{ALL RIGHTS RESERVED}




\section{COMMITTEE MEMBERSHIP}

TITLE:

Study the Effect of Core Orientation and Different Face

Thicknesses on Mechanical Behavior of Honeycomb Sandwich

Structures Under Three Point Bending

AUTHOR: $\quad$ Joshua Martyn Lister

DATE SUBMITTED: February 2013

COMMITTEE CHAIR: $\quad$ Faysal Kolkailah, PhD

Professor of Aerospace Engineering

COMMITTEE MEMBER: Eltahry Elghandour, PhD

Professor of Aerospace Engineering

COMMITTEE MEMBER: Eric Mehiel, $\mathrm{PhD}$

Associate Professor of Aerospace Engineering

COMMITTEE MEMBER: Andrew Davol, PhD

Professor of Mechanical Engineering 


\begin{abstract}
Study the Effect of Core Orientation and Different Face Thicknesses on Mechanical Behavior of Honeycomb Sandwich Structures Under Three Point Bending
\end{abstract}

\author{
Joshua M. Lister ${ }^{1}$ \\ California Polytechnic State University, San Luis Obispo, CA, 93407
}

This study will present the Experimental, numerical and analytical characterizations of composite sandwich structures needed to optimize structure design. In this study, the effects of varying honeycomb core ribbon orientation and varying face sheet thickness's have on the flexural behavior of honeycomb sandwich structures was investigated. Honeycomb sandwich panels were constructed using Hexcel 6367 A250-5H carbon fiber face sheets and Hexcel Nomex HRH-10-1/8-5 honeycomb cores. The mechanical properties of the constituent materials were discovered experimentally using ASTM standards and theoretical models using honeycomb mechanics and classical beam and plate theory are described. A failure mode map for loading under three point bending is developed from previous works by Triantafillou and Gibson $^{26}$, showing the dependence of failure mode on face sheet to core thickness and honeycomb core ribbon orientation. Beam specimens are tested with the effects of Honeycomb core ribbon orientation and unequal face sheet thickness's examined. Experimental data sufficiently agrees with theoretical predictions. A finite element model was developed in ABAQUS/CAE to validate experimental and analytical analysis and produced agreeable results. Optimal bending stiffness and strength with respect to minimum weight was analyzed. The results reveal an important role core ribbon orientation has in a sandwich beam's bending behavior, and design of unequal ply count face sheets can produce higher stiffness to weight ratios than conventional symmetric sandwich structures of similar weight when subjected to a single static load. 


\section{ACKNOWLEDGMENTS}

I would like to thank my advisor Dr. Kolkailah and co-advisors Dr. Elghandour, Dr. Mehiel and Dr. Davol for their help and guidance through this project, which was greatly appreciated. A special thank you goes out to Dr. Elghandour for his endurance with helping me in the experimental testing. Also a great thank you to my two good friends and co-workers Jeff Carter and Justin Tafoya on their help and friendship throughout this thesis. I wouldn't have been able to complete this project without them. To my parents for their guidance, love and support throughout this long college career, I know it's been tough, but we are finally through. I'd also like to thank Hexcel inc. for donating the carbon fiber and Nomex honeycomb used in this thesis. 


\section{TABLE OF CONTENTS}

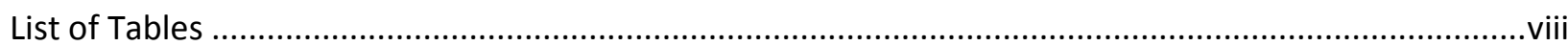

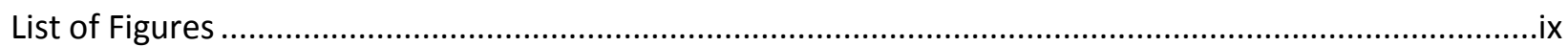

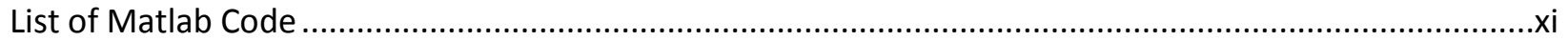

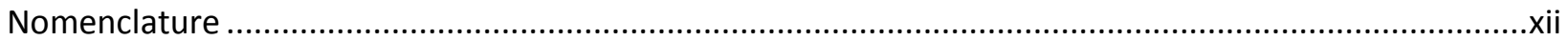

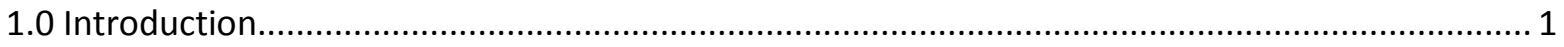

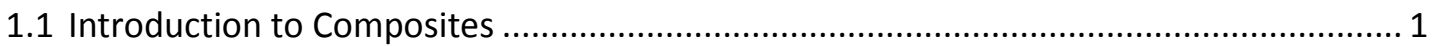

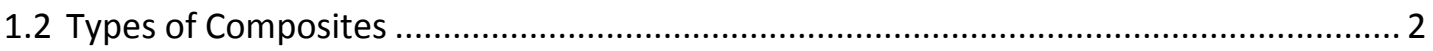

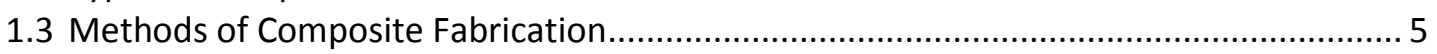

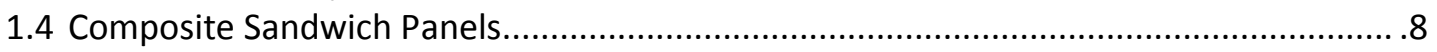

1.4.1 Advantages and Disadvantages of Sandwich Composites........................... 9

1.4.2 Aerospace Industrial Application................................................................. 11

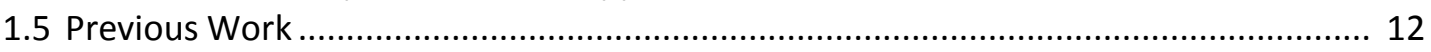

1.6 Main Objective and Scope of Research................................................................ 15

2.0 Manufacturing, Procedure and Experimental Set-up ........................................................ 17

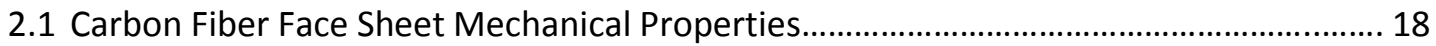

2.1.1 Tensile Mechanical Properties.................................................................. 19

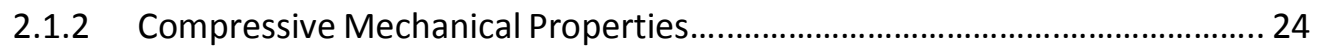

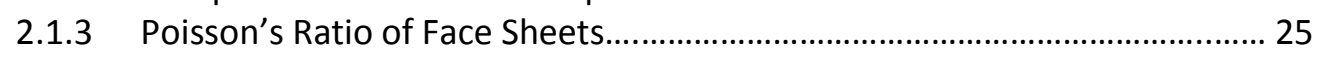

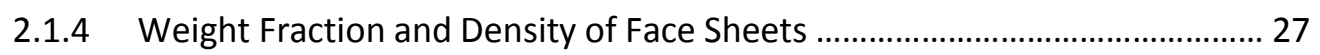

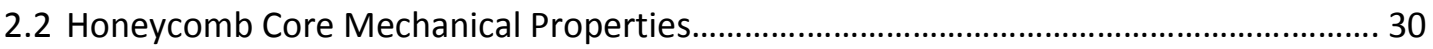

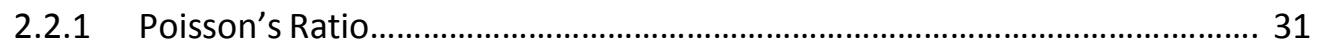

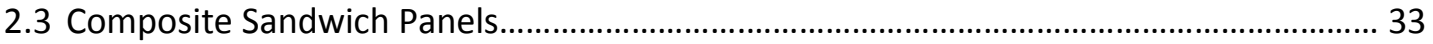

2.3.1 Necessity of Adhesive Layer between Core and Face Sheet......................... 33

2.3.2 Core Shear Properties of Sandwich Constructions by Beam Flexure ......... 35

2.3.3 Determination of Sandwich Beam Flexural and Shear Stiffness ............... 36

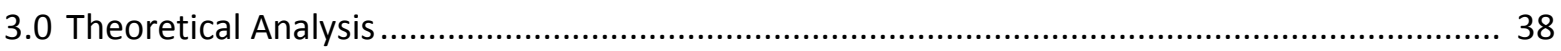

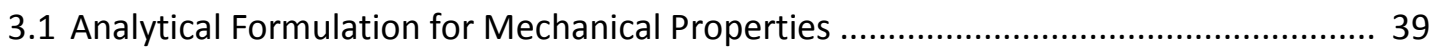

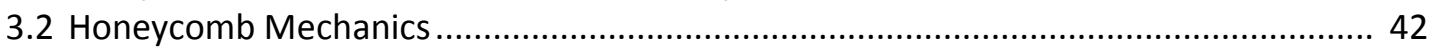

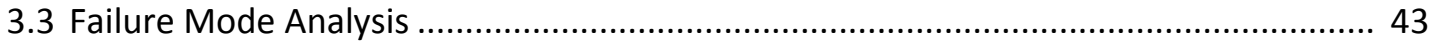

3.4 Theoretical Midspan Deflections of Varying Core Ribbon Orientation.............................. 50

3.5 Theoretical Midpsan Deflections of Varying Face Sheet Thickness's................................ 51

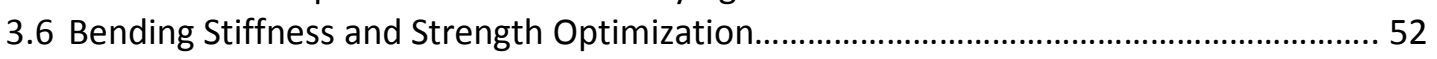

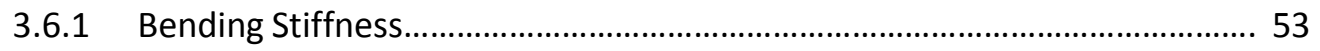

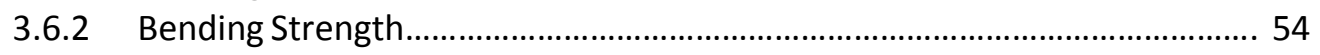

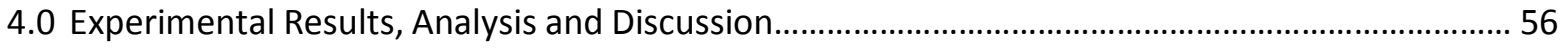

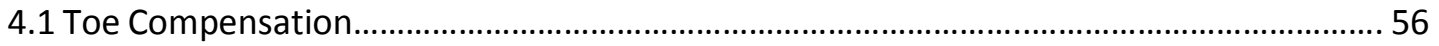

4.2 Experimental Analysis of Sandwiches with Varying Core Orientation .............................. 57

4.3 Experimental Analysis of Sandwiches with Varying Face Sheet Thickness........................ 62

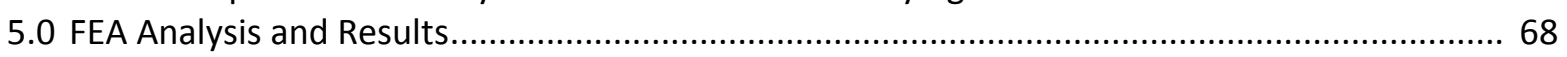

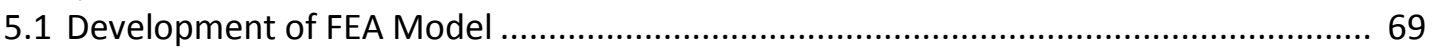

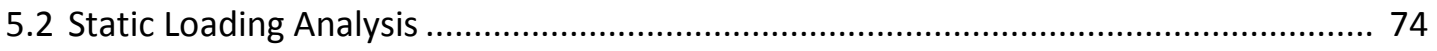

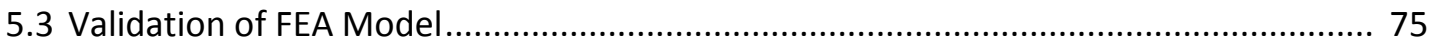

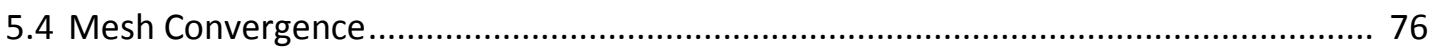

6.0 Comparison of Experimental, Theoretical, and FEA Results .................................................. 78 
6.1 Composite Sandwich Panels with Varying Core Ribbon Orientation ............................ 78

6.2 Composite Sandwich Panels with Varying Face Sheet Thickness ................................ 81

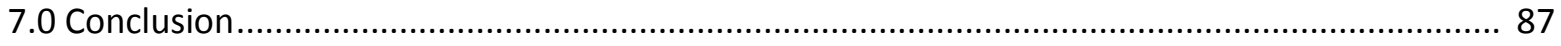

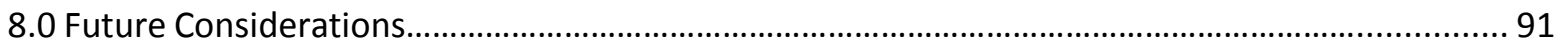

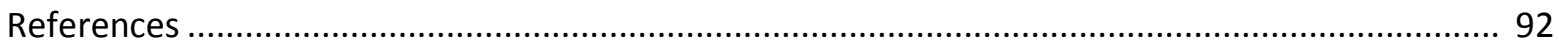

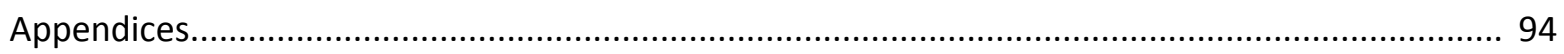

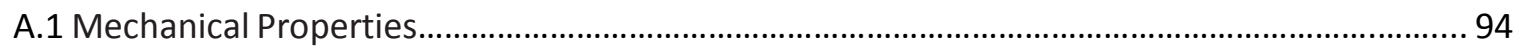

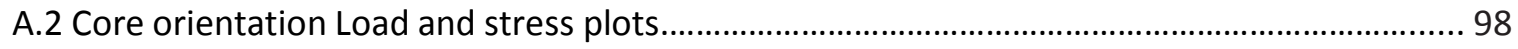

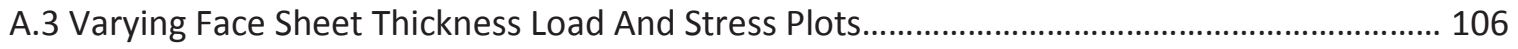

A.4 Finite Element Analysis - Core Ribbon Direction................................................................. 131

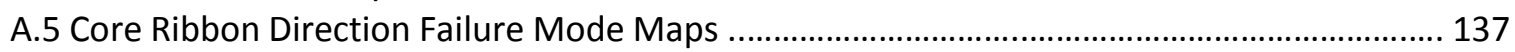

A.6 Varying Face Sheet thicknesses Failure Loads .................................................................... 140

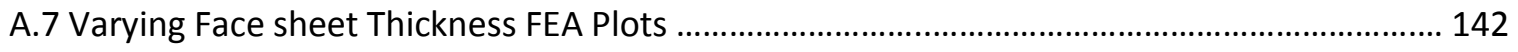

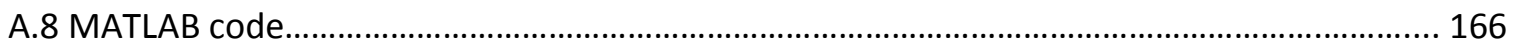




\section{LIST OF TABLES}

Table 1.1. An example of sandwich panel structural efficiency with respect to weight.............................. 10

Table 2.1. ASTM standards used in this experiment.............................................................................. 17

Table 2.2. Tensile mechanical properties of Hexply 6376 A280-5H Carbon Fiber pre-preg....................... 23

Table 2.3. Compressive Mechanical properties of Hexply 6376 A280-5H Carbon Fiber.............................. 25

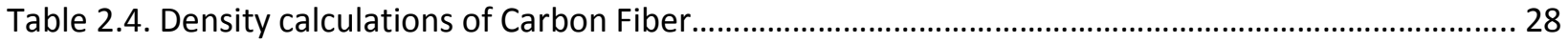

Table 2.5. Weight fiber fractions calculated experimentally and theoretically........................................... 30

Table 2.6. Experimental mechanical properties of Hexcel HRH-10-1/3-5 honeycomb.............................. 32

Table 2.7. Effect of adhesive layer on the ultimate load at initial failure .................................................. 34

Table 3.1. Beam and Plate Theory midspan deflections for varying core angle specimens at $100 \mathrm{lbf} . . . . . . .50$

Table 3.2. Beam Theory midspan deflections for varying Face sheet thickness specimens at $100 \mathrm{lbf} . . . . . .51$

Table 3.3. Plate Theory midspan deflections for varying Face sheet thickness specimens at $100 \mathrm{lbf} . . . . . . .51$

Table 3.4. Differences between Beam and Plate Theory midspan deflections for varying Face sheet

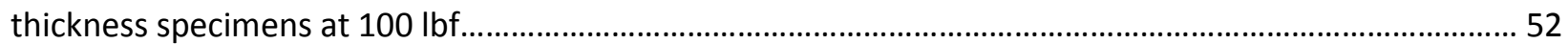

Table 4.1. Effects of core angle on composite sandwich structural properties............................................ 57

Table 4.2. Theoretical and Experimental mid-span deflection comparison with respect to Core Ribbon

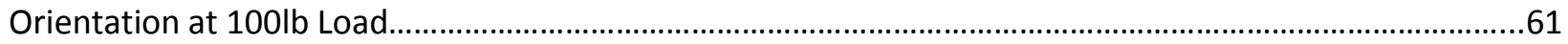

Table 4.3. Experimental Failure Loads for varying Face sheet thickness specimens (lbf)........................... 62

Table 4.4. Experimental Midspan Failure Extensions varying Face sheet thickness (in)............................. 65

Table 5.1. Mechanical and part properties of 6367 A280-5H used in ABAQUS Model............................... 69

Table 5.2. Mechanical and part properties of HRH-10-1/8-5 Honeycomb used in ABAQUS Model............ 70

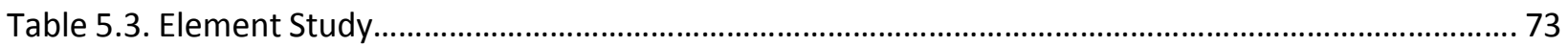

Table 5.4. Mechanical and part properties used for FEA model validation................................................. 75

Table 5.5. Comparison of Theoretical and Experimental deflection for model validation with

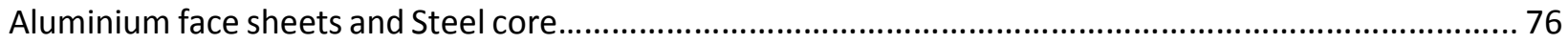

Table 6.1. Comparison of FEA, Experimental and Theoretical data for Core Ribbon Orientation

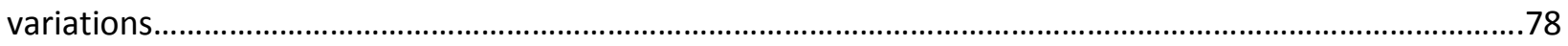

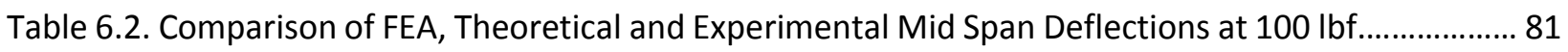

Table 6.3. Difference Between Finite Element and Theoretical Midspan Deflection for Varying Face

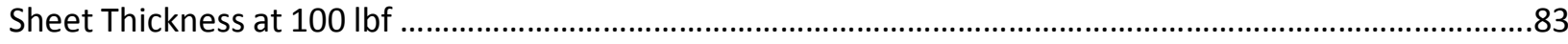

Table 6.4. Difference Between Finite Element and Experimental Midspan Deflection for Varying Face

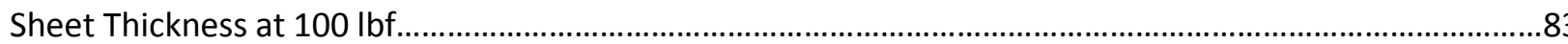


Over 50\% composite commercial plane - Boeing's 787 Dreamliner. 20

\section{LIST OF FIGURES}

Figure 1.1. Composite Baseball bat (left) and Boeing 777 (right) are examples of recent technology using various composites ${ }^{1}$...

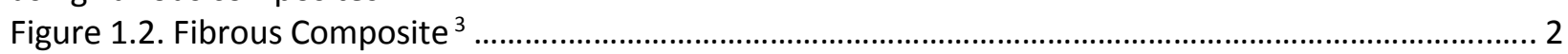

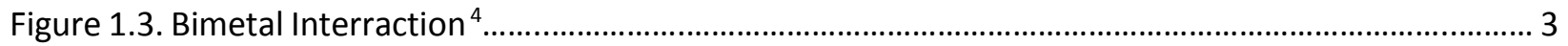

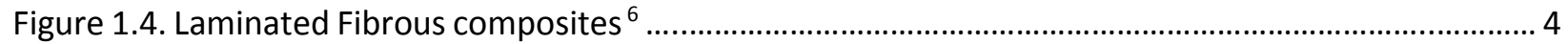

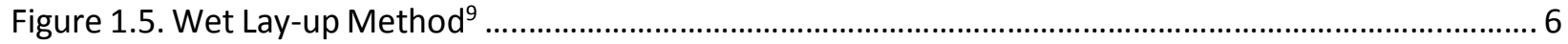

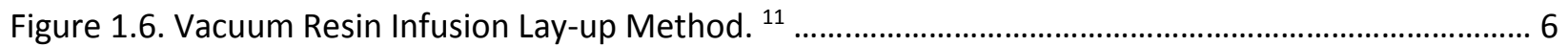

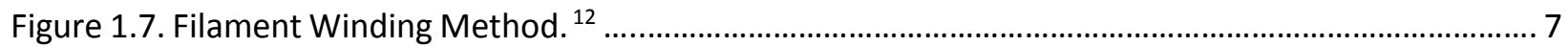

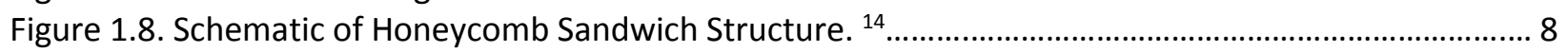

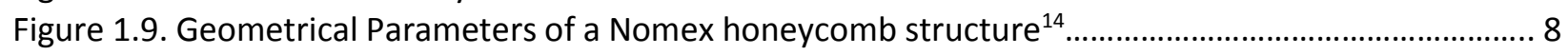

Figure 1.10. Sandwich panels with (a) corrugated (b) foam and (c) honeycomb core ${ }^{19} \ldots \ldots \ldots \ldots \ldots \ldots \ldots \ldots \ldots . . . . . . . . . . .10$

Figure 1.11. Over 50\% composite commercial plane - Boeing's 787 Dreamliner. ${ }^{20} \ldots \ldots \ldots \ldots \ldots \ldots \ldots . . . . . . . . . .11$

Figure 1.12. Schematic of Facesheet/Honeycomb Interface. ${ }^{24}$................................................................ 13

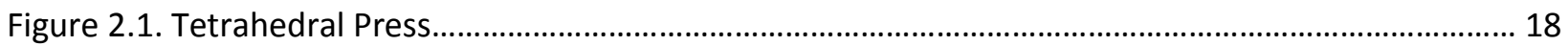

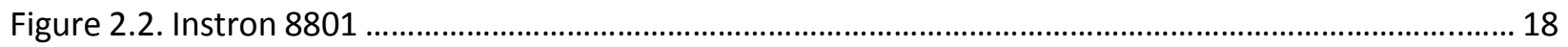

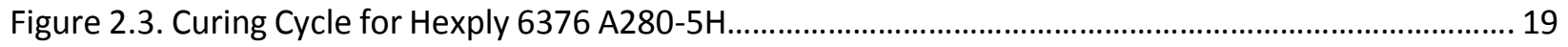

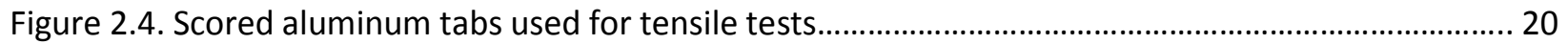

Figure 2.5. 1614 A\&B Structural adhesive from 3M used on tensile testing tabs........................................ 21

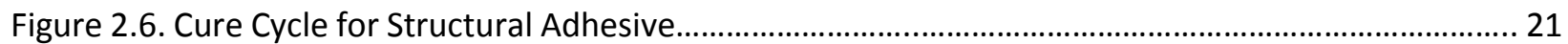

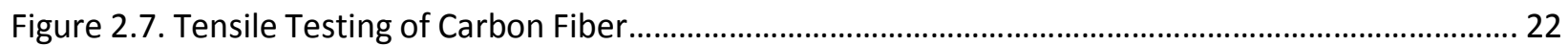

Figure 2.8. Samples failing at or near grip fixture are discarded .............................................................. 22

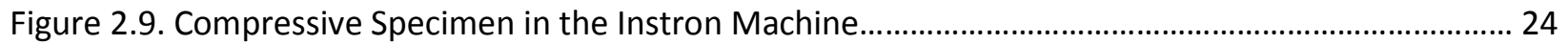

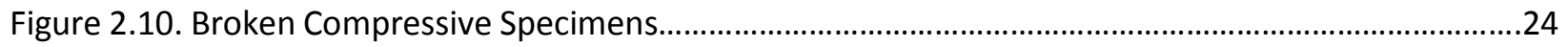

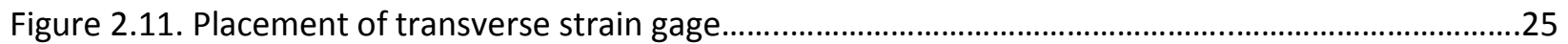

Figure 2.12. Strain versus load in longitudinal and transverse orientations of the carbon fiber..................26

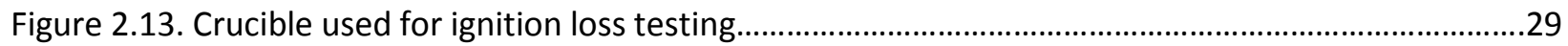

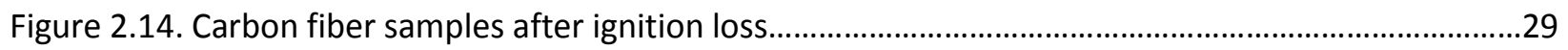

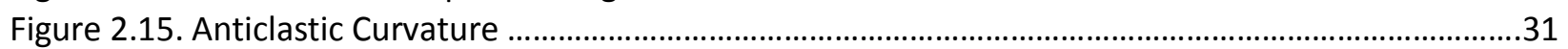

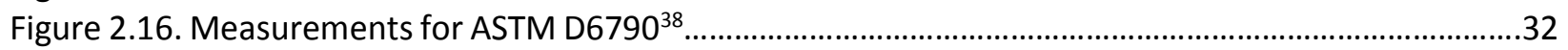

Figure 2.17. Viscid side of adhesive placed against honeycomb core.......................................................33

Figure 2.18. Load versus Extension plot for 3 ply Sandwich structures with and without adhesive layers..34

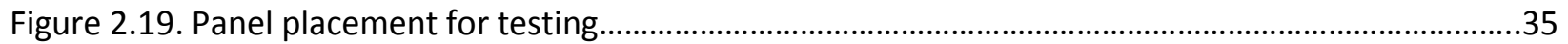

Figure 3.1. Core angle vs. yielding loads for three main types of failure ................................................. 46

Figure 3.2. Failure Loads for Specimens with Varying Top Face Sheet thickness and Bottom Layer

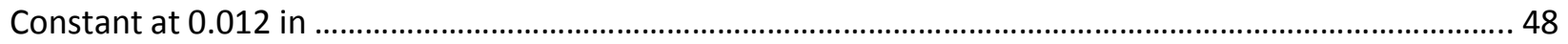

Figure 3.3. Static failure mode map for 0 degree core angle .................................................................. 49

Figure 3.4. Normalized strength to weight ratio with respect to top and bottom face sheet thickness..... 55

Figure 4.1. Sandwich material with Hookean Response region................................................................. 56

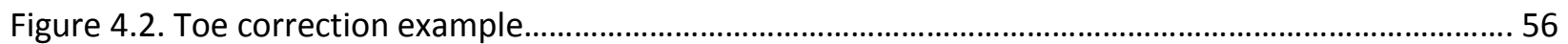

Figure 4.3. Failure Load and Extension vs core orientation..................................................................... 58

Figure 4.4. Theoretical maximum facing stress at failure vs. core orientation ............................................6 60

Figure 4.5. Normalised Stiffness and Strength with respect to to core ribbon orientation .........................6 60

Figure 4.6. Failure Load for varying face sheet thickness, Constant top layer.............................................63

Figure 4.7. Failure Load for Varying Face Sheet Thickness, Constant Bottom Layer .................................. 64 
Figure 4.8. Three Dimensional Perspective of Failure Loads for varying Face Sheet Thickness's.

Figure 4.9. Three Dimensional Perspective of Failure Extensions for varying Face Sheet Thickness's ...... 66

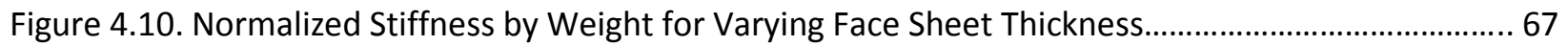

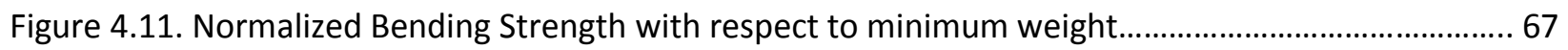

Figure 5.1. FEA Tie constraints on top and bottom shell elements .............................................................70

Figure 5.2. FEA Boundary Conditions and Loads....................................................................................71

Figure 5.3. Convergence Plot for Midspan Beam deflection modelled in Abaqus........................................ 76

Figure 6.1. Comparison of Experimental, FEA, and Theoretical deflection for Varying Core Angle............ 79

Figure 6.2. Stress distribution for Beam for Core orientation of 0 degrees at $100 \mathrm{lbf}$ applied load ............80

Figure 6.3. Deflection for Beam with Core orientation of 0 degrees at $100 \mathrm{lbf}$ applied load ........................80

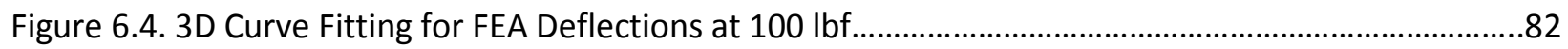

Figure 6.5. FEA, Experimental and Analytical Comparison for all configurations with 0.012in

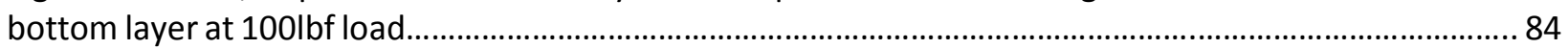

Figure 6.6. FEA, Experimental and Analytical Comparison for all configurations with 0.012in bottom

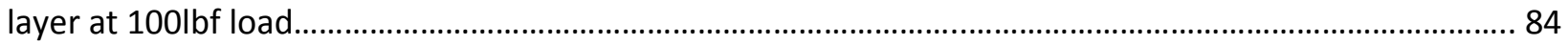

Figure 6.7. FEA, Experimental and Analytical Comparison for all configurations with 0.024in

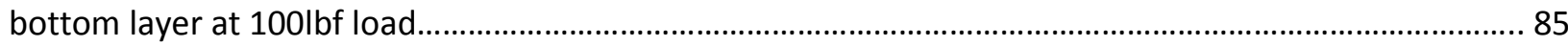

Figure 6.8. FEA, Experimental and Analytical Comparison for all configurations with 0.048in

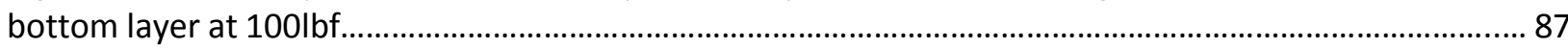

Figure 6.9. FEA, Experimental and Analytical Comparison for all configurations with 0.06in bottom layer at $100 \mathrm{lbf}$ load. 


\section{LIST OF MATLAB CODE}

A.8.1. Classical Beam and Plate Theory for Midspan Deflections of Specimens with Varying Core

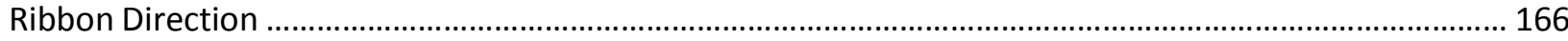

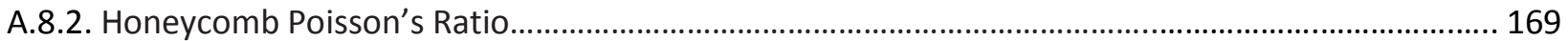

A.8.3. Experimental Density Measurements for Composite Laminates....................................................... 170

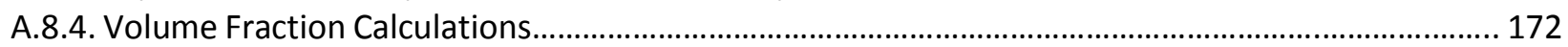

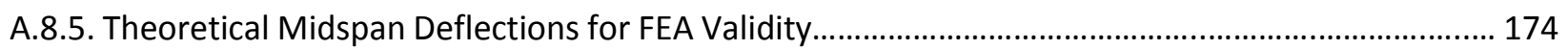

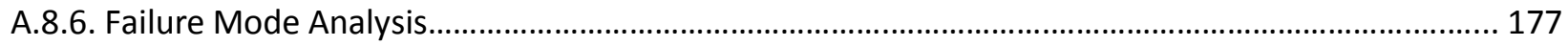

A.8.7. Master Code for all varying face thickness Matlab files.................................................................. 186

A.8.8. Data Manipulation Code for Varying Face Sheet Thickness Specimens........................................... 188

A.8.9. Plots Experimental Graphs for Varying Face Sheet Thickness's ...................................................... 193

A.8.10. Comparison of FEA, Theoretical and Experimental Deflections at 100lbf...................................... 204

A.8.11. Minimum and Maximum Core weight for Optimum Strength with respect to minimum

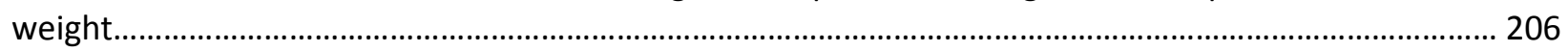

A.8.12. Theoretical Beam and Plate Theory values for varying facing thickness...................................... 211

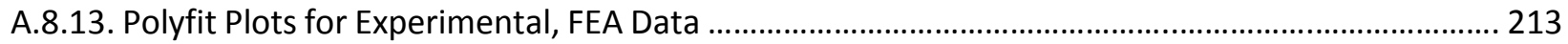

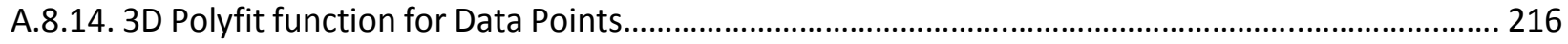

A.8.15. Theoretical Maximum Bending Strength With Respect to Minimum Weight .............................. 223 


\section{Nomenclature}

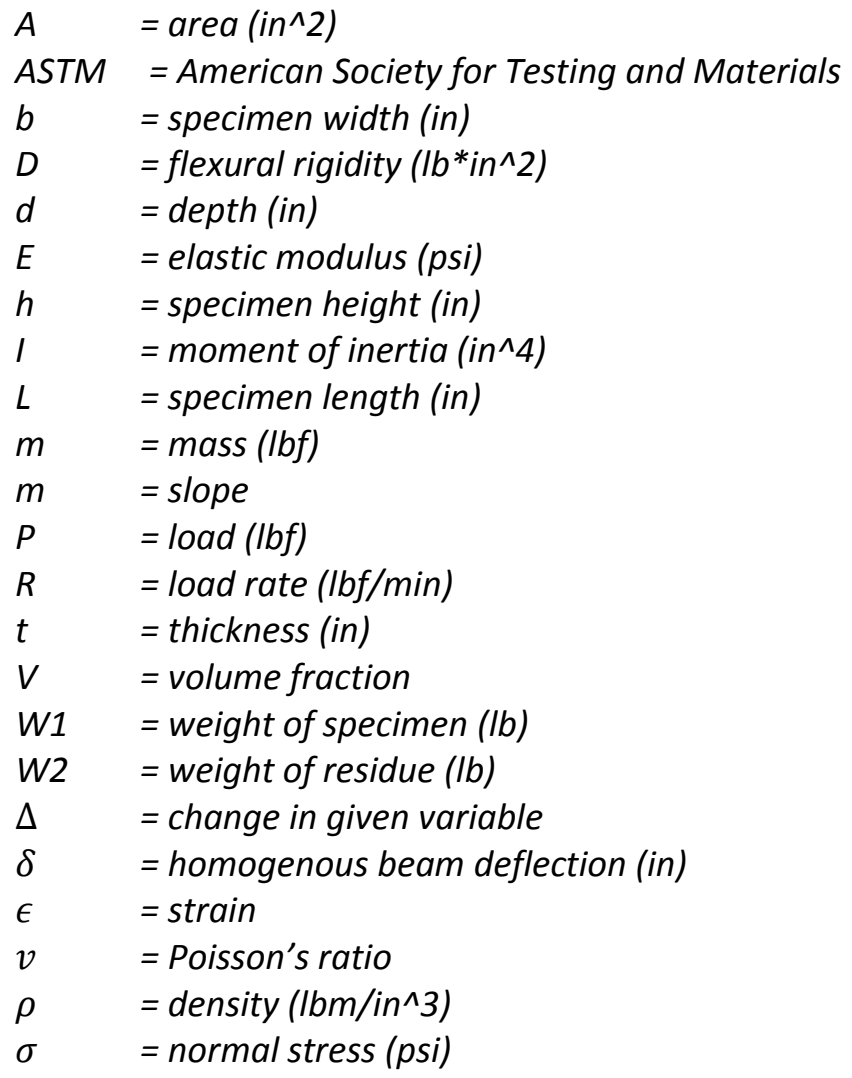

\section{Subscripts}

C = Compression

hc = HoneycombCore

fibers = relating to the fibers

fs $\quad=$ face sheets

$L \quad=$ Longitudinal

Matrix = relating to the matrix

Max = maximum

Min = minimum

$T=$ Transverse

$Y=y$-direction

$X \quad=x$-direction 


\subsection{Introduction}

\subsection{Introduction to Composites}

Composites have been integrated into just about every field of today's engineering world. As technology advances, so too has the demand for lighter materials with an increase in performance capabilities. Composites have come a long way from mud and straw used by our ancestors to the integration into advanced systems including the aerospace and sporting industries shown in Figure 1.1.

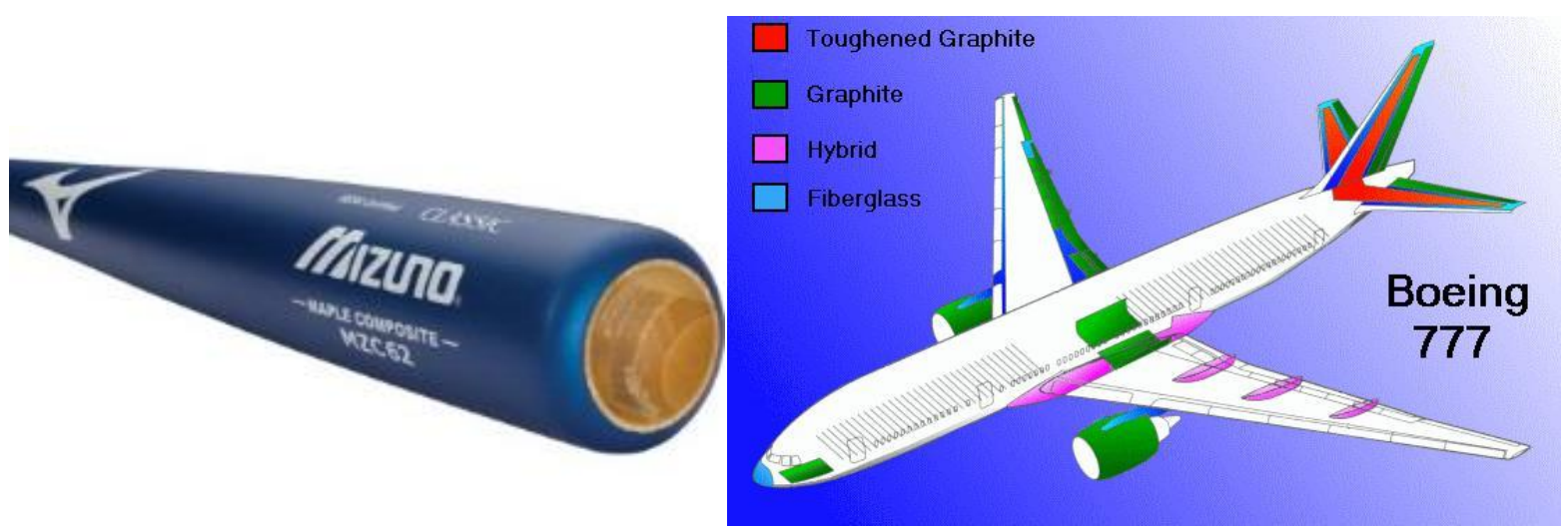

Figure 1.1. Composite Baseball bat (left) and Boeing 777 (right) are examples of recent technology using various composites ${ }^{1}$

A composite material consists of two or more materials, with an interface between them. The composite consists of strong fibrous materials surrounded by a weaker matrix material, which distributes the load to the fibers. Principal fiber materials include but not limited to, aramid or Kevlar (very light), glass, Carbon or Boron (high strength), and Silicon carbide (high temperature resistance). Most matrix materials include but are not limited to; Polymeric matrix's (thermoplastic and thermoset resins), Mineral matrix's (silicon carbide, carbon), and metallic matrix's (aluminum alloys, titanium alloys, and orientated eutectics). ${ }^{2}$

Composites are used because of their structural properties and their electrical, thermal, and environmental applications. Creating a composite does not increase all mechanical properties of its

${ }^{1}$ Graduate, Aerospace Engineering, 1 Grand Avenue San Luis Obispo, California 93407 
constituent materials; however, a composite can be optimized to meet the property needs of an application. Composites emphasize those materials that contain a continuous matrix that binds together with an array of stronger reinforcing material. The integration of the two result in structural properties that are superior to its' make up materials. The improved structural properties generally result from a load-sharing mechanism. Composites typically have a fiber that is stiff and strong whilst the matrix is more ductile. Many types of reinforcements also often have good thermal and electrical conductivity with a coefficient of thermal expansion (CTE) that is less than the matrix, and/or has good wear resistance. Composites allow for a weight reduction of $10-50 \%$ with equal or greater performance with a $10-20 \%$ decrease in material and manufacturing cost when compared with the same piece of the conventional metal material previously used. In an aircraft the saving in mass of using composites in turn leads to reduced fuel mass which results in increased payload mass or performance. Also composites have a good fatigue and corrosion resistance which leads to long term savings and a reduction in maintenance costs of the part. ${ }^{2}$ When the requirements for the application have been determined, the engineer can then decide on material choice, fiber and matrix combination, number of laminates and direction, and layup/curing technique needed to produce a composite that meets all design requirements.

\subsection{Types of Composites}

There are three types of composite materials: fibrous, laminated and particulate composites. Fibrous composites contain fibers that are held together by a matrix shown in Figure 1.2. Laminated composites consist of two or more layers of a material that are bonded together. Particulate composites are made up

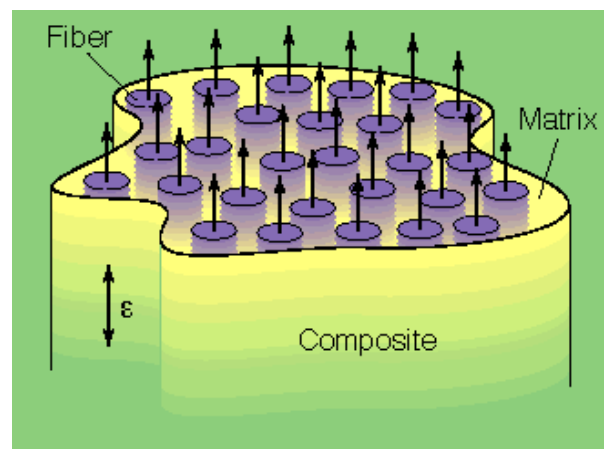

Figure 1.2. Fibrous Composite ${ }^{3}$ of fibrous particles with a matrix such as concrete or a cake. 
Laminated composites are created by layering different materials to create a new macroscopic structure that combines different aspects of the constituent materials. Laminated composites can be broken up into 5 categories: laminated fibrous composites, plastic based laminates, clad metals, laminated glass and bimetals. Bimetals, shown in Figure 1.3, are made up of two materials with different thermal expansion coefficients. When a significant temperature change is induced on the bimetal; the

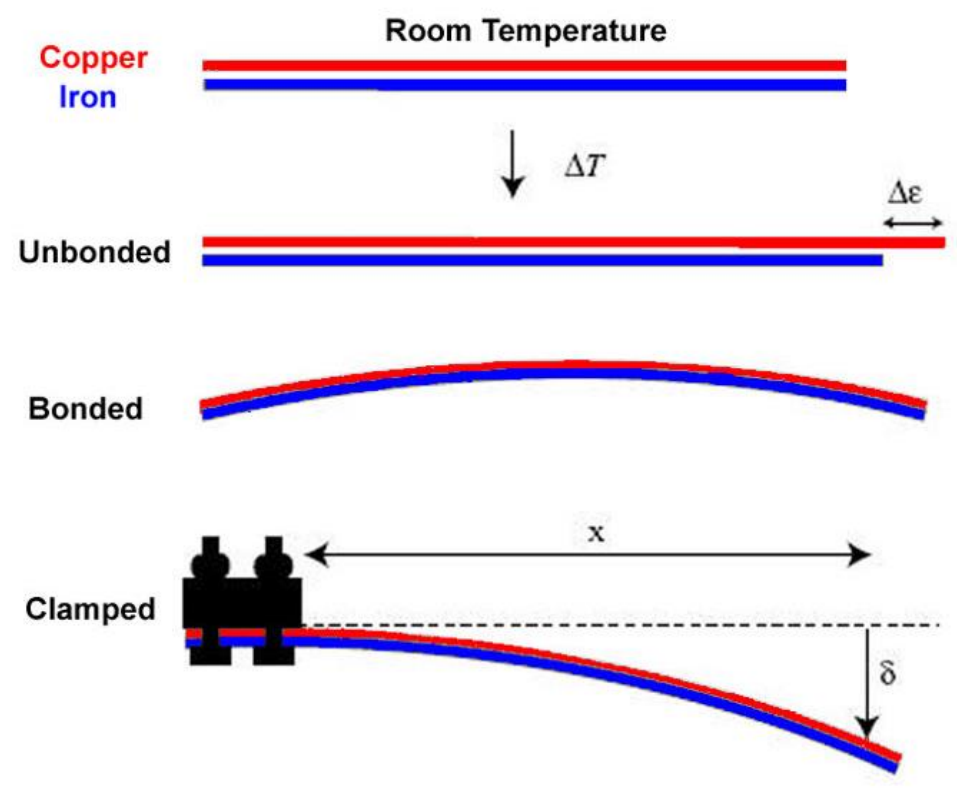

Figure 1.3. Bimetal Interaction ${ }^{4}$

change results in a deflection of the bimetal due to each metal expanding or retracting at different rates.

Clad metal composites is the bonding together of dissimilar metals to obtain the properties of the constitutent metals. An example is galvanizing metal parts to obtain non-corrosive qualities. Laminated glass can tolerate higher strains than traditional glass and often have scratch resistive qualities. An example of laminated glass is saftey glass.

Plastic based laminates are layers of different materials that are saturated with other materials and then treated for many purposes. An example of a plastic based laminate is Arborite, which consists 
of layers of phenolic resin impregnated paper overlaid by plastic saturated sheets, which is then overlaid with a plastic cellulose mat. The layers are then fused together with heat and pressure. ${ }^{5}$

Laminated fibrous composites are a mixture of laminated composites and fibrous composites. Each layer is composed of fibers and a matrix and then stacked or laminated with other layers in varied orientations so that the whole composite will have more or less stiffness in the required loading direction shown in Figure 1.4. This category is widely used in industry to meet specific design requirements.

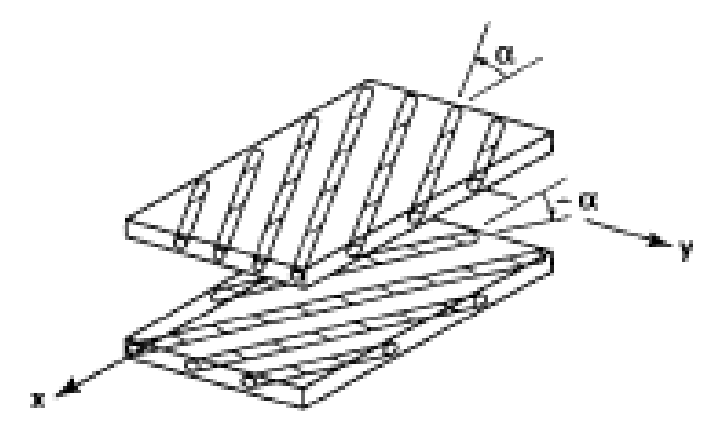

Figure 1.4. Laminated Fibrous composites ${ }^{6}$

The idea behind fibrous composites is fairly simple; a matrix that supports protects and distributes the load evenly to the fibers. The bonded materials produce improved mechanical properties over the properties of the materials by themselves. The key to the improved strength of the composite is because of the orientation and geometry of the fibers. By optimizing the fibers direction and by aligning the fibers with the load direction, the composite will be lighter whilst maintaining the needed strength. The matrix binds the fibers, giving protection from damage and corrosion whilst distributing the load between the fibers. The matrix often puts limitations on the composite, such as operational temperature ranges. Often polymers and plastics are used as composite matrices due to easy production, low cost, high availability, low specific gravity and high chemical resistance. The 
disadvantages of polymer matrices are that they have low moduli, strength and operational temperature ranges. Other materials can be used for matrices, including metal that provide higher moduli larger operable temperature ranges and high impact strength, but with higher density, subject to corrosion and higher fabrication cost. ${ }^{7}$

Fibrous composites have been used for applications in aircraft from the first flight of the Wright Brothers' Flyer I, in North Carolina on December 17, 1903, to the many applications now used in military and civil aircraft, satellites, launch vehicles and even automobiles and sports equipment. In the early days, aircraft structure was primarily composites made of wood, wire and fabric. It wasn't until the 1930 's that aluminum alloys became the predominant aircraft structure material, although wooden structures were still used until World War II. Recent military and civil aircraft have worked on replacing the secondary structure with fibrous composites made up of carbon, Kevlar, glass or a hybrid of the three. The reason behind the influx of fibrous composites in manufacturing of parts is due to their high specific stiffness and strength, as well as the ability to shape and manufacture the composite to produce a greater aerodynamic structure than its metal counterpart. ${ }^{8}$

Particulate composites tend to be much weaker and less stiff than continuous fibrous composites, but that is countered by the decrease in manufacturing and material costs. Particulate composites usually contain less reinforcement due to processing difficulties and brittleness. An example of particulate composites is asphalt or concrete. The stones and small pebbles act as the particulate and the cement acts as the matrix. This study does not involve particulate composites and thus will not be talked upon further.

\subsection{Methods of Composite Fabrication}

In creating polymeric composites, there are currently four different types of layups that are most commonly used in industry. The most favored method is known as "Pre-preg" layup, which is a composite that has had the resin pre-impregnated into the composite when it is initially created. The 
advantages include a consistent fiber-to-resin ratio, which allows for the strongest part by layer possible when cured. Disadvantages of pre-preg include high material costs, very specific curing cycles, expensive machines for curing (Autoclaves or heat presses), and storage at temperatures for the pre-preg close to $0^{\circ} \mathrm{F}$ to insure the epoxy resin does not cure. ${ }^{10}$

The second method is known as the "wet-layup" as seen in Figure 1.5, which involves taking dry fibers and ingraining resin by hand, before curing. Advantages include low cost, simple process, no

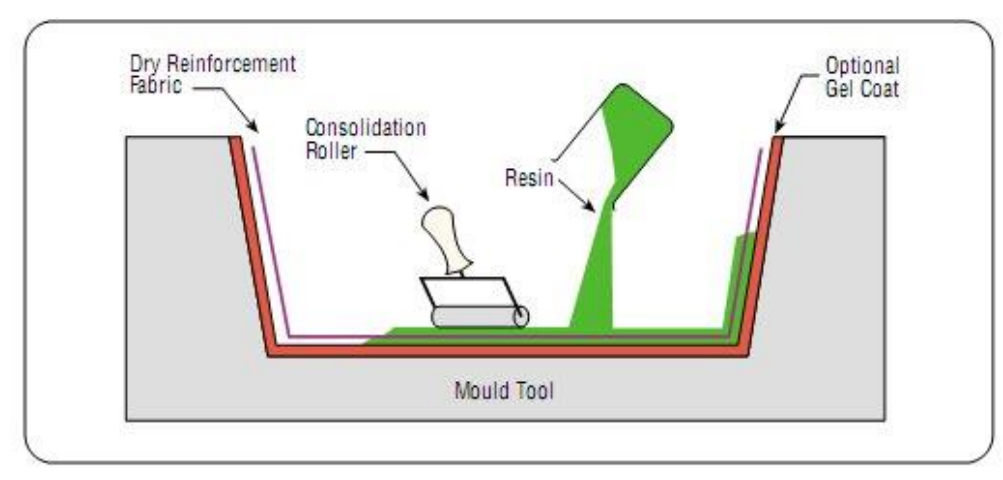

Figure 1.5. Wet Lay-up Method ${ }^{9}$

machines needed for curing, no stringent curing cycles. Disadvantages include inconsistencies in fiberto-resin ration, and damage to fibers can occur during resin integration. ${ }^{10}$

The third method is known as resin infusion or vacuum assisted transfer-molding system (VARTM), shown in Figure 1.6. This method uses a vacuum to pull resin across a composite part, whilst

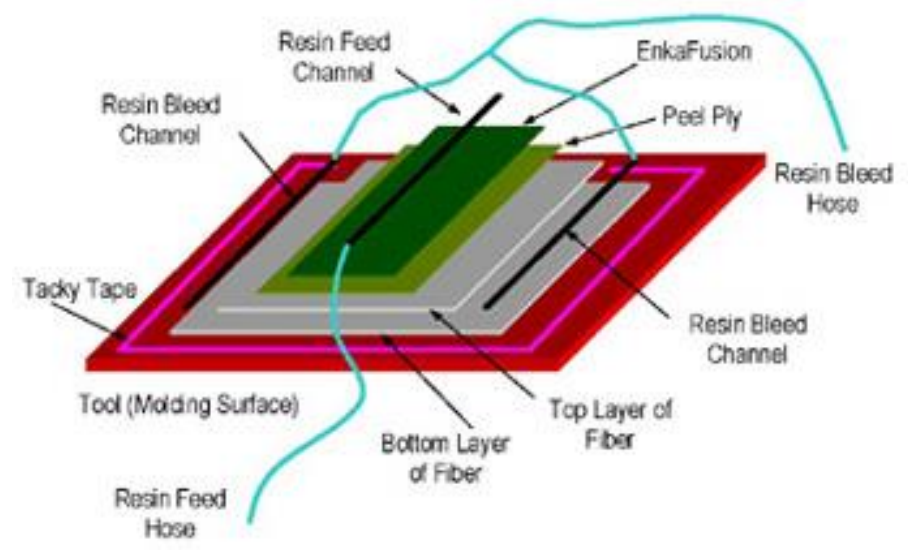

Figure 1.6. Vacuum Resin Infusion Lay-up Method ${ }^{11}$ 
also infusing the fibers with the resin. Advantages include a balance between quality and cost, potentially minimal damage to fibers due to any handling of the fibers, and fairly consistent fiber-toresin ratio. Disadvantages include limited working time reliant on resin setting rate and limited part size due to resin being sucked across part by vacuum. Big parts tend to prove troublesome with fiber-to resin ratio inconsistencies. This method is a happy medium between wet-layup and pre-preg; it's quick, not as costly as pre-preg and better fiber-to-resin ratios as wet-layup. ${ }^{10}$

The last main method is known as filament winding, where, as shown in Figure 1.7, fibers and resin are tension-wound together over a mold or mandrel and, after curing, the mold or mandrel is removed. This process is usually used for composite tubing, is relatively new and has had many improvements since its inception.

As the integration of composite materials, specifically in military aircraft, increased from the 1960's onward, improvements in fibers and matrix materials resulted in carbon fiber reinforced plastics (CFRP) composites. With improved mechanical properties, CFRP's replaced the more conventional

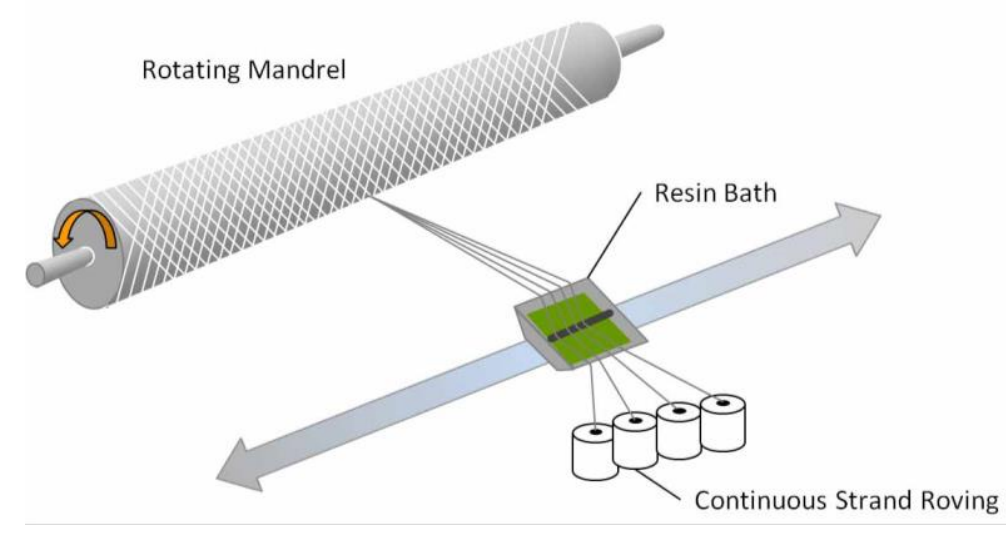

Figure 1.7. Filament Winding Method ${ }^{12}$

titanium alloys and aluminum for primary aircraft structures. The main advantages of CFRP include complex shape manufacture, improved fatigue life, design optimization, reduction in excess material, significant part mass reduction and improved corrosion resistance. The disadvantages include high material and processing costs, susceptibility to impact damage ${ }^{13}$, more advanced repairs and 
inspections, and size effects on strength. Innovation is paramount in making composites as affordable as, or as close to the affordability of, the conventional material counterparts.

\subsection{Composite Sandwich Panels}

In the aircraft industry every extra kilogram of structural mass taken off, means an increase in payload mass as well as a decrease in fuel mass, which trickles down to an increase in profit. Thus honeycomb sandwich panels were one of the outcomes of research into decreasing structural mass. Sandwich panel structures consist of two thin face sheets on the top and bottom of a shear resistant lightweight core as shown in Figure 1.8.

The relative separation of the stable face sheets result in high stiffness to weight ratios.

Essentially the honeycomb was used as a shear web between two upper and lower skins, with the early honeycomb sandwiches made of balsa wood as the core and plywood as the skins. With the

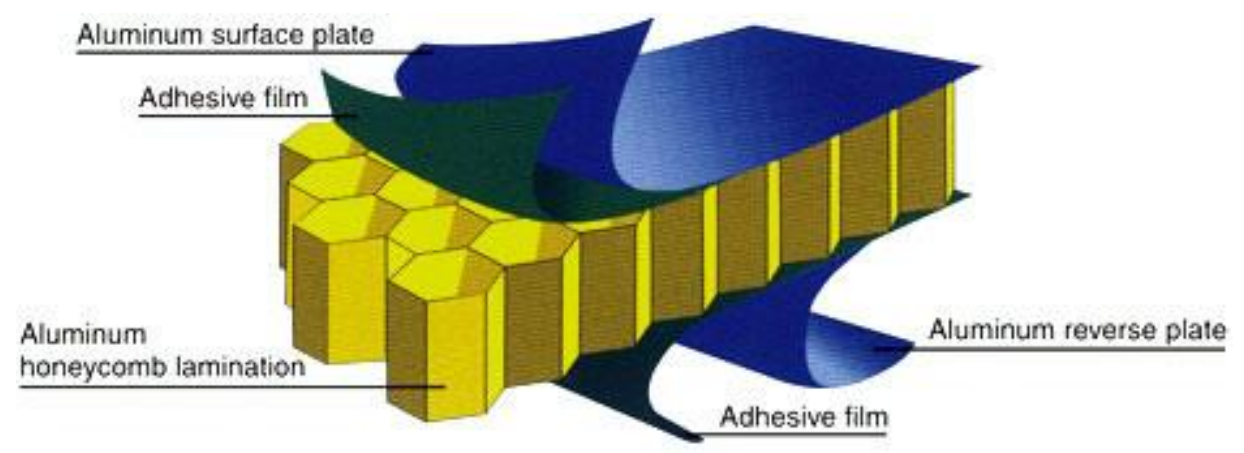

Figure 1.8. Schematic of Honeycomb Sandwich Structure ${ }^{14}$

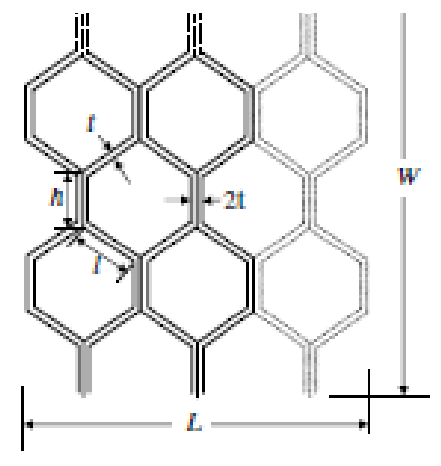

Figure 1.9. Geometrical Parameters of a Nomex honeycomb structure ${ }^{14}$ 
development of Epoxy resin, it was possible to bond aluminum skins to an aluminum honeycomb core, which occurred in $1954 .{ }^{15}$ Since then, many advancements have been made in honeycomb studies, with the most commonly used honeycomb for aircraft structures being Nomex Honeycomb. ${ }^{16}$ Figure 1.9 shows the honeycomb structure of Nomex, which is used extensively in this thesis. Nomex is constructed from ribbons of aramid fibers running in the longitudinal direction that are fused together with glue at sections along the ribbon. By pulling on the ribbons in the direction perpendicular to the longitudinal direction, the ribbons expand to form a honeycomb structure. The fiber structure is then dipped into phenolic resin to strengthen the walls of the honeycomb, which makes the honeycomb anisotropic with respect to out-of-plane shear stiffness and strength. Choo. F., et al. observed that honeycomb structures under tensile tests produced higher ultimate loads when the load was applied in the longitudinal (fiber) direction than in the direction perpendicular to the longitudinal direction. ${ }^{17}$

\subsubsection{Advantages and Disadvantages of Sandwich Composites}

Sandwich structures utilize each of its constituent materials' properties. The thin face sheets' high stiffness combined with low-density cores give a sandwich structure of high stiffness to weight ratio when compared with a face sheet beam of same weight, and a high bending strength to weight ratio. In addition to the efficiency between stiffness and strength, honeycomb sandwich panels are fairly fatigue resistant, great insulators or radiators depending on the core material selection, highly serviceable and have smooth aesthetically pleasing surfaces. ${ }^{18}$ Honeycomb sandwich panels are analogous to beams or plates. The use of honeycomb prevents buckling of the thin skins by providing the amount of shear strength to do so.

Honeycomb panels are lightweight, easy to work with, and not labor intensive. By increasing the thickness of the core, the composite panel's strength and flexural stiffness increases much like increasing the height of a beam, but without the weight increase shown in Table $1 .{ }^{19}$ This is due to an increase in the panels' moment of inertia. Composite panels are designed such that failure occurs in the core of the panel, thus shear strength is the main factor in design, which is the core's predominant material property. Composite panels are designed to meet the application requirements. They have the same normal strengths that composites 
have, due to the face sheets being constructed from materials of high modulus of elasticity's (when compared with the core) like fiber-resin mixtures, metal alloys and plastics. The cores have low elastic moduli that yield without failure in the high deflection regimes. Cores usually consist of metallic and fibrous honeycomb structures to opened and closed cell structured foams seen in Figure $1.10{ }^{19}$

Table 1.1. An example of sandwich panel structural efficiency with respect to weight ${ }^{19}$

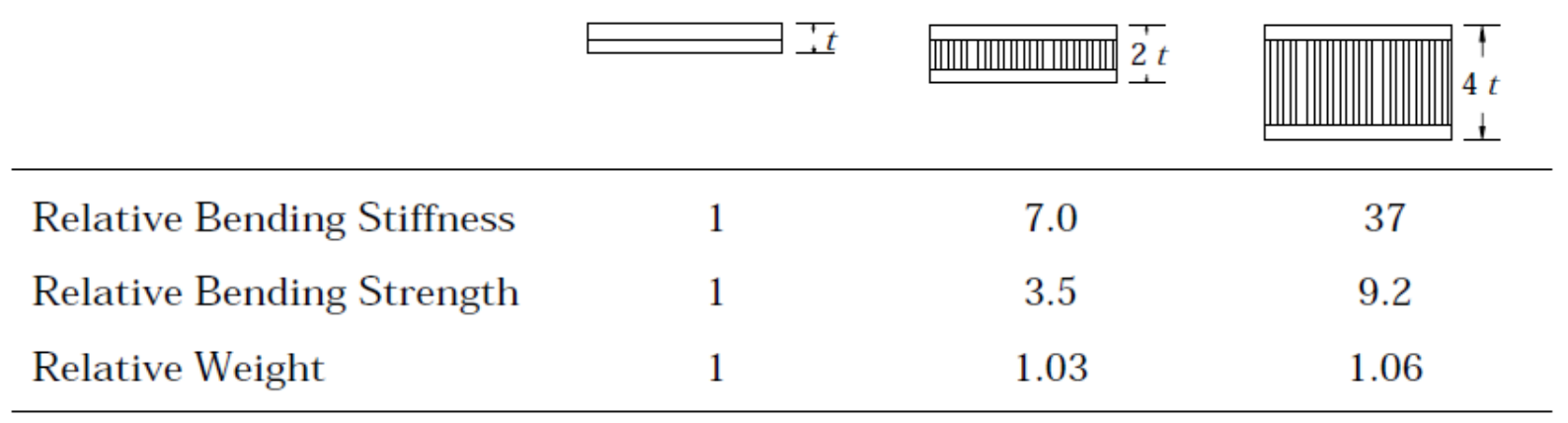

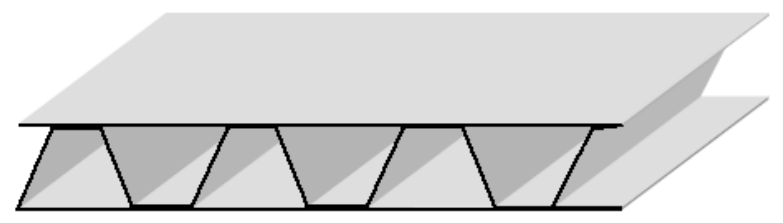

(a)

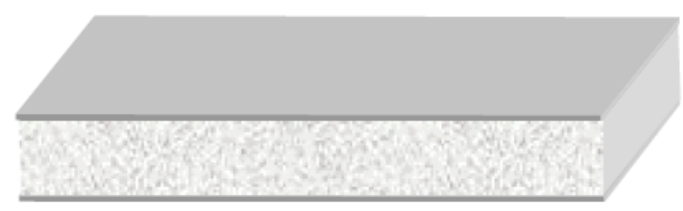

(b)

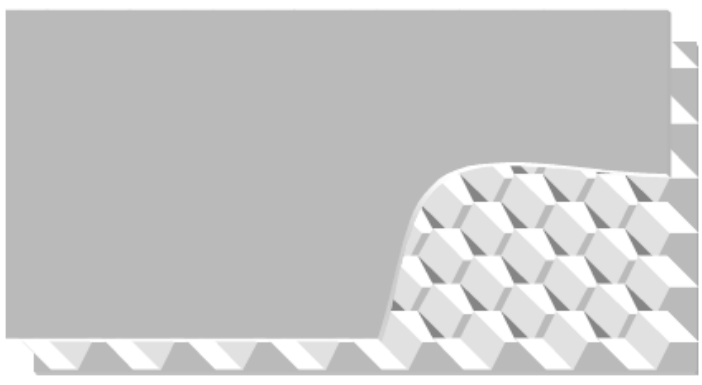

(c)

Figure 1.10. Sandwich panels with (a) corrugated (b) foam and (c) honeycomb core ${ }^{19}$ 
There are many standards, manufacturing techniques and accepted methods for constructing and testing materials such as metals. As sandwich composite structures are relatively new, there are not nearly as many standards for manufacturing and testing, particularly with the inclusion of honeycomb. Quality control thus is difficult to ensure correct integration into the strict design requirements of the aerospace industry. This results in a much higher safety factor when constructing the sandwich design, which is counterproductive to the main goal of reducing weight.

\subsubsection{Aerospace Industrial Application}

The application of composites is in high demand due to their favorable mechanical characteristics and material properties to current materials used, especially in the aerospace industry. Composite sandwich structures have revolutionized the aerospace industry because of their high stiffness and lightweight attributes when compared with aluminum, the aviation standard. Sandwich structures have proven particular advancements in the latest spacecraft, automobiles, airplanes and racing yachts to name a few. In the civil industry, sandwich composites have revolutionized bridge and flooring structures. In the auto industry, companies have shifted to the use of fiberglass and carbon fiber

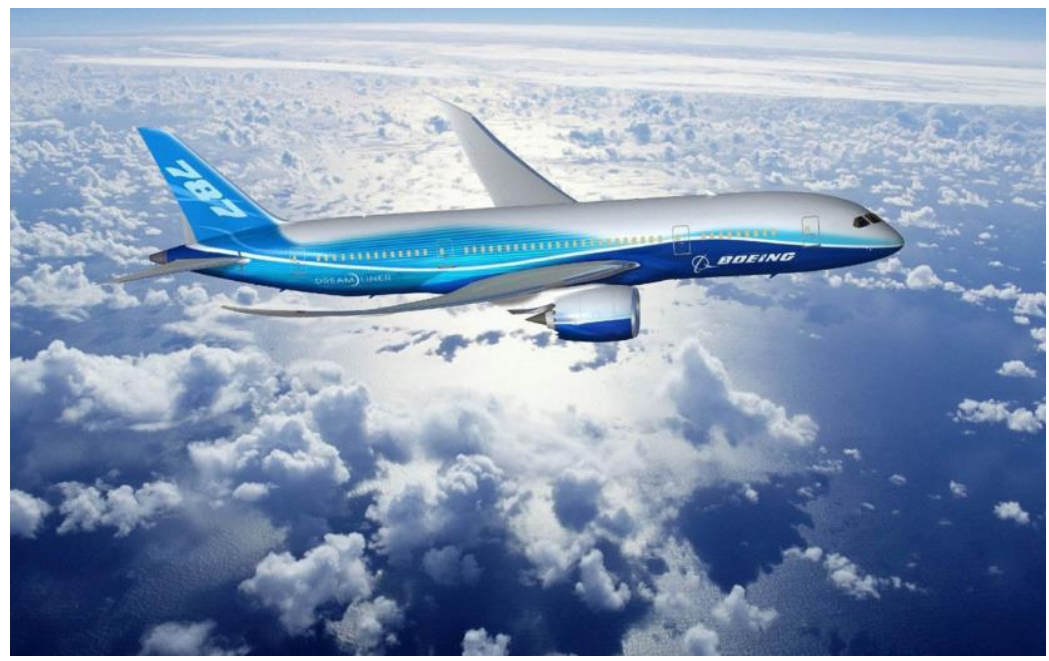

Figure 1.11. Over 50\% composite commercial plane - Boeing's 787 Dreamliner. ${ }^{20}$ 
to dramatically decrease weight, and thus directly increase performance. These advancements are accounted mostly to the large weight reduction sandwich structures and composites offer over traditional materials. The aerospace and military industry has had the most dramatic advancements due to the use of sandwich composites. Aircraft performance, for the most part is directly affected by weight. Sandwich structures can be almost as stiff as steel whilst the low core density maintains the sandwich structure weight at a fraction of that compared with a comparable steel beam. Sandwich structures can be integrated into such aircraft parts as the wings, floor, ceiling, fuselage and cargo compartment paneling, and even control surfaces. Figure 1.11 shows the Boeing 787: the most recent aircraft to be constructed out of mostly composite materials, allowing for a $20 \%$ increase in fuel efficiency and $40 \%$ increase in engine efficiency over its' replacement, the Boeing $767 .^{21}$

\subsection{Previous Work}

Reviewing previous works is vital in preventing time wasted by conducting redundant work. Reviewing past materials can help the researcher see what methods have been successes and failures, which prove invaluable to producing progressive works. All the previous works reviewed in this thesis have been published and peer reviewed. The most helpful works have been Frostig et al. Bending of Nonsymmetrical Sandwich Beams with Transversely Flexible Core ${ }^{22}$, Lim, T.S., Lee, C.S. and Lee. D.G. Failure Modes of Foam Core Sandwich Beams under Static and Impact Loads ${ }^{23}$, Foo. C., Chai. G., Seah. L., Mechanical Properties of Nomex Material and Nomex Honeycomb Structure ${ }^{17}$, Whitney, J. A Local Model for Bending of Weak Core Sandwich Plates ${ }^{44}$, Khan, S. Bonding of Sandwich Structures - The Face sheet/Honeycomb Interface ${ }^{14}$ and Allen, H. G., Analysis and Design of Structural Sandwich Panels. ${ }^{25}$

The bending of unsymmetrical sandwich beams with flexible, foam or honeycomb cores has been investigated analytically by Frostig et $\mathrm{al}^{22}$. Beams consisting of different upper and lower skins, with a soft core were tested, analyzing the effects of the flexibility of the core in the vertical direction, the bending behavior of the face sheets, and the overall panel behavior. Fibrous composite, and metallic 
face sheets have, as a rule of thumb approximately twice the tensile strength as compressive strength. By having a thicker face sheet at load application, it can prove beneficial to overall panel performance ${ }^{22}$. Khan's paper discusses that the most occurring failure of composite sandwich panels occurs at the de-bonding of the composite face sheets from the core, which is usually initiated by impact damage on the surface, which is then propagated by flexural load on the damaged portion subjected to cyclic fatigue or compression. Due to a difference in moduli between the face sheets and the honeycomb core, if a bending load is applied, a large shear stress generates at the interface. If a flaw is present at the face sheet-core interface, this shear stress can separate the face sheet from the honeycomb core. The quality of the adhesion between face sheet and core determines how much force a sandwich structure can hold and so it is crucial that the quality of this bond is a major design factor of honeycomb sandwich panels. ${ }^{24}$

There are two methods to bonding the composite face sheets and the honeycomb. The first, an adhesive film is placed on the bottom and top faces of the honeycomb, where the cured or uncured face sheets are placed. The whole piece is then cured in an autoclave. This process melts the adhesive resin, which creates a bond between the composite face sheet and the honeycomb wall. The second method has uncured pre-preg face sheets placed on the top and bottom surfaces of the honeycomb and the whole panel is cured in an autoclave, whereupon the resin from the pre-preg melts and creates a bond between face sheets and honeycomb walls. An optimal bond is formed between face sheet and honeycomb wall when the resin flows to form a symmetric fillet on the honeycomb wall as seen in Figure 1.12. The edge of the honeycomb wall should be

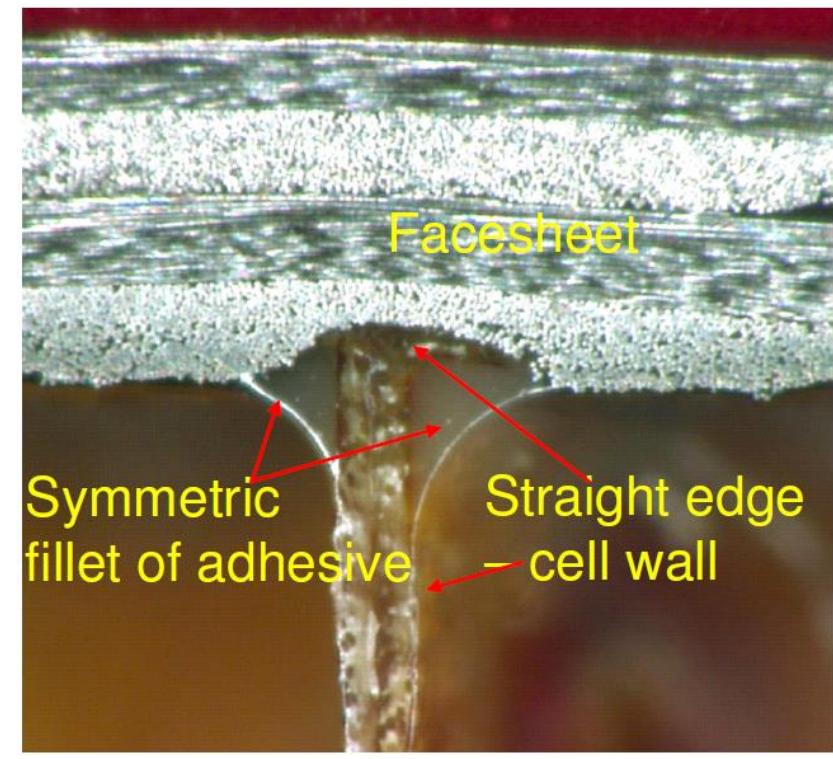

Figure 1.12. Schematic of facesheet/Honeycomb Interface. $^{24}$ 
perpendicular to face sheet and should indent the face sheet marginally to make an ideal interface bond.

During the bonding process, the honeycomb does not melt or flow and thus the surface quality of the honeycomb plays a role in bond quality. It is paramount that the honeycomb-bonding surface is clean of fibers so that delamination failure will not occur as a result of damaged fibers. A clean surface can be achieved through wet sanding. ${ }^{24}$

Lim, Lee and Lee ${ }^{23}$ investigated the static failure modes of foam core sandwich panels experimentally using the static three point bending test with respect to face sheet thickness, span and core density. Triantafillou and Gibson ${ }^{26}$ developed failure equations to describe the failure load for face yielding, face wrinkling and core shear from which the failure mode map was constructed. Analytical failure loads and modes were obtained from the theory of sandwich beams on elastic foundation. The results were compared and showed good agreement with each other. Three transition equations were made using non-dimensional parameters, including strength ratio, moduli ratio, and normalized thickness and span. Using the transition equations, the general static failure mode map could be developed considering local deflection. ${ }^{23}$ There has been much analysis of panels, beams and struts of sandwich panels summarized by Allen ${ }^{25}$ and Plantema ${ }^{27}$. Allen ${ }^{25}$ modeled sandwich panels as a beam, with the assumptions that the core is homogenous, of much lower stiffness when compared with the face sheet material and the skin is relatively thin when compared with the core and later Ashby and Gibson $^{28}$ further developed this model. Optimization of skin and core thickness that satisfy stiffness constraints at minimum weight have been developed by Gibson and Triantafillou ${ }^{29}$. Some buckling and wrinkling behavior of sandwich panels under in-plane compression has been conducted by Kwon and Pearce ${ }^{45}$. 


\subsection{Main Objective and Scope of Research}

The main objective of this study is to investigate the effects of varying the Honeycomb core orientation on flexural characteristics of composite sandwich structures with constant face sheet thickness. Once the optimal core orientation is determined the objective is to investigate the effects of varying top and bottom face sheet ply count (thickness) with a constant honeycomb Nomex core. Typically, symmetry is used in composite sandwich design to account for multiple loading conditions. For example, an aircraft wing experiences torsion, compression and tension from its environment during flight. If the wing were simplified to a single composite sandwich, it can be seen that symmetry in construction is necessary as the loading direction could be in compression, tension or torsion at any given point. Composite sandwich structures in other sections of aircraft experience loading in only one direction for example, floor paneling for the most part, experience only static loading in one direction, and knowing that fibrous composites can take, depending on material selection, twice as much load in tension as compression, by adding extra layers to the side of the sandwich under the loading force, and/or subtracting layers on the opposite side, we can obtain two things: either increase performance in bending stiffness and/or strength whilst keeping weight constant or in the case of aerospace design; decreasing weight whilst maintaining or increasing performance.

Chapter 1 goes over the introduction of composite materials, from the varying composite types to different fabrication methods. The background and advantages of composite sandwich panels are discussed as well as their aerospace industrial applications. Previous honeycomb sandwich works are discussed and the main objective and scope of this research is explained. Chapter 2 outlines the manufacturing, procedure and experimental set-up for obtaining the mechanical properties of the composite sandwich structure's constituent materials and the sandwich structure as a whole using available ASTM standards. Chapter 3 entails the analytical analysis conducted using classical beam and plate theory for sandwiches with varying core ribbon orientation and varying face sheet thickness. A 
study into the necessity of adding an adhesive layer between carbon fiber pre-impregnated face sheets and Nomex honeycomb core is also conducted. Failure mode analysis for panels with equal face sheet thicknesses is conducted using carbon fiber and Nomex honeycomb with respect to core ribbon orientation. Optimization of bending stiffness and strength with respect to minimum weight is looked at. Chapter 4 discusses and analyzes the experimental data of specimens with varying core orientation and varying face sheet thickness. A Finite element model is developed in chapter 5 and FEA analysis is compared with experimental and analytical results and obtains good agreement with both. Chapter 6 concludes the study. 


\subsection{Manufacturing, Procedure and Experimental set-up}

This section covers the manufacturing and testing procedures for obtaining the mechanical properties of the carbon fiber face sheets, honeycomb core and sandwich structure as a whole. Composite materials have few ASTM standards to guide the tester with a proven method. There are some general guidelines that must be followed when testing composite specimens, however. It is paramount that extra precautions must be taken to prevent notches, uneven surfaces, and delamination when machining specimens. Caution must be taken when cutting sandwich panels to ensure the core does net get compromised due to low heat resistance. The testing of all specimen mechanical properties followed their respective ASTM standards shown in Table 2.1 below.

Table 2.1. ASTM Standards used in this experiment

\begin{tabular}{|c|l|l|}
\hline \multicolumn{1}{|c|}{ Material } & \multicolumn{1}{|c|}{ Mechanical Property } & \multicolumn{1}{c|}{ ASTM Standard } \\
\hline \multirow{4}{*}{ Carbon Fiber } & $\begin{array}{l}\text { Tensile/Compressive } \\
\text { stress/strength }\end{array}$ & D3039/D3410 \\
\cline { 2 - 3 } & $\begin{array}{l}\text { Tensile/Compressive Modulus of } \\
\text { Elasticity }\end{array}$ & D3039/D3410 \\
\cline { 2 - 3 } & Volume Fraction & D2584 \\
\cline { 2 - 3 } & Poisson's Ratio & E132 \\
\hline \multirow{4}{*}{ Honeycomb } & Poisson's Ratio & D6790 \\
\cline { 2 - 3 } & Core Shear Ultimate strength & C0393 \\
\cline { 2 - 3 } & Core Shear Modulus & D7250 \\
\hline \multirow{4}{*}{ Sandwich structure } & Flexural stiffness & D7250 \\
\cline { 2 - 3 } & Shear Rigidity & D7250 \\
\cline { 2 - 3 } & Facing Stress & \\
\hline
\end{tabular}


All specimens were cured in a tetrahedral press seen figure 2.1. All three-point bending tests were performed in an Instron 8801 universal test machine with a $100 \mathrm{kN}$ cell seen figure 2.2. Load and deflection were taken from the tests and results were obtained by applying the simplified beam theory. ${ }^{25}$

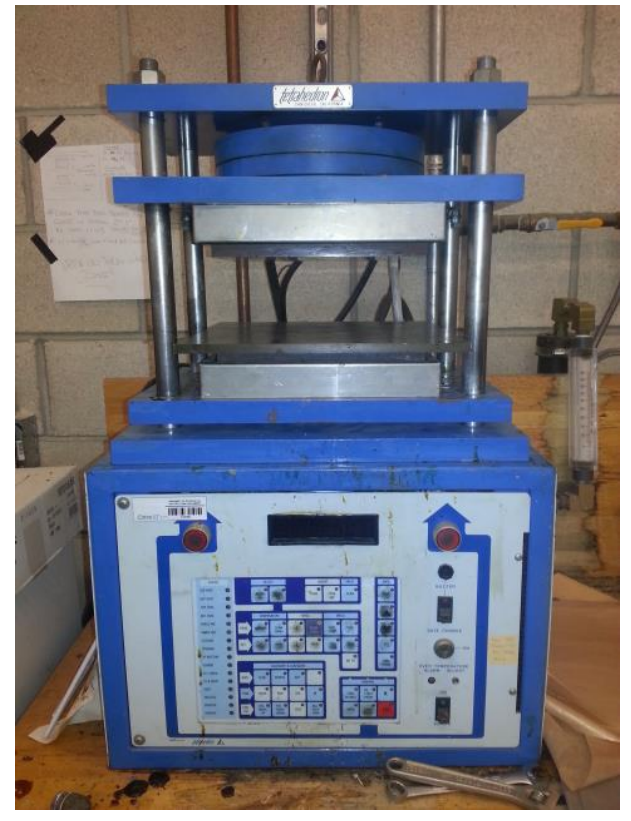

Figure 2.1. Tetrahedral Press

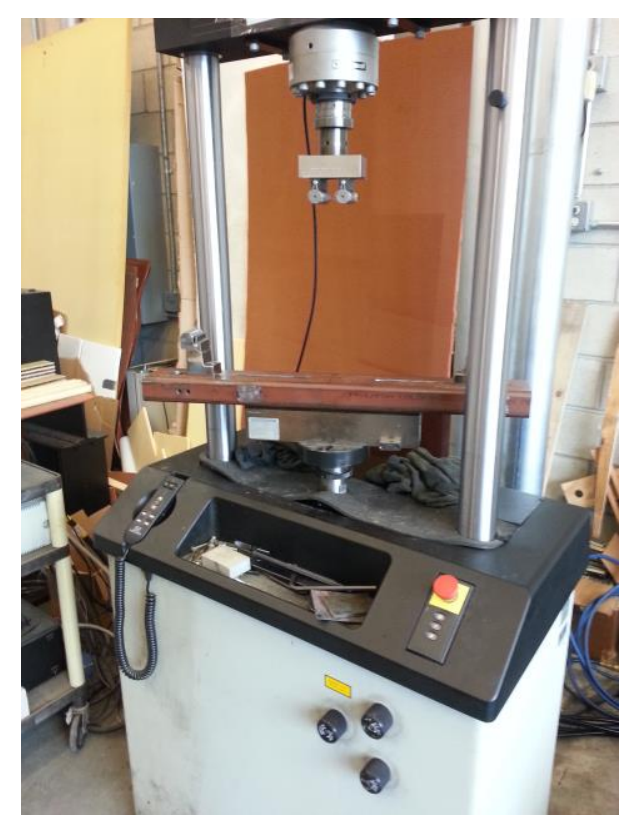

Figure 2.2. Instron 8801.

\subsection{Carbon Fiber Face Sheet Mechanical Properties}

The face sheet material used in the composite sandwich structures was Hexcel's pre-impregnated Hexply 6376 matrix with A280-5H bidirectional woven carbon fiber seen. There are several ways to cure pre-impregnated carbon fiber, but the chosen method was in a tetrahedron press, located in the Cal Poly Aerospace Structures/Composites Lab. 


\subsubsection{Tensile Mechanical Properties}

To determine the tensile properties of the face sheets of HexPly 6376 pre-preg, $12 \times 12$ inch plates were cut out and cured in a Tetrahedron heat press. HexPly 6376's curing cycle in a heat press can be seen below in Figure 2.2. The specimen is heated at a rate of $5 \%$ min up to $225^{\circ} \mathrm{F}$ and held at that temperature for 1 hour. The temperature is then ramped at $5 \%$ min up to $350^{\circ} \mathrm{F}$ and held at that temperature for 2 hours. The Temperature is then ramped down at a rate of $5 \% \mathrm{~min}$ down to $75^{\circ} \mathrm{F}$ and cycle is then terminated.

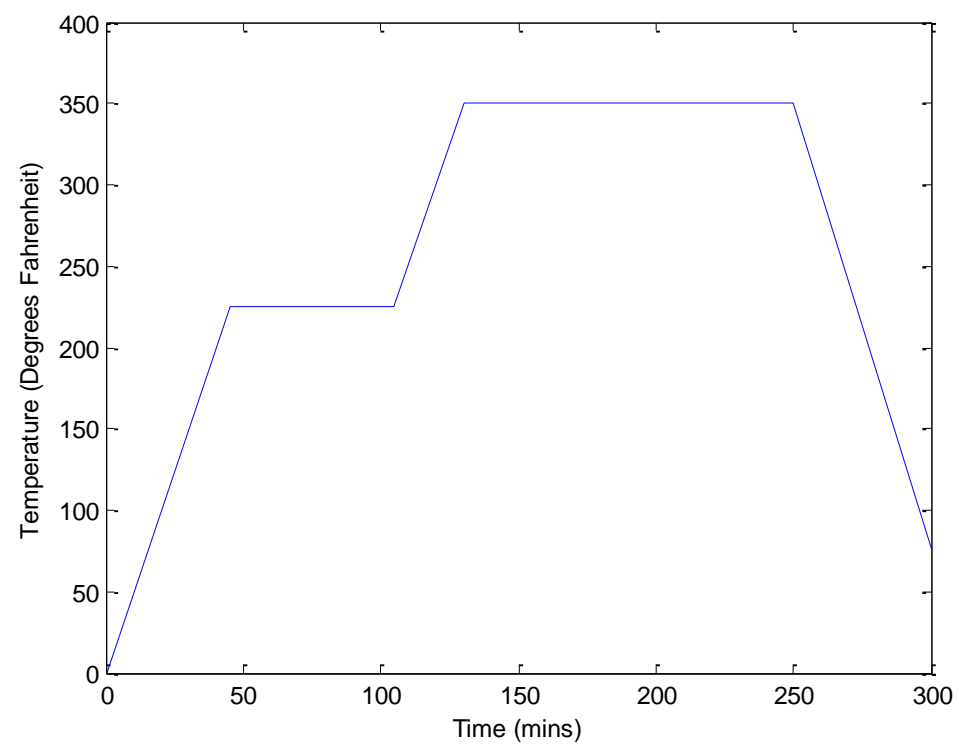

Figure 2.3 Curing Cycle for Hexply 6376 A280-5H

The carbon fiber was cured with pressure set at 2 psi to allow the honeycomb to indent into the carbon fiber during cure to increase bonding surface area between core and face sheet. The pre-preg is cut into 12 "x 12 " squares and placed between two layers of non-porous material. The stacking of the layers is as follows: non-porous material, six layers of bi-directional A280-5H pre-preg carbon fiber, and non-porous material. The non-porous material is essential to keep the heating press clean from resin and to allow for easy removal of the cured carbon fiber plate. It is imperative that the carbon fiber sheets be layered on each other at right angles. Small changes in ply orientation can result in 
unsymmetrical laminates and subsequently skewed results in mechanical tests. The carbon fiber plate was then cut into 10 inch long by 1 inch wide by 0.1 inch thick or wider inch strips to comply with ASTM D3039 tensile testing. ${ }^{30}$ Cutting specimens to the required dimensions required a tile saw with accurate measuring device to ensure repeatability between samples. In accordance with ASTM standard, aluminum tabs, also known as grips, measuring 1"x1" are used for the tensile testing shown in Figure 2.3.

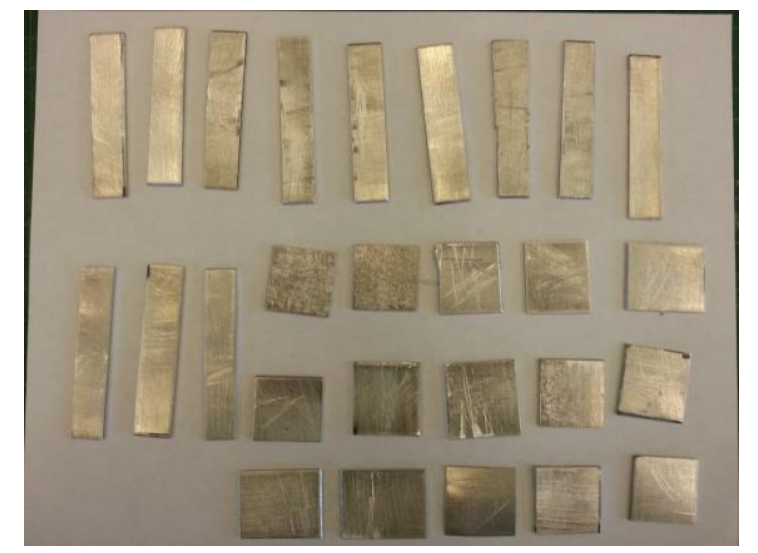

Figure 2.4. Scored aluminum tabs used for tensile tests.

The tabs were scored to allow for better grip between the carbon fiber surface and aluminum tab. Scoring the aluminum increases the bonded surface and thus bonding strength. The tabs were cleaned and attached to the carbon fiber samples with a resin-epoxy adhesive shown Figure 2.4. The curing cycle for the structural adhesive can be seen in figure 2.5 . Temperature is ramped at $5 \% / \mathrm{min}$ up to $120^{\circ} \mathrm{F}$, held at that temperature for 1 hour and then ramped down at a rate of $5 \% / \mathrm{min}$ back to room temperature. 
Grips apply sufficient lateral pressure to

prevent slippage between the grip face and the test specimen. A strain transducer in the lateral direction of the test samples is needed to determine poisons ratio. The Longitudinal strain is calculated in the Instron testing machine and thus a strain gage was not necessary in the longitudinal

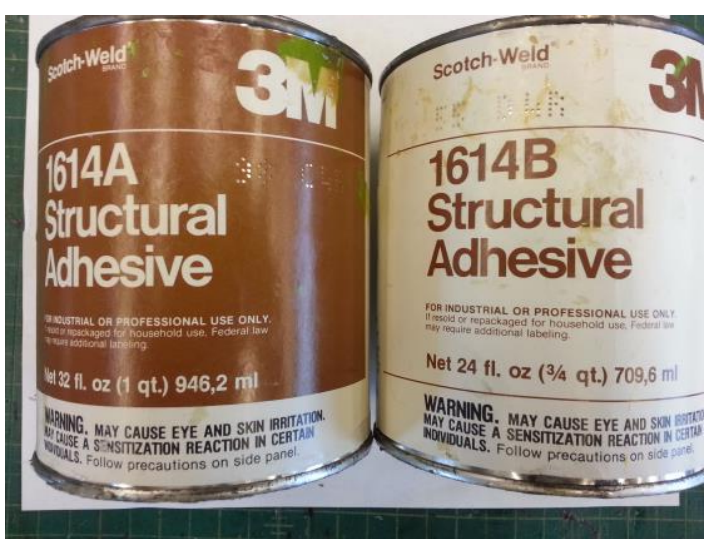

Figure 2.5. 1614 A\&B Structural adhesive from 3M used on tensile testing tabs

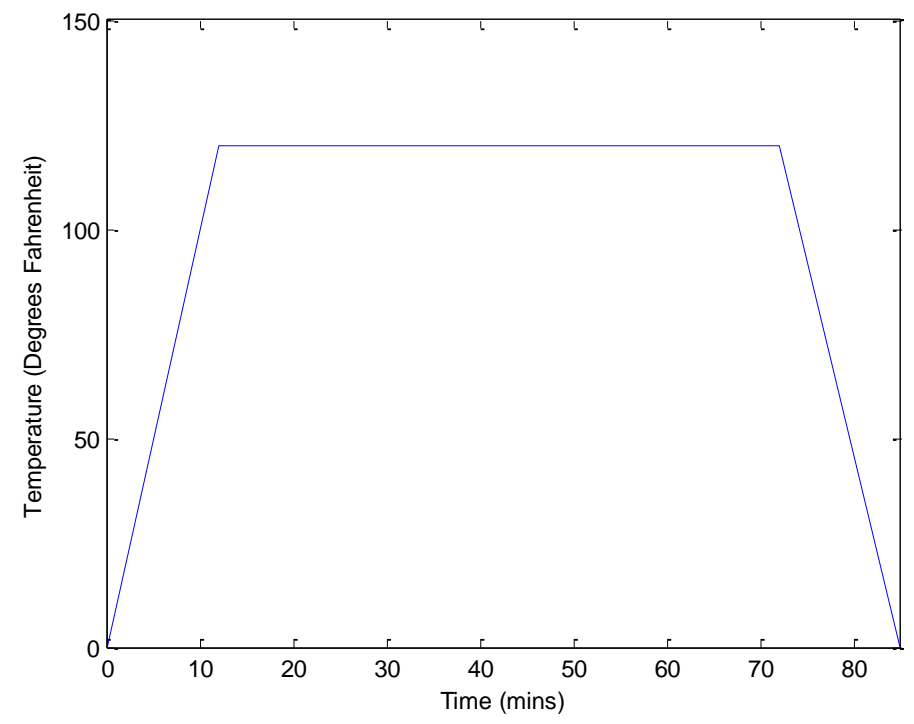

Figure 2.6. Cure Cycle for Structural Adhesive

direction, shown in Figure 2.7. Strain gauges should be placed in the middle of the test sample. Consideration of temperature compensation is recommended for non-ambient temperature environments. 
To meet ASTM standards at least six samples were needed for testing. Each sample strip is first

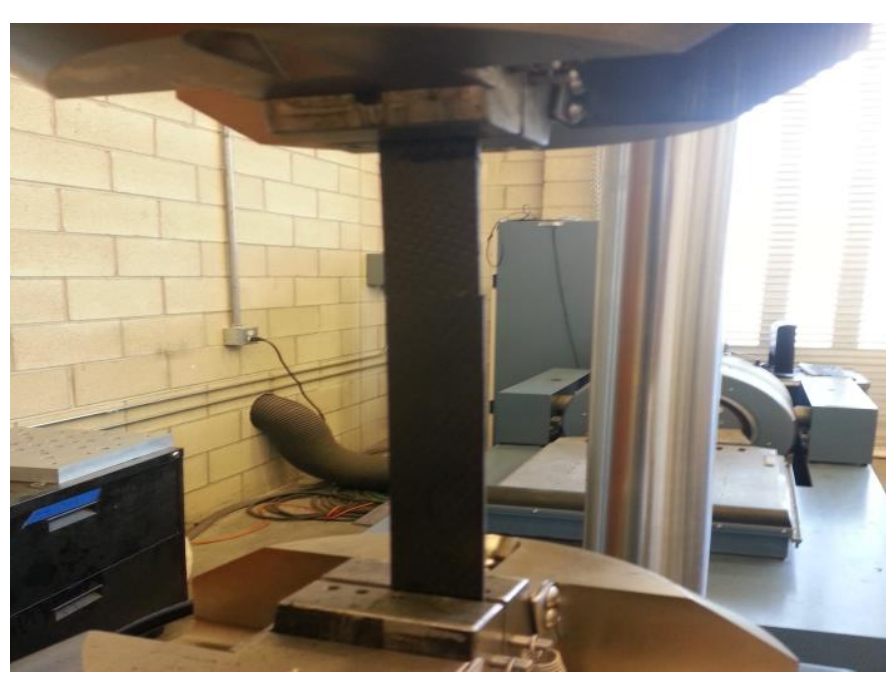

Figure 2.7. Tensile Testing of Carbon Fiber fed into the top fixture of the Instron 8801 machine and clamped down while being positioned perfectly vertical. The bottom fixture is moved into position to reach the bottom tab of the sample and then allowed to clamp down. Care must be taken to make sure there is no twist to the sample strips as it will induce a bending moment during the test and will greatly affect

results, shown in Figure 2.8.

As per ASTM D3039, A standard head displacement rate of $0.05 \mathrm{in} . / \mathrm{min}$ was applied to specimens until failure. The Instron machine records all testing data: elongation, load, stress, strain, and ultimate stress. Test specimens that failed near the grip fixture were discarded from results.

The raw data is then analyzed to obtain ultimate tensile strength and Young's modulus. Calculation of the ultimate tensile strength is executed through Eq 2.1. Determination of the tensile stress at each required data point is

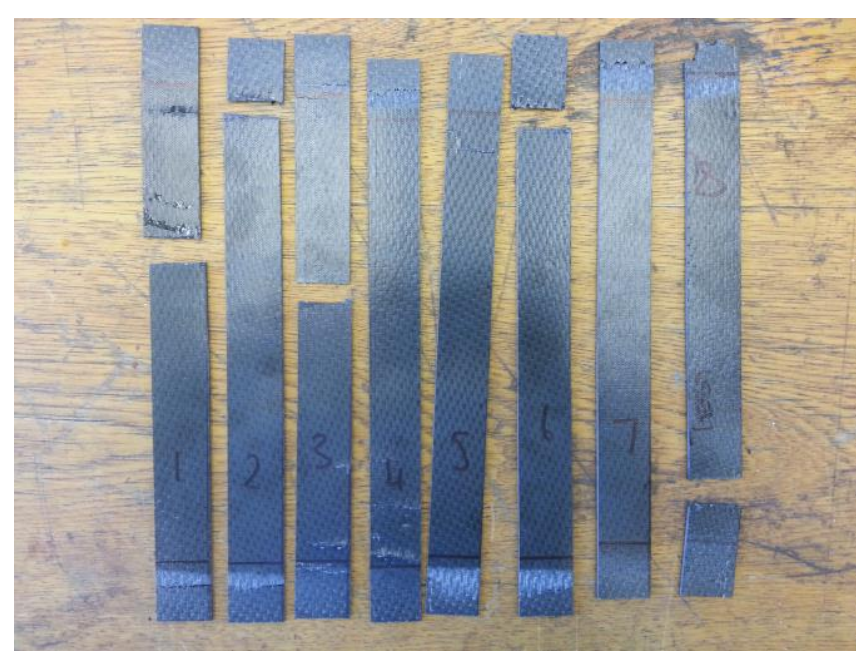
executed through Eq 2.2.
Figure 2.8. Samples failing at or near grip fixture are discarded 


$$
\begin{aligned}
F^{u l t} & =\frac{P^{\max }}{A} \\
\sigma_{i} & =\frac{P_{i}}{A}
\end{aligned}
$$

Where $F^{\text {ult }}=$ ultimate tensile strength, [Mpa [psi]; $P^{\max }=$ maximum force before failure, $\mathrm{N}$ [lbf]; $\sigma_{i}=$ tensile stress at ith data point, Mpa [psi]; $P_{i}=$ force at $\mathrm{i}^{\text {th }}$ data point, $\mathrm{N}$ [lbf]; and $A=$ average crosssectional area, $\mathrm{mm}^{2}\left[\right.$ in. $\left.^{2}\right]$.

$$
E=\varepsilon / \sigma
$$

The elastic modulus is calculated in the linear elastic region between $10-25 \%$ of ultimate load for each specimen and then averaged resulting in an elastic modulus. In addition to the elastic moduli, the ultimate stress values were also recorded for each sample. Ultimate stress is needed for theoretical calculations for the sandwich structure. Table 2.2 shows the tensile mechanical properties found experimentally. Experimental tensile data can be found in the appendix. A total of 12 tensile specimens were tested with 7 acceptable failure modes.

Table 2.2. Tensile mechanical properties of Hexply 6376 A280-5H carbon fiber pre-preg

\begin{tabular}{|l|l|l|}
\hline & $\begin{array}{l}\text { Young's } \\
\text { Modulus (Msi) }\end{array}$ & $\begin{array}{l}\text { Ultimate } \\
\text { Tensile } \\
\text { Strength (ksi) }\end{array}$ \\
\hline Experimental & $12.934 \pm .263$ & $163.3 \pm 5.22$ \\
\hline Data Sheet & 9.7 & 127 \\
\hline Error (\%) & $-33.3 \%$ & $-28.6 \%$ \\
\hline
\end{tabular}

The tensile tests produce conflicting results with the data sheet values, which leads me to believe the material may have been labelled wrongly. 


\subsubsection{Compressive Mechanical Properties}

As per ASTM D6641 the composite face sheets are cut into strips 5.5 inches long by 0.5 inches wide by approximately 0.1 in thick, equivalent to 7 plies of HexPly 6367, to obtain compressive property data. Laminate compressive strength and modulus can be calculated as follows:

$$
\begin{gathered}
h \geq \frac{l_{g}}{0.9069 \sqrt{\left(\left(1-\frac{1.2 F^{c u}}{G_{x z}}\right)\left(\frac{E^{c}}{F^{c u}}\right)\right)}} \\
F^{c u}=\frac{P_{f}}{w h} \\
E^{c}=\frac{P_{2}-P_{1}}{\left(\varepsilon_{x 2}-\varepsilon_{x 1}\right) w h}
\end{gathered}
$$

Where: $F^{c u}=$ laminate compressive strength, psi, $l_{g}=$ length of gage section, $0.5 \mathrm{in}, G_{x z}=$ through thickness shear modulus, psi, $P_{f}=$ maximum load to failure, lbf, $w=$ specimen gage width, in, $h=$ specimen gage thickness, in, $E^{c}=$ compressive modulus, psi, $P_{2}, P_{1}=$ load at $\varepsilon_{x 2}, \varepsilon_{x 1}$, lbf, $\varepsilon_{x 2}, \varepsilon_{x 1}=$ actual

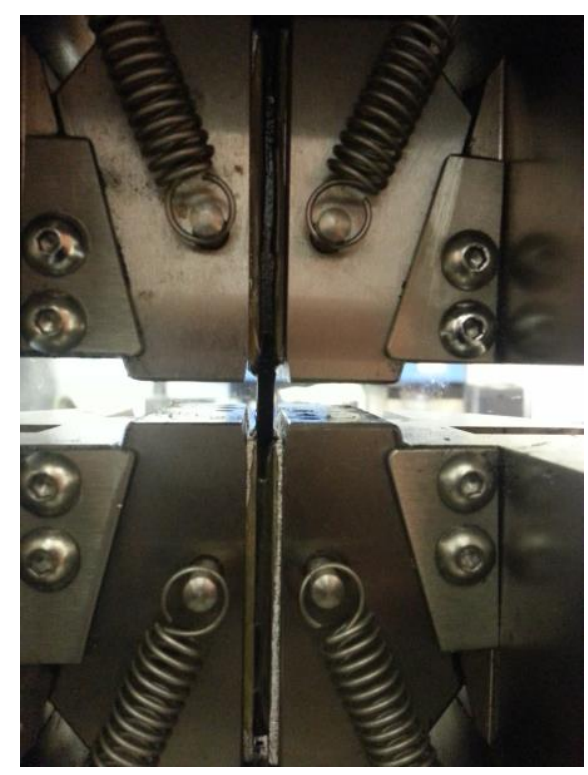

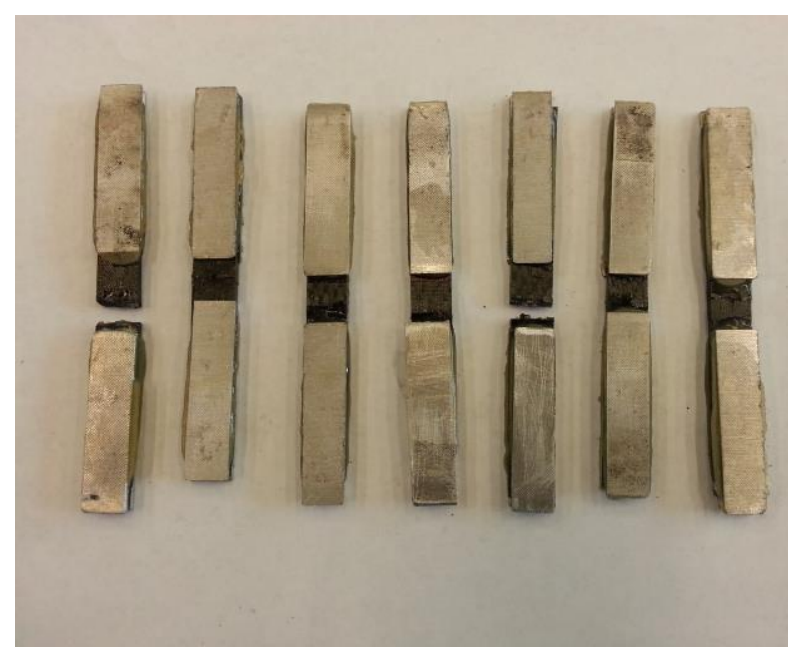

Figure 2.10. Broken Compressive Specimens.

Figure 2.9. Compressive specimen in the Instron Machine.

strain nearest upper, lower end of strain range used. Results of compressive tensile testing can be seen 
in table 2.3. An example of the experimental test set-up can be seen in Figure 2.9. A total of 12 specimens were tested, 8 had acceptable failure modes which can be seen in Figure 2.10. Experimental data can be seen in the appendix.

Table 2.3. Compressive Mechanical properties of Hexply 6376 A280-5H Carbon Fiber.

\begin{tabular}{|c|c|c|}
\hline & $\begin{array}{l}\text { Compression } \\
\text { Young's } \\
\text { Modulus (Msi) }\end{array}$ & $\begin{array}{c}\text { Compressive } \\
\text { Ultimate } \\
\text { Strength (ksi) }\end{array}$ \\
\hline Experimental & $4.48 \pm 0.4$ & $127.7 \pm 3.4$ \\
\hline Datasheet & 9.5 & 134 \\
\hline Error (\%) & $52.8 \%$ & $4.93 \%$ \\
\hline
\end{tabular}

It must be noted that compressive and tensile data found experimentally are off by a large margin and have produced results not typical of a bi-weave carbon fiber. For analysis purposes, experimental mechanical properties shall be used.

\subsubsection{Poisson's Ratio of Face Sheets}

To determine the Poisson's ratio of the face sheets ASTM E132 is used..$^{31}$ ASTM E132 is used in conjunction with ASTM D3039 and follows the same fabrication and test method. The only difference is

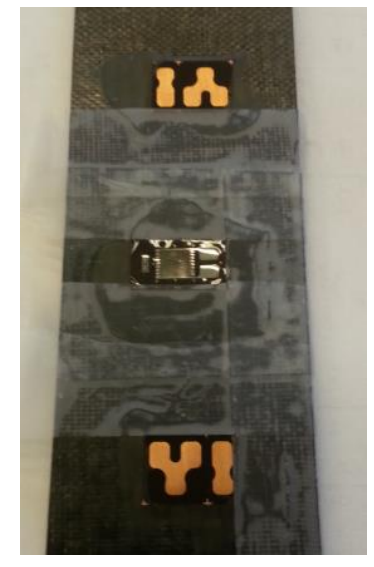

Figure 2.11. Placement of transverse strain gage that strain gages are added to the test specimens to obtain the twodimensional behavior of the lamina. To do so, an M-Bond 200 Adhesive kit is used to adhere the gage to the specimen. The surface of the composite sample needs to be cleaned with acetone before gage application for optimal adhesion. The resin from the kit is applied to the composite surface and allowed to dry for a few seconds. The hardener is applied to the gage and applied to the composite sample on the catalyst 
surface. Pressure is applied over the work area for a few minutes for the epoxy to cure. Once cured the strain gage must be soldered and resistance verified with requirements. The gage system is shown in

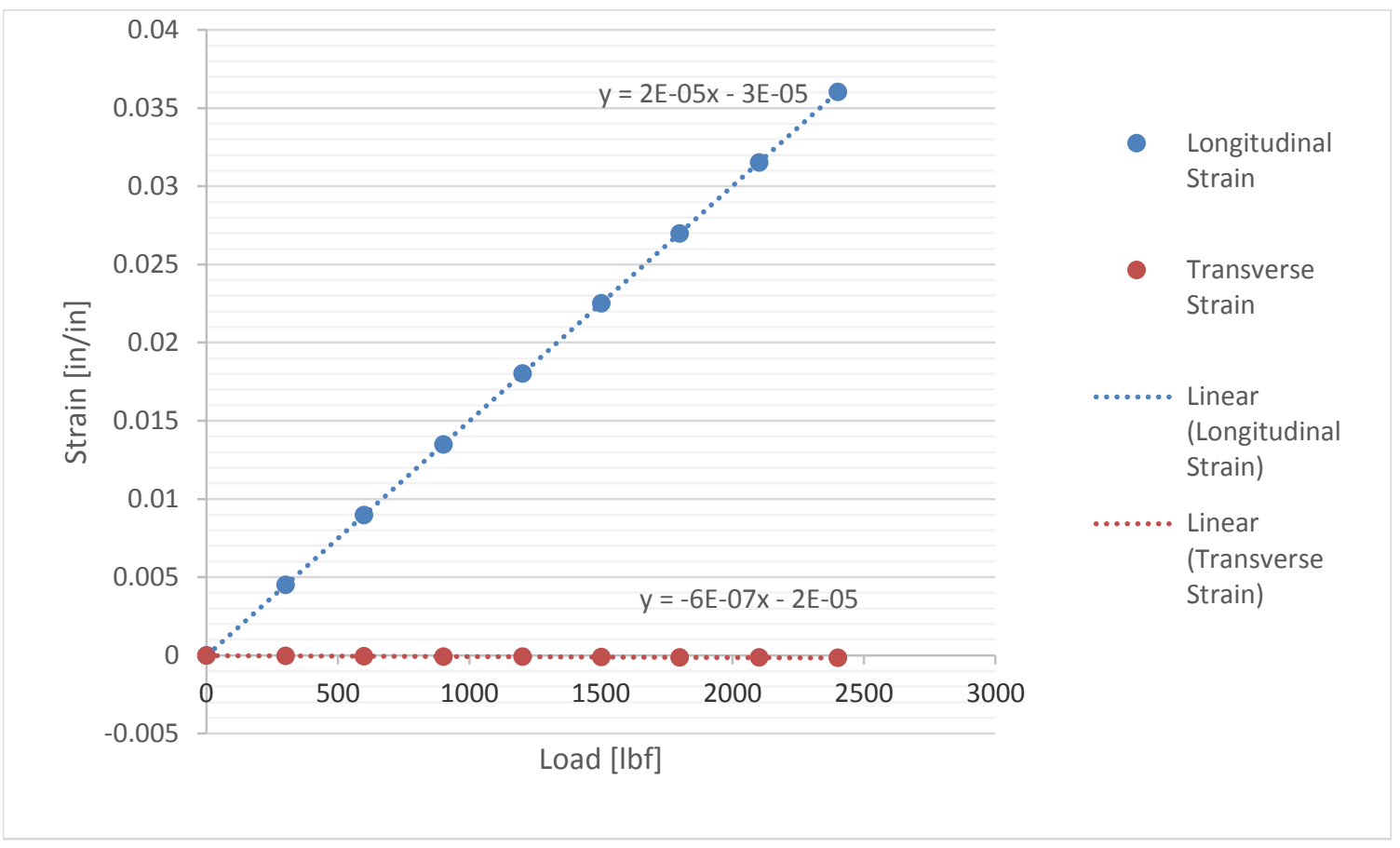

Figure 2.12. Strain versus Load in longitudinal and transverse directions of the Carbon Fiber

Figure 2.8. To obtain values for Poisson's ratio, a plot of the average strain versus load was created for both longitudinal and transverse directions as shown in Figure 2.12.

The derivative of the strain in the transverse direction is divided by the derivative of the strain in the longitudinal direction as seen in Eq. 2.4.

$$
v=\frac{-\frac{\partial \varepsilon_{T}}{\partial P}}{\frac{\partial \varepsilon_{L}}{\partial P}}=-\frac{\varepsilon_{T}}{\varepsilon_{L}}
$$

Where $\mathrm{v}$ is the Poisson's ratio, $\mathrm{P}$ is the applied load and $\varepsilon_{\mathrm{T}}$ and $\varepsilon_{\mathrm{L}}$ are the transverse and longitudinal strains respectively. A plot of the strain versus load curve for the average transverse and longitudinal directions can be seen in Figure 19. Poisson's ratio for Hexply 6367 A280-5H was found to be 0.03 , which is a little low for bi-directional carbon fiber sheet, but not terrible. Hexcel does not provide Poisson's ratio for Hexply 6367 A280-5H carbon fiber and is thus not shown. 


\subsubsection{Weight Fraction and Density of Face Sheets}

The density of the face sheet is required in this research particularly for theoretical and finite element analysis. Experimental density values are found by measuring the dimensions of a sample and the samples weight. To find the density of the HexPly 6376 A280-5H carbon fiber face sheets, cured samples of 4, 6 and 8 layers were cut into $4 "$ x 1 " rectangles and measured with a micrometer along with the samples' weight. Each configuration contained 6 samples for statistical accuracy. The density is calculated by dividing the mass sample in Ibs. by the mass volume in $\mathrm{in}^{3}$. Validation of experimental density, the theoretical value was calculated using the densities of the fibers and matrix along with the volume fraction of the laminate. The weight fraction requires the measured weights of the specimen before and after ignition loss. The weight fraction is defined as W and subscripts are dependent on constituent material, $f$ for fiber, $m$ for epoxy. The weight ratio is the ratio of component to the whole structure and is defined as follows:

$$
\begin{aligned}
& W_{\text {total }}=w_{f}+w_{m} \\
& W_{m}=\frac{\left(w_{t o t a l}-w_{f}\right)}{w_{t o t}} \\
& W_{f}=\frac{w_{f}}{w_{t o t}}
\end{aligned}
$$

The volumetric fractions require the density of each constituent material. The volume fraction is defined as $V$ and is as follows:

$$
\begin{aligned}
& v_{\text {total }}=v_{f}+v_{m} \\
& V_{m}=\frac{\left(v_{t o t a l}-v_{f}\right)}{v_{t o t}} \\
& V_{f}=\frac{v_{f}}{v_{t o t}}
\end{aligned}
$$


The relationship between volumetric fraction and weight fraction is dependent on the density of the constituent materials and structure as a whole. The proportional relationship of the weight fractions to volumetric fractions is as follows:

$$
\begin{aligned}
& V_{f}=\frac{\rho_{\text {total }}}{\rho_{f}} W_{f} \\
& V_{f}=\frac{\rho_{\text {total }}}{\rho_{m}} W_{m}
\end{aligned}
$$

If the density of the structure is unknown, the volumetric fractions can be determined with the weight of the constituent materials only as follows:

$$
\begin{aligned}
& V_{f}=\frac{\rho_{m} W_{f}}{\rho_{f} W_{m}+\rho_{m} W_{f}} \\
& V_{m}=1-V_{f}
\end{aligned}
$$

The density of the Hexply 6376 matrix epoxy and A280-5H Fibers are given by Hexcel and are 0.0473 and $0.0648 \mathrm{lbs} . / \mathrm{in}^{3}$ respectively. Using the $55.29 \%$ fiber volume fraction stated on the Hexcel data sheet, Eq. 2.22 can be used to determine the density of the laminate:

$$
\rho_{\text {laminate }}=V_{\text {fibers }} * \rho_{\text {fibers }}+V_{\text {matrix }} * \rho_{\text {matrix }}
$$

Table 2.4 shows the results of the density calculations.

Table 2.4. Density Calculations of cCrbon Fiber

\begin{tabular}{|c|c|c|}
\hline Configuration & $\begin{array}{c}\text { Density } \\
\left(\mathbf{l b s} / \mathbf{i n}^{\mathbf{3}}\right)\end{array}$ & Standard Deviation $\left(\mathbf{l b s} / \mathbf{i n}^{\mathbf{3}}\right)$ \\
\hline 4 Layers & 0.0562 & 0.0013 \\
\hline 6 Layers & 0.0542 & 0.0011 \\
\hline 8 Layers & 0.056 & 0.0011 \\
\hline Average & $\mathbf{0 . 0 5 5 5}$ & 0.0014 \\
\hline Theoretical & $\mathbf{0 . 0 5 7}$ & - \\
\hline Error (\%) & $2.63 \%$ & \\
\hline
\end{tabular}


Experimental volumetric fiber burning density calculations showed favorable results with data sheet values.

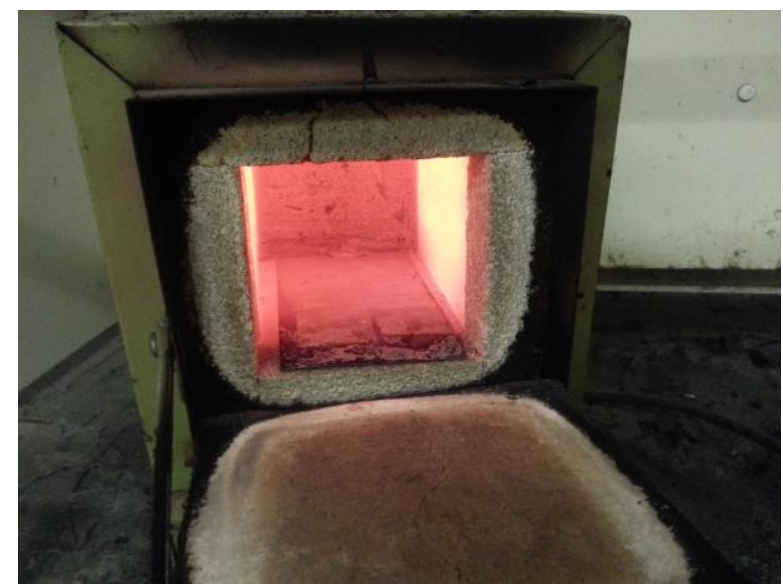

Figure 2.13. Crucible used for ignition loss testing.

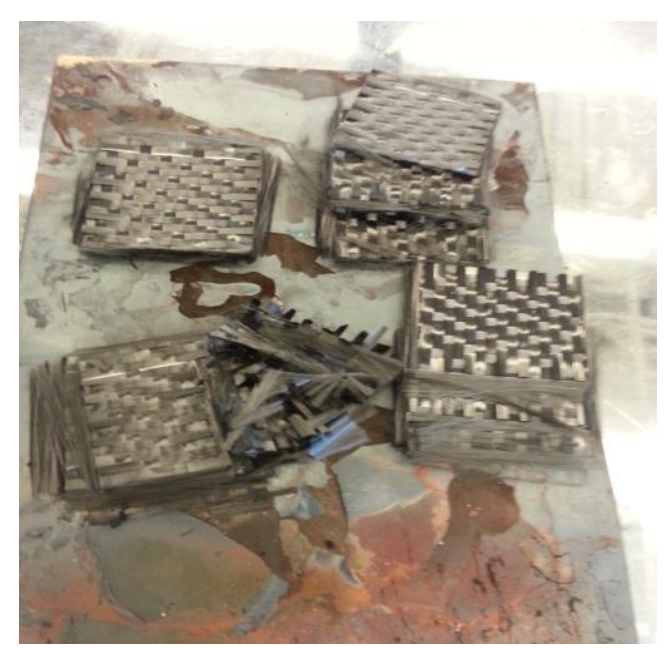

Figure 2.14. Carbon fiber samples after ignition

To validate the $55.29 \%$ fiber volume fraction stated by Hexcel, ASTM D2584 ${ }^{34}$ is used to measure the ignition loss of a cured reinforced resin sample. The 4" by 1 " samples used for density calculations were cut into 1 " $\times 1$ 1" squares and reweighed. One sample consisted of $4,1^{\prime \prime} \times 1$ " squares and were placed into a crucible shown Figure 2.13 and maintained at a temperature of $1050 \pm 50^{\circ} \mathrm{F}$ for approximately one hour to completely burn off the epoxy, leaving only the carbon fibers shown Figure 2.14 .

The ignition loss of the specimen in weight percent is calculated as follows:

$$
\text { Ignition loss, weight } \%=\left[\frac{W_{1}-W_{2}}{W_{1}}\right] * 100
$$

Where: $W_{1}=$ weight of specimen, lbs and $W_{2}=$ weight of residue, lbs.

The fiber volume fraction of the samples can be calculated as follows:

$$
V_{\text {fiber }}=\frac{\frac{w_{\text {laminate }}}{\rho_{\text {laminate }}}-\frac{w_{\text {matrix }}}{\rho_{\text {lmatrix }}}}{\frac{w_{\text {laminate }}}{\rho_{\text {laminate }}}}
$$


Where $\mathrm{m}$ donates mass of constituent in Ibs. The results can be seen in Table 2.5.

Table 2.5. Weight fiber fractions calculated experimentally and theoretically

\begin{tabular}{|c|c|}
\hline & Volumetric Fiber Fraction \\
\hline Experimental & $0.5571 \pm 9.4 \%$ \\
\hline Theoretical & 0.5529 \\
\hline Error (\%) & $0.759 \%$ \\
\hline
\end{tabular}

\subsection{Honeycomb Core Mechanical Properties}

Determination Honeycomb core mechanical properties proved difficult due to not having access to the required test apparatus's for testing under ASTM C273 ${ }^{37}$, and tests to determine Young's moduli. Although core shear strength, and core shear modulus could not be obtained experimentally and from the limited experimental mechanical properties, the required mechanical properties were obtained theoretically. 


\subsubsection{Poisson's ratio}

ASTM standard D6790 $-02^{38}$ was used for determination of honeycomb Poisson's ratio in both longitudinal and transvers core orientations from the anticlastic curvature radii shown in Figure 2.1.

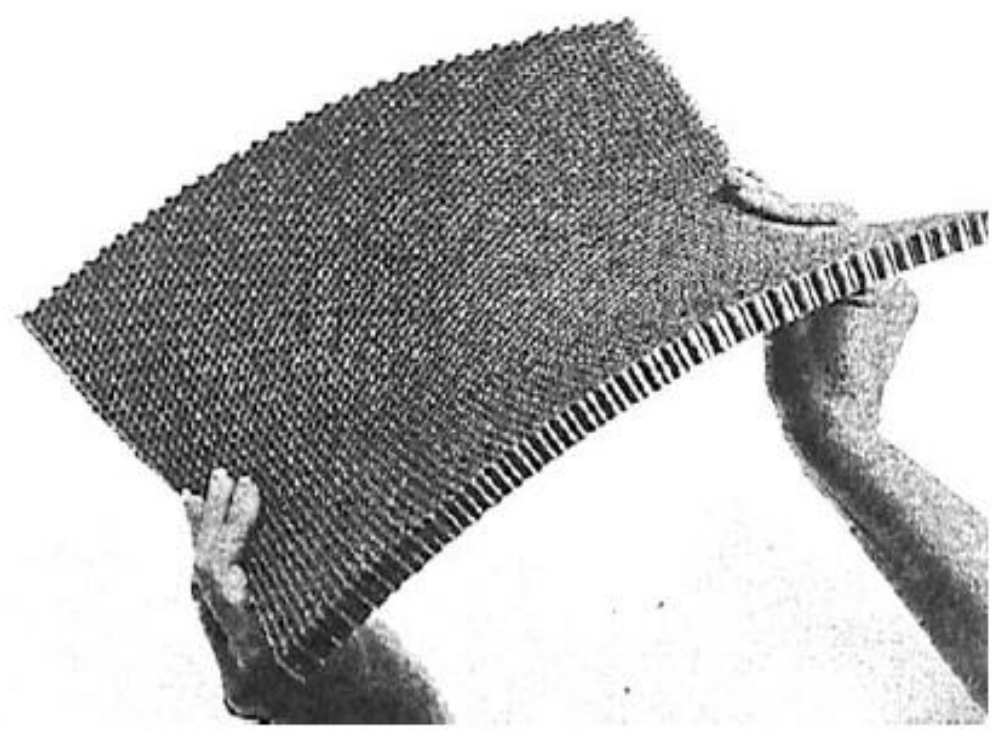

Figure 2.15 Anticlastic Curvature ${ }^{38}$

Certain sandwich panel finite element programs require the Poisson's ratio of the honeycomb core. As it is not possible to measure honeycomb's Poisson's ratio by standard method, the test method ASTM D6790 is on means of obtaining the Poisson's ratio of honeycomb, however this test method is not widely used and is in its conceptual stage. A specimen of 12 by 12 inches is bent in both directions, as shown in Figure 2.16 around a 24 -inch drum and the anticlastic curvature radius is calculated as follows.

$$
\begin{gathered}
R_{a}=\frac{4 d^{2}+c^{2}}{8 d} \\
\mu=\frac{R_{c}}{R_{a}}
\end{gathered}
$$


Where: $\mu$ = Poisson's ratio, $R_{a}=$ anticlastic curvature radius, $R_{c}=$ cylinder radius, $\mathrm{c}=$ chord measurement, and $d=$ depth measurement.

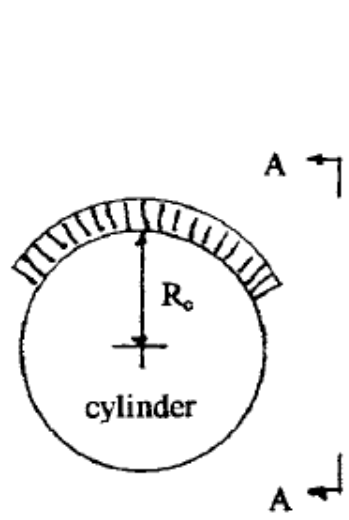

$\mathbf{R}_{\mathrm{e}}=$ cylinder radius

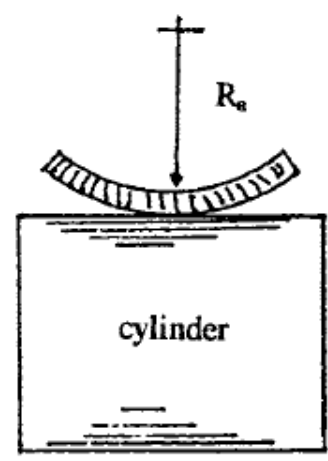

View A-A

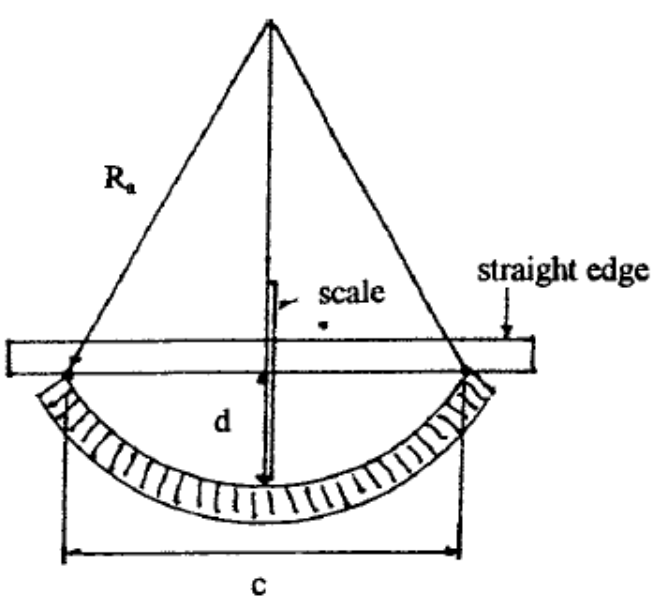

Measurements

Figure 2.16. Measurements for ASTM D6790 38

From the Poisson's ratio and shear modulus given by Hexcel, Young's moduli in the transverse and longitudinal directions can be determined as follows:

$$
E_{L, T}=G_{L, T}\left(2\left(1+v_{L, T}\right)\right)
$$

In total 8 specimens were tested in both longitudinal and transverse directions. Table 2.6 shows the results of the testing.

Table 2.6. Experimental Mechanical Properties of Hexcel HRH-10-1/3-5 Honeycomb

\begin{tabular}{|c|c|c|c|}
\hline Direction & Poisson's & $\begin{array}{c}\text { Young's } \\
\text { Moduli } \\
\text { (psi) }\end{array}$ & $\begin{array}{c}\text { Shear } \\
\text { Modulus } \\
\text { (psi) }\end{array}$ \\
\hline Longitudinal & 0.8036 & 37669 & 10442.7 \\
& \pm 0.033 & \pm 719 & \\
\hline Transverse & 0.4114 & 16375 & 5801.5 \\
& \pm 0.0066 & \pm 76.6 & \\
\hline
\end{tabular}


Experimental values cannot be compared to data sheet values, as Hexcel has not supplied them.

\subsection{Composite Sandwich panels}

This section covers the mechanical behaviors of the composite sandwich specimens. The mechanical properties found in this section are determined using equations presented in relevant ASTM standards using experimental data.

\subsubsection{Necessity of Adhesive Layer between Core and Face Sheet}

A similar method to the face sheets is used to determine the mechanical properties of the adhesive. An adhesive with similar curing cycles to the carbon fiber face sheets was used to prevent

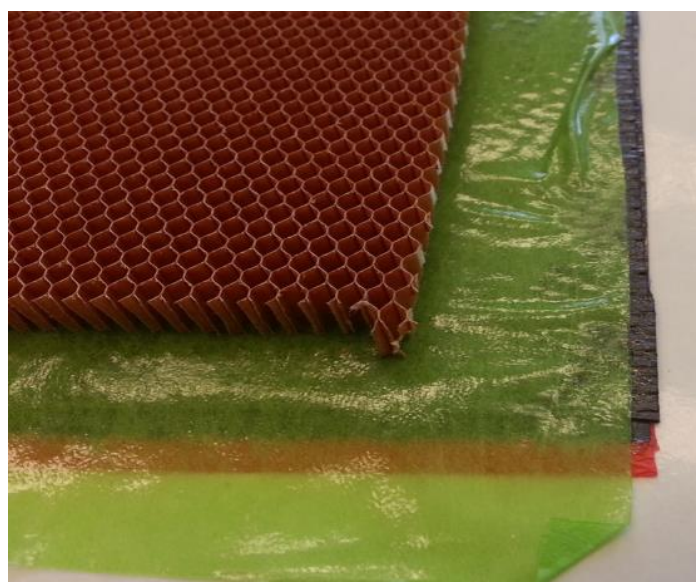

Figure 2.17. Viscid side of adhesive placed against honeycomb core. shock, or over cure of the adhesive. The adhesive chosen was 3M's Scotch-Weld Structural Adhesive

Film 163-2OST. The adhesive is green in color weighing $0.03 \mathrm{lb} / \mathrm{ft}^{2}$ with a nominal thickness of 5.5 mils. ${ }^{35}$ The $163-20 S T$ has a range of cure cycles from $225^{\circ} \mathrm{F}$ at 90 minutes to $300^{\circ} \mathrm{F}$ at 60 minutes. To minimize error caused by different cure cycles, the whole panel cure cycle was set to the carbon fiber face sheet cure cycle seen in the above section 2.1.

The manufacture of test specimens follows ASTM D638 standards. ${ }^{36}$ When making the sandwich; the viscid side of adhesive is placed against the honeycomb so to prevent face sheet slipping if pre-cured sandwich is to be stored before curing as shown in Figure 2.17. 


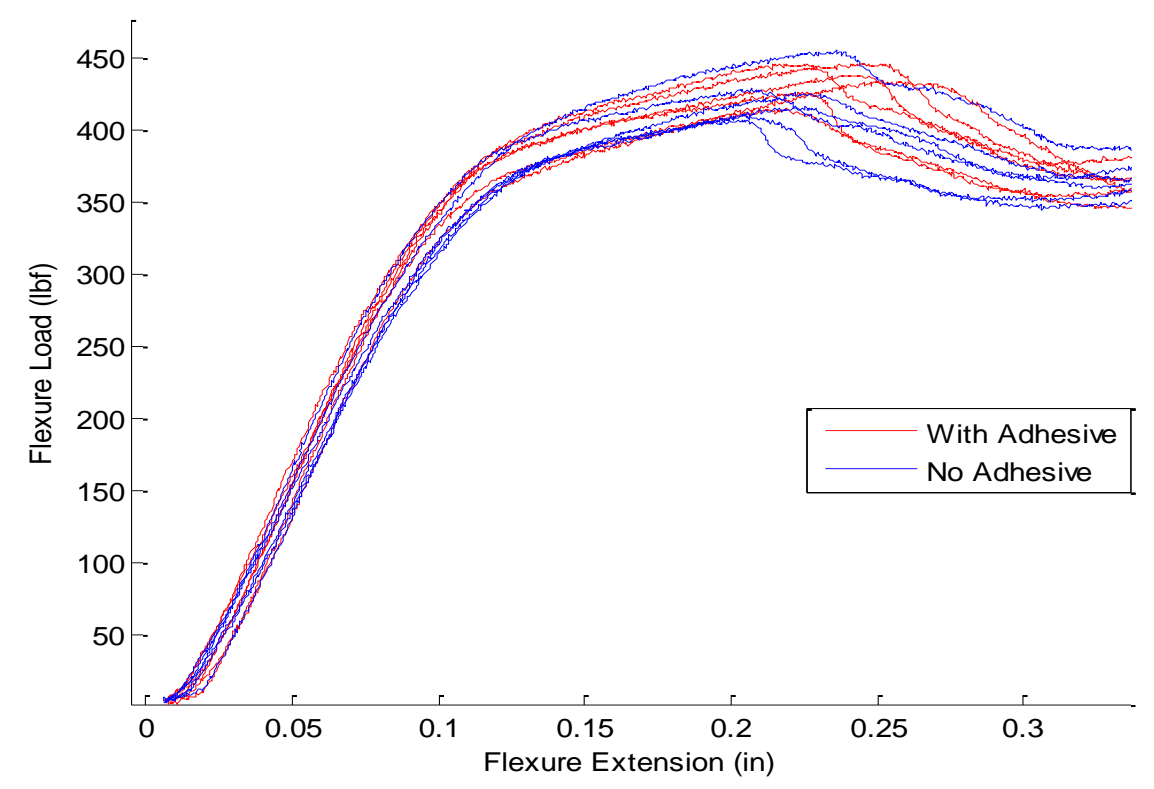

Figure 2.18. Load versus Extension plot for 3 ply Sandwich structures with and without adhesive layers

To test the effects of an adhesive layer between the face sheets of the sandwich and the honeycomb core, 6 sandwiches with an adhesive layer between core and face sheets and 6 sandwiches without an adhesive layer were constructed and tested under three-point bending. Figure 2.18 and Table 2.7 show that sandwich structures with the adhesive layers exhibited marginally lower ultimate loads than sandwich structures without adhesive whilst incurring a small weight increase. For the purposes of optimization in this thesis, it was determined that the adhesive layer does nothing to increase the performance of the sandwich structure.

Table 2.7. Effect of adhesive layer on the ultimate load at initial failure

\begin{tabular}{|c|c|c|}
\hline & $\begin{array}{c}\text { Ultimate Load } \\
\text { (lbs) }\end{array}$ & $\begin{array}{c}\text { Standard } \\
\text { Deviation (lbs) }\end{array}$ \\
\hline With Adhesive & 424.37 & 17.502 \\
\hline Without Adhesive & 434.44 & 12.263 \\
\hline
\end{tabular}




\subsubsection{Core Shear Properties of Sandwich Constructions by Beam Flexure}

This test covers determination of the core shear properties of flat sandwich constructions subjected to flexure, such that the applied moments produce curvature of the sandwich facing planes. All specimens should be 3 inches wide, 8 inches long and face sheet to core thickness less than 0.1 to

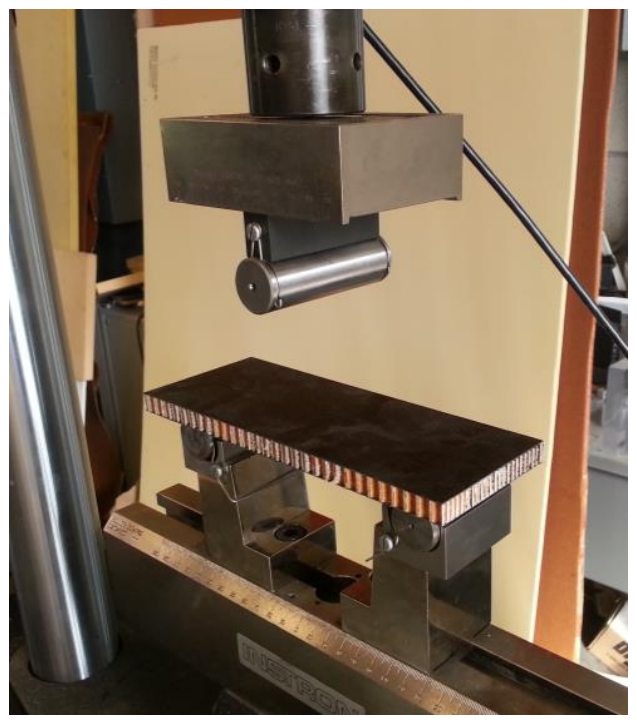

Figure 2.19. Panel placement for testing

follow ASTM C393 standards. ${ }^{39}$ All bending tests were conducted in the Instron 8801 machine at Cal Poly SLO Aero structures/composites Lab.

All specimens shall have dimensions recorded. Measure and record the length of the support and loading spans being 6 and 0 inches respectively. The speed of the testing set is to be set to 0.25 in per minute. Arrange the loading fixture as seen in Figure 2.19 and place in testing fixture. Place the specimen into the test fixture aligning the longitudinal axis perpendicular to the longitudinal axis of the loading bars. Apply the compressive force to the specimen at the specified rate of $0.25 \mathrm{in} / \mathrm{min}$. Load the specimen until failure or until the deflection equals the specimen thickness. ${ }^{39}$ Core shear ultimate stress can be calculated as follows:

$$
F_{s}^{u l t}=\frac{P_{\max }}{(d+c) b}
$$


Where: $F_{S}^{u l t}=$ core shear ultimate strength, $\mathrm{psi}, P_{\max }=$ maximum force prior to failure, $\mathrm{lb}, \mathrm{t}=$ nominal facing thickness, in, $d=$ sandwich thickness, in, $c=$ core thickness, in and $b=$ sandwich width, in.

Facing stress can be calculated using Eq. 2.29. It must be noted that Eq. 2.29 can only be used for sandwich structures with facings of equal thickness:

$$
\sigma=\frac{P_{\max } S}{2 t(d+c) b}
$$

Where: $\sigma=$ facing stress, $\mathrm{psi}, \mathrm{t}=$ facing thickness, in, and $\mathrm{S}=$ support span length, in.

For sandwich structures of unequal facing thickness, in accordance with ASTM D7249 ${ }^{40}$, the upper and lower facing stresses can be calculated as follows.

$$
\begin{aligned}
& F_{\text {upper }}=\frac{P(S-L)}{2(d+c) b t_{1}} \\
& F_{\text {lower }}=\frac{P(S-L)}{2(d+c) b t_{2}}
\end{aligned}
$$

Where: $F_{\text {upper }}=$ facing 1 stress, psi, $F_{\text {lower }}=$ facing 2 stress, psi, $t_{1}=$ upper facing thickness, in, $t_{2}=$ lower facing thickness, in, and $L=$ loading span length, in. $L=0$ for 3-point loading.

\subsubsection{Determination of Sandwich Beam Flexural and Shear Stiffness}

Testing under ASTM D7250 ${ }^{41}$ determines the flexural and transverse shear stiffness properties of flat sandwich constructions that have equal top and bottom facing thicknesses subjected to flexure such that the applied moments produce curvature of sandwich facing planes. Flexural stiffness, shear rigidity, and core shear modulus can be calculated as follows:

$$
\begin{gathered}
D=\frac{E_{f}\left(d^{3}-c^{3}\right) b}{12} \\
U=\frac{P(S-L)}{4\left(\Delta-\left(\frac{P\left(2 S^{3}-3 S L^{2}+L^{3}\right)}{96 D}\right)\right)}
\end{gathered}
$$

Where: $\mathrm{D}=$ flexural stiffness, $\mathrm{Ib}-\mathrm{in}^{2}, \mathrm{U}=$ transverse shear rigidity, lbs., $\Delta=$ beammid-span deflection, in, $\mathrm{P}=$ total applied force, Ibf. 
Equation 2.33 can then be substituted into Eq.2.34 to obtain the core shear modulus, $\mathrm{G}$, as

shown:

$$
G=\frac{U(d-2 t)}{(d-t)^{2} b}
$$




\subsection{Theoretical Analysis}

This section focuses on the development of simplified expressions for shear and flexural stiffness' in the longitudinal orientation in order to estimate deflections to reasonable accuracy. Equations using Classical beam and plate theory are compared with the more in-depth orthotropic composites approach and then compared with the FEA solver Abaqus. The comparisons of equations are shown for a better understanding of how the solver functioned and which approach is more accurate when compared to the experimental results.

A sandwich beam can be predicted rather well with the classical theory. To study local effects on the other hand, higher-order theories have to be used. The examples show that the higher-order theory gives the best descriptions, since it takes influences through the thickness of the sandwich into account. From this point of view the higher-order theory is preferable. The only disadvantage is that the derivations are more complex than those for the superposition approach. But since general numerical procedures are developed to solve the boundary value problem easily, the higher-order theory is preferable. A simple analytical expression to estimate the flexural rigidity (EI or D) and Shear stiffness (GA or $U$ ) for the sandwich structures in the longitudinal orientation is needed to predict the overall response as a ball park reference before more time and computational costly finite element analysis is conducted. This expression would act as a guide to predict the structure's stiffness without implementing all needed material properties at the lowest level required by the orthotropic composite approach.

The following assumptions are made in regards to the face sheets:

1. Orthotropic, homogenous face sheets of thickness $t_{t}$ (top face sheet) and $t_{b}$ (bottom face sheet).

2. Transverse normal stresses $\sigma_{z}$ are neglected in face sheets.

3. Transverse displacements in face sheets are independent of $z$ coordinate, such that 


$$
w_{t}=w_{t}^{0}(x, y), w_{b}=w_{b}^{0}(x, y)
$$

4. In z coordinate in the face sheets, in plane stresses, $\sigma_{x}, \sigma_{y}, \tau_{x y}$ are assumed to be linear functions.

5. In plane stresses $\sigma_{x}, \sigma_{y}, \tau_{x y}$ are neglected in the core.

6. The material properties for the face elements are known from experimental testing, including Young's modulus and shear modulus derived from Eq. 2.27

7. The cross section of the structure can be idealized as the cross section of an I-Beam.

8. Both core and face laminates contribute to the flexural stiffness (El or D). Only the core is accounted for in the shear stiffness (GA or U), due to the fact that shear deformation in the faces is negligible.

\subsection{Analytical Formulation for Mechanical Properties}

Using the simplified beam theory as proposed by Allen ${ }^{25}$ the Core Shear Stress ( 3 point loading):

$$
\tau=\frac{P_{\max }}{(d+c) b}
$$

Where $\tau$ is the core strength or shear yield stress [psi], $P$ is the load [lb], $d$ is the sandwich thickness, $c$ is the thickness of the core and $b$ is the width of the specimen. The effective area of transverse shear stress is the core thickness + half of each face sheet thickness, thus, $\frac{(d+c)}{2}=c+\frac{t_{t}}{2}+$

$\frac{t_{b}}{2}$. The sandwich beam deflection for a three-point bend test is as follows:

$$
\Delta=\frac{P L^{3}}{96 D}+\frac{P L}{4 U}
$$

Where $\Delta$ is total mid-point deflection, $D$ is the panel bending stiffness, $U$ is the panel shear rigidity and $L$ is the panel length. If the facing modulus is known and the top and bottom face sheets are identical and symmetric, the panel flexural rigidity is as follows:

$$
D=2 \frac{E_{f} t_{f}^{3}}{12}+\frac{E_{f} t_{f} d^{2}}{2}+\frac{E_{c} t_{c}^{3}}{12}=2 D_{f}+D_{o}+D_{c}
$$


Where $E_{f}$ and $E_{c}$ are the facing and core moduli, $t_{f}$ and $t_{c}$ are the facing and core thicknesses, and $\mathrm{d}$ is the distance between the centroids of the faces $\left(\mathrm{d}=\frac{\left(t_{f}+t_{c}\right)}{2}+c\right) . D_{f}, D_{o}$, and $D_{c}$ are the bending stiffness's of the faces about their individual axes, faces about the middle axis, and core, respectively. ${ }^{23}$ The panel shear rigidity is as follows:

$$
U=\frac{G_{c}(d+c)^{2} b}{4 c}
$$

Where $G_{c}$ the core shear modulus is calculated using Eq. 3.5.

$$
G_{C}=\frac{E_{c}}{2\left(1+v_{c 12}\right)}
$$

In the case of uneven face thickness' a different approach based on the assumptions in section 3 , can be utilized to obtain bending and shear stiffness'.

$$
\begin{aligned}
& E I=b D=\sum_{i=1}^{n} E_{i} I_{\text {total,part }, i} \\
& G A=U=\sum_{i=1}^{m} G_{\text {core }} A_{\text {total }, \text { core }, i}
\end{aligned}
$$

Where $\mathrm{n}$ is the total number of sub sections; $\mathrm{m}$ is the number of core subsections; $I_{\text {total,part, } i}$ is the moment of inertia of the $\mathrm{i}^{\text {th }}$ subsection about the neutral axis of the whole structure and $A_{\text {total,core }, i}$ is the are of the core $\mathrm{i}^{\text {th }}$ subsection.

In order to obtain $I_{\text {total,part }, i}$ the centre of gravity of the whole section is needed and is calculated as follows:

$$
C_{g}=\frac{\sum A_{i} y_{c, i}}{A_{\text {total }}}
$$

Where $y_{c, i}$ is the distance from the bottom of the whole section to subsection centre.

The moment of inertia of the whole section is equal to sum of the subsections about their own axis and using the parallel axis theorem.

$$
I_{i}=\frac{1}{12} b h^{3} \text { (Beam Element) }
$$




$$
\begin{aligned}
& I_{i}=\frac{1}{12\left(1-v_{12}^{2}\right)} b h^{3} \text { (Plate Element) } \\
& I_{\text {total,part }, i}=\sum_{i} I_{i}+\sum_{i}\left(y_{i}-C_{g}\right)^{2} A_{i}
\end{aligned}
$$

By substituting Eq. 3.5-11 into 3.2 theoretical midspan beam deflection can be estimated. The above equations assume symmetry about the panels' middle axis and thus deflection to the extensional bending coupling or B matrix is assumed to be 0 . As the top to bottom face sheet thickness becomes too small or large, the extensional-bending coupling cannot be neglected and can be calculated as follows. Starting with the three dimensional thermoelastic strain-stress relation for anisotropic material, this is given as:

$$
\varepsilon_{i}=S_{i j} \sigma_{i j}
$$

Where the total strain, $\varepsilon_{i}$, is the sum of the mechanical strain, $S_{i j} \sigma_{i j}$. For an orthotropic lamina, the plane stresses in the principle material orientation is defined below by Eq.3.8

$$
\left\{\begin{array}{l}
\sigma_{1} \\
\sigma_{2} \\
\sigma_{3}
\end{array}\right\}=\left[\begin{array}{ccc}
Q_{11} & Q_{12} & 0 \\
Q_{11} & Q_{22} & 0 \\
0 & 0 & Q_{66}
\end{array}\right]\left\{\begin{array}{c}
\epsilon_{1} \\
\epsilon_{2} \\
\gamma_{12}
\end{array}\right\}
$$

In the above equation, $Q_{i j}$ represents the reduced stiffness matrix, where the components of $Q_{i j}$ are defined below in Eq. 3.9, 3.10, 3.11, and 3.12.

$$
\begin{gathered}
Q_{11}=\frac{E_{1}}{1-v_{12} v_{21}} \\
Q_{22}=\frac{E_{2}}{1-v_{12} v_{21}} \\
Q_{12}=\frac{E_{1} v_{21}}{1-v_{12} v_{21}}=\frac{E_{2} v_{12}}{1-v_{12} v_{21}} \\
Q_{66}=G_{12}
\end{gathered}
$$

Using the above Eq 3.9-3.12, The magnitudes of the extension in each respective principle material orientation under normal stress are applied in an orthogonal orientation. An orthotropic 
material has a different response to loading depending on the orientation, and different moduli and Poisson's ratios exist in the principle material orientation.

$$
A_{i j}=\sum_{k=1}^{\infty}\left(\bar{Q}_{i j}\right)_{k}\left(h_{k}-h_{k-1}\right)
$$

$A_{i j}$ in Equation 3.13 represents the extensional stiffness.

$$
2 B_{i j}=\sum_{k=1}^{\infty}\left(\bar{Q}_{i j}\right)_{k}\left(h_{k}^{2}-h^{2}{ }_{k-1}\right)
$$

$B_{i j}$ in Equation 3.14 represents the coupling stiffness, for simplicity the panels in this thesis are close to symmetric and thus, $B_{i j}$ will be assumed to equal to zero.

$$
D_{i j}=\frac{1}{3} \sum_{k=1}^{\infty}\left(\bar{Q}_{i j}\right)_{k}\left(h_{k}^{3}-h_{k-1}^{3}\right)
$$

$D_{i j}$ in Equation 3.15 represents the bending stiffness.

\subsection{Honeycomb Mechanics}

The stiffness and strength properties of the honeycomb core are required to evaluate the failure mechanisms of the honeycomb panel. Poisson's ratio of honeycomb which is required for failure analysis is defined as $v_{13}$ or $v_{23}$ for in-plane Poisson strains due to out-of plane loading in the three orientation. The elastic modulus of honeycomb in the out-of plane three directions is given by the rule of mixtures expression defined as given in Equation 3.16.

$$
\frac{E_{3}}{E_{s}}=\frac{\rho_{c}}{\rho_{s}}
$$

Where subscripts, c and s, represent core as a whole and constituent material making up the core. The failure of honeycomb occurs due to the fracture of the cell walls under a buckling load. Using the rule of mixtures, one can estimate the relevant collapse strength and stress. Common honeycomb is 
created as arrayed hexagonal cells. The prediction of collapse strength, shear strength, and shear moduli can be found using Equations 3.17, 3.18, 3.19, 3.20 below. $^{19}$

$$
\begin{aligned}
\sigma_{c} & =3.25 \sigma_{s}\left(\frac{\rho_{c}}{\rho_{s}}\right)^{5 / 3} \\
\frac{\tau_{31}}{E_{s}} & =1.7\left(\frac{\rho_{c}}{\rho_{s}}\right)^{3} \\
\frac{G_{31}}{G_{s}} & =0.375\left(\frac{\rho_{c}}{\rho_{s}}\right) \\
\frac{G_{32}}{G_{s}} & =0.6\left(\frac{\rho_{c}}{\rho_{s}}\right)
\end{aligned}
$$

Where $E_{3}$ is the compressive Young's modulus for the core and $\rho_{c}$ and $\rho_{s}$ are the density of the core and face sheet, respectively. The anisotropy of the honeycomb leads to a dependence of skin failure loads on the orientation of the honeycomb. Similarly, the shear strength of the core depends on the orientation of the cells within the honeycomb. See Table 5.2 for values produced by Eq. (3.17)$(3.20) .^{19}$

\subsection{Failure Mode Analysis}

Currently, a reliable ultimate failure criterion has only been partially successful. The issue is that sandwich structures generally fail by series of local failures at internal stress concentrations such as loading points or defects which in turn leads to global failure and so must be accounted. The effect of these localized failures for the most part is greater in larger sections, where the face sheets are comparable small when compared to the core. Manufacturing process's play a key role on the structures efficiency, see section 2 . 
Understanding failure modes allow the designer to ensure that no component of their composite sandwich is over-designed when compared to other components and can assist in weight optimization with respect to the static test requirements. Subjecting composite sandwiches to 3-point bending results in multiple types of failures, such as face fracture, face wrinkling, core shear yield, core compressive yield, and interfacial failure between the core and the faces. ${ }^{23}$ The failure mode analysis in this report developed on by the works of Triantafillou and Gibson ${ }^{26}$ will investigate face failure, core compressive yield, and core shear yield, which are the three most typical failures from the experimentation. Sufficient analysis of sandwich structures require a thorough knowledge of the mechanical behavior of the face sheets and the core. The face sheets act in a relatively predictable manner, as modelled by laminated plate theory. The mechanical modelling of the core is not so straight forward, particularly for honeycomb. The addition of core behavior of core to shear loading from the face sheets or loading perpendicular to the face sheet planes is needed. This response is affected by the core relative density which is a ratio of the core density as a whole to the density of the materials making up the core.

Considering a constant core and face sheet thickness and constant face sheet orientation, analysis was then done with varying core angles. This resulted in varying core Young's moduli and core shear yield strength with respect to core angle of the honeycomb. Using this, bending stiffness and flexural rigidity of the beam where then calculated at specific core angles using Eq. $3.4 .^{23}$ The bending stiffness of the face about its individual axis was beneficial in solving for the core's shear and compressive stress and the face's compressive stress as well as the failure loads per unit width due to the three types of failures mentioned earlier. The local bending moment and deflection could also be calculated using this value.

The face compressive stress in the beam is calculated using the equation below: 


$$
\sigma_{f, \text { total }}=\sigma_{f, \text { overall }}+\sigma_{f, \text { local }}=\frac{P L}{4 t_{f} d}+\frac{6 P}{4 t_{f}^{2} \beta}
$$

Where $P$ is the central load per unit width, $L$ is the length of the beam, and $\beta$ is a variable that is defined by $\beta=\sqrt[4]{k / 4 D_{f}}$ with $k=E_{c} / t_{c}$. The $\beta$ term is dependent on the face bending stiffness defined earlier.

To understand how the beam theoretically should fail due to the five main types of failure mentioned earlier, maximum loads per unit width were calculated for each failure condition using the equations below:

$$
\begin{gathered}
P_{\max }=\frac{X_{f}}{\left(\left(L / 4 t_{f} d\right)+\left(6 / 4 t_{f}^{2} \beta\right)\right)} \quad \text { (Face Compressive Failure) } \\
P_{\max }=2 S_{c} d \quad \text { (Core Shear Yield) } \\
P_{\max }=\frac{2 X_{c}}{\beta} \quad \text { (Core Compressive Yield) } \\
P_{\max }=\frac{t_{f}\left(\pi^{2} E_{f} c d\right)^{\frac{1}{3}}}{3 L} \quad \text { (Indentation with Face Elastic Buckling) } \\
P_{\max }=\frac{4 t_{f} d X_{f}}{L} \text { (Micro buckling) }
\end{gathered}
$$

Where $\mathrm{X}_{\mathrm{f}}, \mathrm{S}_{\mathrm{c}}$, and $\mathrm{X}_{\mathrm{c}}$ are the the face compressive strength, core shear yield strength, and core compressive yield strength, respectively. This report investigates the effects of changing core angle, so the Young's modulus of the core and the core shear yield strength will be varied with the varying core angle. Eq. 3.21 above varies with core angle due to the core shear yield strength value and Eq. 3.21-23 vary due to the $\beta$ term, which is dependent on the core Young's modulus value. Micro buckling, is not an 
active constraint, when $\frac{X_{f}}{X_{c}}$ is large or $\mathrm{L}$ is small, in which case Eq 3.22-23 become the active constraints as is the case with the materials in this thesis. Indentation with face elastic buckling only tends to become active when face sheet thickness is much less than core thickness. The inclusion of the indentation failure mode departs from classical theory due to the inclusion of plastic collapse of the core material and a strong interaction of between the face loading and displacement of the core. These five maximum failure loads where plotted against each other, shown in Figure 3.1, to show where the beam will likely fail due to the five failure conditions with respect to core orientation mentioned earlier.

From Figure 3.1, the yielding loads for the three types of failures using experimental mechanical properties can be seen. For a core angle of $0^{\circ}-30^{\circ}$, the sandwich will yield due to core compression and for a core angle of $30^{\circ}-90^{\circ}$, the sandwich will yield due to shearing of the core. According to the plot, the

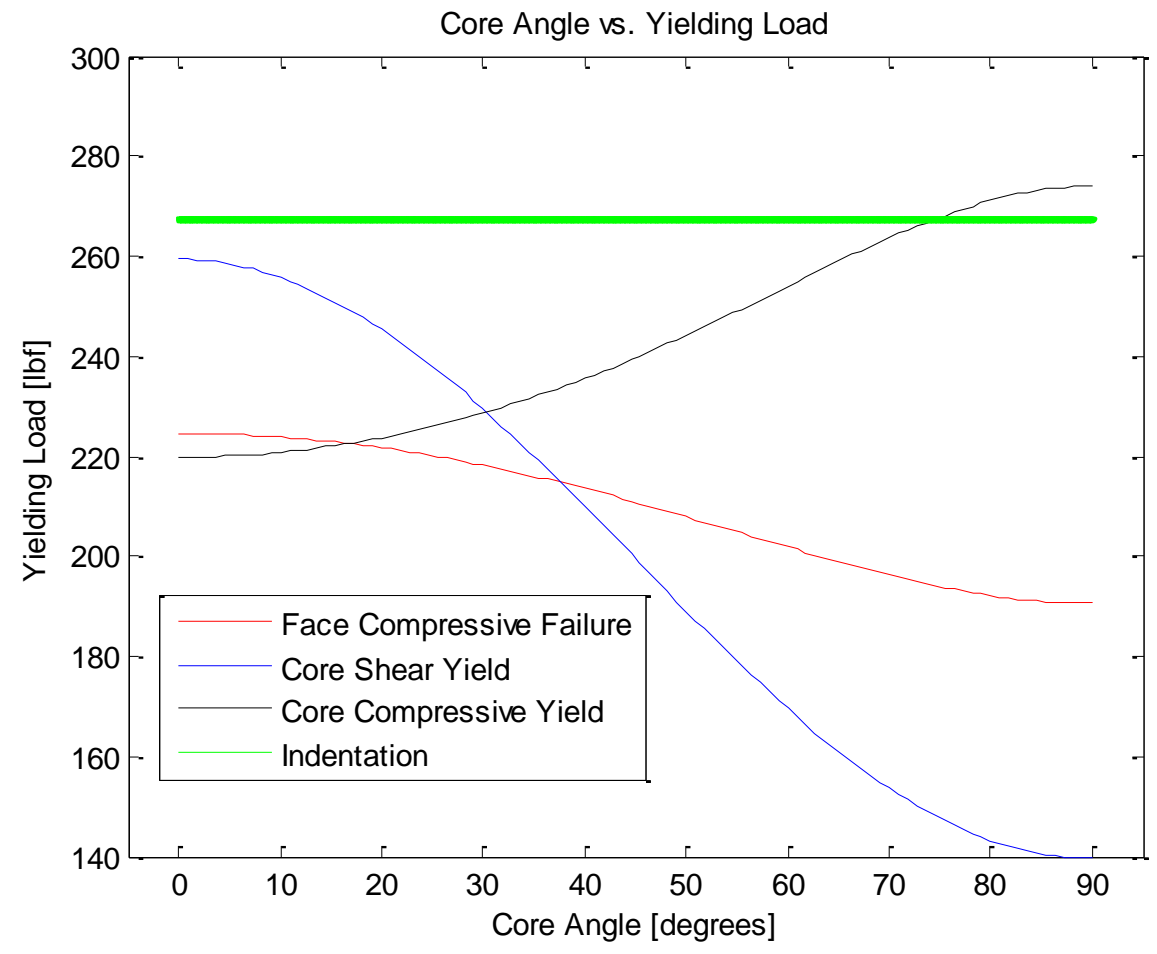

Figure 3.1. Core angle vs. yielding loads for three main types of failure

sandwich will fail due to compression of the face after yielding; however, this does not take into account 
failure of the beam due to core shear or compression because these values were not able to be experimentally gathered for honeycomb.

These failure mode loads can be manipulated to estimate failure loads for specimens with varying face sheet thicknesses, but can be used only as a guide because all the above equations are simplified with the assumption of symmetric panels with face sheet to core thickness ratio's less than 0.1, particularly with the core shear failure mode. The analytical model for failure due to core shear slightly under predicts failure load due to the exclusion of the effects the face sheets have on bending strength, Thus for higher face sheet thickness's $(>0.036)$ core shear estimates become less accurate. For specimens used in this thesis, specimens with a total layer count over 6 layers violates the face sheet to core thickness ratio of less than 0.1 , and thus predicted failure loads should be taken strictly as a guide to estimate which failure mode will occur first. Figure 3.2 shows the failure loads for specimens with 
constant bottom face sheet thickness set at 0.012. All other configuration maps can be viewed in the appendix.

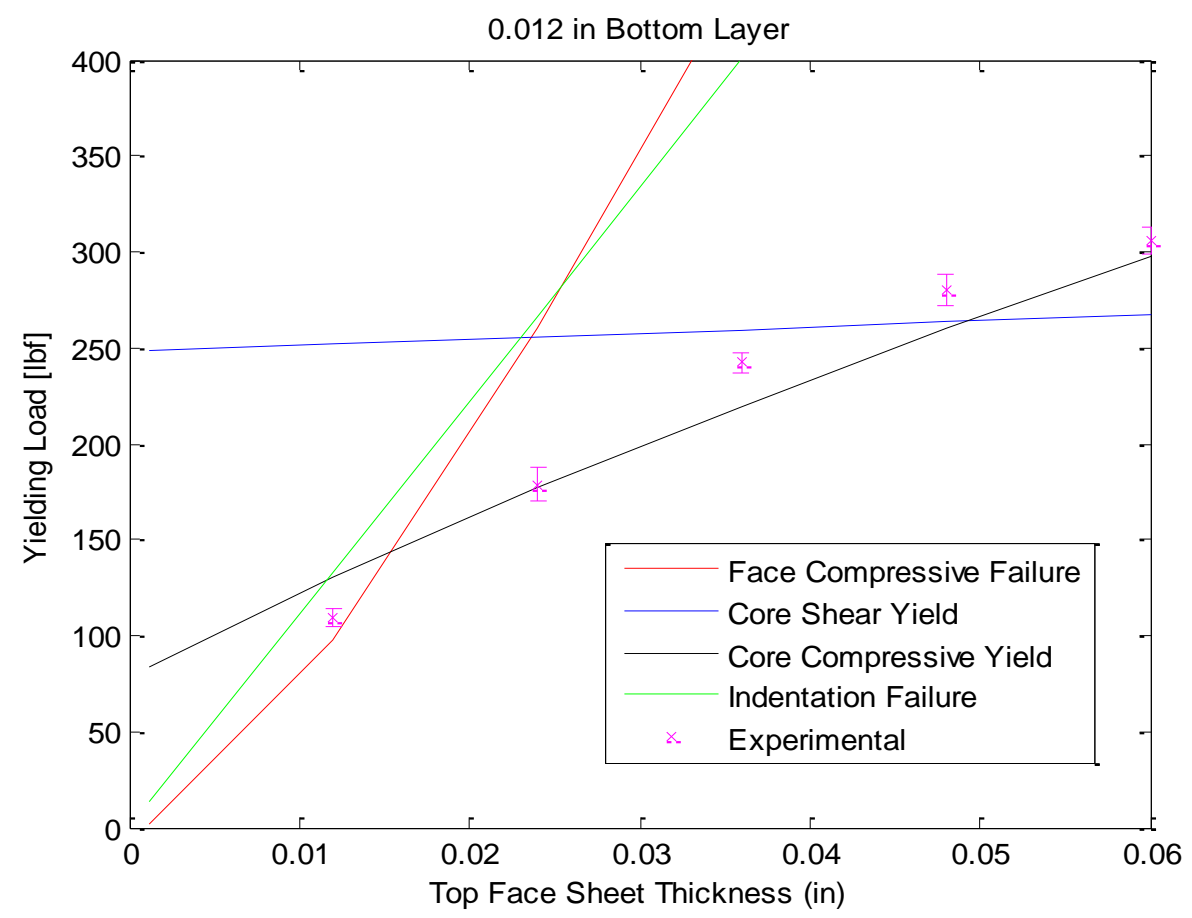

Figure 3.2. Failure Loads for Specimens with Varying Top Face Sheet thickness and Bottom Layer Constant at 0.012 in

The main focus of this analysis, however, was the creation of static failure mode maps. To do this, transition equations between the failure modes had to be used to create a visual of how they interact with each other. The three equations used are shown below:

$$
\begin{gathered}
\frac{L^{*}}{2 s^{*} \sigma^{*} t^{*}}+\frac{3\left(1+t^{*}\right)}{s^{*} \sigma^{*} t^{*}} \sqrt[4]{\frac{E^{*}}{3 t^{*}}}=1 \quad \text { (Face andCore Shear) } \\
\frac{L^{*}}{2 \sigma^{*}\left(1+t^{*}\right)} \sqrt[4]{\frac{E^{*}}{3 t^{*}}}+\frac{1}{\sigma^{*}} \sqrt{\frac{3 E^{*}}{t^{*}}}=1 \quad \text { (Face and Core Compressive) } \\
\frac{s^{*} t^{*}}{\left(1+t^{*}\right)} \sqrt[4]{\frac{E^{*}}{3 t^{*}}}=1 \quad \text { (Core Shear and Core Compressive) }
\end{gathered}
$$


These three equations use non-dimensional parameters that are defined as $t^{*}=t_{f} / t_{c}, L^{*}=$ $L / t_{c}, E^{*}=E_{f} / E_{c}, \sigma^{*}=X_{f} / X_{c}, s^{*}=X_{c} / S_{c}$. Solving these three equations for $t^{*}$ and $\mathrm{L}^{*}$ allowed for a plot

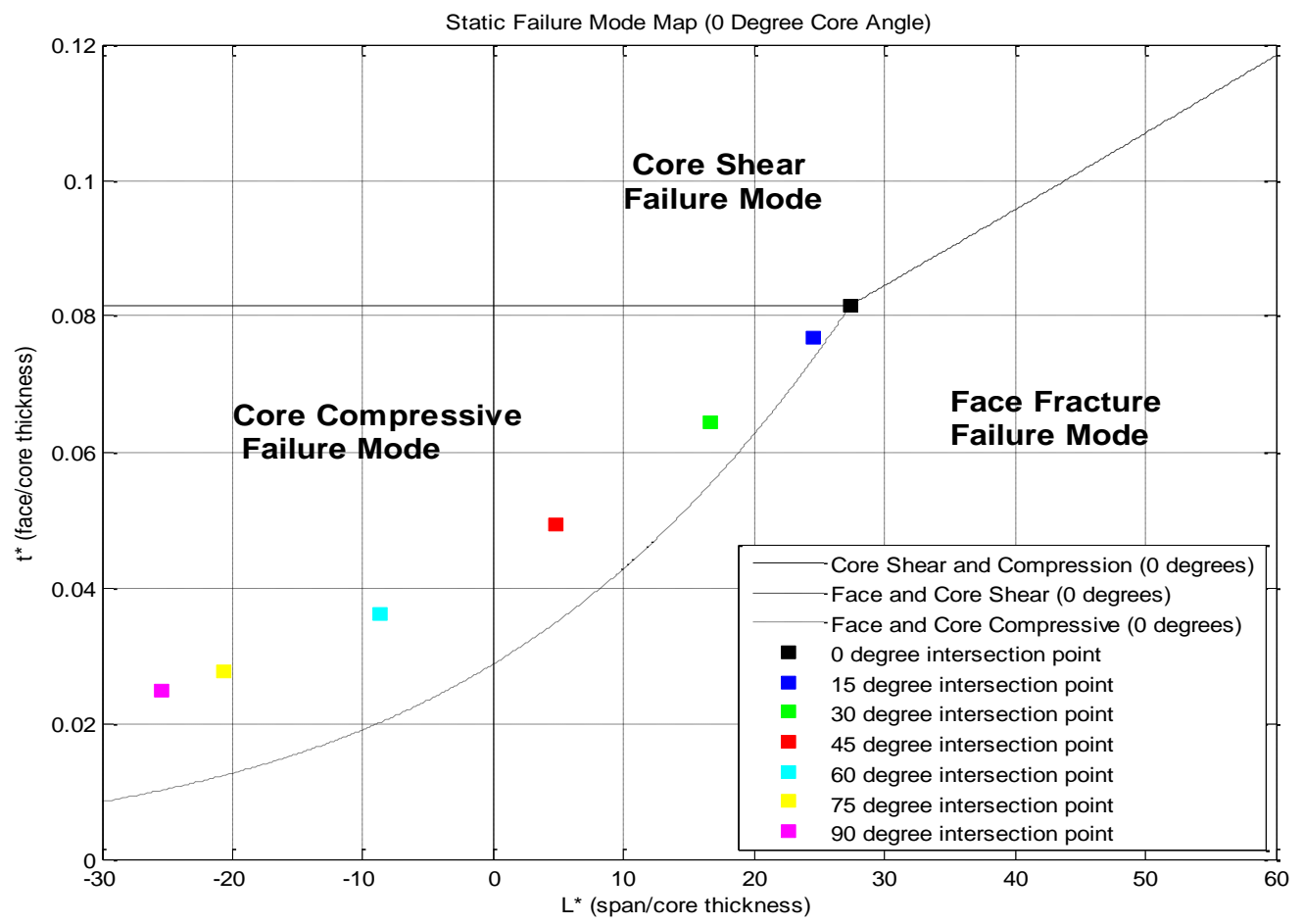

Figure 3.3. Static failure mode map for 0 degree core angle, where $s^{*}=2.154 \mathrm{E}^{*}=350.638 \sigma^{*}=191.428$

of $L^{*}$ vs. $t^{*}$ to be created. Figure 3.3 shows this plot and describes the areas where each failure will occur. It can be seen from the plot that the failure modes occur between the transition lines that account for that certain failure mode. For example, the core compressive failure mode occurs between the core shear and core compressive line and the face and core compressive line. The interaction points show, in general, that as the core changes from $0^{\circ}$ to $90^{\circ}$, the core compressive failure mode decreases while the other modes increase. For example, for a constant span length, the core thickness would have to be increased and/or the face thickness would have to be decreased to create a better likelihood of core compressive failure. A decrease in core thickness would be more likely to result in face fracture failure and an increase in face thickness would be more likely to result in core shear failure. 
Understanding how this plot works allows for optimization of beam design depending on type of failure desired and beam dimensions. Failure mode maps for the other six core angles can be seen in the Appendix section at the end of this report. Our experimental specimens had $t^{*}=0.0640$ and $L^{*}=16$ to ensure failure was due to core compression or shear only. Core shear and compression failure is needed to determine the effect of core ribbon orientation on the composite sandwich. Had experimental parameters been chosen that failure occurred in the face fracture regime; there would be little difference in failure loads.

\subsection{Theoretical Midspan Deflections of Varying Core Ribbon Orientation}

Using Classical beam and plate theory from section 3.1, theoretical midpsan deflections at $100 \mathrm{lbf}$ applied load were calculated for test specimens shown in table 3.1

Table 3.1. Beam and Plate Theory midspan deflections for varying core angle specimens at $100 \mathrm{lbf}$

\begin{tabular}{|c|c|c|c|}
\hline $\begin{array}{c}\text { Core } \\
\text { Orientation }\end{array}$ & $\begin{array}{c}\text { Beam } \\
\text { Theory } \\
\text { Deflection } \\
\text { (in) }\end{array}$ & $\begin{array}{c}\text { Plate } \\
\text { Theory } \\
\text { Deflection } \\
\text { (in) }\end{array}$ & $\begin{array}{c}\text { Difference } \\
\text { (\%) }\end{array}$ \\
\hline $\mathbf{0}$ & 0.03517 & 0.0350 & 0.403 \\
\hline $\mathbf{1 5}$ & 0.035927 & 0.0358 & 0.316 \\
\hline $\mathbf{3 0}$ & 0.038264 & 0.0382 & 0.169 \\
\hline $\mathbf{4 5}$ & 0.042303 & 0.0423 & 0.074 \\
\hline $\mathbf{6 0}$ & 0.047805 & 0.0478 & 0.031 \\
\hline $\mathbf{7 5}$ & .0532900 & 0.0533 & 0.015 \\
\hline $\mathbf{9 0}$ & 0.055746 & 0.0577 & 0.012 \\
\hline
\end{tabular}

As can be seen these specimens have little difference between beam and plate theory. The specimens' width is comparatively small compared to its length and so falls under the beam theory regime. 


\subsection{Theoretical Midspan Deflections of Varying Face Sheet Thickness's}

Using Classical beam and plate theory from section 3.1, theoretical midpsan deflections at $100 \mathrm{lbf}$ applied load were calculated for test specimens shown in tables 3.2-4.

Table 3.2. Beam Theory midspan deflections for varying Face sheet thickness specimens at $100 \mathrm{lbf}$

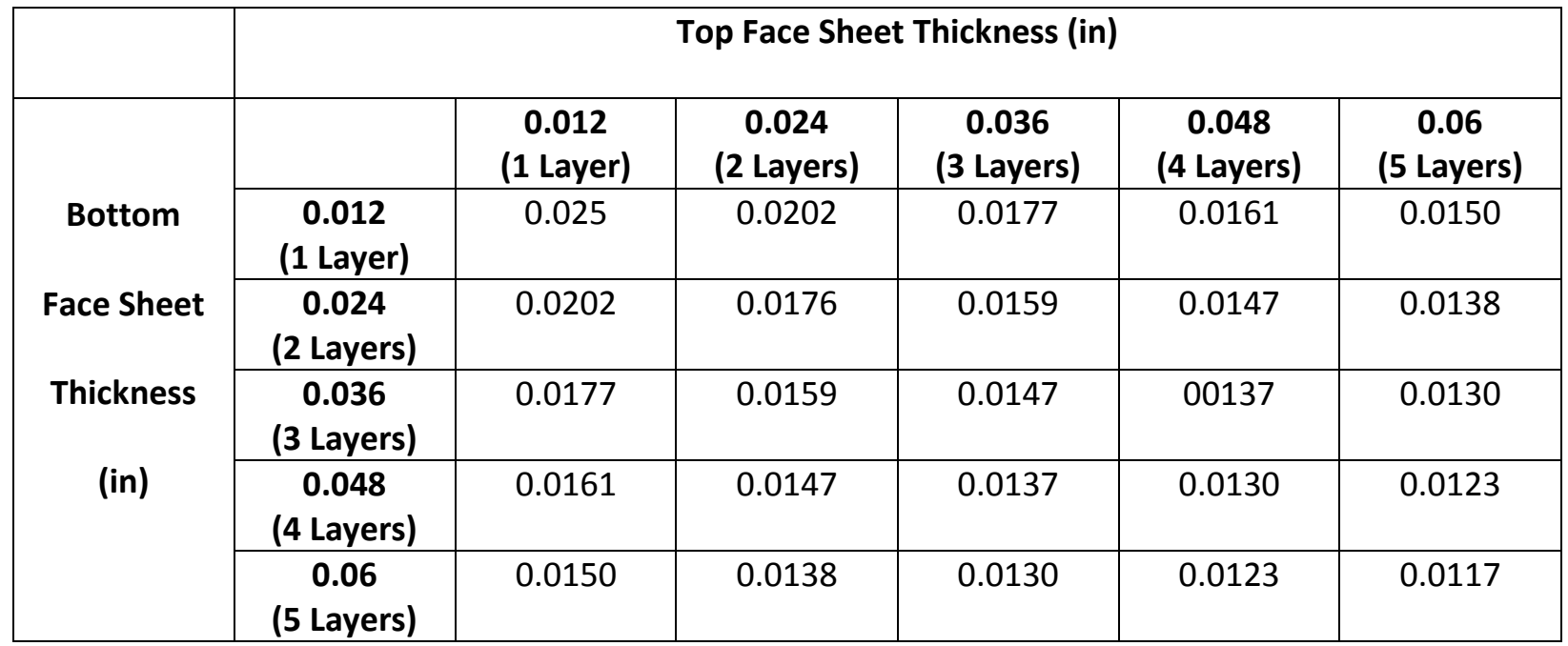

Table 3.3 Plate Theory deflections for varying Face sheet thickness specimens at $100 \mathrm{lbf}$

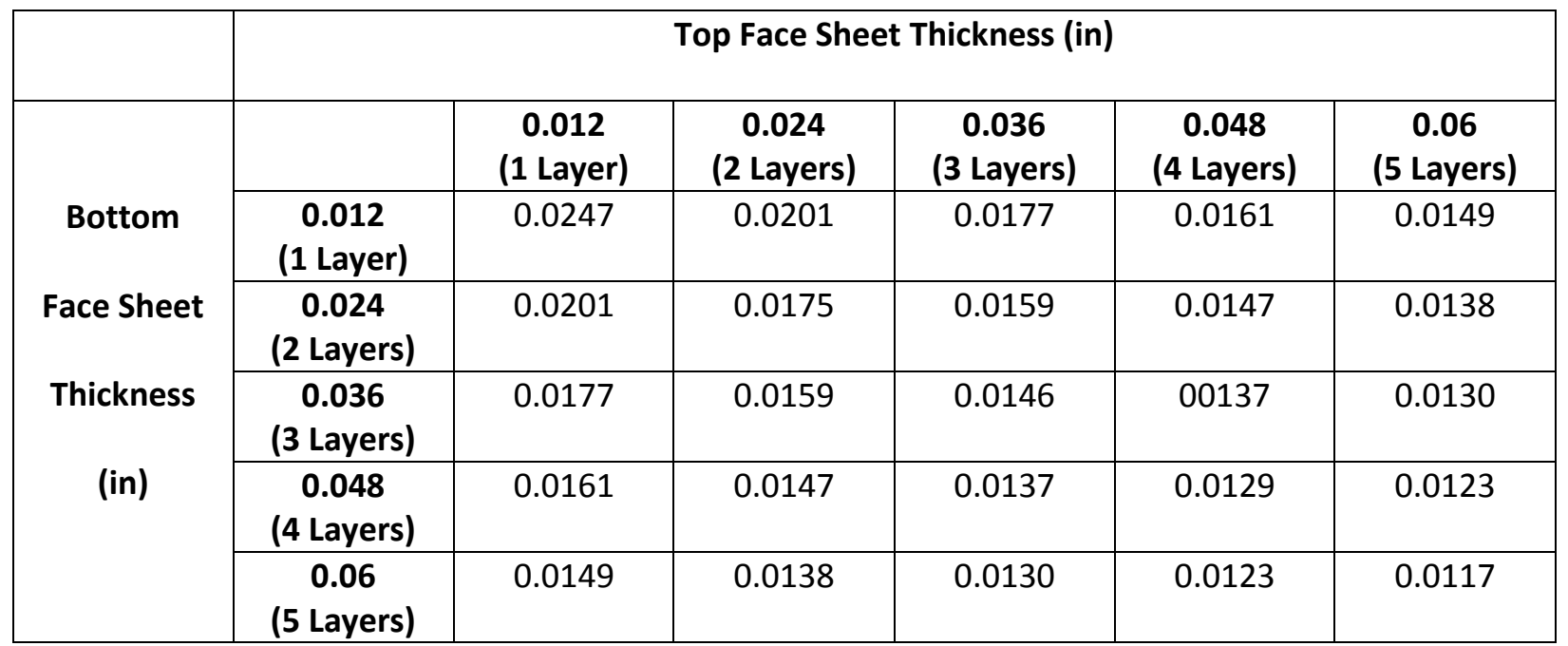


Table 3.4 Differences between Beam and Plate Theory midspan deflections for varying Face sheet thickness specimens at $100 \mathrm{lbf}$

\begin{tabular}{|c|c|c|c|c|c|c|}
\hline & \multicolumn{6}{|c|}{ Top Face Sheet Thickness (in) } \\
\hline & & $\begin{array}{c}0.012 \\
\text { (1 Layer) }\end{array}$ & $\begin{array}{c}0.024 \\
\text { (2 Layers) }\end{array}$ & $\begin{array}{c}0.036 \\
\text { (3 Layers) }\end{array}$ & $\begin{array}{c}0.048 \\
\text { (4 Layers) }\end{array}$ & $\begin{array}{c}0.06 \\
\text { (5 Layers) }\end{array}$ \\
\hline Bottom & $\begin{array}{c}0.012 \\
\text { (1 Layer) }\end{array}$ & $1.24 \%$ & $0.66 \%$ & $0.42 \%$ & $0.29 \%$ & $0.22 \%$ \\
\hline Face Sheet & $\begin{array}{c}0.024 \\
\text { (2 Layers) }\end{array}$ & $0.66 \%$ & $0.40 \%$ & $0.27 \%$ & $0.20 \%$ & $0.15 \%$ \\
\hline Thickness & $\begin{array}{c}0.036 \\
\text { (3 Layers) }\end{array}$ & $0.42 \%$ & $0.27 \%$ & $0.19 \%$ & $0.14 \%$ & $0.11 \%$ \\
\hline \multirow[t]{2}{*}{ (in) } & $\begin{array}{c}0.048 \\
\text { (4 Layers) }\end{array}$ & $0.29 \%$ & $0.19 \%$ & $0.14 \%$ & $0.11 \%$ & $0.09 \%$ \\
\hline & $\begin{array}{c}0.06 \\
\text { (5 Layers) }\end{array}$ & $0.21 \%$ & $0.15 \%$ & $0.11 \%$ & $0.09 \%$ & $0.07 \%$ \\
\hline
\end{tabular}

Again we can see that there is little difference between theoretical beam and plate theory

midpsan deflections. The specimens used for varying face sheet thickness were double the width than the specimens manufactured for varying core orientation giving a width to span ratio of 0.5 . As the width increases the structure moves away from being classified as a beam and closer into the plate regime. The Plate theory deflections shall be used for later comparisons with experimental and numerical data.

\subsection{Bending Stiffness and Strength Optimization}

Sandwich construction involves mating high modulus face sheets with thick, and relatively light core. Through design maximum stiffness and strength can be obtained through minimum weight combinations. Optimization of face sheet to core thickness ratios are crucial in minimizing cost in design. Design optimization of sandwich structures with respect to weight is presented by Froud. ${ }^{47}$ Froud optimizes face sheet to core weight analytically for maximizing bending stiffness and bending strength. Johnson and $\mathrm{Sims}^{48}$ reviewed the method for optimum design with respect to minimum weight, improving the design of sandwich construction. 


\subsubsection{Bending Stiffness}

By using the equation for flexural rigidity, derived from equations 3.9-12, the main contribution to bending resistance is the middle term and may be written as:

$$
D=E_{f}\left(t_{b}+t_{t}\right)\left(\frac{\left(d+\frac{t_{b}+t_{t}}{2}\right)}{2}\right)^{2}
$$

As well as the combined weight of the face sheets and core given by:

$$
W=\rho_{c} d+t_{t} \rho_{f}+t_{b} \rho_{f}
$$

Where $\rho_{c}$ and $\rho_{f}$ are the densities of the core and face sheet materials respectively.

$$
\text { If } \varphi=\frac{\rho_{c} d}{W} \text { and }(1-\varphi)=\frac{\rho_{f}\left(t_{t}+t_{b}\right)}{W}
$$

$t_{t}, t_{b}$ and $c$ can be expressed in terms of $\varphi$. The maximum flexural rigidity will occur when

$$
\frac{d D}{d \varphi}=0
$$

Solving for $\varphi$ the minimum and maximum values are obtained.

$$
\begin{gathered}
\varphi_{\text {minimum }}=\frac{\rho_{c}}{\rho_{c}-2 \rho_{f}} \\
\varphi_{\text {maximum }}=\frac{3 \rho_{c}-4 \rho_{f}}{3 \rho_{c}-6 \rho_{f}}
\end{gathered}
$$

For the materials used in this thesis,

$$
\begin{gathered}
\varphi_{\text {minimum }}=0 \\
\varphi_{\text {maximum }}=0.658
\end{gathered}
$$


Which means that the minimum flexural rigidity occurs when there is no core present, which makes sense and the maximum theoretical flexural rigidity occurs when

$$
\frac{\rho_{c} d}{\rho_{c} d+2 \rho_{f} t}=0.658
$$

Substituting the material values for this thesis,

$$
\text { total face thickness }=0.04 \text { in }=3.33 \text { layers }
$$

For a symmetrical sandwich panel this would mean a sandwich structure with 1.665 layers on the top and bottom face sheet. Due to geometric constraint on the carbon fiber, this is not possible. Thus the optimum sandwich configuration lies somewhere between the $2 / 1$ and $2 / 2$ configuration. If the stiffness is divided by the weight and then normalized to the minimum value, the optimum stiffness to weight sandwich structure can be obtained.

\subsubsection{Bending Strength}

Using Eq. 3.21-25, theoretical maximum loads for all failure modes can be determined, for all face structure combinations. By dividing the minimum predicted failure load by the structures weight and applying geometric constraints being a minimum of 1 layer for top and bottom face sheets, and a maximum of 6 layers for top and bottom face sheets, the optimal bending strength with respect to minimum weight can be determined shown figure 3.4 


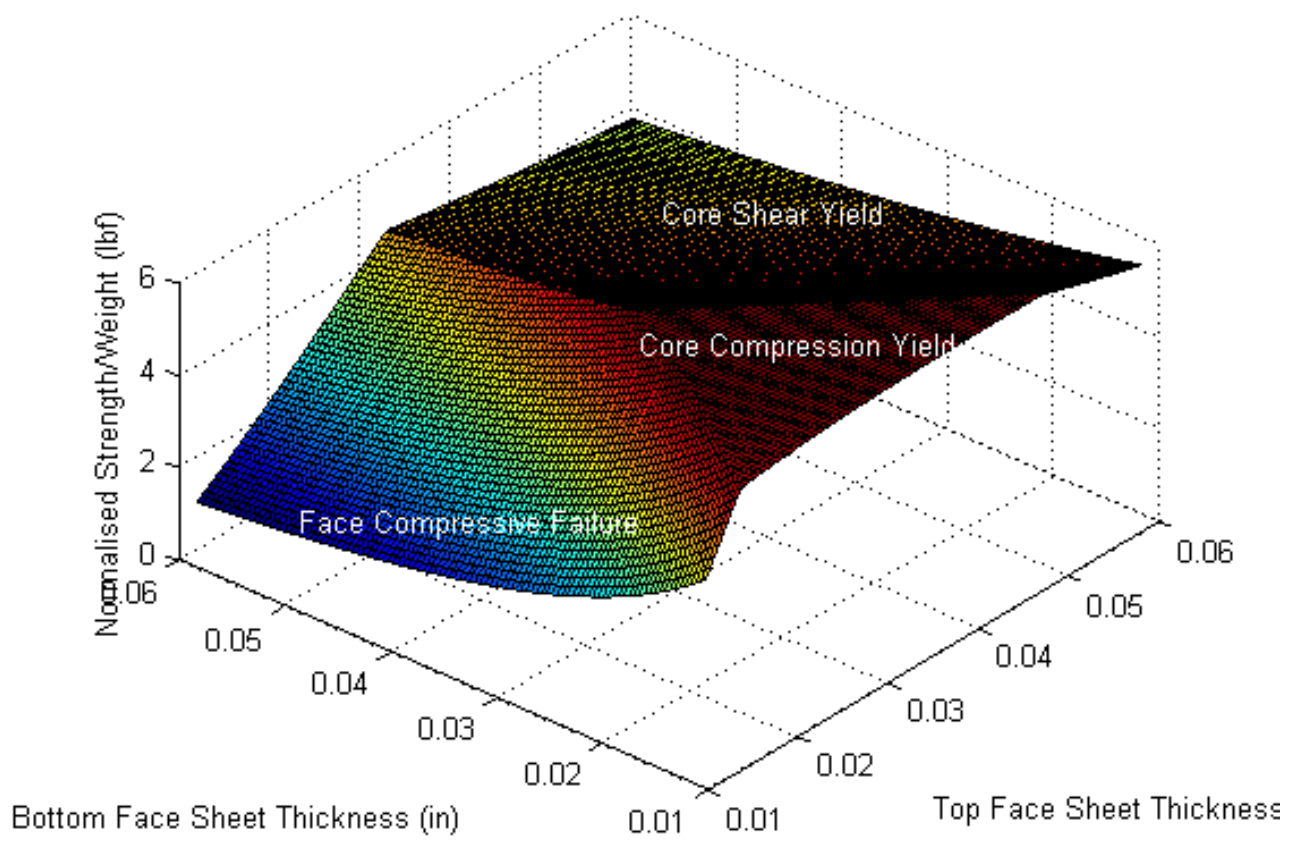

Figure 3.4. Normalized strength to weight ratio with respect to top and bottom face sheet thickness.

The theoretical total face sheet thickness for optimal bending stiffness with respect to minimum weight was calculated to be 0.05698 in (4.741 layers). Examples of calculations can be viewed in the MATLAB code section of the appendix. 


\subsection{Experimental Results, Analysis and Discussion}

This section presents the results of the experimental tests of static 3-point bending specimens.

For each series of tests, the average value, standard deviation and coefficient of variation, \%, were calculated for ultimate strength:

$$
\begin{aligned}
& \bar{x}=\frac{\sum_{i-1}^{n} X_{i}}{n} \\
& S_{n-1}=\sqrt{\frac{\sum_{i-1}^{n} x_{i}^{2}-n \overline{x^{2}}}{n-1}} \\
& C V=100 * \frac{S_{n-1}}{x}
\end{aligned}
$$

Where $\mathrm{x}$ is sample mean (average), $S_{n-1}$ is the sample standard deviation, $C V$ is the sample coefficient of variation, $\%, n$ is the number of tested specimens, and $X_{i}$ is the measured or derived property.

\subsection{Toe Compensation}

In a typical force versus displacement curve (see Figure. 4.1) there is a toe region, AC, which does not represent a property of the material. It is caused by the take up of slack and alignment of the test

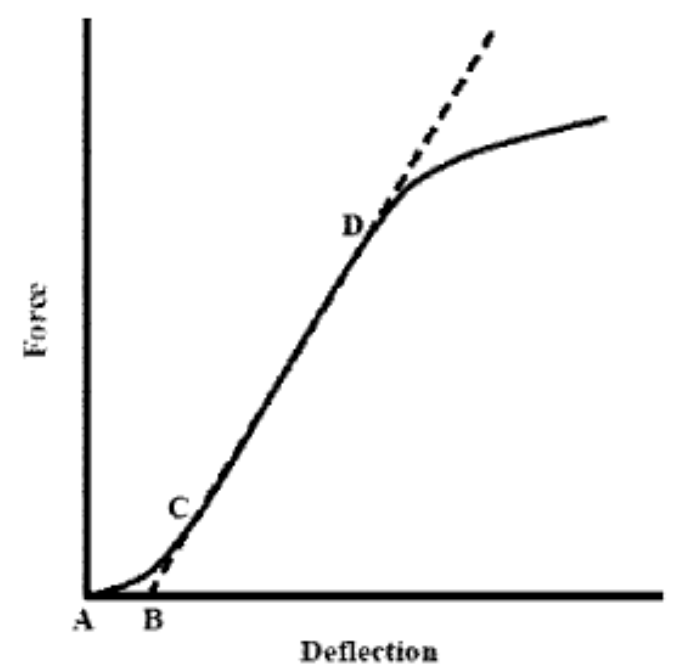

Figure 4.1. Sandwich Material with Hookean Response Region

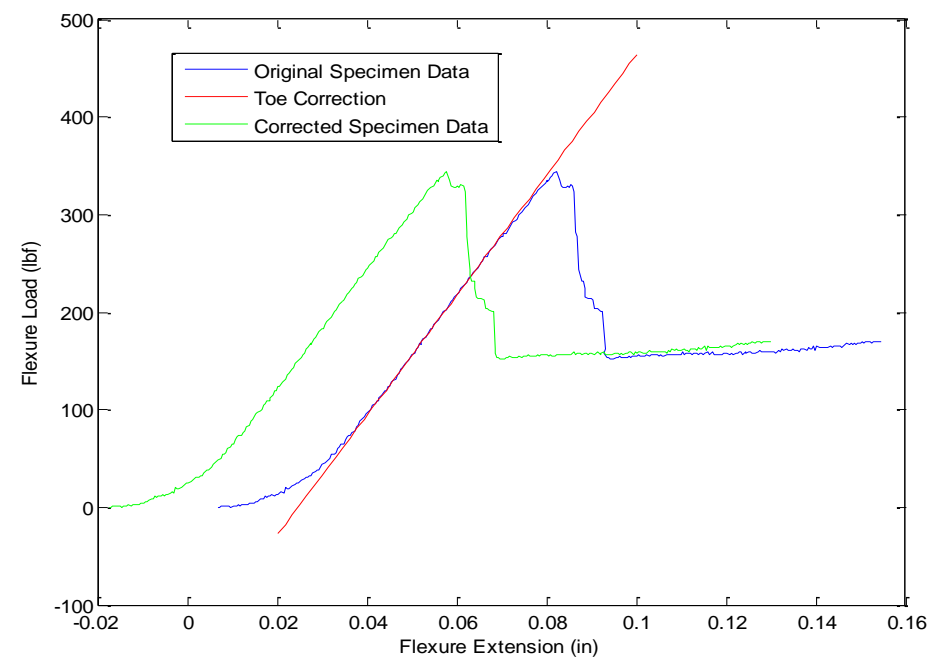

Figure 4.2. Toe correction Example 
specimen. To extract correct data, the AC portion must be compensated to get the correct zero displacement point. By continuing the linear Hookean region $(C D)$ of the curve will give the correct zero displacement point (B) where $\delta=0.0$ and all displacements are then measured from that point (B).

\subsection{Experimental Analysis of sandwiches with varying core orientation}

For Sandwich structure optimization, it is critical to determine the effects of varying the honeycomb core orientation. Varying core orientation effects ultimate core strength, sandwich stiffness, sandwich ultimate load at failure, and sandwich extension at failure. For the purposes of this paper, the longitudinal orientation of the honeycomb shall be considered 0 degrees orientation and the transverse orientation being 90 degrees of orientation. Table 3.1 shows how varying the orientation of the honeycomb within the composite sandwich affects the structural properties of the sandwich when subjected to 3-point bending.

Table 4.1. Effects of core angle on composite sandwich structural properties

\begin{tabular}{|c|c|c|c|c|c|c|}
\hline $\begin{array}{c}\text { Core } \\
\text { Orientation }\end{array}$ & $\begin{array}{l}\text { Ultimate } \\
\text { Load } \\
\text { (Ibf) }\end{array}$ & $\begin{array}{c}\text { D } \\
\left(\mathrm{lbf} / \mathrm{in}^{3}\right)\end{array}$ & $\begin{array}{c}\mathrm{U} \\
\text { (lbf) }\end{array}$ & $\begin{array}{c}\text { G } \\
\left(\text { (lbf/in }{ }^{2}\right)\end{array}$ & $\begin{array}{l}\text { Extension } \\
\text { at failure } \\
\text { (in) }\end{array}$ & $\begin{array}{c}\text { Core Shear Ultimate } \\
\text { Stress (psi) }\end{array}$ \\
\hline 0 & $\begin{array}{l}301.61 \\
\pm 5.051\end{array}$ & $3.725 \mathrm{e} 4$ & $6.49 \mathrm{e} 3$ & $10.2 \mathrm{e} 3$ & $\begin{array}{c}0.1016 \\
\pm 0.0281\end{array}$ & $262.27 \pm 4.392$ \\
\hline 15 & $\begin{array}{r}274.9 \\
\pm 10.00\end{array}$ & $3.7243 \mathrm{e} 4$ & $6.29 \mathrm{e} 3$ & $9.87 e 3$ & $\begin{array}{c}0.1007 \\
\pm 0.0032\end{array}$ & $237.80 \pm 8.65$ \\
\hline 30 & $\begin{array}{c}264.71 \\
\pm 5.77\end{array}$ & $3.7213 \mathrm{e} 4$ & $5.73 \mathrm{e} 3$ & $9 e 3$ & $\begin{array}{c}0.1042 \\
\pm 0.000286\end{array}$ & $228.86 \pm 4.984$ \\
\hline 45 & $\begin{array}{c}248.16 \\
\pm 9.09\end{array}$ & $3.7176 \mathrm{e} 4$ & $4.96 \mathrm{e} 3$ & $7.8 \mathrm{e} 3$ & $\begin{array}{c}0.1170 \\
\pm 0.00052\end{array}$ & $214.67 \pm 7.867$ \\
\hline 60 & $\begin{array}{c}246.02 \\
\pm 4.29\end{array}$ & $3.7142 \mathrm{e} 4$ & $4.2 \mathrm{e} 3$ & $6.6 \mathrm{e} 3$ & $\begin{array}{c}0.1336 \\
\pm 0.000517\end{array}$ & $213.09 \pm 3.72$ \\
\hline
\end{tabular}




\begin{tabular}{|c|c|c|c|c|c|c|}
\hline $\mathbf{7 5}$ & $\begin{array}{c}238.13 \\
\pm 5.10\end{array}$ & $3.7119 \mathrm{e} 4$ & $3.64 \mathrm{e} 3$ & $5.72 \mathrm{e} 3$ & $\begin{array}{c}0.1425 \\
\pm 0.000213\end{array}$ & $207.19 \pm 4.44$ \\
\hline $\mathbf{9 0}$ & $\begin{array}{c}240.73 \\
\pm 9.23\end{array}$ & $3.7112 \mathrm{e} 4$ & $3.44 \mathrm{e} 3$ & $5.4 \mathrm{e} 3$ & $\begin{array}{c}0.1450 \\
\pm 0.000269\end{array}$ & $205.93 \pm 7.897$ \\
\hline
\end{tabular}

The figures produced below present the core orientation on the horizontal axis while the vertical axis is varying from the maximum extension at failure, maximum facing stress, and maximum force. Each figure's data was found to represent a sinusoidal curve varying over the orientation of the core.

In Figure 4.3, the left vertical axis shows the Failure Load with right vertical axis showing the Failure Extension. The maximum extension at a given load occurs when the core is in the transverse position which is defined as $90^{\circ}$ from the longitudinal core ribbon orientation.

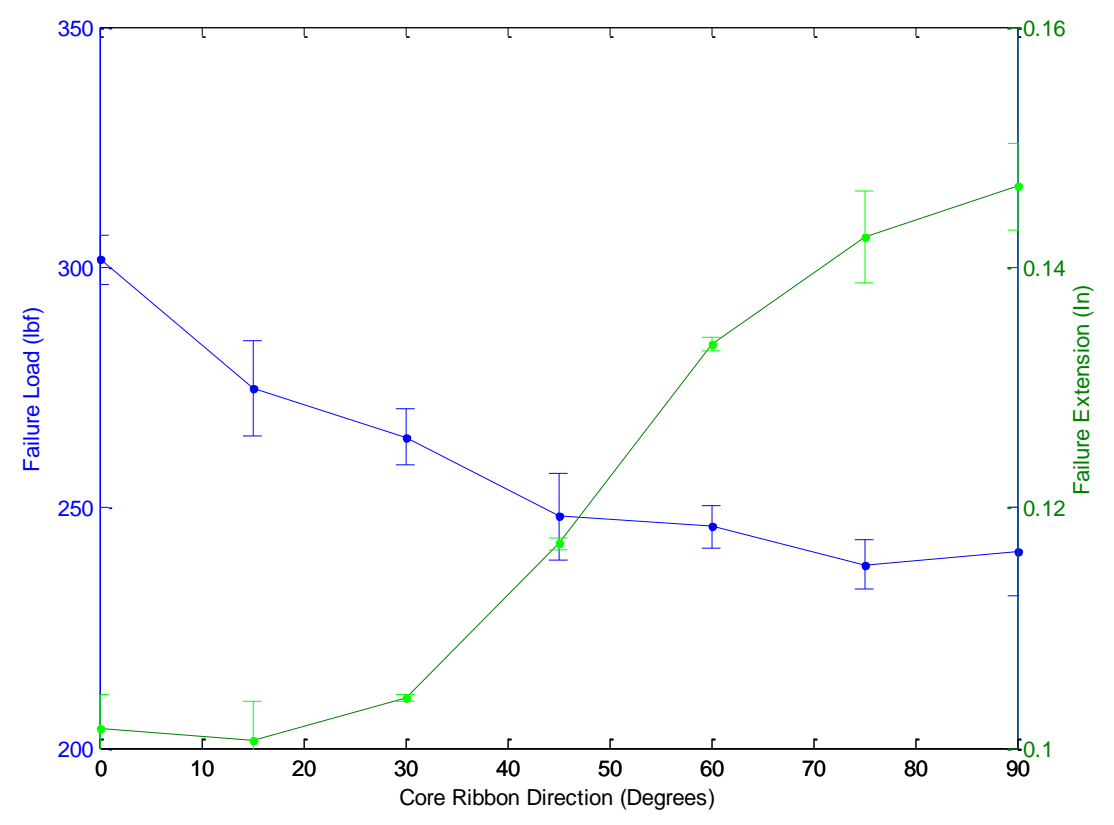

Figure 4.3. Failure Load and Extension vs core orientation

The maximum force that the specimen can withhold before failure occurs at approximately 300 Ibf when the core is in the longitudinal orientation. Similar to the maximum facing stress plot, the trend follows a sinusoidal curve. The minimum force occurs at the transverse position of $90^{\circ}$, which is 
approximately $240 \mathrm{lbf}$. The reason for this variation in maximum load with core ribbon value is due to the fact that the longitudinal core ribbon orientation has a much higher Young's modulus value than the transverse orientation. Failure, again, is occurring in the core so this variation in the longitudinal and transverse Young's moduli directly correlate to the variation in failure load values. This lower value of force indicates that depending on the application, the ideal core orientation for minimal extension and higher loading is the longitudinal $0^{\circ}$ orientation. If more extension is desired in the application with lower stress, then the transverse core orientation is ideal. The most compliant core ribbon orientation is the transverse orientation, at $90^{\circ}$, where the max extension value in inches is approximately 0.145 . Whereas, the stiffest ribbon orientation is at $0^{\circ}$, or the longitudinal orientation, where the smallest maximum extension occurs and is approximately 0.1 inches. This lower value of extension shows that the longitudinal orientation of core has a higher flexural rigidity than the transverse orientation.

In Figure 4.4, the vertical axis represents the maximum facing stress of the specimens at their failure loads. These values were calculated from Eq. 3.2, which is a function strictly of the load. The maximum facing stress occurs when the core is in the longitudinal orientation and the facing stresses vary with core angle, which shows that the failure is occurring in the core. If failure was to occur within the face sheets, the maximum facing stress values would be roughly the same for varying core angles due to the fact that the structure of the faces does not change between specimens. 


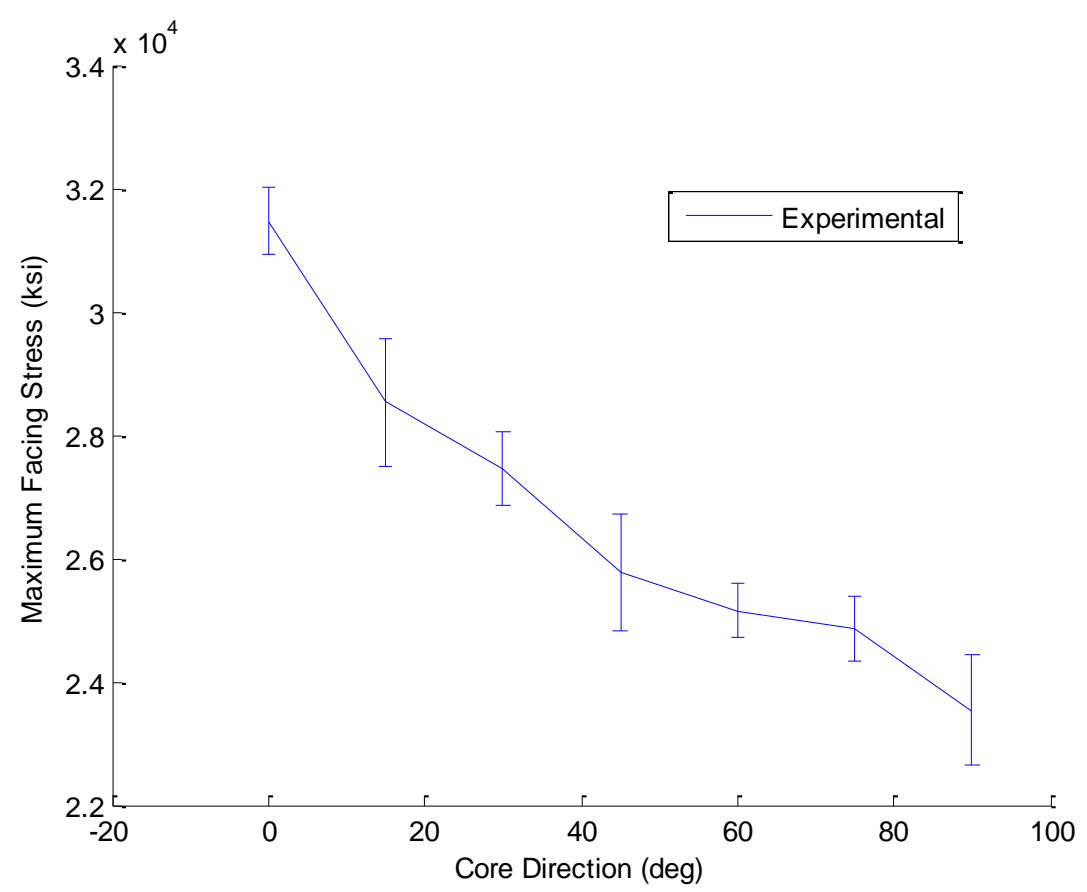

Figure 4.4. Theoretical maximum facing stress at failure vs. core orientation

Figure 4.5 shows the normalized stiffness with respect to core ribbon orientation.

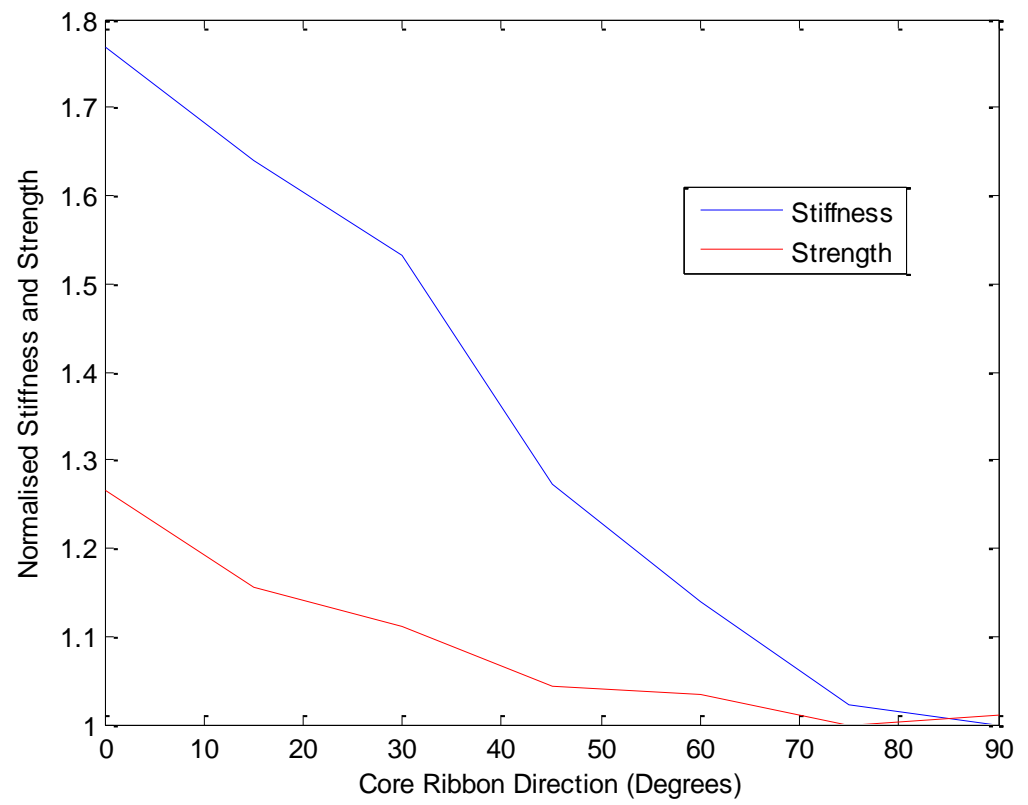

Figure 4.5. Normalised Stiffness and Strength with respect to Core Ribbon Orientation 
As can be seen the $0^{\circ}$ degree core ribbon orientation specimen produced the stiffest behavior with approximately $80 \%$ increase in stiffness from the $90^{\circ}$ degree core ribbon orientation. This is due to stiffness being a function of Young's moduli and the longitudinal Young's moduli of the honeycomb is twice that of its transverse orientation. The bending strength of the specimen decreased with the increase of core direction.

Table 4.2. Theoretical and Experimental mid-span Deflection comparison with respect to Core Ribbon Orientation at 100lb Load

\begin{tabular}{|l|l|l|l|}
\hline $\begin{array}{l}\text { Core Ribbon } \\
\text { Orientation } \\
\text { (Degrees) }\end{array}$ & $\begin{array}{l}\text { Theoretical Deflection } \\
\text { at 100lb (in) }\end{array}$ & $\begin{array}{l}\text { Experimental } \\
\text { Deflection at 100lb (in) }\end{array}$ & Error (\%) \\
\hline 0 & 0.03517 & $0.0337 \pm 0.00078$ & $4.179 \%$ \\
\hline 15 & 0.03592 & $0.0363 \pm 0.0011$ & $1.058 \%$ \\
\hline 30 & 0.03826 & $0.038 \pm 0.0012$ & $0.679 \%$ \\
\hline 45 & 0.0423 & $0.0453 \pm 0.0013$ & $7.09 \%$ \\
\hline 60 & 0.04781 & $0.0486 \pm 9.13 \mathrm{e}-4$ & $1.652 \%$ \\
\hline 75 & 0.05329 & $0.0555 \pm 0.0022$ & $3.771 \%$ \\
\hline 90 & 0.05575 & $0.0558 \pm 0.0019$ & $0.089 \%$ \\
\hline
\end{tabular}

Using a deflection equation that takes into account shear rigidity and bending stiffness values, theoretical deflection values were calculated that compare quite well to experimental values. Typically the deflection due to shear rigidity is significantly lower than the deflection due to bending stiffness so it is often neglected; however, when the core's Young's moduli is significantly lower than face Young's moduli, the deflection due to shear rigidity cannot be neglected. The shear rigidity decreased with a decrease in core Young's moduli, which resulted in an increase in the deflection due to shear rigidity as can be seen with the deflection increase from $0^{\circ}-90^{\circ}$ core ribbon angle. The slight difference in deflection 
values between theoretical and experimental could be due to multiple variables throughout the experiment such as layup quality, material quality, the assumption of 0.012 in per layer varying with layup thickness and/or discrepancies in tolerances within the 3-point bend machine. Fiber volumes for the carbon fiber face sheets were very close to theoretical values, which tells us that the material's resin content is still intact and probably not much of a factor in the deflection error. Overall, however, the low error percentages give numerical proof of a well-designed and executed 3-point bend experiment of the composite sandwiches.

\subsection{Experimental Analysis of sandwiches with varying face sheet thicknesses}

Three inch wide Specimens with top and bottom face sheet thickness's ranging from 0.012 in to 0.06 in were tested under a three point bend test with a 6 in support span. Deflections and extensions can be seen in the below tables and figures below.

Table 4.3 Experimental Failure Loads for varying Face sheet thickness specimens (Ibf)

\begin{tabular}{|c|c|c|c|c|c|c|}
\hline & \multicolumn{7}{|c|}{ Top Face Sheet Thickness (in) } \\
\hline \multirow{4}{*}{ Bottom } & & $\mathbf{0 . 0 1 2}$ & $\mathbf{0 . 0 2 4}$ & $\mathbf{0 . 0 3 6}$ & $\mathbf{0 . 0 4 8}$ & $\mathbf{0 . 0 6}$ \\
& & $\mathbf{1}$ Layer) & (2 Layers) & (3 Layers) & (4 Layers) & (5 Layers) \\
\cline { 2 - 7 } & $\mathbf{0 . 0 1 2}$ & 328.11 & 536.49 & 726.65 & 840.122 & 917.07 \\
& $\mathbf{( 1 ~ L a y e r ) ~}$ & \pm 14.74 & \pm 26.64 & \pm 17.08 & \pm 23.72 & \pm 21.12 \\
\cline { 2 - 7 } Face Sheet & $\mathbf{0 . 0 2 4}$ & 312.82 & 556.10 & 730.85 & 960.04 & 1050.4 \\
& $\mathbf{( 2 ~ L a y e r s )}$ & \pm 15.48 & \pm 20.72 & \pm 7.23 & \pm 23.43 & \pm 13.01 \\
\cline { 2 - 7 } Thickness & $\mathbf{0 . 0 3 6}$ & 308.69 & 530.77 & 747.91 & 934.67 & 1154.7 \\
& $\mathbf{( 3 ~ L a y e r s )}$ & \pm 8.65 & \pm 8.34 & \pm 11.07 & \pm 25.8 & \pm 27.68 \\
\cline { 2 - 7 } (in) & $\mathbf{0 . 0 4 8}$ & 326.69 & 510.00 & 750.15 & 987.41 & 1134.1 \\
& $\mathbf{( 4 ~ L a y e r s )}$ & \pm 18.78 & \pm 9.91 & \pm 29.07 & \pm 58.06 & \pm 31.36 \\
\cline { 2 - 7 } & $\mathbf{0 . 0 6}$ & 337.72 & 533.69 & 835.97 & 995.21 & 1166.0 \\
& $\mathbf{( 5 ~ L a y e r s )}$ & \pm 8.18 & \pm 18.39 & \pm 25.71 & \pm 30.99 & \pm 43.24 \\
\hline
\end{tabular}

Figure 4.6 shows that adding layers on the bottom face sheet has little to no increase in failure load unless the top face sheet to bottom face sheet ratio was greater than two as indicated by constant top layer counts 4 and 5. Constant top layer count 1, 2 and to an extent 3 show no increase failure load indicating that the top face sheet yield load exceeds bottom face sheet yield load. Figure 4.7 shows the 
effect of increasing the top face sheet thickness. A three dimensional representation of the failure loads for all specimen configurations shows the overall behavior of all specimens, with little increase in failure load along constant top face sheet lines and roughly $200 \mathrm{lbf}$ increment increases along constant bottom face sheet lines. Adding a second layer to the top face sheet increases failure load by approximately $64 \%$. Adding a third layer increases failure load by $68 \%$ from the two layer specimens. When a fourth layer is added the average failure load increase is slightly less at $56 \%$. A fifth layer results in a smaller increase yet at $43 \%$. Figure 4.7 shows that increasing the top to bottom face sheet ratio past 2.5 results in less of an increase in failure load from previous increments.

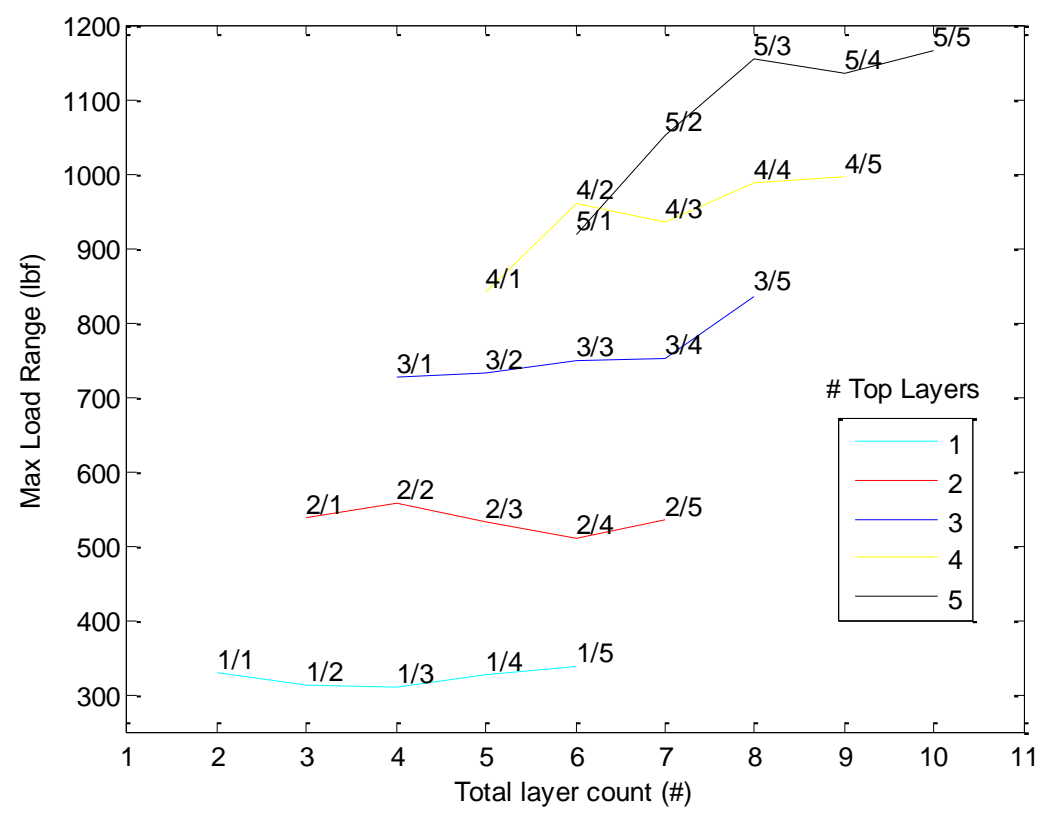

Figure 4.6. Failure Load for varying face sheet thickness, Constant top layer. \#/\# indicates Top face layers/Bottom face layers 


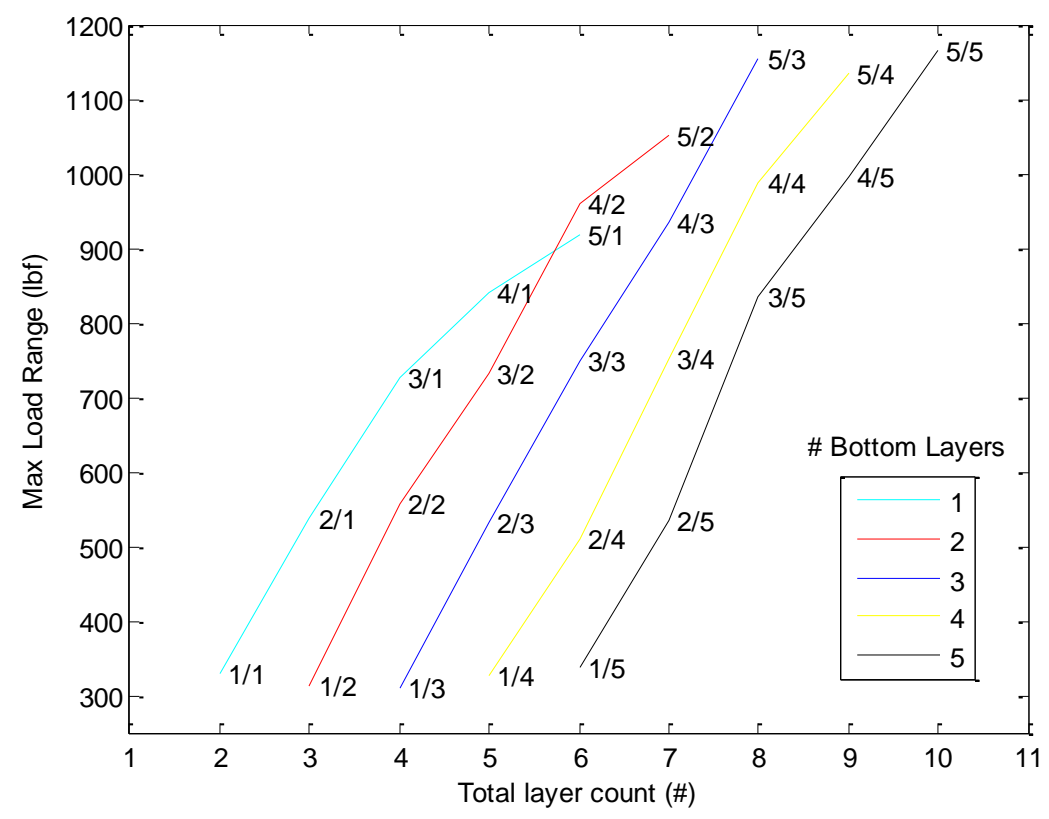

Figure 4.7. Failure Load for varying Face Sheet Thickness, Constant Bottom Layer

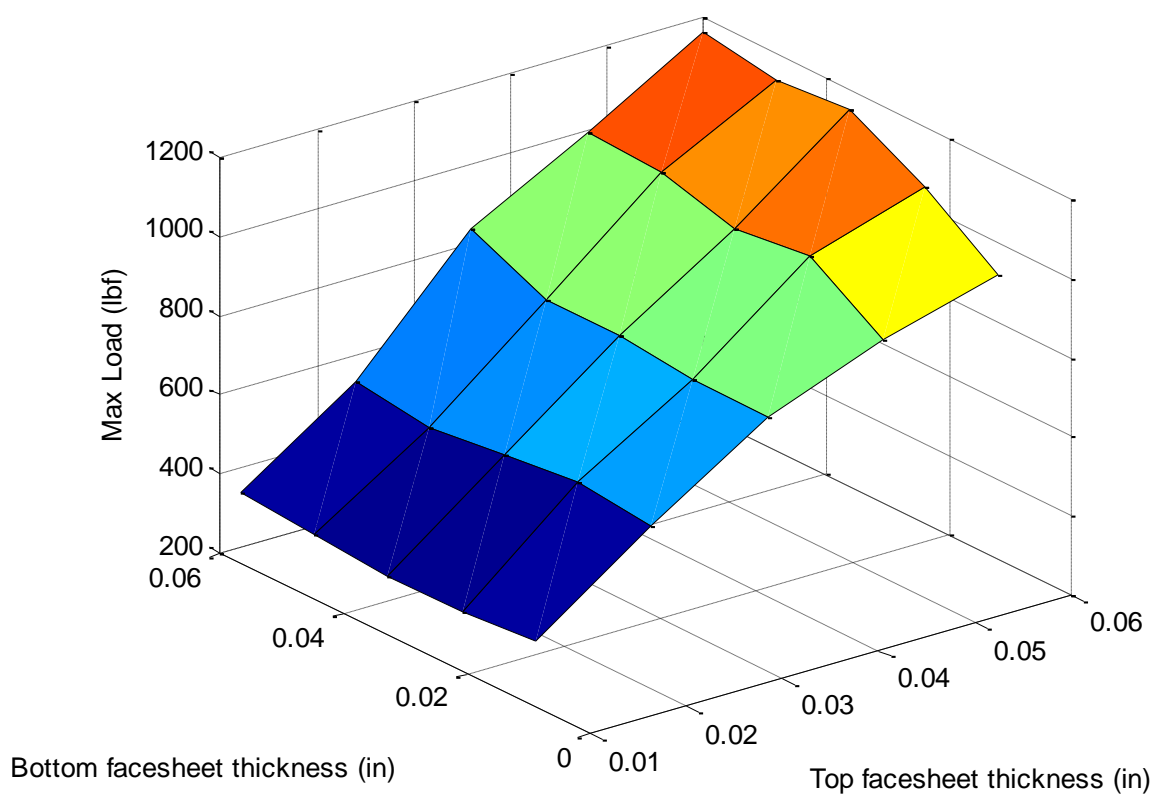

Figure 4.8. Three Dimensional Perspective of Failure Loads for varying Face Sheet Thickness's 
Table 4.4 and Figures show the experimental midspan failure extensions. The specimen with 0.012 in top face sheet and 0.06 in bottom face sheet had the minimum failure extension, but with a comparatively large weight and low failure load, it was the least efficient sandwich structure. Increasing bottom face sheet thickness along constant top face sheet lines, decreases maximum failure extension by approximately $30 \%$, but with increased weight. Increasing top face sheet thickness increases Failure extension by approximately $100 \%$ from 0.012 to 0.06 along constant bottom face sheet thickness lines. Figure 4.9 shows a three dimensional perspective on the overall effects on failure extension for all specimen configurations.

Table 4.4 Experimental Midspan Failure Extensions varying Face sheet thickness (in)

\begin{tabular}{|c|c|c|c|c|c|c|}
\hline & \multicolumn{7}{|c|}{ Top Face Sheet Thickness (in) } \\
\hline \multirow{4}{*}{ Bottom } & & $\begin{array}{c}\mathbf{0 . 0 1 2} \\
\mathbf{1} \text { Layer) }\end{array}$ & $\begin{array}{c}\mathbf{0 . 0 2 4} \\
\text { (2 Layers) }\end{array}$ & $\begin{array}{c}\mathbf{0 . 0 3 6} \\
\text { (3 Layers) }\end{array}$ & $\begin{array}{c}\mathbf{0 . 0 4 8} \\
\text { (4 Layers) }\end{array}$ & $\begin{array}{c}\mathbf{0 . 0 6} \\
\text { (5 Layers) }\end{array}$ \\
\cline { 2 - 7 } Face Sheet & $\mathbf{0 . 0 1 2}$ & 0.0873 & 0.1081 & 0.1462 & 0.1611 & 0.1644 \\
& $\mathbf{( 1}$ Layer) & $\pm 4.7 \mathrm{e}-4$ & $\pm 0.1 \mathrm{e}-3$ & $\pm 3.6 \mathrm{e}-4$ & $\pm 1.7 \mathrm{e}-3$ & $\pm 8.7 \mathrm{e}-4$ \\
\cline { 2 - 7 } Thickness & $\mathbf{0 . 0 2 4}$ & 0.0715 & 0.0928 & 0.1124 & 0.1313 & 0.1419 \\
& $\mathbf{( 2 ~ L a y e r s )}$ & $\pm 3.9 \mathrm{e}-4$ & $\pm 5.5 \mathrm{e}-4$ & $\pm 6.2 \mathrm{e}-4$ & $\pm 73 . \mathrm{e}-4$ & $\pm 6.9 \mathrm{e}-4$ \\
\cline { 2 - 7 } & $\mathbf{0 . 0 3 6}$ & 0.0628 & 0.0804 & 0.1054 & 0.1232 & 0.1436 \\
& (3 Layers) & $\pm 5.9 \mathrm{e}-4$ & $\pm 2.3 \mathrm{e}-4$ & $\pm 3.4 \mathrm{e}-4$ & $\pm 7.4 \mathrm{e}-4$ & $\pm 6.2 \mathrm{e}-4$ \\
\cline { 2 - 7 } (in) & $\mathbf{0 . 0 4 8}$ & 0.06061 & 0.070 & 0.098 & 0.1151 & 0.1296 \\
& $\mathbf{( 4 ~ L a y e r s )}$ & $\pm 3.3 \mathrm{e}-4$ & $\pm 2.1 \mathrm{e}-4$ & $\pm 4.7 \mathrm{e}-4$ & $\pm 6.0 \mathrm{e}-4$ & $\pm 5.5 \mathrm{e}-4$ \\
\cline { 2 - 7 } & $\mathbf{0 . 0 6}$ & 0.0606 & 0.701 & 0.0927 & 0.1093 & 0.1251 \\
& $\mathbf{( 5 ~ L a y e r s )}$ & $\pm 1.7 \mathrm{e}-4$ & $3.8 \mathrm{e}-4$ & $\pm 6.5 \mathrm{e}-4$ & $\pm 3.6 \mathrm{e}-4$ & $\pm 3.9 \mathrm{e}-4$ \\
\hline
\end{tabular}

Figure 4.10 shows normalized bending stiffness with respect to minimum weight. Each specimens normalized stiffness value can by divided by its weight to obtain a stiffness to weight ratio, of which each specimen configuration can then be compared. The specimen configurations of $2 / 2$ and $2 / 1$ had the highest stiffness to weight ratio, which agrees with the predicted highest bending stiffness to minimum weight ratio of total layer count 3.33, which lies between the two experimental data points. 


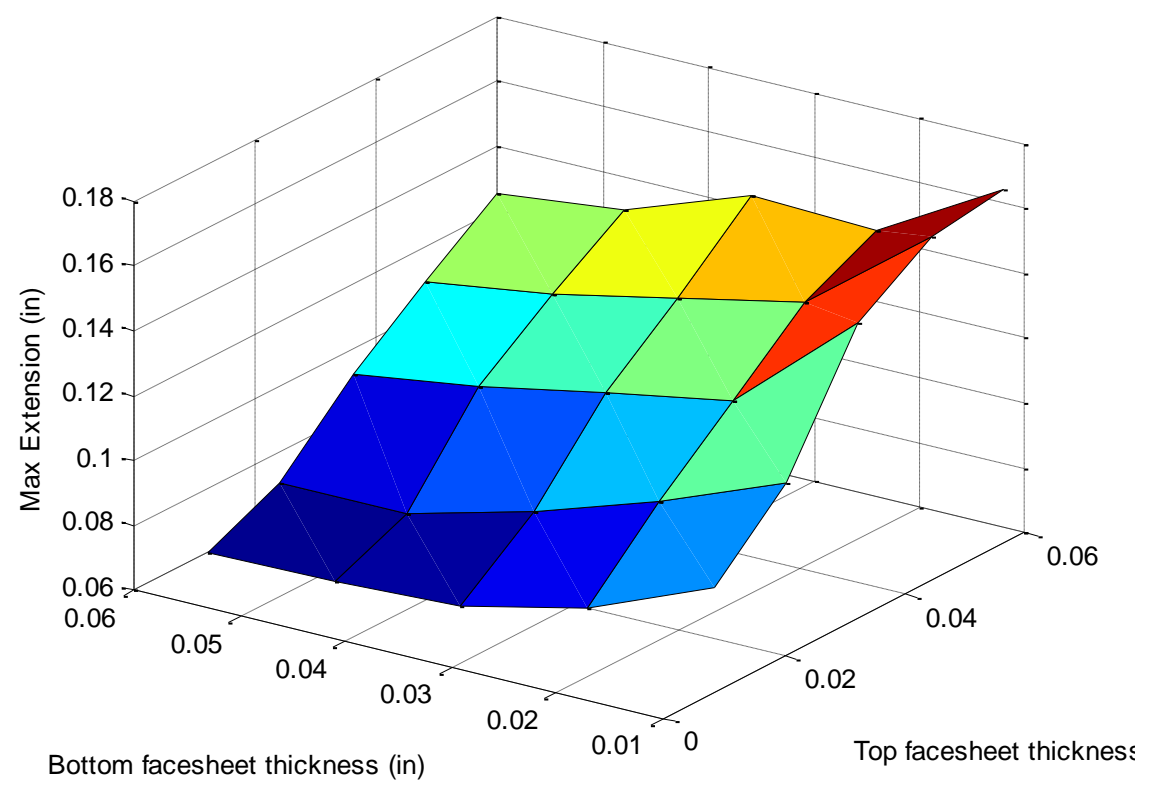

Figure 4.9. Three Dimensional Perspective of Failure Extensions for varying Face Sheet Thickness's

Figure 4.11 depicts the normalized bending strength with respect to minimum weight. The highest bending strength to minimum weight was encountered in the $3 / 1$ (4 total plies) and $4 / 1$ ( 5 total plies) configurations which agrees well with the theoretically determined optimal total ply count of 4.741 plies. It can be seen that increasing bottom face sheet ply count only hinders bending strength with respect to minimum weight. it can be seen that by moving one layer from the bottom face sheet to the top face sheet, a failure load increase of approximately $60 \%$ can be observed for specimens with a total face sheet to core thickness ratio of less than 0.2 , Adding layers after that threshold does little to increase maximum failure load. 


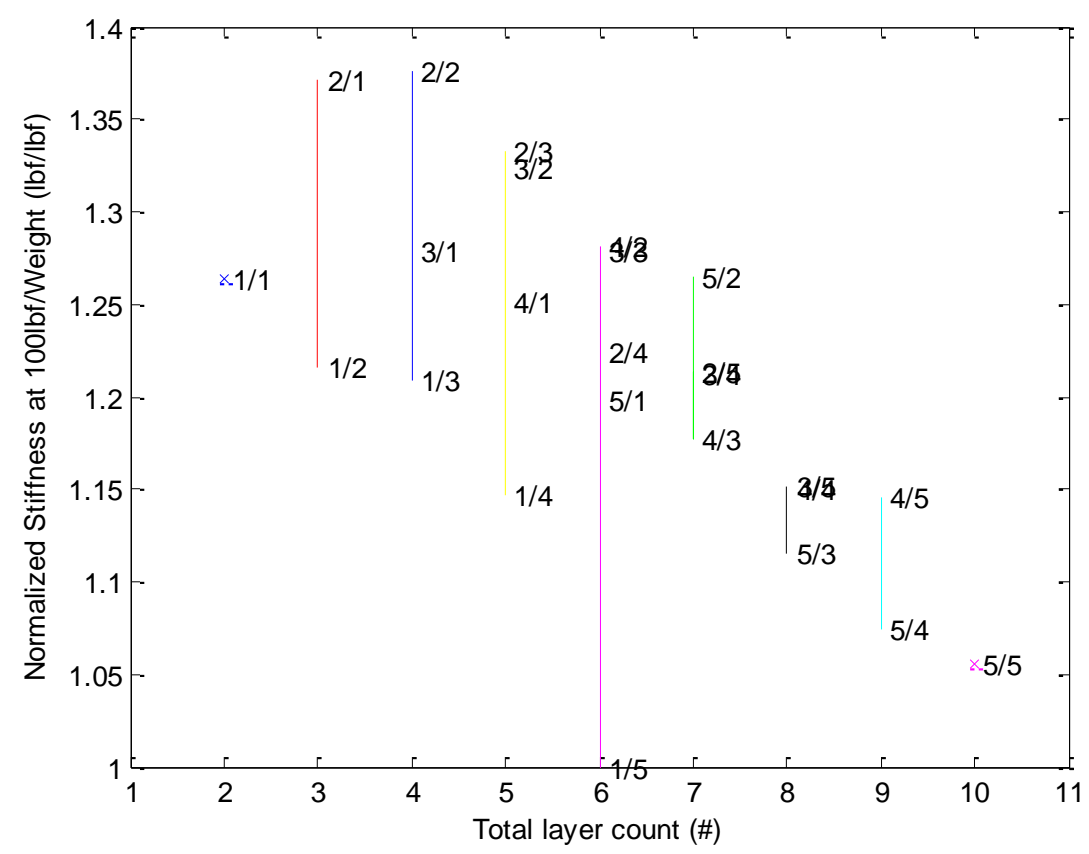

Figure 4.10. Normalized Stiffness by Weight for Varying Face Sheet Thickness \#/\# indicates Top face layers/Bottom face layers

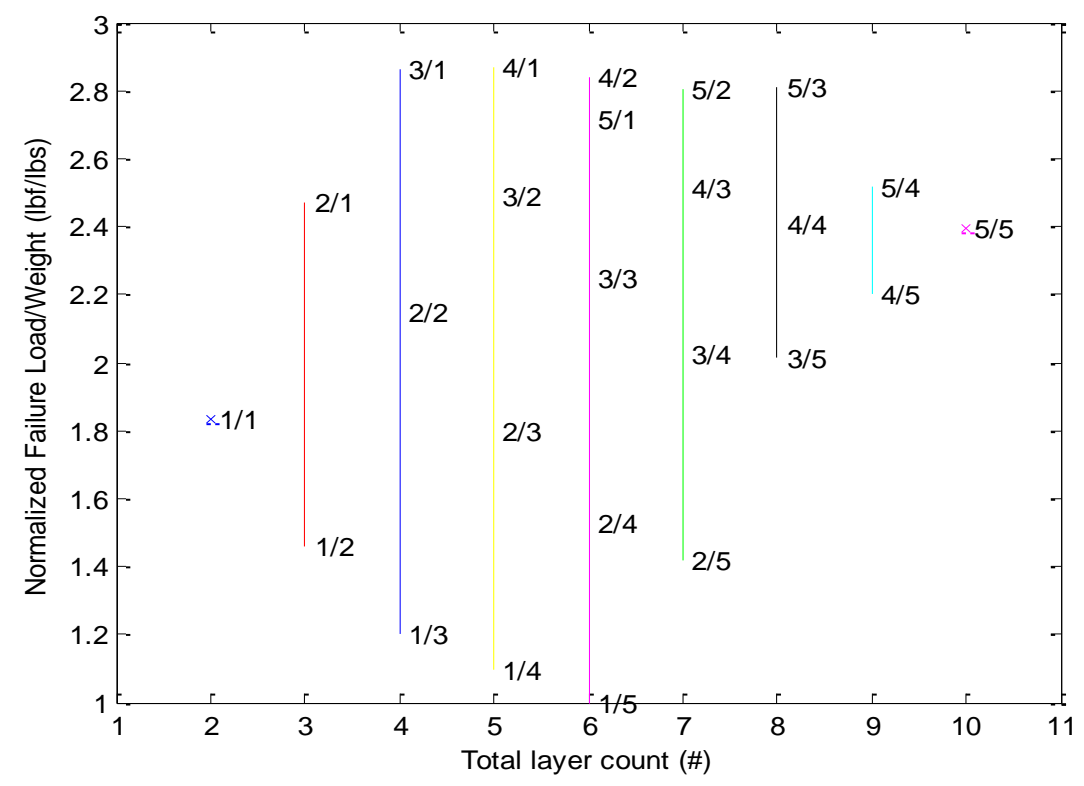

Figure 4.11. Normalized Bending Strength with respect to minimum weight. \#/\# indicates Top face layers/Bottom face layers 


\subsection{FEA Analysis and Results}

This section discusses the process to modelling and analyzing the experimentally tested specimens utilizing numerical methods. Finite element analysis has become the prevalent method for analysis of the behaviors of solids, structures and fluid mechanics. Composite materials and structures can be modelled and successfully analyzed in finite element programs.

Finite element analysis (FEA) was performed on the test composite sandwich beam configurations consisting of composite face sheets and honeycomb core to observe the mechanical behaviors of a simply supported composite sandwich plate under static bending tests. In each case the core orientation was adjusted in $15^{\circ}$ increments from $0^{\circ}$ to $90^{\circ}$ to see the effects of core ribbon orientation on the mechanical properties of the composite sandwich as a whole. Implicit finite element analysis was conducted to numerically analyze the bend tests of the test specimens. Equation 5.1 is the governing equation for implicit analysis.

$$
F=\delta K
$$

The finite element code assumes a displacement function which then the stiffness of the structure $(\mathrm{K})$ is solved and resulting forces are then found. The forces are then used in Equation 5.1 to calculate displacements and then are repeated. Implicit methods have the process re-iterated a number of times until a specified tolerance is met between iterations. Each beam was subjected to three point bending with a $100 \mathrm{lb}$ force acting on across the 1.5 in width of the upper face of the composite structure. The face sheets were modeled as shell structures whilst the core was modeled as a solid element. A convergence study was performed and a seed size of 0.1 was used for analysis. It was found that the deflection of the structure at $100 \mathrm{lb}$ increased from core orientation of $0^{\circ}$ to $90^{\circ}$ in a sinusoidal manner with good agreement with theoretical results. 


\subsection{Development of FEA Model}

The program used in this paper is ABAQUS, which is a program by Dassault Systems. All models were modeled in ABAQUS under three point bending. Each case consists of two structure parts modeled in ABAQUS

- Honeycomb Core

- Composite Face sheets

Each part was defined with an elastic definition, density, and dimension as shown in Table 5.1. All dimensions and numbers were defined in English units. The Mechanical properties shown were found through experimental works conducted under relevant ASTM standards shown in the previous section.

Table 5.1. Mechanical and Part properties of 6367 A280-5H used in ABAQUS Model

\begin{tabular}{|c|c|c|c|}
\hline Mechanical Property & Value & Mechanical Property & Value \\
\hline$\rho$ & $1.436 \mathrm{e}-4 \mathrm{lbf}-\mathrm{s}^{2} / \mathrm{in}^{4}$ & $\mathrm{G}_{12}$ & $6.23 \mathrm{e} 5 \mathrm{psi}$ \\
\hline $\mathrm{E}_{11}$ & $12.936 \mathrm{e} 6 \mathrm{psi}$ & $\mathrm{X}_{\mathrm{C}}=\mathrm{Y}_{\mathrm{C}}$ & $127.7 \mathrm{psi}$ \\
\hline $\mathrm{E}_{22}$ & $12.936 \mathrm{e} 6 \mathrm{psi}$ & $\mathrm{X}_{\mathrm{T}}=\mathrm{Y}_{\mathrm{T}}$ & $168.4 \mathrm{psi}$ \\
\hline $\mathrm{V}_{12}$ & 0.027 & Length & $6 \mathrm{in}$ \\
\hline Part Type & 3-D Shell & Width & $1.5-3 \mathrm{in}$ \\
\hline Section Type & 3-D Shell & Depth & $0.012-0.06$ in \\
\hline
\end{tabular}


Table 5.2. Mechanical and Part properties of HRH-10-1/8-5 Honeycomb used in ABAQUS Model

\begin{tabular}{|c|c|c|c|}
\hline Mechanical Property & Value & Mechanical Property & Value \\
\hline$\rho$ & 7.5e-6 lbf-s ${ }^{2} / \mathrm{in}^{4}$ & $\mathrm{G}_{12}$ & 10200 psi \\
\hline$E_{11}$ & $3.6793 \mathrm{e} 4 \mathrm{psi}$ & $\mathrm{G}_{21}$ & 5400 psi \\
\hline$E_{22}$ & $1.5242 \mathrm{e} 4 \mathrm{psi}$ & $\mathrm{G}_{13}$ & $14151.15 \mathrm{psi}$ \\
\hline$E_{33}$ & 3.7e4 psi & $\mathrm{G}_{23}$ & 8536.73 \\
\hline$V_{12}$ & 0.8036 & $\mathrm{Z}_{\mathrm{c}}$ & 700 psi \\
\hline $\mathrm{V}_{21}$ & 0.4114 & $\tau_{12}$ yield & $325 \mathrm{psi}$ \\
\hline$V_{23}=V_{13}$ & 0.3 & $\tau_{21}$ yield & $175 \mathrm{psi}$ \\
\hline Length & 6 in & Part Type & 3-D Solid \\
\hline Width & $1.5-3$ in & Section Type & 3-D Solid \\
\hline Height & 0.375 in & -- & --- \\
\hline
\end{tabular}

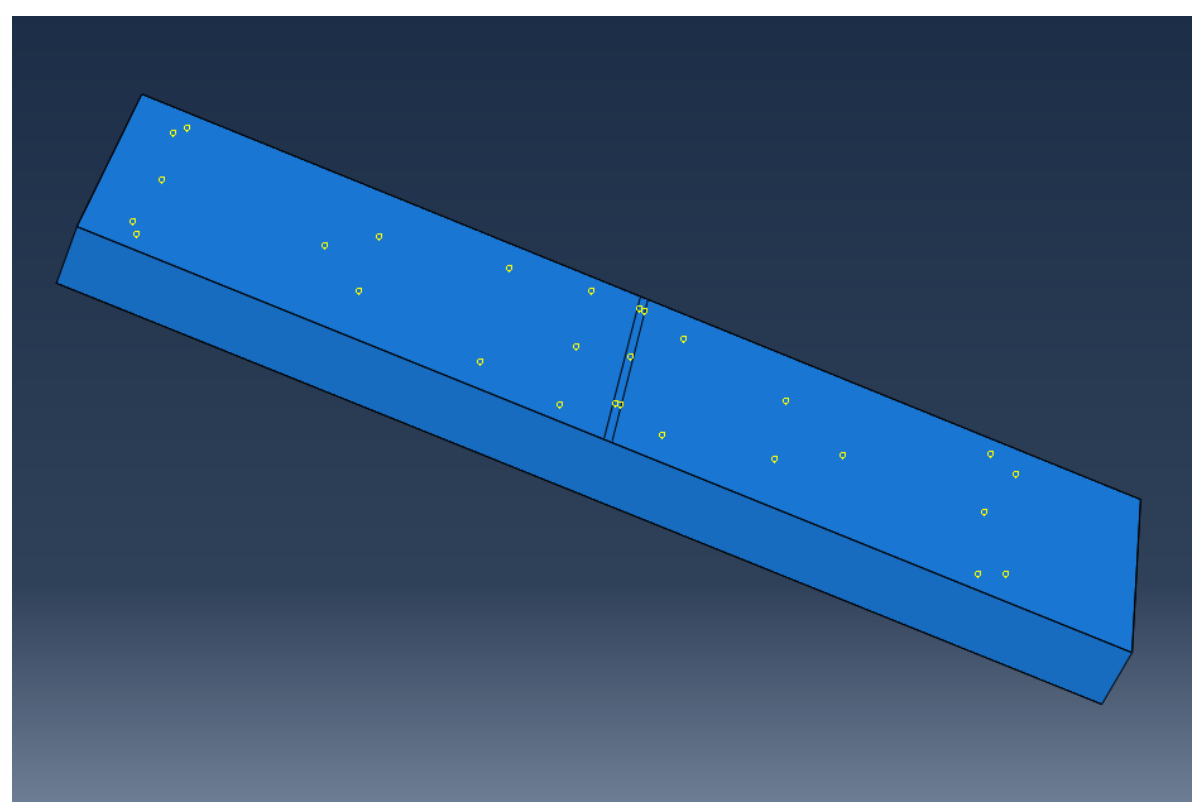

Figure 5.1. FEA Tie constraints on top and bottom shell elements. 
The standard units were also English, but the pressures were measured in pounds per square inch (psi). The composite beam was modeled as two separate parts, the core as a 3-D deformable solid and the face sheets as a 3-D shell. A shell section was created with the carbon fiber face sheets with the required shell thickness defined as the face sheet thickness and a solid section was created with the honeycomb core from the mechanical properties shown in Table 5.2 above. The face sheets were constructed as two individual parts and in order to simulate the layers through the specified thickness of the face sheet, 9 integration points were stacked vertically from the mid-plane of the element and define the thickness of each layer within the laminate. Laminated shell theory was applied for the assumption that a uniform constant shear strain through the thickness of the shell occurs. If this correction is not applied, the stiffness of the shell can be highly inaccurate if drastic differences in elastic constants between ply are present, especially in sandwich type shells.

In the assembly the instances were translated to their respective coordinates before face and edge constraints were applied. As the bonding strength of the carbon fiber resin between the carbon

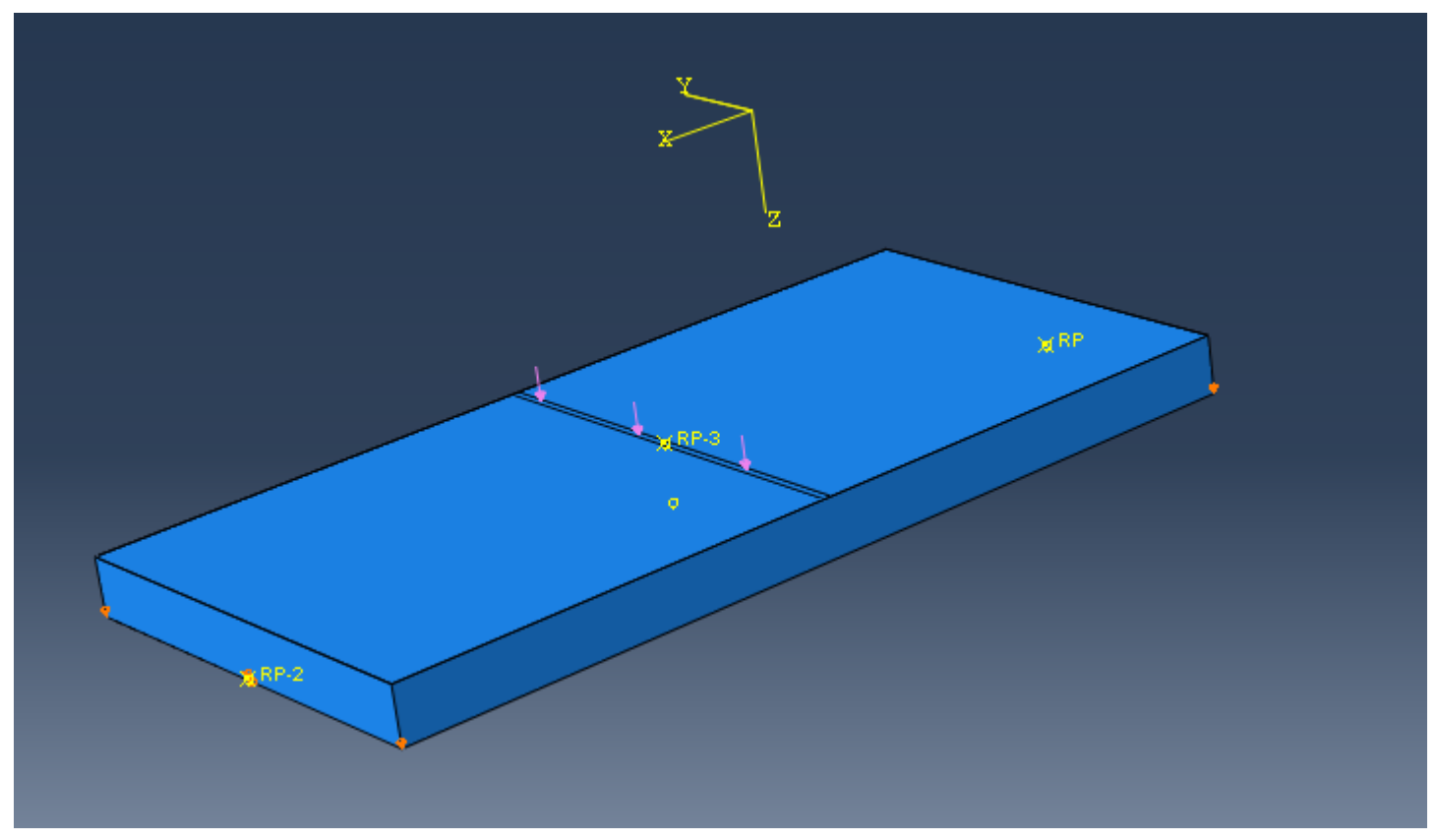

Figure 5.2. FEA Boundary Conditions and Loads. 
fiber and the core is unknown, tie constraints shown Figure 5.1 were created in order to allow for shared nodes between face sheets and core. The first tie had the top surface of the core as the master and the bottom surface of the top face sheet as the slave. The second tie had the bottom surface of the core as the slave and the top surface of the bottom face sheet as the master. Boundary conditions were applied to both bottom edges parallel to the applied load. Both sides constrained displacement in U3 or the $z$ orientation and displacement in the $\mathrm{U} 2$ or $\mathrm{y}$ direction was constrained at the center of the edges only, to allow deflections along the perpendicular edges to the applied load due to the anticlastic behavior of the core seen figure 5.2 .

Once an FEA model was confirmed using materials with a known theoretical deflection was confirmed, the model could then be adapted for the experimental materials. Four node plate elements with orthotropic material properties (See Tables 5.1-2) were used to model the face and core laminates. It must be noted that this is a simplified model of the real structure, particularly with the vary face sheet thickness as the extension-bending coupling is ignored. This simplification can be justified because the face laminates are located far enough from the neutral axis relative to its thickness, that the deformation of the cross-section from flexure results in a near uniform strain distribution over the thickness of the faces. Thus we can consider the faces as being close to only extensional loading.

A study into element types was conducted in order to obtain deflection and stress results as close to theoretical values as possible seen table 5.3 . 
Table 5.3. Element Study

\begin{tabular}{|c|c|c|c|c|c|c|c|c|}
\hline & \multicolumn{2}{|c|}{ Theoretical } & \multicolumn{2}{|c|}{ Option 1} & \multicolumn{2}{|c|}{ Option 2} & \multicolumn{2}{|c|}{ Option 3} \\
\hline & Core & Face & Core & Face & Core & Face & Core & Face \\
\hline Element Type & - & - & $\begin{array}{c}\text { Quad: } \\
\text { Struct } \\
\text { Hex }\end{array}$ & $\begin{array}{c}\text { S4 } \\
\text { Quad: } \\
\text { Free }\end{array}$ & $\begin{array}{c}\text { Quad: } \\
\text { Struct } \\
\text { Hex }\end{array}$ & $\begin{array}{c}\text { S4 } \\
\text { Quad: } \\
\text { Free }\end{array}$ & $\begin{array}{c}\text { Quad: } \\
\text { Struct } \\
\text { Hex }\end{array}$ & $\begin{array}{c}\text { S4 } \\
\text { Quad: } \\
\text { Free }\end{array}$ \\
\hline $\begin{array}{c}\text { Standard or } \\
\text { Explicit }\end{array}$ & - & - & $S$ & $S$ & $S$ & $S$ & $E$ & $S$ \\
\hline $\begin{array}{c}\text { Reduced } \\
\text { Integration on }\end{array}$ & - & - & & & & & & $x$ \\
\hline $\begin{array}{l}\text { Linear or } \\
\text { Quadratic }\end{array}$ & - & - & $\mathrm{L}$ & L & L & $\mathrm{L}$ & Q & $\mathrm{L}$ \\
\hline $\begin{array}{c}\text { Hourglass } \\
\text { Control }\end{array}$ & - & - & $x$ & & $x$ & & & \\
\hline $\begin{array}{l}\text { Incompressible } \\
\text { Modes on }\end{array}$ & - & - & & & $x$ & & & \\
\hline $\begin{array}{c}\text { Midspan } \\
\text { Deflection (in) }\end{array}$ & \multicolumn{2}{|c|}{0.017499} & \multicolumn{2}{|c|}{0.0168028} & \multicolumn{2}{|c|}{0.0167711} & \multicolumn{2}{|c|}{0.0184261} \\
\hline \multirow[t]{3}{*}{ Error (\%) } & \multicolumn{2}{|c|}{-} & \multicolumn{2}{|c|}{$3.979 \%$} & \multicolumn{2}{|c|}{$4.16 \%$} & \multicolumn{2}{|c|}{$5.29 \%$} \\
\hline & \multicolumn{2}{|c|}{ Option 4} & \multicolumn{2}{|c|}{ Option 5} & \multicolumn{2}{|c|}{ Option 6} & \multicolumn{2}{|c|}{ Option 7} \\
\hline & Core & Face & Core & Face & Core & Face & Core & Face \\
\hline Element Type & $\begin{array}{c}\text { Quad: } \\
\text { Struct } \\
\text { Hex }\end{array}$ & $\begin{array}{c}\text { S4 } \\
\text { Quad: } \\
\text { Free }\end{array}$ & $\begin{array}{l}\text { Tetrhl: } \\
\text { Struct }\end{array}$ & $\begin{array}{c}\text { S4 } \\
\text { Quad: } \\
\text { Free }\end{array}$ & $\begin{array}{c}\text { Quad: } \\
\text { Struct } \\
\text { Hex }\end{array}$ & $\begin{array}{c}\text { S4 } \\
\text { Quad: } \\
\text { Struct }\end{array}$ & $\begin{array}{c}\text { Quad: } \\
\text { Struct } \\
\text { Hex }\end{array}$ & $\begin{array}{c}\text { S4 } \\
\text { Quad: } \\
\text { Free } \\
\end{array}$ \\
\hline $\begin{array}{l}\text { Standard or } \\
\text { Explicit }\end{array}$ & $S$ & $S$ & $E$ & $S$ & $S$ & $S$ & $S$ & $S$ \\
\hline $\begin{array}{c}\text { Reduced } \\
\text { Integration on }\end{array}$ & $x$ & $x$ & $x$ & $x$ & $x$ & $x$ & $x$ & $x$ \\
\hline Linear or quad & $Q$ & L & $Q$ & L & L & $Q$ & L & L \\
\hline $\begin{array}{c}\text { Hourglass } \\
\text { Control }\end{array}$ & $x$ & & $x$ & & $x$ & & $x$ & \\
\hline \multicolumn{9}{|l|}{$\begin{array}{l}\text { Incompressible } \\
\text { Modes on }\end{array}$} \\
\hline $\begin{array}{c}\text { Midspan } \\
\text { Deflection (in) }\end{array}$ & \multicolumn{2}{|c|}{0.0192311} & \multicolumn{2}{|c|}{0.02663} & \multicolumn{2}{|c|}{0.016166} & \multicolumn{2}{|c|}{0.016869} \\
\hline Error & \multicolumn{2}{|c|}{$9.89 \%$} & \multicolumn{2}{|c|}{$52.18 \%$} & \multicolumn{2}{|c|}{$7.6176 \%$} & \multicolumn{2}{|c|}{$3.6 \%$} \\
\hline
\end{tabular}




\begin{tabular}{|c|c|c|c|c|}
\hline & \multicolumn{2}{|c|}{ Option 8} & \multicolumn{2}{|c|}{ Option 9} \\
\hline & Core & Face & Core & Face \\
\hline Element Type & $\begin{array}{c}\text { Quad: } \\
\text { Struct } \\
\text { Hex }\end{array}$ & $\begin{array}{c}\text { S4 } \\
\text { Quad: } \\
\text { Free }\end{array}$ & $\begin{array}{c}\text { Quad: } \\
\text { Struct } \\
\text { Hex }\end{array}$ & $\begin{array}{c}\text { S4 } \\
\text { Quad: } \\
\text { Free }\end{array}$ \\
\hline $\begin{array}{l}\text { Standard or } \\
\text { Explicit }\end{array}$ & $S$ & $S$ & $S$ & $S$ \\
\hline $\begin{array}{c}\text { Reduced } \\
\text { Integration on }\end{array}$ & $x$ & & $x$ & \\
\hline Linear or quad & L & L & $\mathrm{L}$ & L \\
\hline $\begin{array}{l}\text { Hourglass } \\
\text { Control }\end{array}$ & $x$ & & $x$ & \\
\hline $\begin{array}{c}\text { Incompressible } \\
\text { Modes on }\end{array}$ & & & $x$ & \\
\hline $\begin{array}{c}\text { Midspan } \\
\text { Deflection (in) }\end{array}$ & \multicolumn{2}{|c|}{0.0168906} & \multicolumn{2}{|c|}{0.016769} \\
\hline Error (\%) & \multicolumn{2}{|c|}{$3.477 \%$} & \multicolumn{2}{|c|}{$4.172 \%$} \\
\hline
\end{tabular}

The chosen element types was option 8 with the core being a C3D8R 8 node, standard, linear model, structured quadratic element type with reduced integration and hourglass control. The face element was S4: 4 node doubly curved generally purpose shell, free structured element type with finite member strains and produced an error of $3.477 \%$ with the theoretical model.

\subsection{Static Loading Analysis}

The finite element models of the composite sandwich were modeled identically to the experimental models with the same static loading boundary conditions. Using this method, the FE maximum/mid-span deflection results could be compared with theoretical hand calculations by analysis 
a closed form system. The model was layered with two steel face sheets between an aluminum core with mechanical properties shown in Table 5.4 .

Table 5.4. Mechanical and part properties used for FEA model validation

\begin{tabular}{|l|l|l|l|l|l|l|l|l|}
\hline Part & Material & $\begin{array}{l}\text { E } \\
\text { (Msi) }\end{array}$ & $\begin{array}{l}\text { Poisson's } \\
\text { Ratio }\end{array}$ & $\begin{array}{l}\text { Length } \\
\text { (in) }\end{array}$ & $\begin{array}{l}\text { Width } \\
\text { (in) }\end{array}$ & $\begin{array}{l}\text { Depth } \\
\text { (in) }\end{array}$ & $\begin{array}{l}\text { Part } \\
\text { Type }\end{array}$ & $\begin{array}{l}\text { Section } \\
\text { Type }\end{array}$ \\
\hline Core & $\begin{array}{l}\text { Aluminum } \\
2024\end{array}$ & 10 & 0.3 & 6 & 1.5 & 0.375 & $\begin{array}{l}\text { 3-D } \\
\text { Solid }\end{array}$ & $\begin{array}{l}\text { 3-D } \\
\text { Solid }\end{array}$ \\
\hline $\begin{array}{l}\text { Face } \\
\text { Sheets }\end{array}$ & $\begin{array}{l}\text { Steel } \\
1018\end{array}$ & 29 & 0.297 & 6 & 1.5 & 0.024 & $\begin{array}{l}\text { 3-D } \\
\text { Shell }\end{array}$ & $\begin{array}{l}\text { 3-D } \\
\text { Shell }\end{array}$ \\
\hline
\end{tabular}

The structure was then loaded with a 100lb mid-span force. The total load was chosen to be $100 \mathrm{lb}$ to lie in the linear elastic region of the load - deflection curve for all configurations as determined by experimental results, this load also is kept in the range of the simulation assumption of small angle deflections.

\subsection{Validation of FEA Model}

The static load case above was used to compare FEA results with theoretical hand calculations. The method of equivalent stiffness's was used for theoretical deflections and MATLAB code can be seen in the apendix..$^{33}$ The elastic modulus of steel is three times as much as aluminum, an equivalent cross section of aluminum can be made by replacing the steel with aluminum that is three times as large. The equation for the mid-span or maximum deflection for a simply supported beam under three point bending can be seen Equation 3.3 in section 3.2. The FEA results came reasonably close to theoretical results seen in Table 5.5. 
Table 5.5. Comparison of Theoretical and Experimental deflection for model validation with Aluminium face sheets and Steel core.

\begin{tabular}{|l|l|}
\hline Model & Results \\
\hline Theoretical Deflection at 100 lbf (in) & 0.02 \\
\hline $\begin{array}{l}\text { FEA Maximum Deflection at 100lbf (in) } \\
\text { correlating to a seed size of 0.1 }\end{array}$ & 0.019997 \\
\hline Error (\%) & $0.01499 \%$ \\
\hline
\end{tabular}

From the acceptable results produced by the validation model, we can assume the model is sufficiently modelled in regards to boundary conditions, area of applied load and interactions between layers. Square linear elements were used to fit the rectangular geometry of the specimens.

\subsection{Mesh Convergence}

To check mesh convergence, to simulate a simply supported beam like that conducted experimentally. The $100 \mathrm{lb}$. load was modeled, as a pressure load with a total pressure of $100 \mathrm{psi}$. This area was chosen to emulate the three point bending loading fixture of $0.075 \mathrm{in}^{2}$. Two convergence

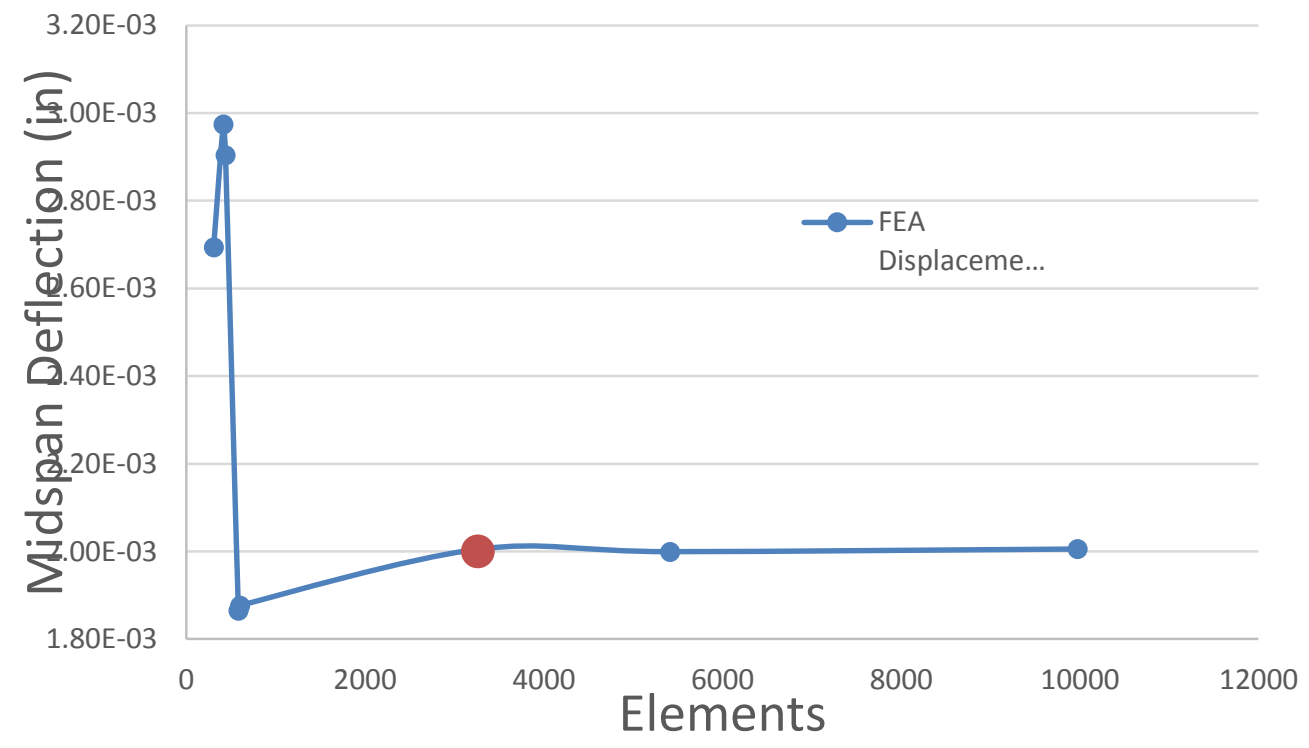

Figure 5.3. Convergence Plot for Midspan Beam deflection modelled in Abaqus 
studies were analyzed for mesh convergence, maximum deflection and stress of upper face of the top face sheet. Figure 5.3 shows the convergence plot for maximum deflection taken at the mid-span top surface of the top face sheet. The degrees of freedom was changed by altering the seed size between 0.06 and $0.3 \mathrm{in}$. The graph converges at 3263 elements indicated by the orange dot, corresponding to a seed size of 0.1. Further FEA analysis was then conducted with a part mesh of 0.1 in in the model. Linear elements were used in the 3-D solid element parts with reduced integration turned off so each element was analyzed as an 8 node element, which resulted in 32638 node elements in the entire model, with a seed size of 0.1 in. 


\subsection{Comparison of Experimental, Theoretical, and FEA Results}

This section covers the comparison of Experimental, Theoretical and Finite Element analysis.

\subsection{Composite Sandwich Panels with Varying Core Ribbon Orientation}

The three-point loading was applied to all configurations of the model shown in Fig.5.1. The stress and displacements at 100lbf for all configurations under three point bending can be seen in Table 6.1 below.

Table 6.1. Comparison of FEA, Experimental and Theoretical data for Core Ribbon Orientation variations

\begin{tabular}{|l|l|l|l|l|l|l|l|}
\hline $\begin{array}{c}\text { Core } \\
\text { Ribbon } \\
\text { Direction } \\
\text { (degrees) }\end{array}$ & $\begin{array}{c}\text { Maximum } \\
\text { Deflection } \\
\text { FEA (in) }\end{array}$ & $\begin{array}{c}\text { Maximum } \\
\text { Deflection } \\
\text { Theoretical } \\
\text { (in) }\end{array}$ & $\begin{array}{c}\text { Maximum } \\
\text { Deflection } \\
\text { Experimental } \\
\text { (in) }\end{array}$ & $\begin{array}{c}\text { Difference } \\
\text { Theoretical } \\
- \text { FEA } \\
(\%)\end{array}$ & $\begin{array}{c}\text { Difference } \\
\text { Theoretical - } \\
\text { Experimental } \\
\text { (\%) }\end{array}$ & $\begin{array}{c}\text { Difference } \\
\text { FEA- } \\
\text { Experimental } \\
\text { (\%) }\end{array}$ & $\begin{array}{l}\text { Maximum } \\
\text { Stress (ksi) }\end{array}$ \\
\hline $0^{\circ}$ & 0.03275 & 0.03517 & $\begin{array}{l}0.0337 \\
\pm 0.00078\end{array}$ & 6.88 & 4.18 & 2.99 & 10980 \\
\hline $15^{\circ}$ & 0.03431 & 0.03592 & $\begin{array}{l}0.0363 \\
\pm 0.0011\end{array}$ & 4.48 & 1.06 & 5.79 & 11140 \\
\hline $30^{\circ}$ & 0.03875 & 0.03826 & $\begin{array}{l}0.038 \\
\pm 0.0012\end{array}$ & 1.28 & 0.68 & 1.93 & 11100 \\
\hline $4^{\circ}$ & 0.04505 & 0.0423 & $\begin{array}{l}0.0453 \\
\pm 0.0013\end{array}$ & 6.5 & 7.09 & 0.55 & 10970 \\
\hline $60^{\circ}$ & 0.05157 & 0.04781 & $\begin{array}{l}0.0486 \\
\pm 9.13 e-4\end{array}$ & 7.86 & 1.65 & 5.76 & 12650 \\
\hline $75^{\circ}$ & 0.05646 & 0.05329 & $\begin{array}{l}0.0555 \\
\pm 0.0022\end{array}$ & 5.95 & 3.77 & 1.71 & 14060 \\
\hline $9^{\circ}$ & 0.05827 & 0.05575 & $\begin{array}{l}0.0558 \\
\pm 0.0019\end{array}$ & 4.52 & 0.09 & 4.25 & 14590 \\
\hline
\end{tabular}

The finite element results were observed to have minimal error when compared to the theoretical data, which confirmed that the model was acceptable. The maximum stress in the specimen increases with an increase in core orientation. This is due to two factors, the core's Young's moduli in the bending orientation decreases, taking less shear stress and there is a greater anticlastic curvature in the core at $90^{\circ}$ than $0^{\circ}$. This means that as Load is applied the rate at which the perpendicular edges to applied load inflect away from the applied load is greater at $90^{\circ}$ and so as the anticlastic curvature increases, the area of which load is applying decreases at a greater rate, causing stress concentrations at the perpendicular 
edges seen figure 2 . The $45^{\circ}$ core orientation has the anticlastic curvature at a $45^{\circ}$ degree angle to the applied load and thus applied load area reduction rate is at its minimum.

Finite element deflection results varied by no more than $5.76 \%$ from experimental results and as expected the maximum normal stress is located at the supports of the beam. A typical Beam deflection can be seen in Figure 6.2 below. Figure 6.1 shows the visual comparison between Experimental, analytical and numerical deflections at $100 \mathrm{lbf}$. It can be seen that beam and plate theory deflections are

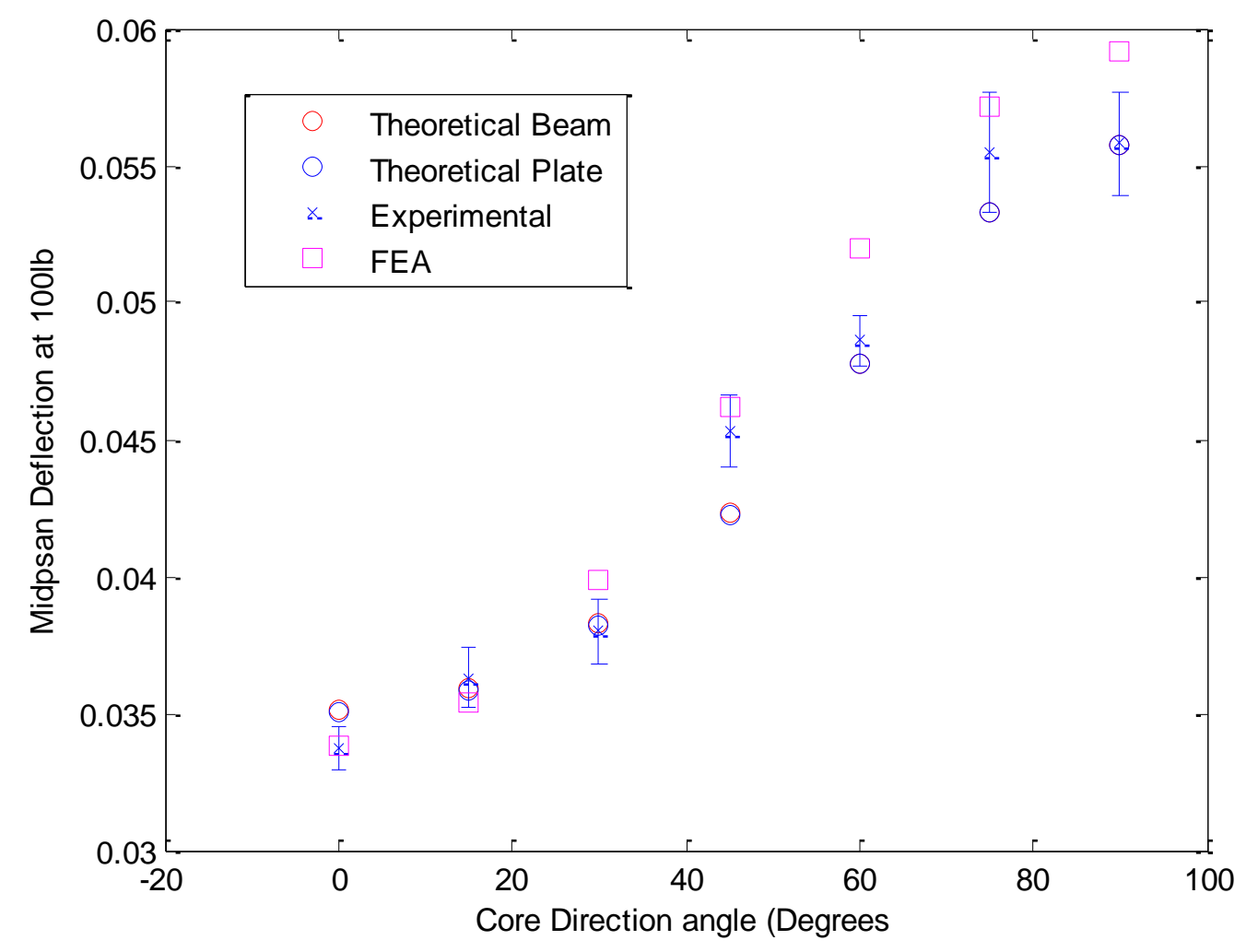

Figure 6.1. Comparison of Experimental, FEA, and Theoretical deflection for Varying Core Angle 


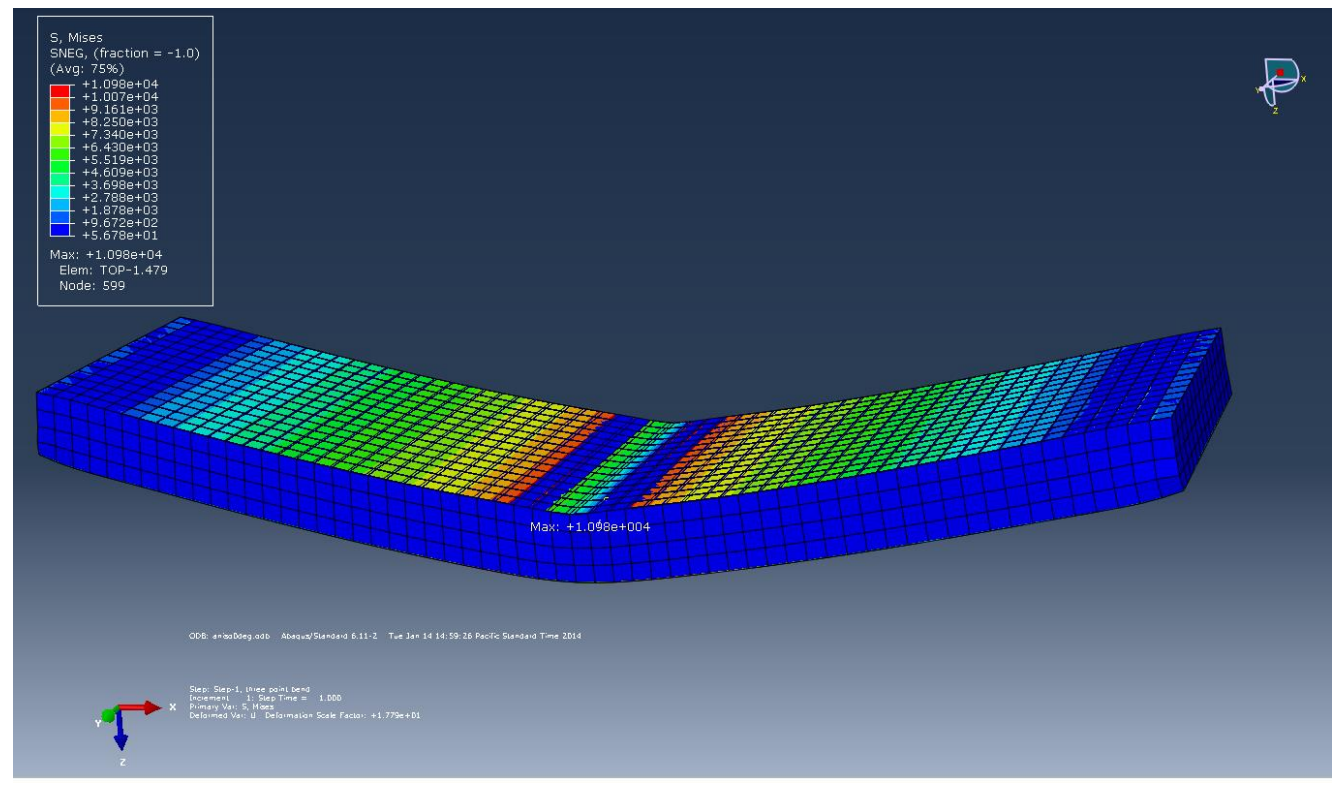

Figure 6.2. Stress distribution for Beam for Core orientation of 0 degrees at 100lbf applied load

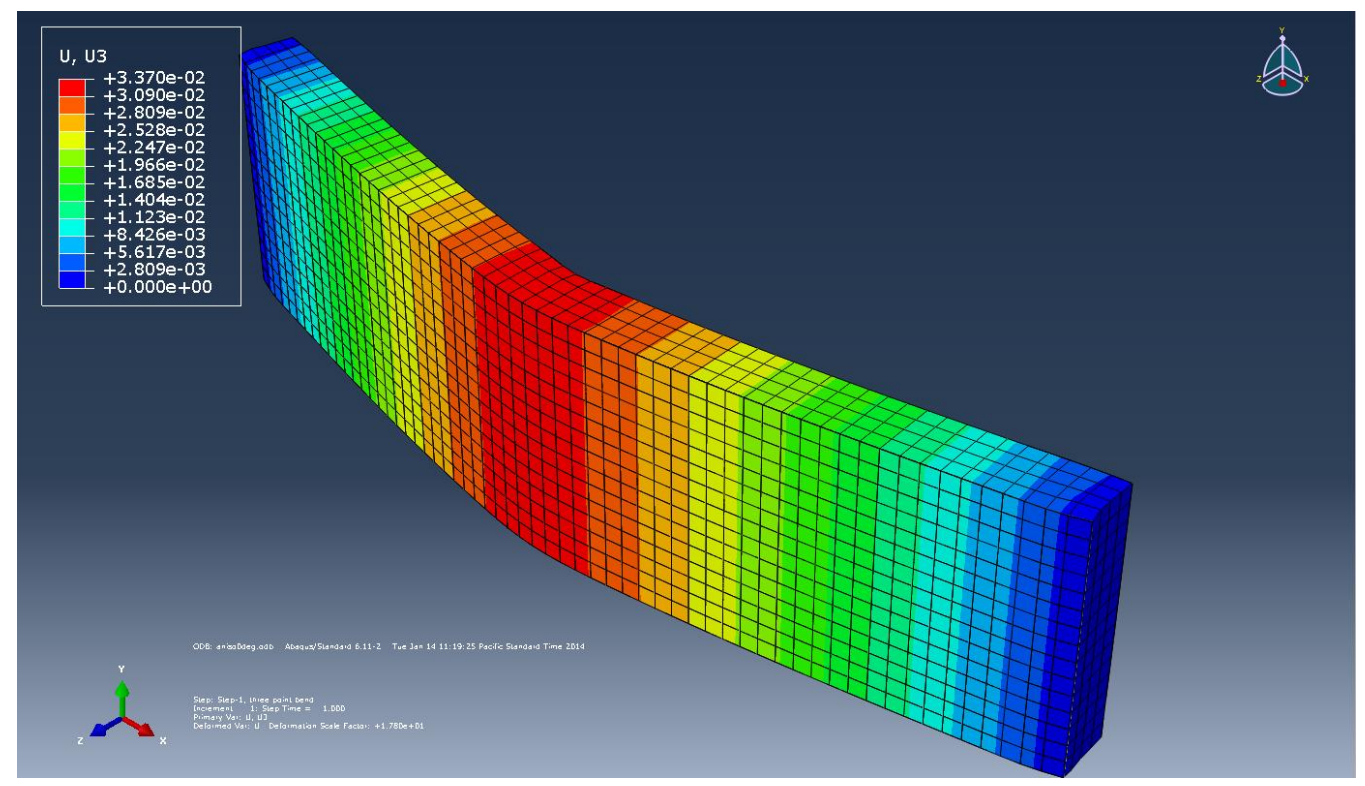

Figure 6.3. Deflection for Beam with Core orientation of 0 degrees at 100lbf applied load

almost identical. Finite element analysis had reasonable agreement with analytical results with an average percentage error of $5.837 \%$ and maximum percent error of $9.095 \%$ with Classical beam theory. The finite element model did marginally better with plate theory with an average percent error of 
$5.783 \%$ and a maximum error of $9.1761 \%$. Both maximum errors occurred at the $45^{\circ}$ core ribbon orientation. This is due to the mechanical properties of the honeycomb being estimated by using the transformation matrix to rotate the mechanical properties of the core to model the mechanical properties of the Nomex honeycomb core at $45^{\circ}$.

\subsection{Composite Sandwich Panels with Varying Face Sheet Thickness's}

The stress and displacements for all configurations under three point bending can be seen in Table 6.2 below.

Table 6.2. Comparison of FEA, Theoretical and Experimental Mid Span Deflections at $100 \mathrm{lbf}$

\begin{tabular}{|c|c|c|c|c|c|c|c|}
\hline & \multicolumn{7}{|c|}{ Top Face Sheet Thickness (in) } \\
\hline \multirow{16}{*}{$\begin{array}{l}\text { Bottom } \\
\text { Face } \\
\text { Sheet } \\
\text { Thicknes } \\
\text { s (in) }\end{array}$} & & & $\begin{array}{c}0.012 \\
\text { (1 Layer) }\end{array}$ & $\begin{array}{c}0.024 \\
\text { (2 Layers) }\end{array}$ & $\begin{array}{c}0.036 \\
\text { (3 Layers) }\end{array}$ & $\begin{array}{c}0.048 \\
\text { (4 Layers) }\end{array}$ & $\begin{array}{c}0.06 \\
\text { (5 Layers) }\end{array}$ \\
\hline & \multirow[t]{3}{*}{$\begin{array}{c}0.012 \\
\text { (1 Layer) }\end{array}$} & Experimental & $\begin{array}{l}0.0265 \\
\pm 0.002\end{array}$ & $\begin{array}{c}0.0201 \\
\pm 0.0003\end{array}$ & $\begin{array}{c}0.0185 \\
\pm 0.0003\end{array}$ & $\begin{array}{c}0.0164 \\
\pm 0.0002\end{array}$ & $\begin{array}{c}0.0149 \\
\pm 0.0002\end{array}$ \\
\hline & & Theoretical & 0.0247 & 0.0201 & 0.0177 & 0.0161 & 0.0149 \\
\hline & & FEA & 0.0242 & 0.0205 & 0.0192 & 0.0182 & 0.0176 \\
\hline & \multirow{3}{*}{$\begin{array}{c}0.024 \\
(2 \\
\text { Layers) }\end{array}$} & Experimental & $\begin{array}{c}0.0231 \\
\pm 0.0006\end{array}$ & $\begin{array}{c}0.0169 \\
\pm 0.0005\end{array}$ & $\begin{array}{c}0.0155 \\
\pm 0.0004\end{array}$ & $\begin{array}{c}0.0139 \\
\pm 0.0005\end{array}$ & $\begin{array}{c}0.0127 \\
\pm 0.0011\end{array}$ \\
\hline & & Theoretical & 0.0201 & 0.0175 & 0.0159 & 0.0147 & 0.0138 \\
\hline & & FEA & 0.0204 & 0.0168 & 0.0155 & 0.0147 & 0.0141 \\
\hline & \multirow{3}{*}{$\begin{array}{c}0.036 \\
\text { (3 } \\
\text { Layers) }\end{array}$} & Experimental & $\begin{array}{c}0.0193 \\
\pm 0.0008\end{array}$ & $\begin{array}{c}0.0152 \\
\pm 0.0004\end{array}$ & $\begin{array}{c}0.0141 \\
\pm 0.0002\end{array}$ & $\begin{array}{c}0.0137 \\
\pm 0.0005\end{array}$ & $\begin{array}{c}0.0131 \\
\pm 0.0003\end{array}$ \\
\hline & & Theoretical & 0.0177 & 0.0159 & 0.0146 & 00137 & 0.0130 \\
\hline & & FEA & 0.0189 & 0.0155 & 0.0142 & 00134 & 0.0128 \\
\hline & \multirow{3}{*}{$\begin{array}{c}0.048 \\
(4 \\
\text { Layers) }\end{array}$} & Experimental & $\begin{array}{c}0.0176 \\
\pm 0.0003\end{array}$ & $\begin{array}{c}0.0147 \\
\pm 0.0004\end{array}$ & $\begin{array}{c}0.0134 \\
\pm 0.0006\end{array}$ & $\begin{array}{c}0.0127 \\
\pm 0.0004\end{array}$ & $\begin{array}{c}0.0124 \\
\pm 0.0004\end{array}$ \\
\hline & & Theoretical & 0.0161 & 0.0147 & 0.0137 & 0.0129 & 0.0123 \\
\hline & & FEA & 0.0182 & 0.0147 & 0.0133 & 0.0126 & 0.0121 \\
\hline & \multirow{3}{*}{$\begin{array}{c}0.06 \\
(5 \\
\text { Layers) }\end{array}$} & Experimental & $\begin{array}{c}0.0178 \\
\pm 0.0007\end{array}$ & $\begin{array}{c}0.0132 \\
\pm 0.0007\end{array}$ & $\begin{array}{c}0.0126 \\
\pm 0.0003\end{array}$ & $\begin{array}{c}0.0116 \\
\pm 0.0006\end{array}$ & $\begin{array}{c}0.0117 \\
\pm 0.0007\end{array}$ \\
\hline & & Theoretical & 0.0149 & 0.0138 & 0.0130 & 0.0123 & 0.0117 \\
\hline & & FEA & 0.0175 & 0.0141 & 0.0129 & 0.0121 & 0.0116 \\
\hline
\end{tabular}


Finite element analysis showed reasonable agreement with theoretical and experimental midspan deflections. Specimens with a top to bottom face sheet ratio outside of $0.4 \leq t_{t} / t_{b} \leq 3$ produce greater error due to assumption that the bending-extension coupling matrix being zero is no longer valid due to the sandwich structure no longer being close to symmetric. Specimens outside the specified face sheet ratio range have been highlighted pink. Figure 6.4 shows a three dimensional curve fitting to finite element analysis performed on unequal face sheet configurations.

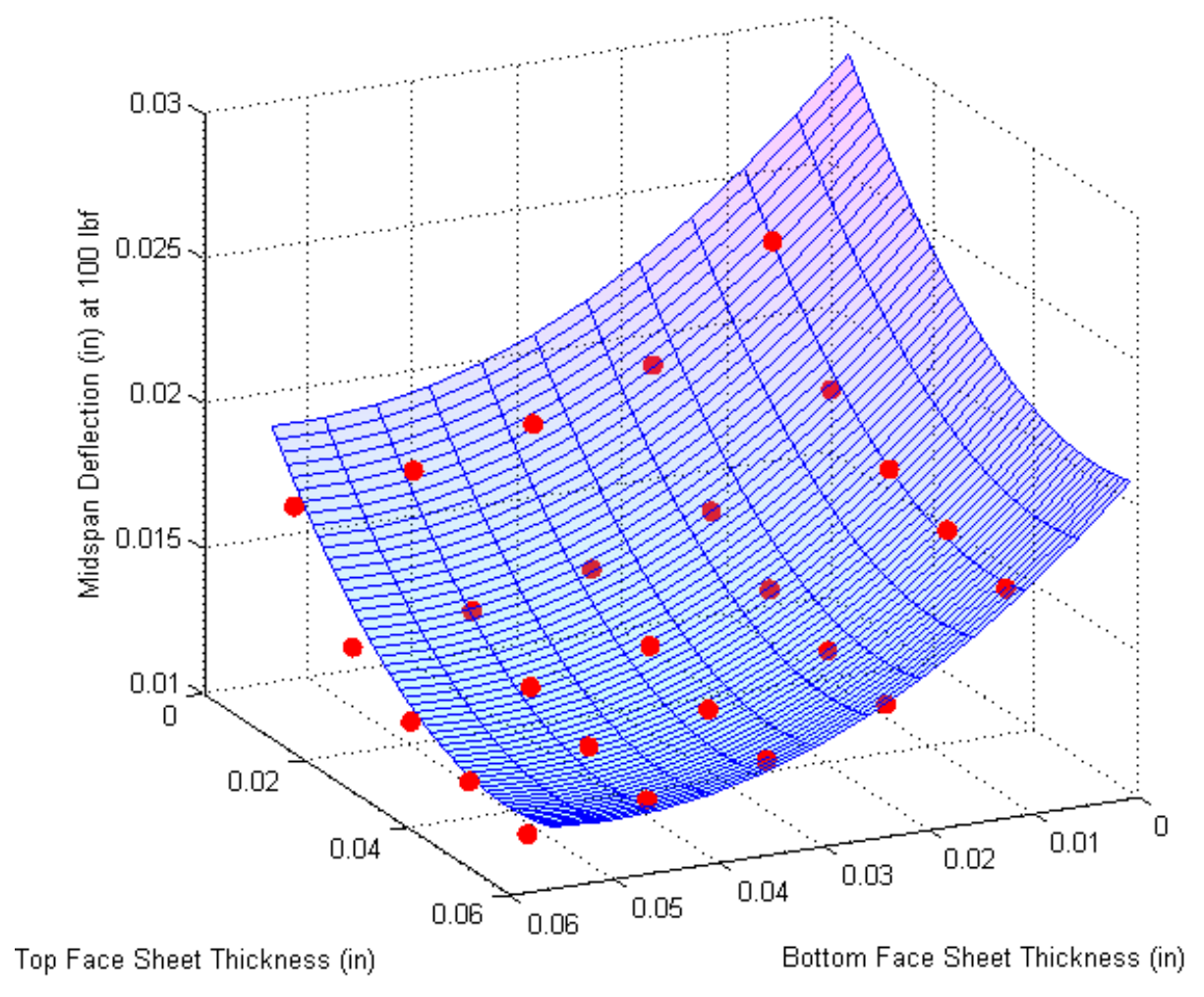

Figure 6.4. 3D Curve Fitting for FEA Deflections at $100 \mathrm{lbf}$. 
Table 6.3. Difference Between Finite Element and Theoretical Midspan Deflection for Varying Face Sheet Thickness at $100 \mathrm{lbf}$

\begin{tabular}{|c|c|c|c|c|c|c|}
\hline & \multicolumn{5}{|c|}{ Top Face Sheet Thickness (in) } \\
\hline \multirow{3}{*}{ Bottom } & & $\mathbf{0 . 0 1 2}$ & $\mathbf{0 . 0 2 4}$ & $\mathbf{0 . 0 3 6}$ & $\mathbf{0 . 0 4 8}$ & $\mathbf{0 . 0 6}$ \\
\cline { 2 - 7 } & $\mathbf{0 . 0 1 2}$ & $2.06 \%$ & $2.175 \%$ & $8.44 \%$ & $13.14 \%$ & $17.97 \%$ \\
\cline { 2 - 7 } Face Sheet & $\mathbf{0 . 0 2 4}$ & $1.28 \%$ & $3.87 \%$ & $2.38 \%$ & $0.12 \%$ & $1.94 \%$ \\
\cline { 2 - 7 } Thickness & $\mathbf{0 . 0 3 6}$ & $7.23 \%$ & $2.62 \%$ & $3.34 \%$ & $2.43 \%$ & $1.11 \%$ \\
\cline { 2 - 7 } (in) & $\mathbf{0 . 0 4 8}$ & $12.95 \%$ & $0.34 \%$ & $3.27 \%$ & $2.33 \%$ & $1.74 \%$ \\
& $\mathbf{0 . 0 6}$ & $17.11 \%$ & $2.29 \%$ & $0.9 \%$ & $2.45 \%$ & $1.25 \%$ \\
\cline { 2 - 7 } & & & & & & \\
\hline
\end{tabular}

Finite element deflections had better agreement with experimental deflections than theoretical

deflections indicating an agreeable model. As expected specimens above the face sheet thickness ratio of 3 produced larger errors. Interestingly the finite element model had agreement with experimental specimens with face sheet thickness ratio under 0.4

Table 6.4. Difference Between Finite Element and Experimental Midspan Deflection for Varying Face Sheet Thickness at $100 \mathrm{lbf}$

\begin{tabular}{|c|c|c|c|c|c|c|}
\hline & \multicolumn{7}{|c|}{ Top Face Sheet Thickness (in) } \\
\hline \multirow{3}{*}{ Bottom } & & $\mathbf{0 . 0 1 2}$ & $\mathbf{0 . 0 2 4}$ & $\mathbf{0 . 0 3 6}$ & $\mathbf{0 . 0 4 8}$ & $\mathbf{0 . 0 6}$ \\
\cline { 2 - 7 } & $\mathbf{0 . 0 1 2}$ & $9.79 \%$ & $1.95 \%$ & $3.40 \%$ & $10.00 \%$ & $15.63 \%$ \\
\cline { 2 - 7 } Face Sheet & $\mathbf{0 . 0 2 4}$ & $13.27 \%$ & $0.32 \%$ & $0.26 \%$ & $5.64 \%$ & $10.05 \%$ \\
\cline { 2 - 7 } Thickness & $\mathbf{0 . 0 3 6}$ & $1.87 \%$ & $1.35 \%$ & $0.39 \%$ & $2.50 \%$ & $2.21 \%$ \\
\cline { 2 - 7 } (in) & $\mathbf{0 . 0 4 8}$ & $3.27 \%$ & $0.16 \%$ & $0.64 \%$ & $0.58 \%$ & $2.71 \%$ \\
\cline { 2 - 7 } & $\mathbf{0 . 0 6}$ & $1.69 \%$ & $6.83 \%$ & $2.38 \%$ & $4.22 \%$ & $0.84 \%$ \\
\hline
\end{tabular}

Figures 6.5-9 visually show the differences between specimens keeping the bottom layer constant. 


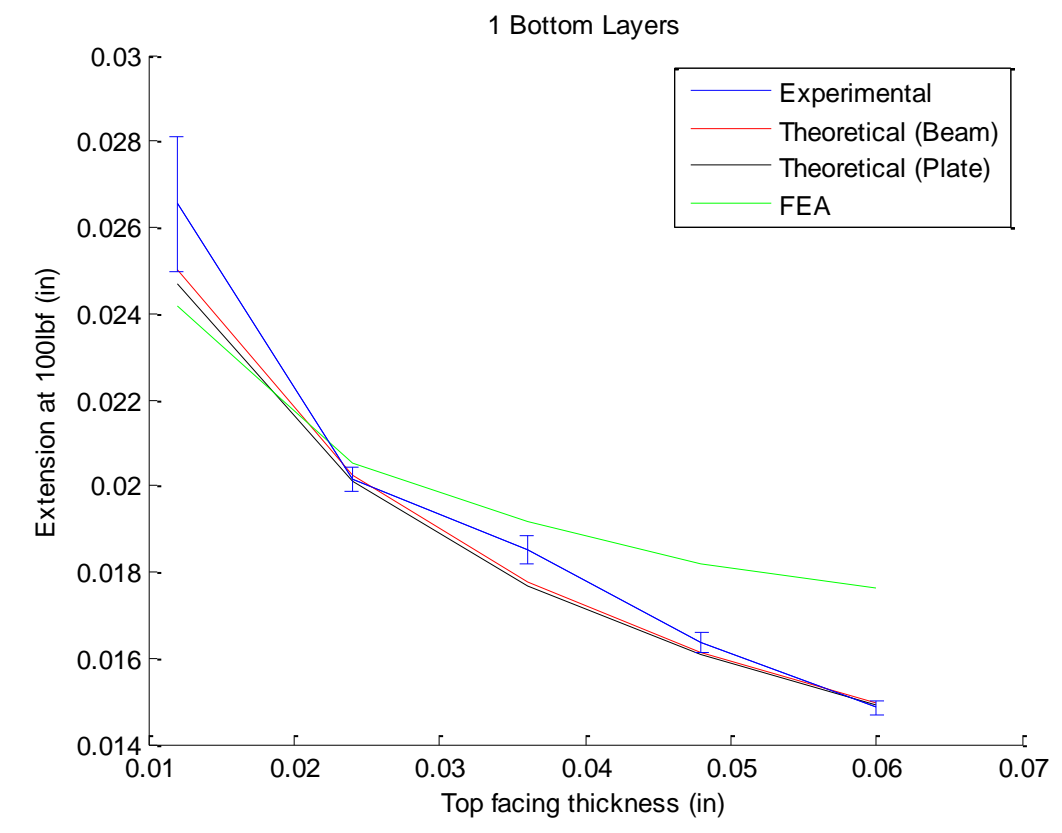

Figure 6.5. FEA, Experimental and Analytical Comparison for all configurations with 0.012in bottom layer at $100 \mathrm{lbf}$ load

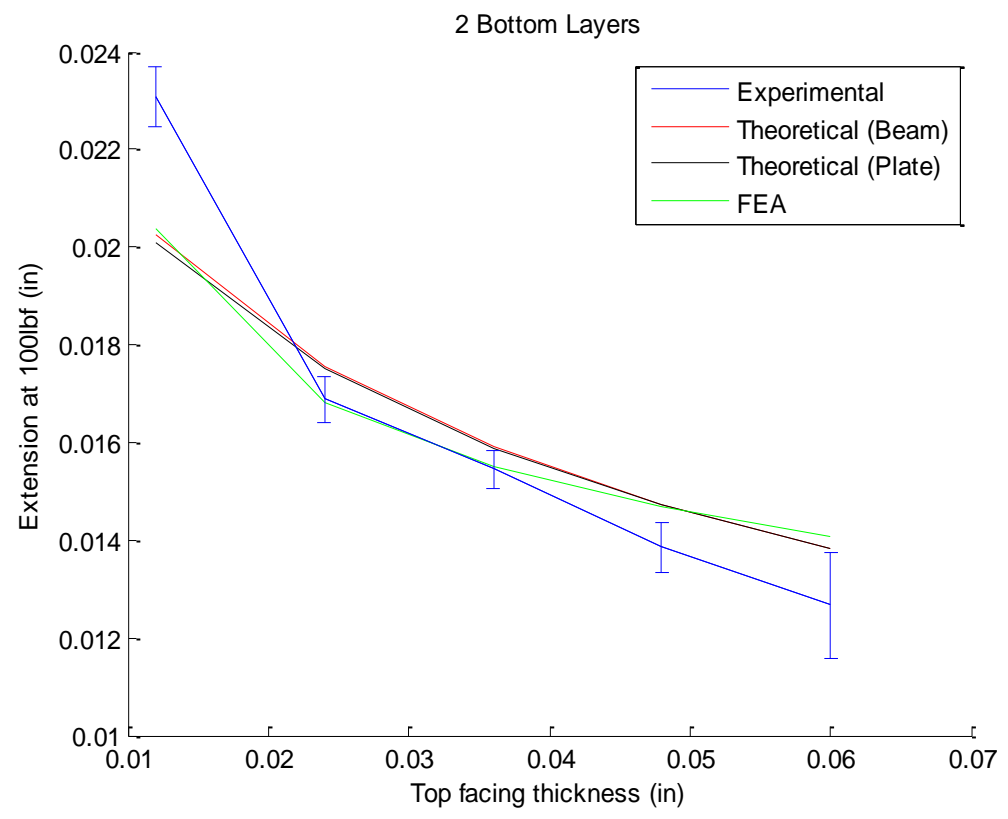

Figure 6.6. FEA, Experimental and Analytical Comparison for all configurations with 0.012in bottom layer at $100 \mathrm{lbf}$ load 


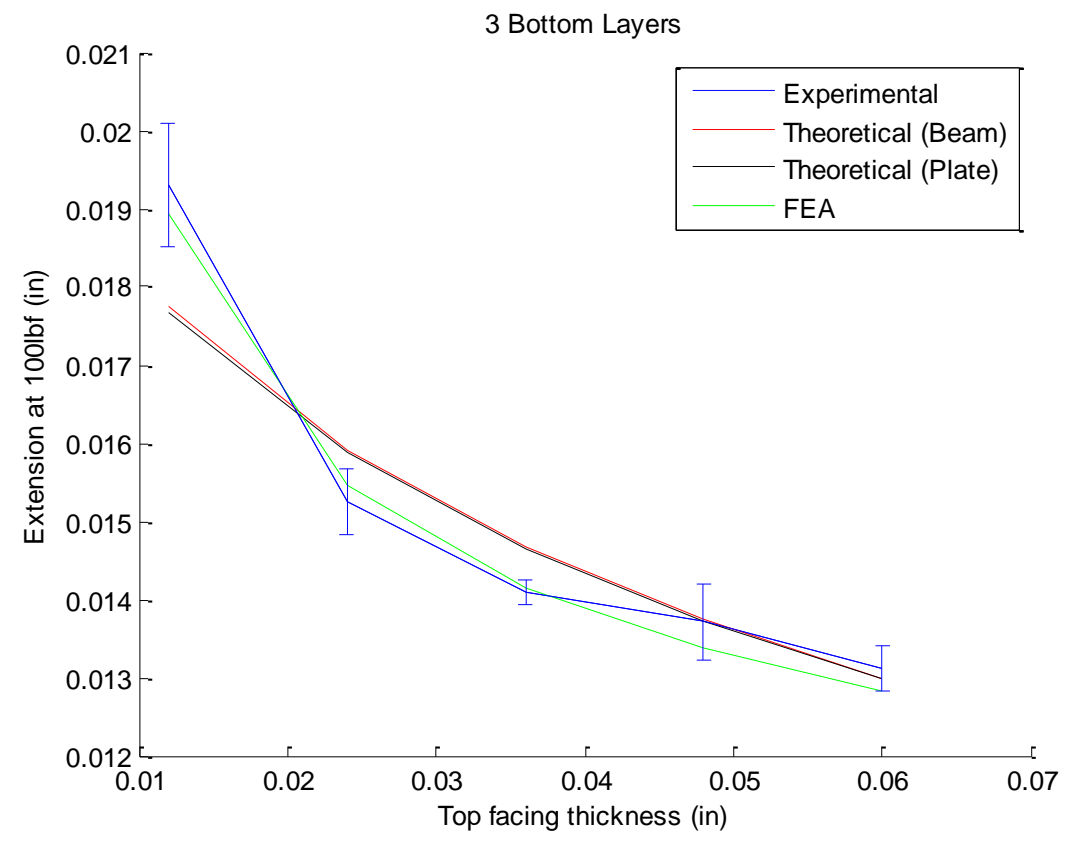

Figure 6.7. FEA, Experimental and Analytical Comparison for all configurations with 0.024in bottom layer at 100lbf load

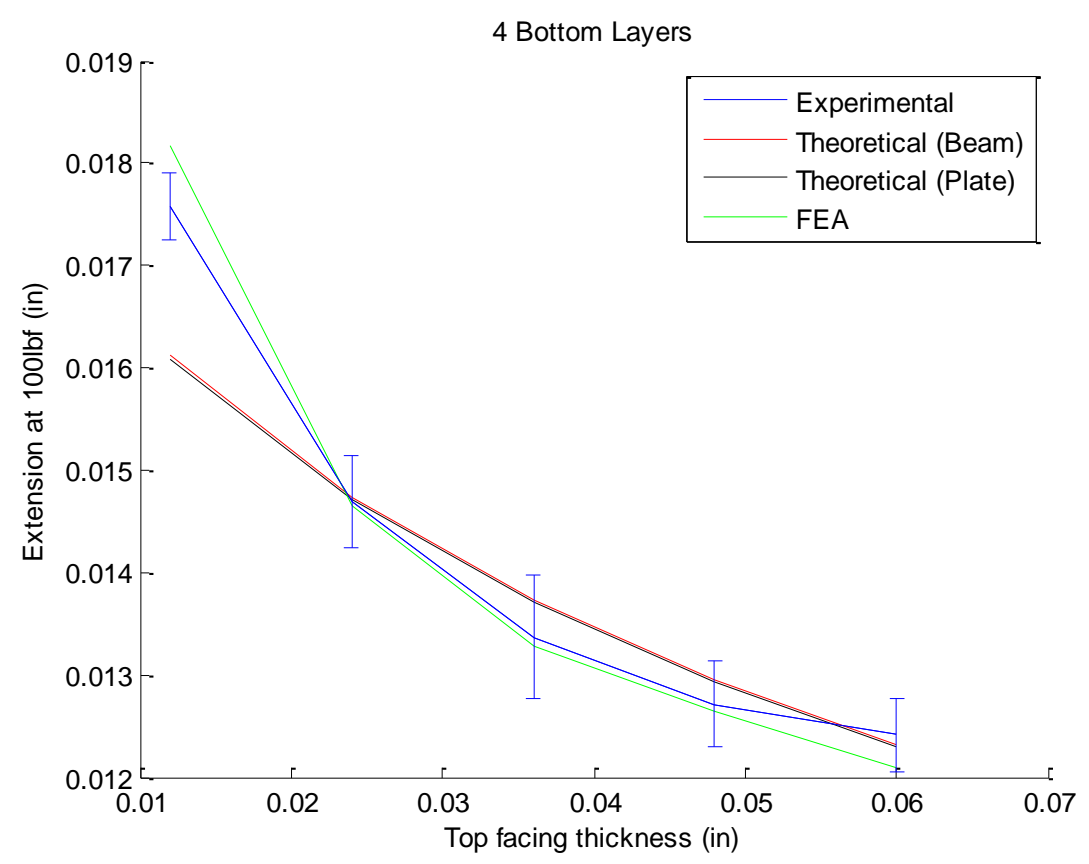

Figure 6.8. FEA, Experimental and Analytical Comparison for all configurations with 0.048in bottom layer 


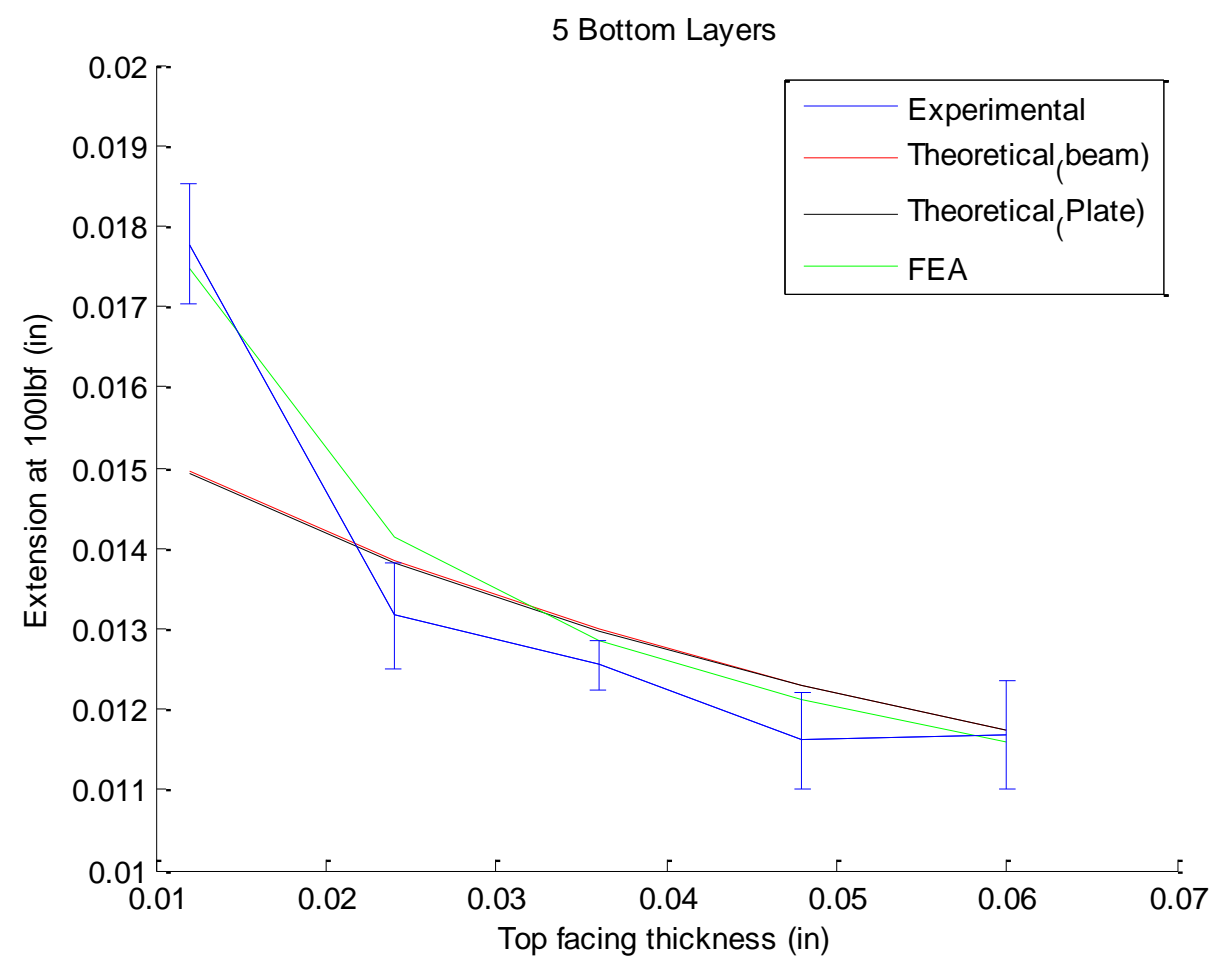

Figure 6.9. FEA, Experimental and Analytical Comparison for all configurations with $0.06 \mathrm{in}$ bottom layer at 100lbf load 


\subsection{Conclusion}

The objective of this study was to determine the effects on the mechanical behavior of sandwich structures with varying the core ribbon orientation of the honeycomb in composite honeycomb structures as well as varying face sheet thickness's with constant core subjected to three-point bend loadings. Nine core orientations from $0^{\circ}$ to $90^{\circ}$ degrees were tested, with test data available in the appendix. Twenty five specimen configurations with unique face sheet thickness varying from 0.012 to 0.06 in were also tested, with data available in the appendix. In addition to testing of sandwich structures ASTM standard tests were conducted to obtain the mechanical properties of the constituent materials. From the analytical and experimental work presented in this thesis the following conclusions were found:

- A simplified procedure for stiffness calculations, based on material properties found by testing under ASTM standards, was found to predict deflections within approximately $17 \%$ accuracy.

- The initiation of failure modes in composite sandwich structures is dependent on the constituent material properties, geometric dimensional and type of loading. Failure of Nomex honeycomb carbon fiber sandwich structures occurs by failure of the facing in compression of the core in shear and never due to face wrinkling.

- For relatively small beam spans and high face sheet to core thickness ratio, the predominant failure mode is core shear, and is then dominated by core compression as the face sheet to core thickness ratio is reduced. As the beam span is increased face failure in compression becomes the dominant mode of failure.

- Core indentation from cross head movement only takes affect when face to core thickness is less than 0.032 which is equivalent to one ply for materials used in this thesis. Failure near load point due to core indentation was not modelled well. An adequate model of the contact area 
and core failure criteria coupled with higher order beam theory is necessary to improve indentation analysis for honeycomb core sandwich structures.

- Improving the consistency of the analytical determination of failure loads is still in need of work, particularly with predicting failure loads in specimens with unequal face sheet thicknesses.

- Ensuring better quality control in the manufacturing and testing procedures will greatly improve ultimate load capabilities and reduce the scatter of data.

- Adding a layer of sheet adhesive between honeycomb core and pre-impregnated carbon fiber face sheets had little effect on the mechanical properties of the structure. Adding the adhesive layer also increased the structures weight, thus having a negative effect on structure optimization.

- The ultimate shear strength of the core decreased in a sinusoidal behavior when the core ribbon orientation is increased from $0^{\circ}$ (longitudinal orientation) to $90^{\circ}$ (transverse orientation).

- Increasing the core ribbon orientation from $0^{\circ}$ (longitudinal orientation) to $90^{\circ}$ (transverse orientation) increased ultimate deflection at failure and decreased ultimate failure load.

- Maximum stress on facing sheet increases with increasing core ribbon orientation angle. This is due to the anticlastic curvature induced in the transverse orientation of the core under bending which is a function of poissons. The rate of transverse curvature increases with core orientation, due to the transformation of the poissons. Thus a greater increase in anticlastic curvature causes an increase in area reduction at the applied load, which results in a greater stress (higher stress concentration on the smaller area) on the beam than that of the stress at 0 degree core orientation for equivalent applied loads. 
- Deflection due to shear rigidity must be included in theoretical deflections of sandwich structures when the core's Young's modulus is much less than the facing Young's modulus in the bending orientation.

- Increasing bottom face sheet thickness along constant top face sheet thickness lines increases flexural rigidity, but does not increase stiffness with respect to minimum weight.

- Optimal face sheet thickness for a given core thickness is a function of core and face sheet densities and for materials used in this thesis optimal bending stiffness occurs when the core weight accounts for $2 / 3$ of the total sandwich weight.

- Specimens with a top to bottom face sheet ratio outside of $0.4 \leq t_{t} / t_{b} \leq 3$ produce greater error due to assumption that the bending-extension coupling matrix being zero is no longer valid due to the sandwich structure no longer being close to symmetric about its middle axis.

- Deflections due to Indentation at the cross head contact point are not accounted for in classical beam or plate theory, which results in smaller calculated midpsan deflections for face sheet thicknesses of 0.012 (1ply) or less.

These results show that it is recommended that in the design of honeycomb sandwich structures subjected to three point bending, core ribbon orientation must be considered when optimizing strength and stiffness.

Finite element analysis models were created to compare test results to numerical data to validate the experimental results. All beams were validated within an error of $17 \%$ for midspan deflections between experimental and numerical data. A potential source of error between experimental and numerical data could be due to inaccuracies in mechanical properties found experimentally and the experimental processes.

As composite sandwich structures become more widely used in engineering fields in the near future, the need for slight advantages in sandwich structure design will be crucial. Depending on design 
specifications, core orientation of honeycomb core sandwiches could be that slight advantage. As this report has shown, considering the core orientation within composite sandwiches, and for structures experiencing only static loading in one direction, increasing ply count on the face of applied force results in optimized designs of a structure and a better overall performance, which, in any industry, leads to an increase in money saved. 


\subsection{Future Considerations}

There have few studies into the behaviors of honeycomb core composite sandwich structures resulting in an increased safety factor in industries, which is against the ideal of optimization of weight reduction in design. This study delves into the behavior of honeycomb cores at different orientations but more can be learned about this material. The main idea behind design optimization, particularly in the aerospace industry, is to minimize weight while still obtaining the same, or improved, structural capabilities as typical materials found in industry, such as aluminum and steel. Further studies could include but are not limited to:

- Testing of the effects on the mechanical behavior of sandwich composites with different core materials, thickness's, densities, cell sizes and cell shapes coupled with the varying core ribbon orientations.

- Repeating this study under four point bends to verify face sheet to core thickness ratios for optimal bending stiffness and strength with respect to minimum weight

- Modelling specimens with the inclusion of plastic deformation of the indenter at the applied load, coupled with higher order beam theory. 


\section{References}

1. "Composite Materials." Composite Materials. The Aviation History On-Line Museum, 28 Mar. 2010. Web. 23 Jan. 2014.

2. Gay D., Hoa S,V., Tsai S,W. Composites Materials Design and Application. CRC Press

3. Analysis and Performance of Fiber Composites, $2^{\text {nd }}$ Ed., B.D. Agarwal and L.J. Broutman, Wiley, 1990, pp. 1-4.

4. Dr. Ray Franco, PhD., PE. "Bimetal Strips." Bimetal Strips. N.p., 2006. Web. 23 Jan. 2014.

5. "Arborite - High Pressure Laminates." Arborite. N.p., 2010. Web. 23 Jan. 2014.

6. Jones, R. Mechanics of Composite Materials $2^{\text {nd }}$ Edition.

7. Ferreira S., Lopez P., Monteiro S., Nascimento D., Natural-Fiber Polymer-Matrix Composites: Cheaper, Tougher, and Environmentally Friendly. JOM, a publication of the minerals, metals and materials society. January 2009.

8. Soutis C, Curtis PT. Fiber Reinforced Composites in Aircraft Construction. Aerospace Engineering, The University of Sheffield, Mappin Street, Sheffield S1 3JD, UK.

9. "Carbon Fiber Processing Part 1 - Wet / Hand Lay-up." Carbon Fiber Guru. N.p., 18 Jan. 2010. Web. 23 Jan. 2014.

10. Lee, S. Handbook of Composite Reinforcements. John Wiley \& Sons, Nov 30, 1992.

11. "What Is Vacuum Infusion Processing (VIP)." Structural Composite Designs. N.p., n.d. Web. 23 Jan. 2014.

12. "How Are Filament Winding Working?" Hebei Maple Fiberglass Industry Co., 2013. Web. 23 Jan. 2014.

13. Soutis C, Curtis PT. Prediction of the post-impact compressive strength of CFRP laminated composites. Comp Sci Technol 1996;56(6):677-84.

14. "Aluminum Honeycomb Panels." Honeycomb Panel, Aluminum Honeycomb Panel, Aluminum Honeycomb Core Manufacturers. Universal Metaltek, 2014. Web. 23 Jan. 2014.

15. Cross D, N. The Use of Honeycomb Sandwich Panels in the Engineering Field. Ciba-Geigy.

16. Nomex Honeycomb Data Sheet. Hexcel

17. Foo. C., Chai. G., Seah. L., Mechanical Properties of Nomex Material and Nomex Honeycomb Structure.

18. Walker C, H. Honeycomb Fastening. Fort Worth Div. General Dynamics Corp. Oct 5-9, 1970.

19. Petras, A. Design of Sandwich Structures. Robinson College, Cambridge (1998)

20. Chesser, Paul. "Technical Glitches and Payments for Down Time Still Nag Boeing's Dreamliner." Somewhat Reasonable. N.p., 10 Aug. 2013. Web. 23 Jan. 2014.

21. Norris, Guy (January 9, 2009). "Boeing Rules Out 787 Window Change”. Aviation Week. Retrieved June 14, 2013.

22. Baruch. M , Frostig. Y., Sheinman. I., Vilnay., O. Bending of Nonsymmetric Sandwich Beams with Transversely Flexible Core. Journal of Engineering Mechanics. September 1991.

23. Lim, T.S., Lee, C.S. and Lee. D.G. Failure Modes of Foam Core Sandwich Beams under Static and Impact Loads. Mechanical Design Laboratory with Advanced Materials, Department of Mechanical Engineering, ME3261, Korea Advanced Institute of Science and Technology, 373-1, Guseong-dong, Yuseong-gu, Daejeon-shi, 305-701, Korea 
24. Khan, Subhotosh. Bonding of Sandwich Structures - The Facesheet/Honeycomb Interface. E.I. DuPont Nemours Co., Inc. Advanced Fibers System, Richmondm VA 23234. Jan 25, 2007.

25. Allen, H. G., Analysis and Design of Structural Sandwich Panels. Oxford New York, Pergamon, (1969).

26. Triantafillou, T.C. and Gibson, L.J. (1987). Failure Mode Maps for Foam Core Sandwich Beams, Materials Science and Engineering, 95: 37-53.

27. Plantema, F. J. Sandwich Construction, John Wiley and Sons, New York (1966)

28. Ashby M. F., Gibson, L.J., Cellular Solids: structure and Properties. Pergamon Press, Oxford, 1988.

29. Gibson, L. J., Triantafillou, T. C. Minimum Weight Design of Foam Core Sandwich Panels for a Given Strength. Materials Science and Engineering, 95, 1987, pp.55-62.

30. ASTM D3039; Standard Test Method for Tensile Properties of Polymer Matrix Composite Materials.

31. ASTM E132; Standard Test Method for Poisson's Ratio at Room Temperature.

32. ASTM D6641; Standard Test Method for Compressive Properties of Polymer Matrix Composite Materials Using a Combined Loading Compression (CLC) Test Fixture.

33. ASTM D3518; Standard Test Method for In-Plane Shear Response of Polymer Matrix Composite Materials by Tensile Test of $\mathrm{a}+/-45^{\circ}$ Laminate

34. ASTM D2584; Standard Test Method for Ignition Loss of Cured Reinforced Resins

35. 3M Scotch-Weld Structural Adhesive Film AF 163-2 Technical Datasheet.

36. ASTM D638; Standard Test Method for Tensile Properties of Plastics

37. ASTM C273; Standard Test Method for Shear Properties of Sandwich Core Materials

38. ASTM D6790; Standard Test Method for Determining Poisson's Ratio of Honeycomb Cores

39. ASTM C 393; Standard Test Method for Flexural Properties of Sandwich Constructions.

40. ASTM D7249; Standard Test Method for Facing Properties of Sandwich Constructions by Long Beam Flexure

41. ASTM D7250; Standard Practice for Determining Sandwich Beam Flexural and Shear Stiffness

42. American Society for Testing and Materials Annual Book of ASTM Standards, (2000).

43. Tae Seong Lim, Chang Sup Lee, Dai Gil Lee. "Failure Modes of Foam Core Sandwich Beams under Static and Impact Loads". Journal of Composite Materials. Sage Publications. August 27, 2004.

44. Whitney, J. A Local Model for Bending of Weak Core Sandwich Plates. Journal of sandwich structures and Materials 2001 3: 269. Oct 1, 2001.

45. T.R.A.Pearce, The stability of Simply-Supported Sandwich Panels with Fiber Reinforced Face Plates, Ph.D. thesis, University of Bristol, U.K., 1973.

46. Selvadurai, A. Elastic Analysis of soil - foundation interaction, Developments in Geotechincal Engineering, 17, 1979.

47. Froud, G. R. (1980). Your Sandwich Order, Sir, Composites, 11(3): 133-138.

48. Johnson, A. F. and Sims, G. D. (1986). Mechanical Properties and Design of Sandwich Materials, Composites,17(4): 321-328. 
Appendices

A1. Mechanical Properties

A.1.1 Tensile tests of Hexply 6376 AGP-250H

\begin{tabular}{|c|c|c|c|c|}
\hline & Specimen label & $\begin{array}{c}\text { Maximum Load } \\
\text { (lbf) }\end{array}$ & $\begin{array}{l}\text { Maximum Tensile } \\
\text { stress } \\
\text { (ksi) }\end{array}$ & $\begin{array}{l}\text { Extension at } \\
\text { Maximum Load } \\
\text { (in) }\end{array}$ \\
\hline 1 & 1 & 5391.79915 & 78.37032 & 0.05786 \\
\hline 2 & 2 & 5721.65313 & 83.15148 & 0.05901 \\
\hline 3 & 3 & 6248.14169 & 92.97830 & 0.06458 \\
\hline 4 & 4 & 5475.95435 & 86.82894 & 0.06404 \\
\hline 5 & 5 & 6017.21470 & 89.77702 & 0.06353 \\
\hline 6 & 6 & 5688.56670 & 82.85487 & 0.05999 \\
\hline 7 & 7 & 5433.49352 & 80.30228 & 0.05736 \\
\hline Mean & & 5710.97475 & 84.89474 & 0.06091 \\
\hline $\begin{array}{l}\text { Standard } \\
\text { Deviation }\end{array}$ & & 320.68958 & 5.22463 & 0.00307 \\
\hline $\begin{array}{l}\text { Coefficien } \\
\text { t of } \\
\text { Variation }\end{array}$ & & 5.61532 & 6.15424 & 5.03561 \\
\hline $\begin{array}{l}\text { Mean + } 1 \\
\text { SD }\end{array}$ & & 6031.66433 & 90.11937 & 0.06398 \\
\hline $\begin{array}{l}\text { Mean - } 1 \\
\text { SD }\end{array}$ & & 5390.28517 & 79.67012 & 0.05784 \\
\hline
\end{tabular}




\begin{tabular}{|c|c|c|c|c|}
\hline & $\begin{array}{c}\text { Modulus (Automatic } \\
\text { Young's) } \\
\text { (ksi) }\end{array}$ & $\begin{array}{l}\text { Length } \\
\text { (in) }\end{array}$ & $\begin{array}{l}\text { Thickness } \\
\text { (in) }\end{array}$ & $\begin{array}{l}\text { Width } \\
\text { (in) }\end{array}$ \\
\hline 1 & 12510.26100 & 8.00000 & 0.07100 & 0.96900 \\
\hline 2 & 12853.71141 & 8.00000 & 0.07000 & 0.98300 \\
\hline 3 & 13135.08604 & 8.00000 & 0.07000 & 0.96000 \\
\hline 4 & 13281.96035 & 8.00000 & 0.06900 & 0.91400 \\
\hline 5 & 12062.47697 & 8.00000 & 0.07100 & 0.94400 \\
\hline 6 & 12734.32547 & 8.00000 & 0.07100 & 0.96700 \\
\hline 7 & 12801.96279 & 8.00000 & 0.07100 & 0.95300 \\
\hline Mean & 12911.39772 & 8.00000 & 0.07043 & 0.95571 \\
\hline $\begin{array}{l}\text { Standard } \\
\text { Deviation }\end{array}$ & 263.86456 & 0.00000 & 0.00079 & 0.02218 \\
\hline $\begin{array}{l}\text { Coefficien } \\
\text { t of } \\
\text { Variation }\end{array}$ & 2.21523 & 0.00000 & 1.11715 & 2.32066 \\
\hline $\begin{array}{l}\text { Mean + } 1 \\
\text { SD }\end{array}$ & 12175.26228 & 8.00000 & 0.07122 & 0.97789 \\
\hline $\begin{array}{l}\text { Mean - } 1 \\
\text { SD }\end{array}$ & 11647.53316 & 8.00000 & 0.06964 & 0.93354 \\
\hline
\end{tabular}

\begin{tabular}{|r|c|c|c|}
\hline & $\begin{array}{c}\text { Area } \\
(\text { in^2) }\end{array}$ & $\begin{array}{c}\text { Tensile stress at } \\
\text { Maximum Load } \\
(\mathrm{ksi})\end{array}$ & $\begin{array}{c}\text { Tensile strain at } \\
\text { Maximum Load }\end{array}$ \\
\hline 1 & 0.06880 & 165.37032 & 0.00723 \\
\hline 2 & 0.06881 & 162.15148 & 0.00738 \\
\hline 3 & 0.06720 & 167.97830 & 0.00807 \\
\hline
\end{tabular}




\begin{tabular}{|r|c|c|c|}
\hline & Area & $\begin{array}{c}\text { Tensile stress at } \\
\text { Maximum Load }\end{array}$ & $\begin{array}{c}\text { Tensile strain at } \\
\text { Maximum Load }\end{array}$ \\
\hline 4 & 0.06307 & (ksi) & (in/in) \\
\hline 5 & 0.06702 & 159.82894 & 0.00800 \\
\hline 6 & 0.06866 & 165.85487 & 0.00793 \\
\hline 7 & 0.06766 & 163.30228 & 0.00750 \\
\hline $\begin{array}{l}\text { Mean } \\
\text { (in^) }\end{array}$ & 0.06732 & 163.89474 & 0.00716 \\
\hline $\begin{array}{l}\text { Standard } \\
\text { Deviation }\end{array}$ & 0.00202 & 5.22463 & 0.00038 \\
\hline $\begin{array}{l}\text { Coefficien } \\
\text { t of } \\
\text { Variation }\end{array}$ & 3.00249 & 6.15424 & 0.007618 \\
\hline $\begin{array}{l}\text { Mean + 1 } \\
\text { SD }\end{array}$ & 0.06934 & 90.11937 & 0.00799 \\
\hline $\begin{array}{l}\text { Mean - 1 } \\
\text { SD }\end{array}$ & 0.06530 & & \\
\hline
\end{tabular}




\section{Specimen 1 to 8}

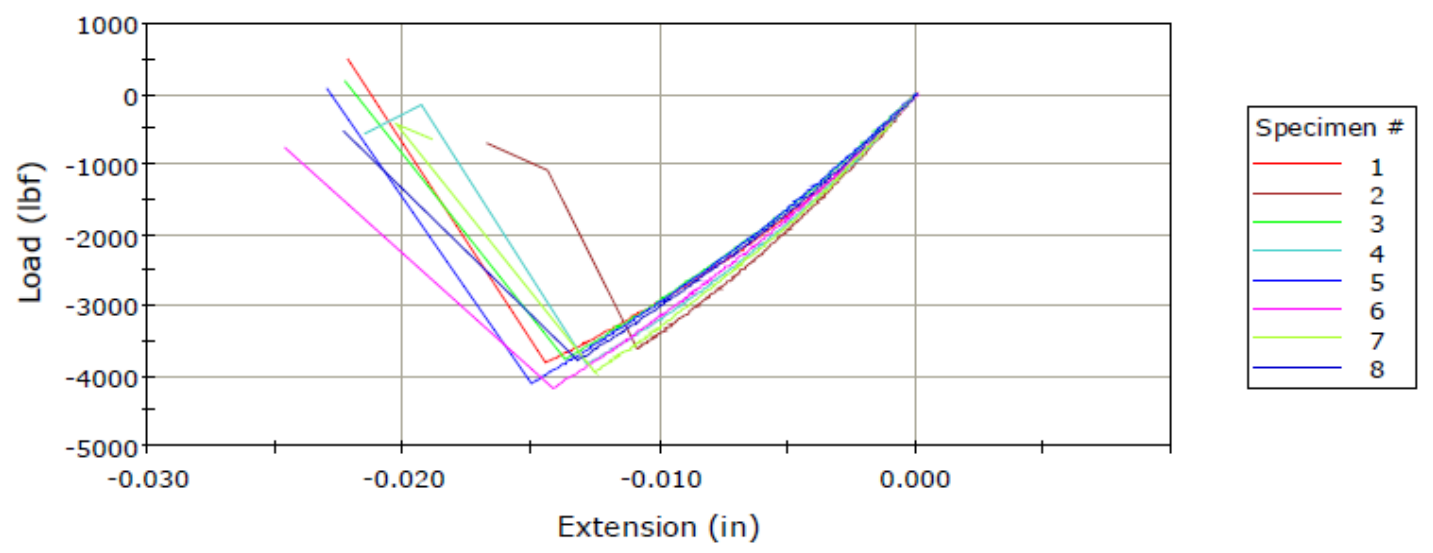

\begin{tabular}{|c|c|c|}
\hline & $\begin{array}{c}\text { Tensile } \\
\text { Strength } \\
(\mathrm{psi})\end{array}$ & Modulus \\
\hline & 128640.1 & (psi) \\
\hline & 124878.5 & 4125682 \\
\hline & 125167.9 & 4956487 \\
\hline & 129528.1 & 3932457 \\
\hline & 125776.4 & 5282718 \\
\hline & 125703.2 & 3912480 \\
\hline Mean & 134373.2 & 4715906 \\
\hline std & 127723.9 & 4484488 \\
\hline & 3435.438 & 4487174 \\
\hline
\end{tabular}




\section{A.2 Core Orientation Load and stress plots}

Figure 2.1.A. \& 2.2.A. 0 Degree Core Load and Facing Stress Curves - Longitudinal Core Direction
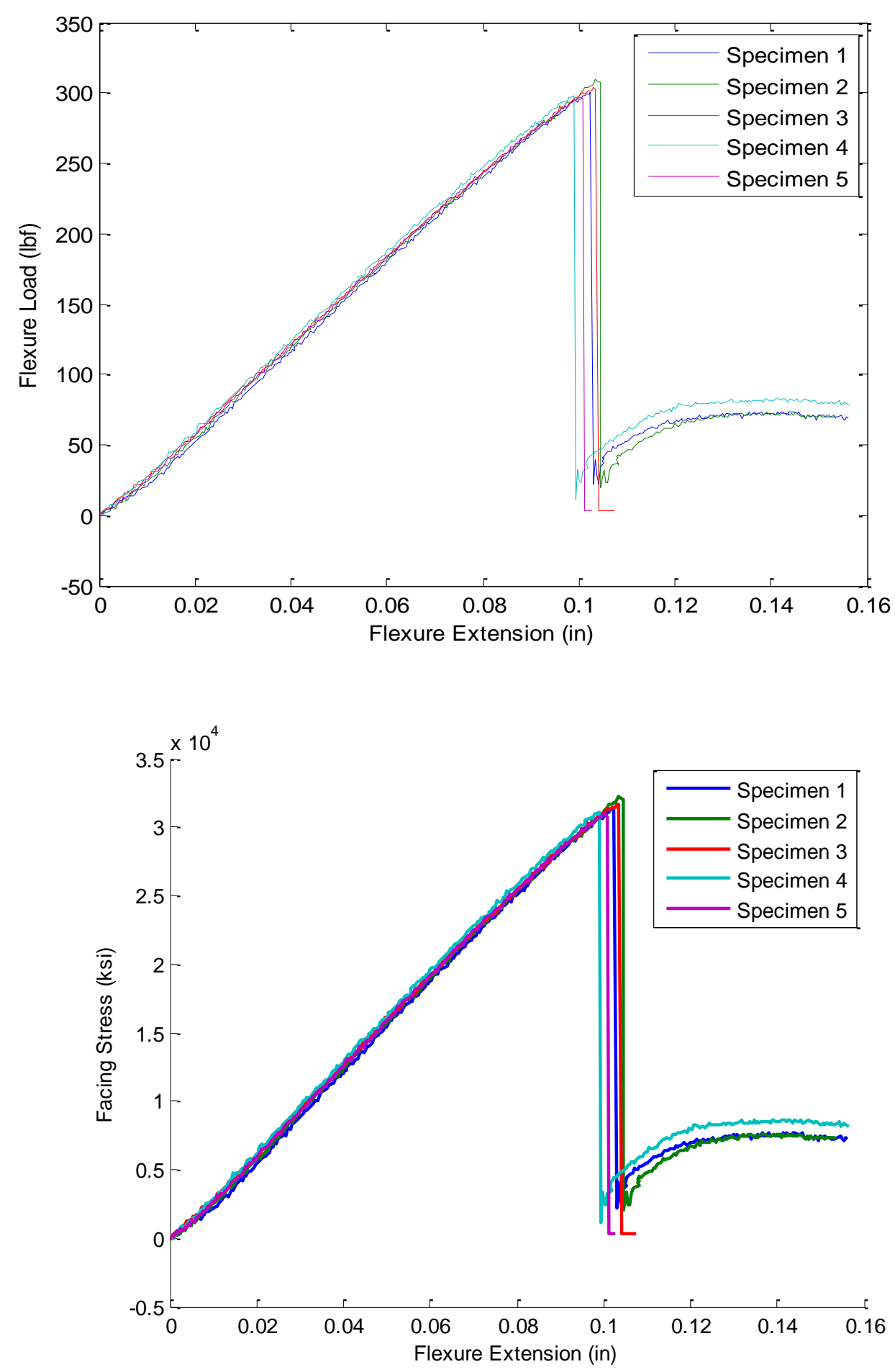
Figures 2.3.A \& 2.4.A. 15 Degree - Longitudinal Core Direction
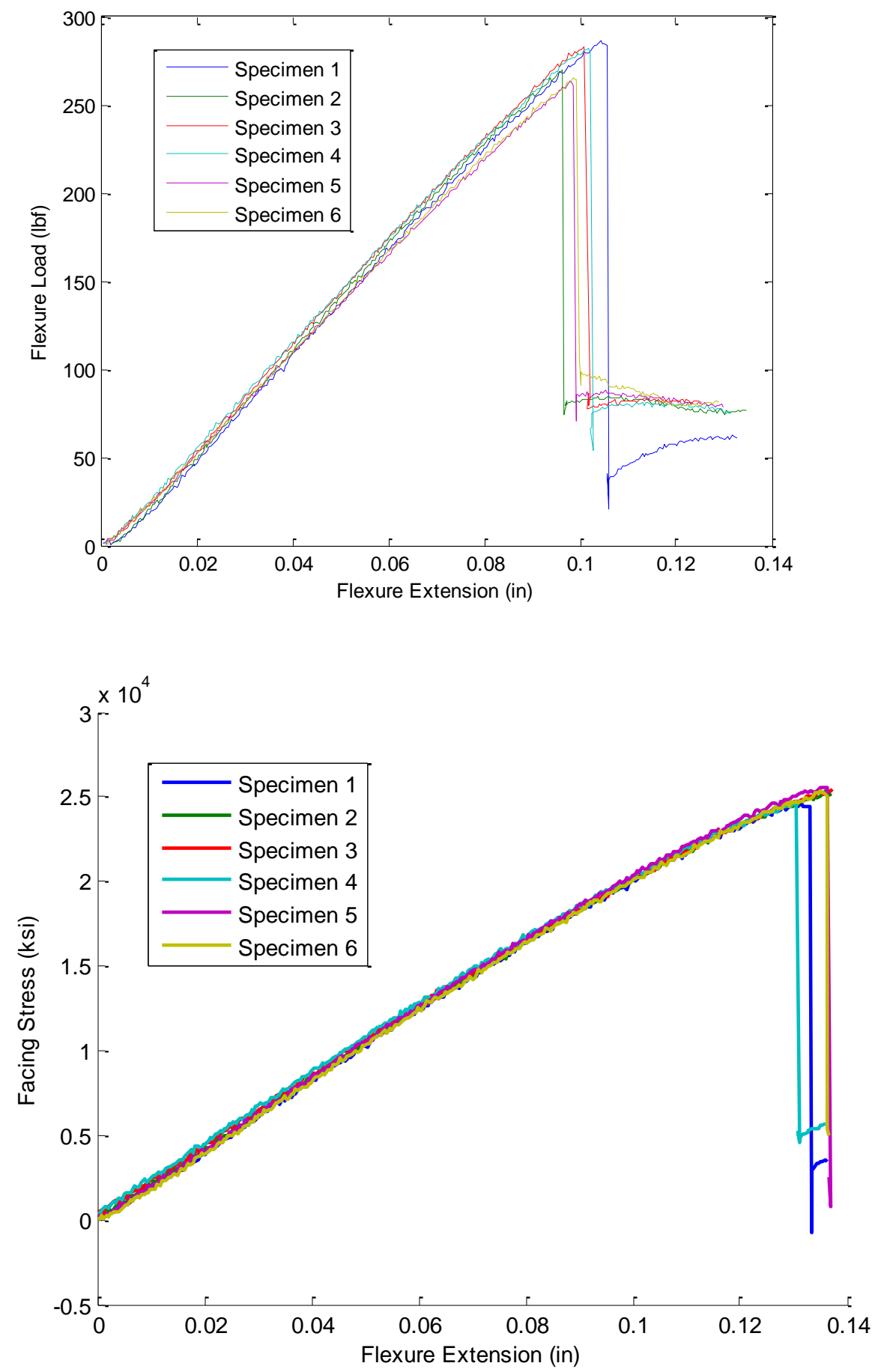
Figure 2.5.A. \& 2.6.A. 30-Degree Core Direction
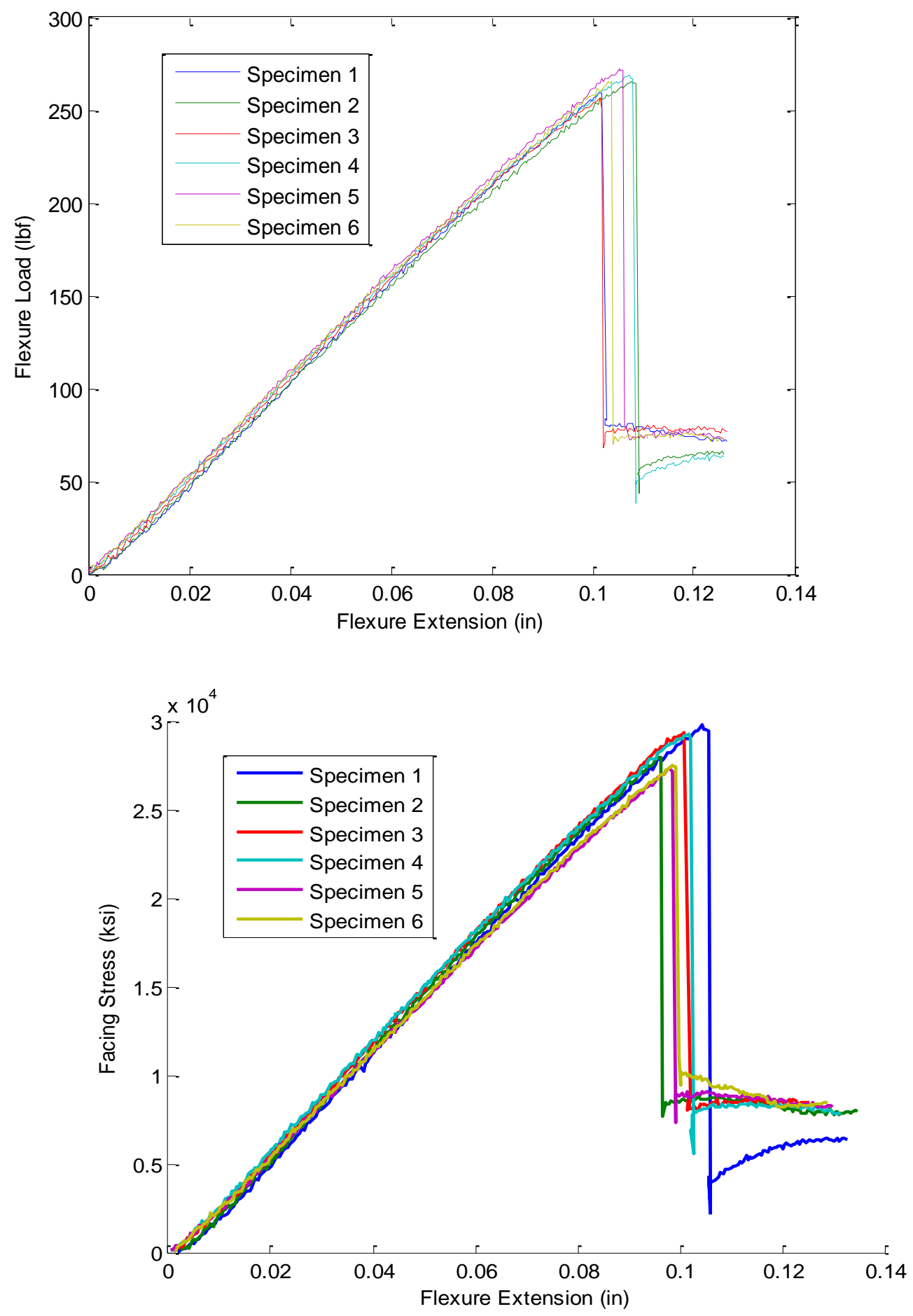
Figures 2.7.A. \& 2.8.A. 45-Degree Core Direction
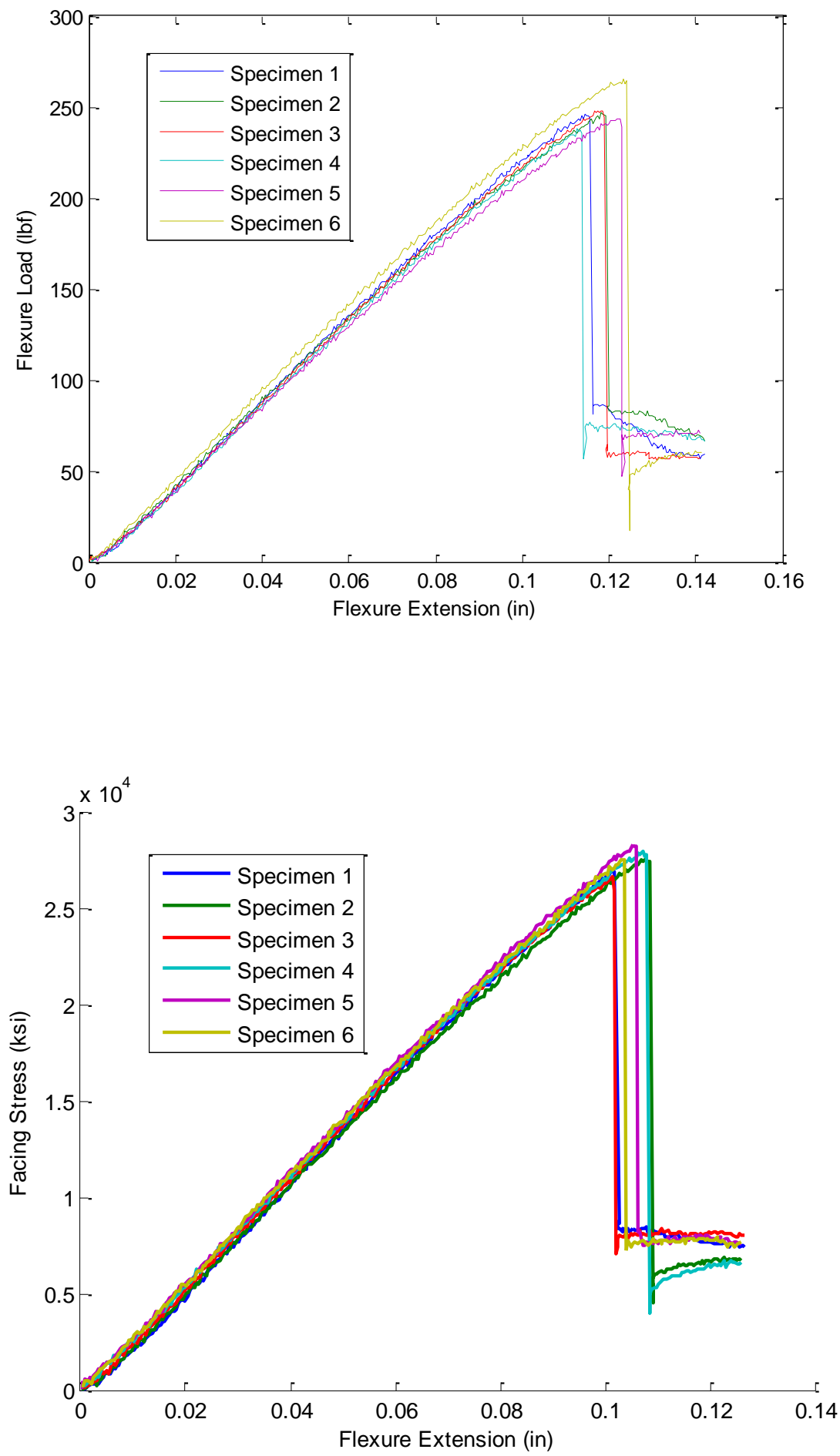
Figures 2.9.A. \& 2.10.A. 60-Degree Core Direction
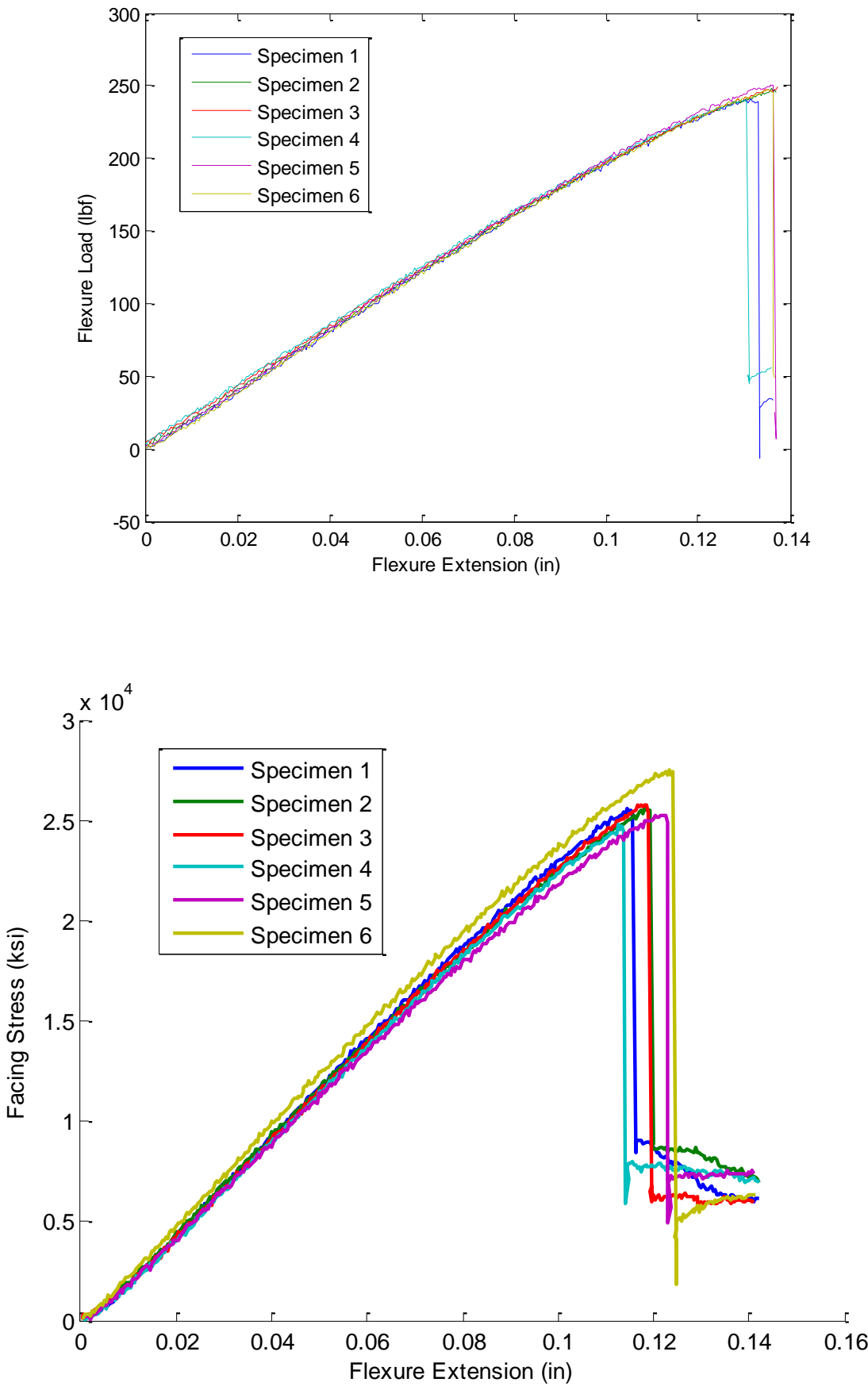
Figures 2.11.A. \& 2.12.A. 75-Degree Core Direction
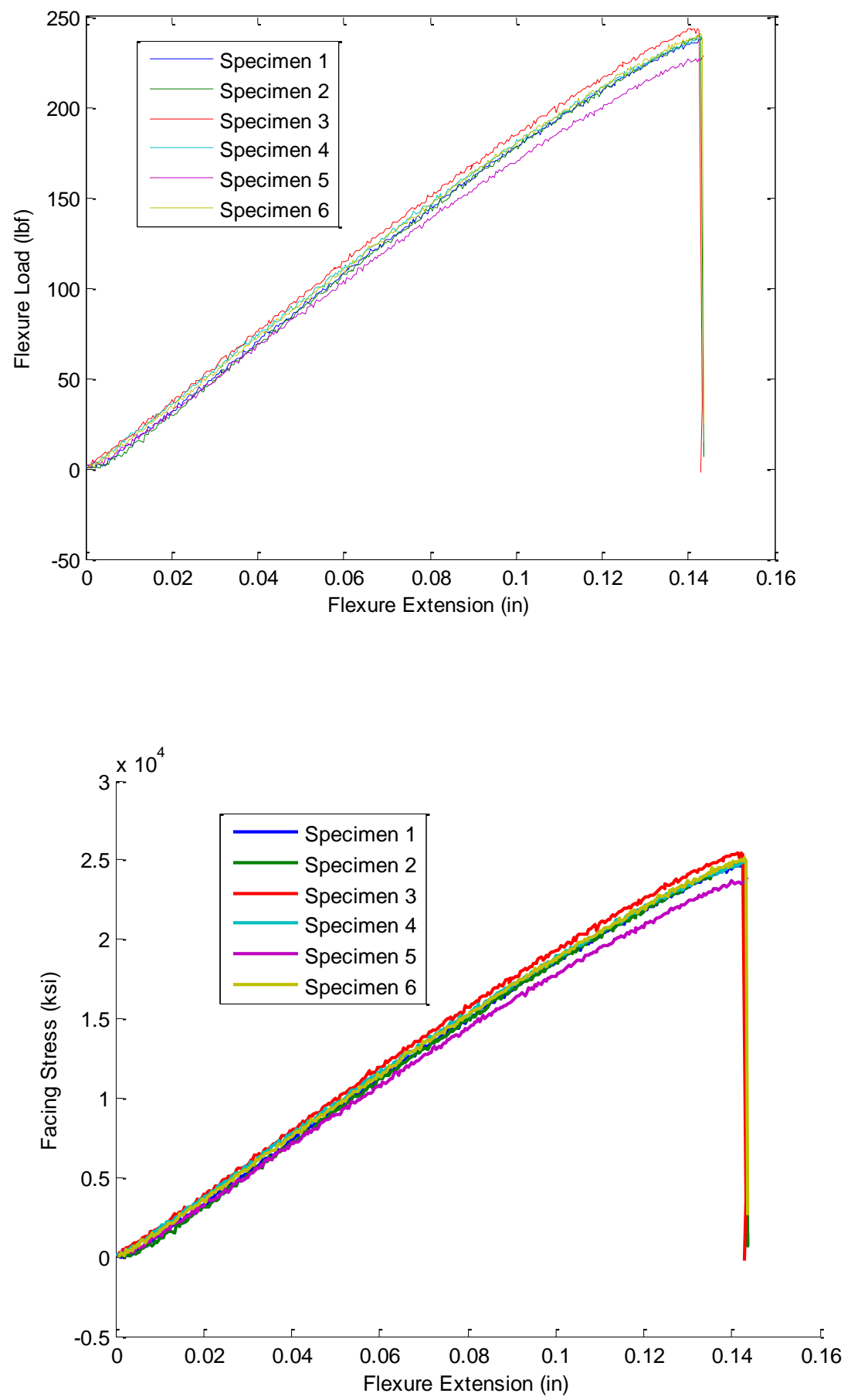
Figures 2.13.A. \& 2.14.A. 90-Degree Core Direction
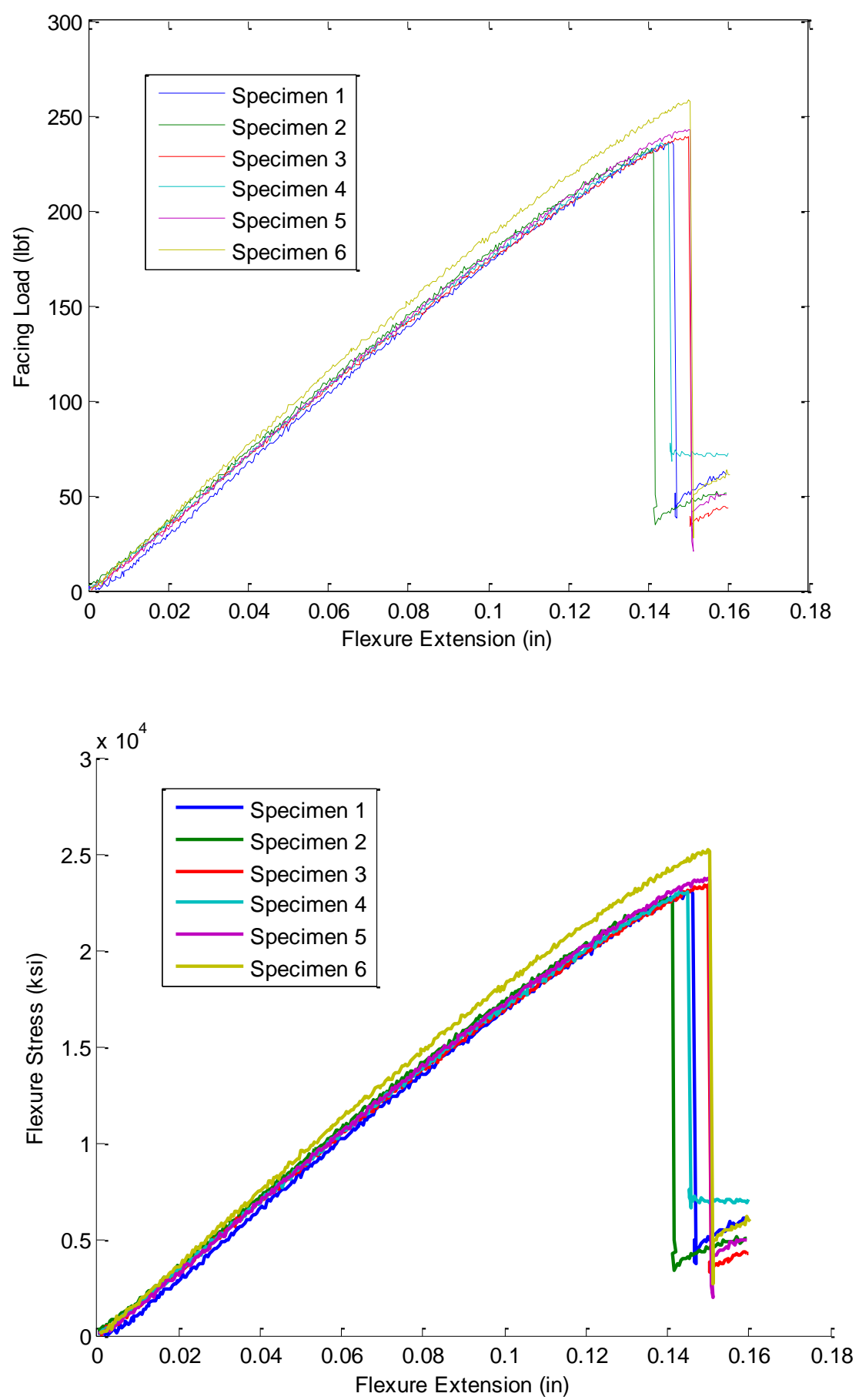


\section{A.3 Varying Face Sheet Thickness Load and Stress Plots}

* Bold Lines in stress plots Indicate bottom face sheet stress.

Figures 3.1.A. \& 3.2.A. 1/1 Configuration Load and Facing stress vs Extension Plots
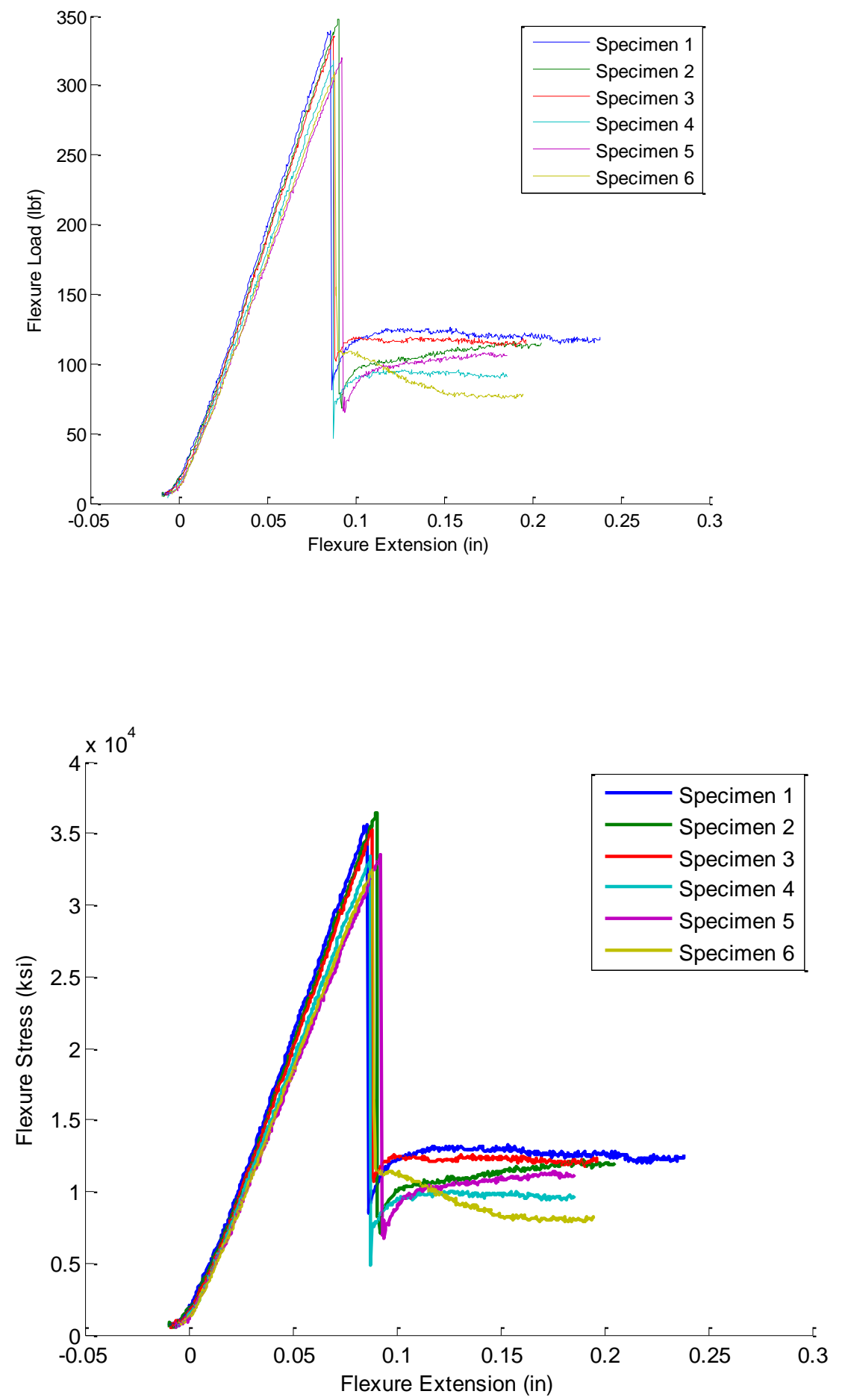
Figures 3.3.A. \& 3.4.A. 2/1 Configuration Load and Facing stress vs Extension Plots
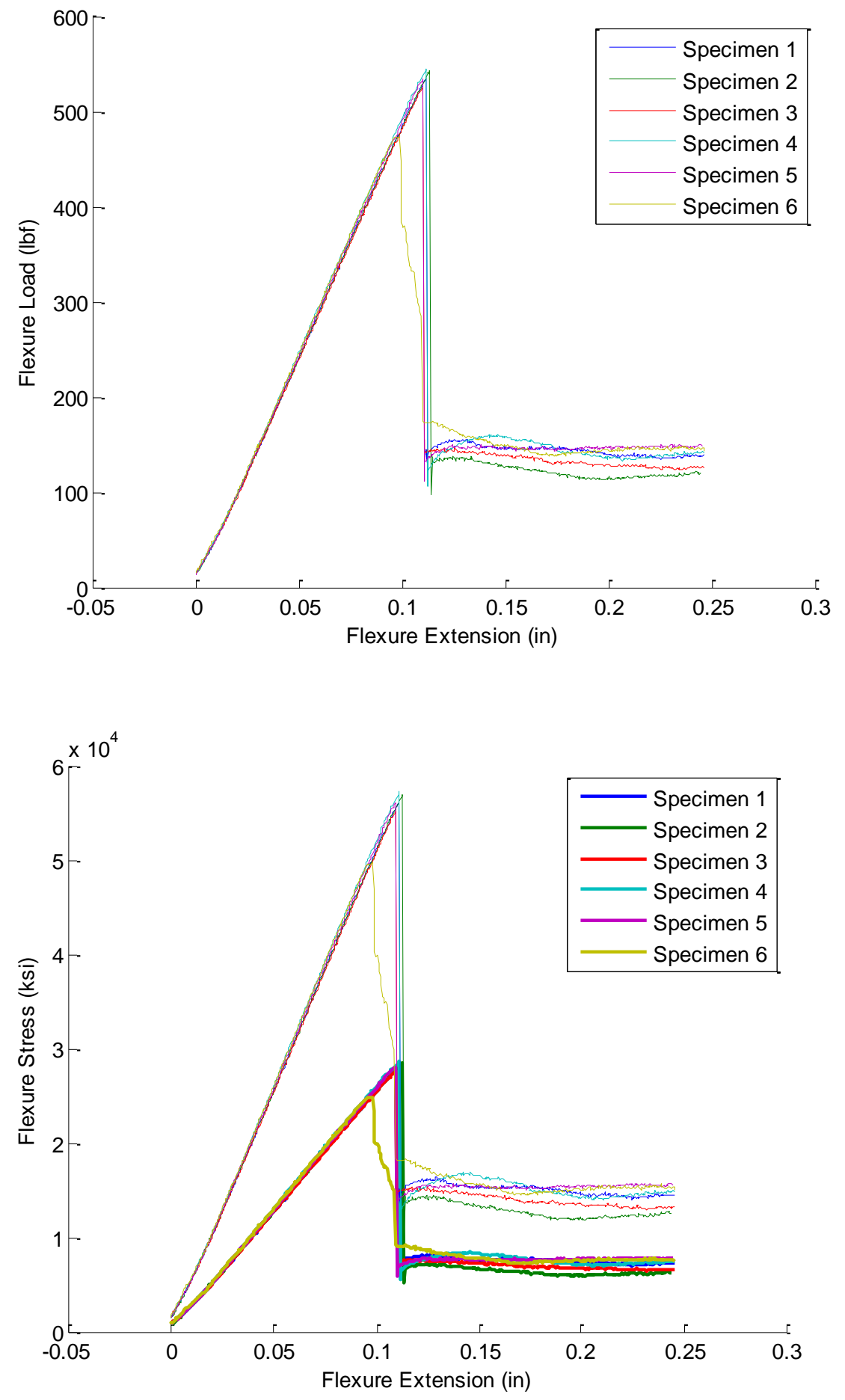
Figures 3.5.A. \& 3.6.A. 3/1 Configuration Load and Facing stress vs Extension Plots
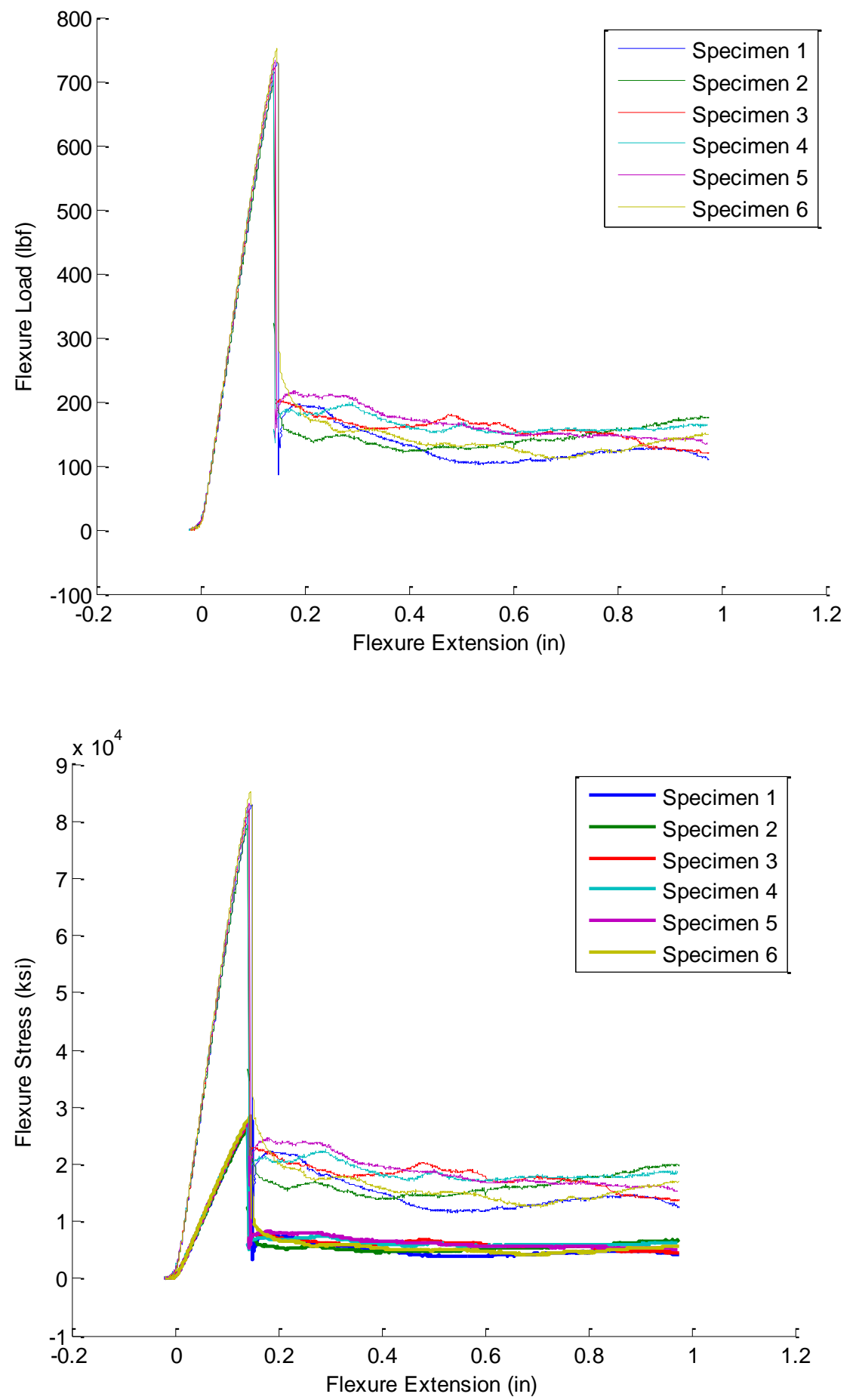
Figures 3.7.A. \& 3.8.A. 4/1 Configuration Load and Facing stress vs Extension Plots
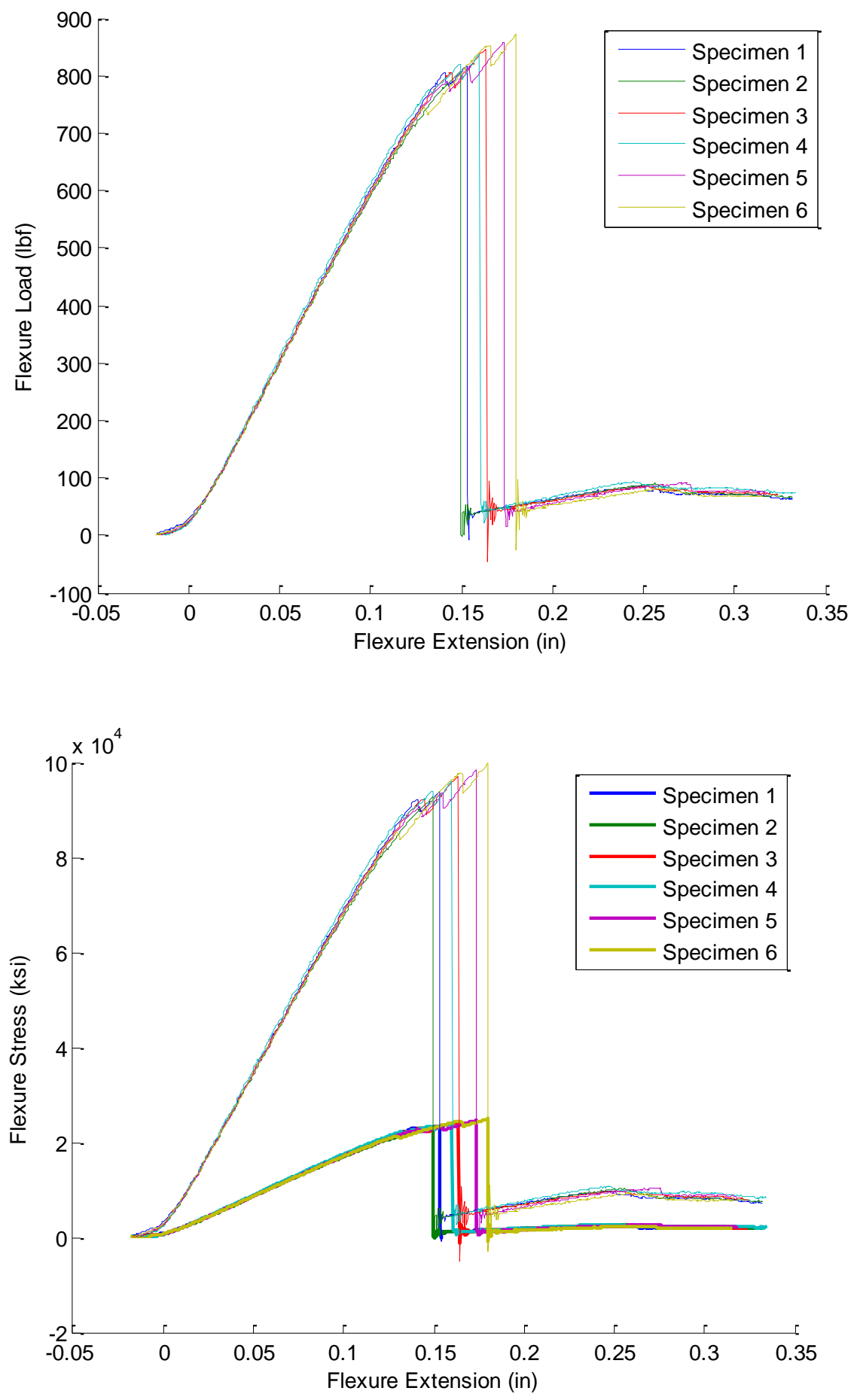
Figures 3.9.A. \& 3.10.A. 5/1 Configuration Load and Facing stress vs Extension Plots
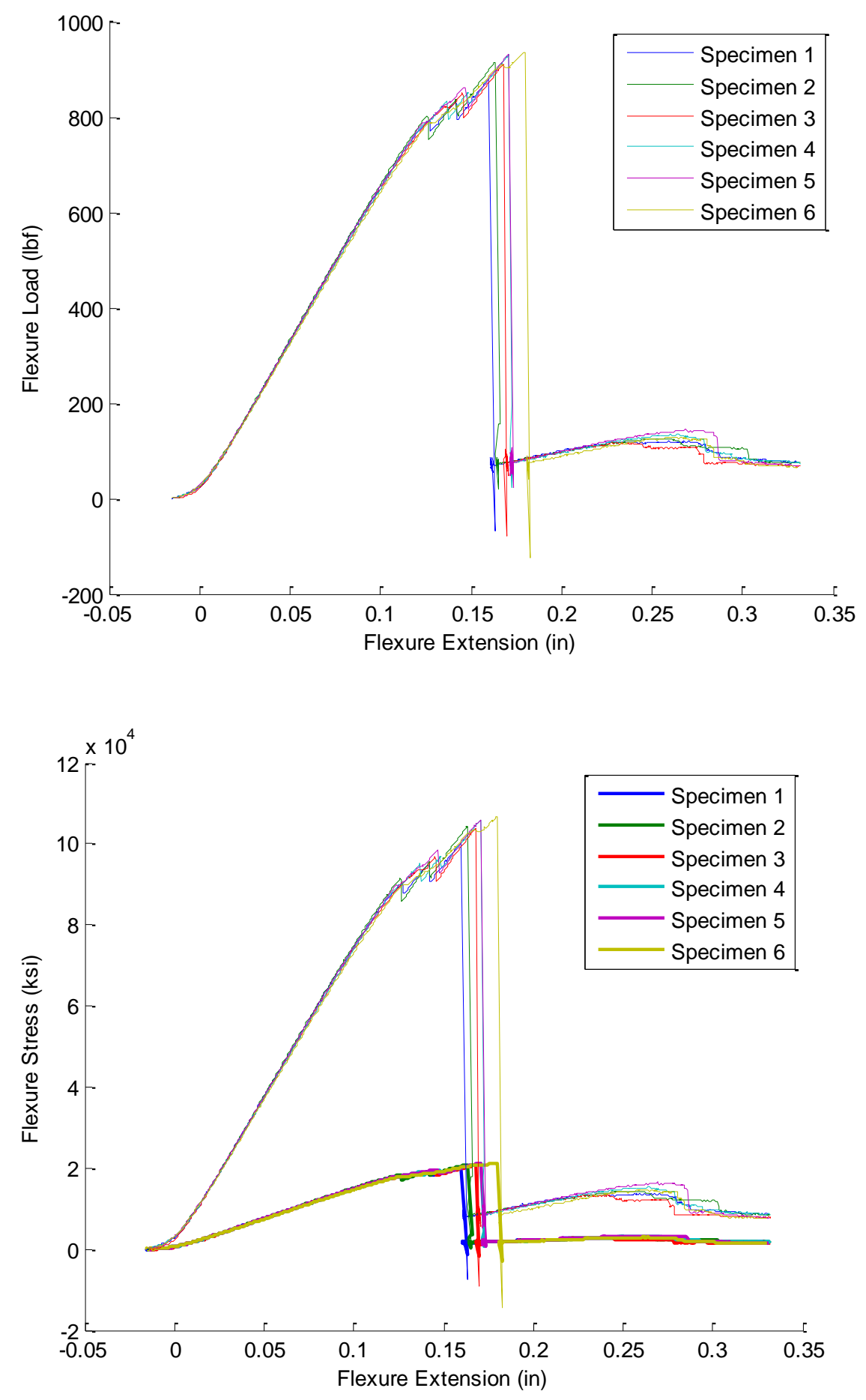
Figures 3.11.A. \& 3.12.A. 1/2 Configuration Load and Facing stress vs Extension Plots
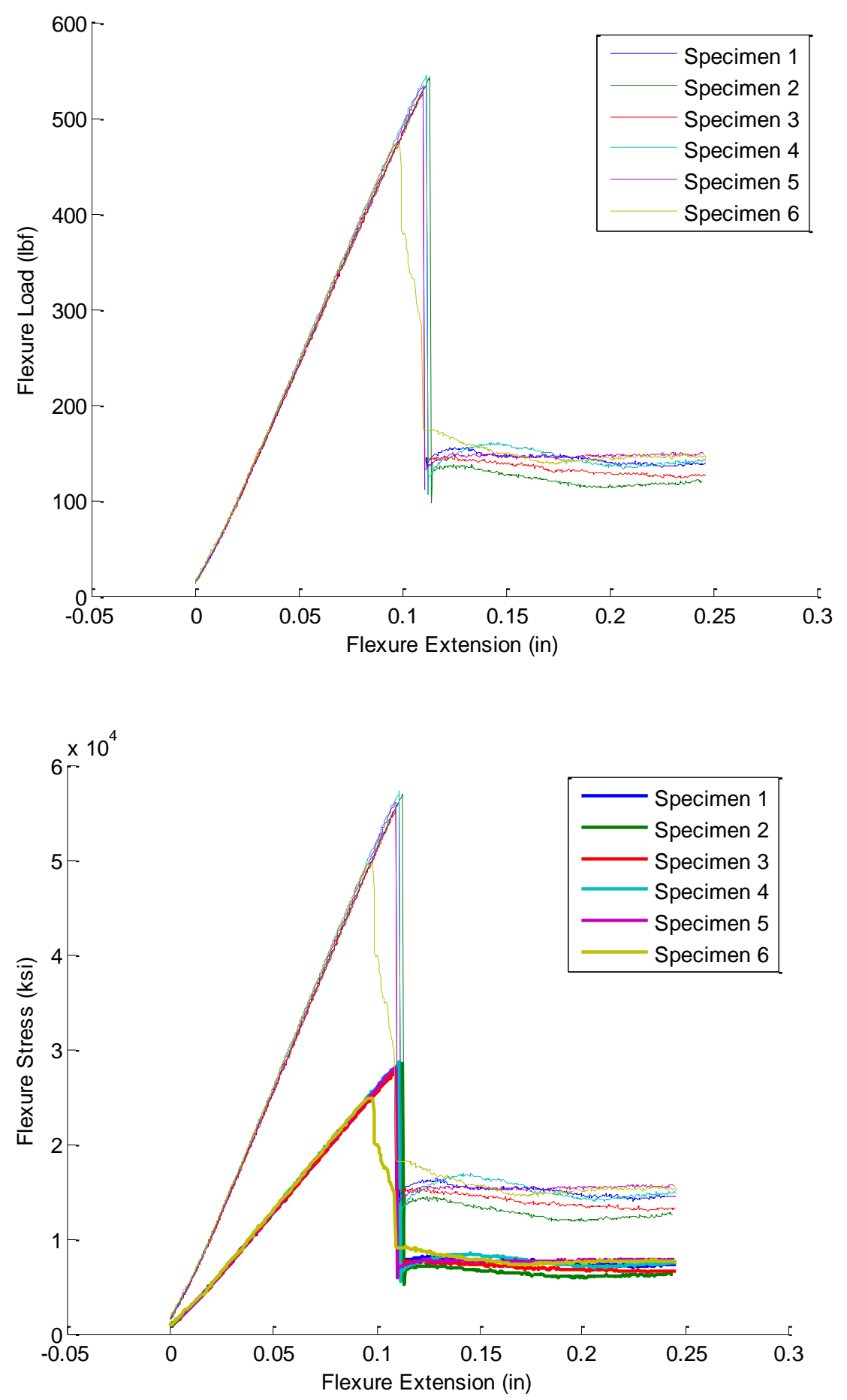
Figures 3.13.A. \& 3.14.A. 2/2 Configuration Load and Facing stress vs Extension Plots
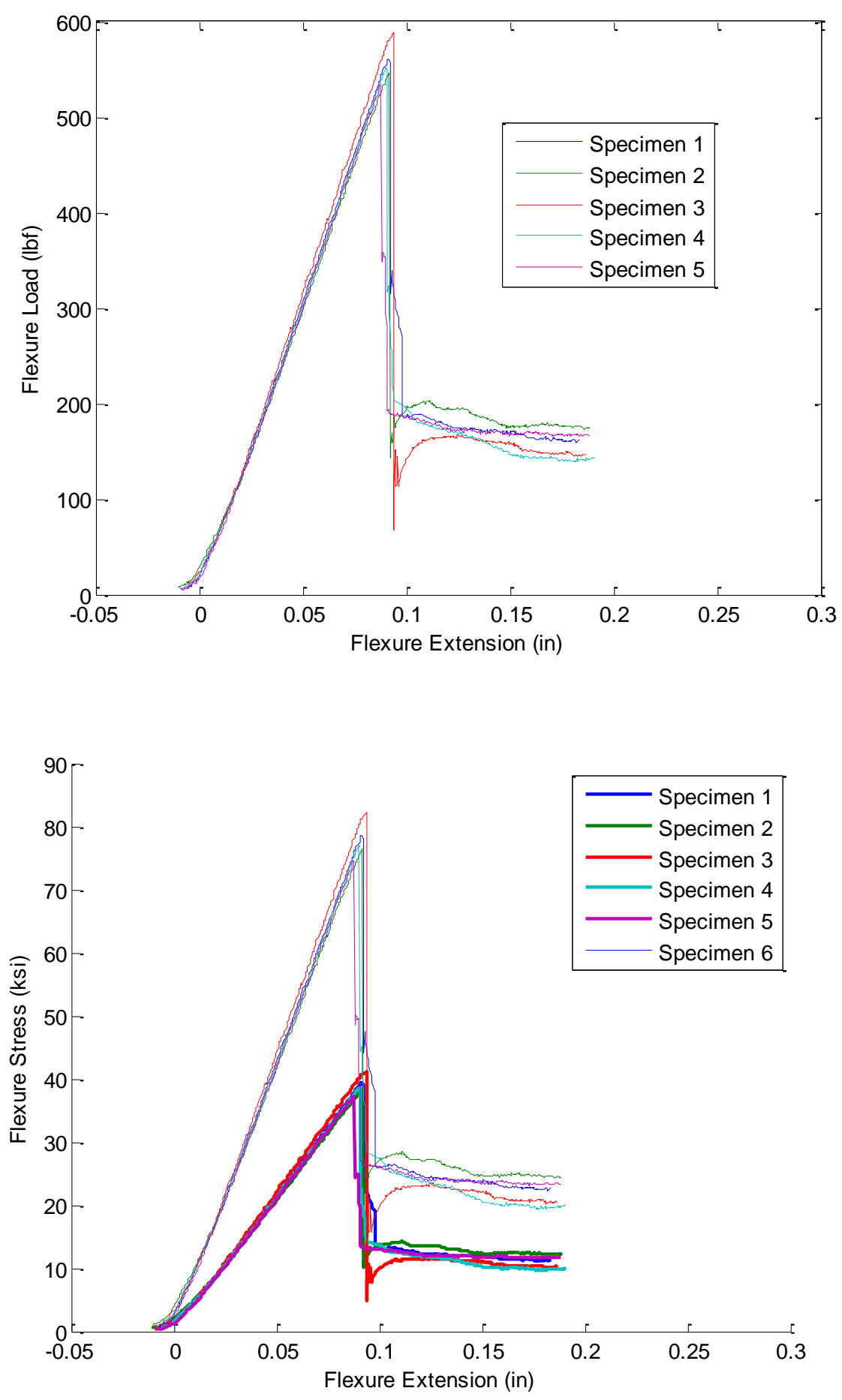
Figures 3.5.A. \& 3.16.A. 3/2 Configuration Load and Facing stress vs Extension Plots
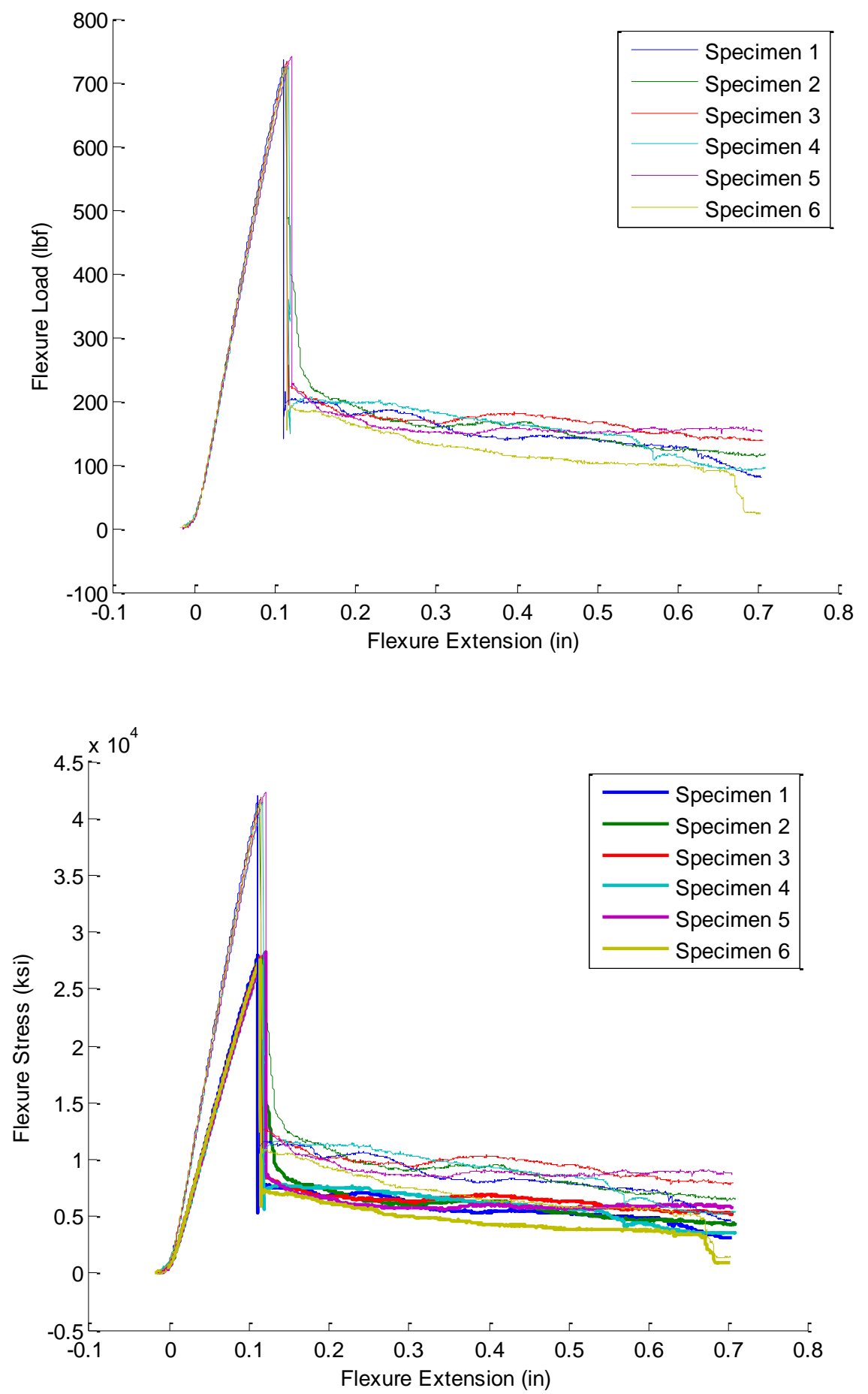
Figures 3.17.A. \& 3.18.A. 4/2 Configuration Load and Facing stress vs Extension Plots
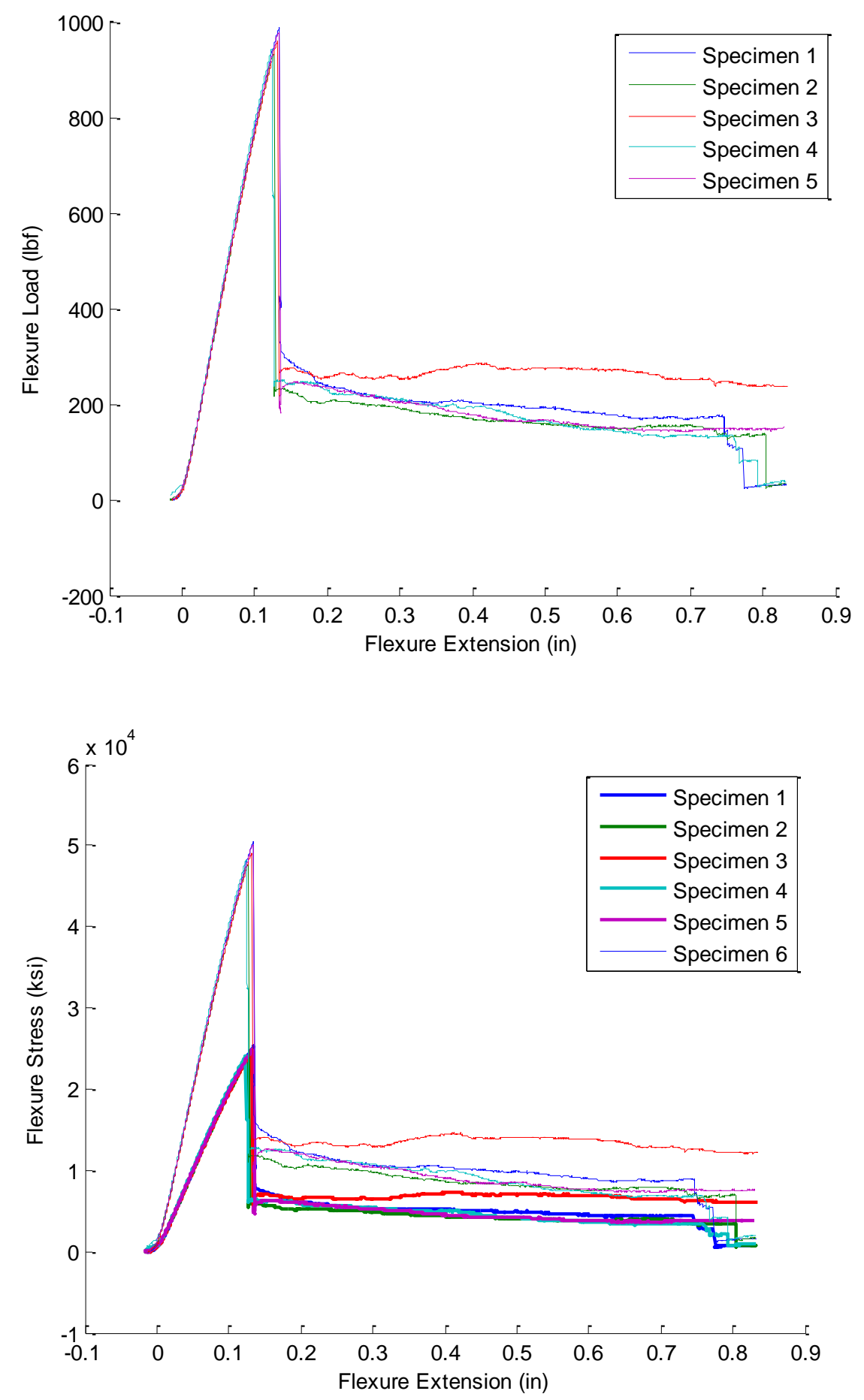
Figures 3.19.A. \& 3.20.A. 5/2 Configuration Load and Facing stress vs Extension Plots
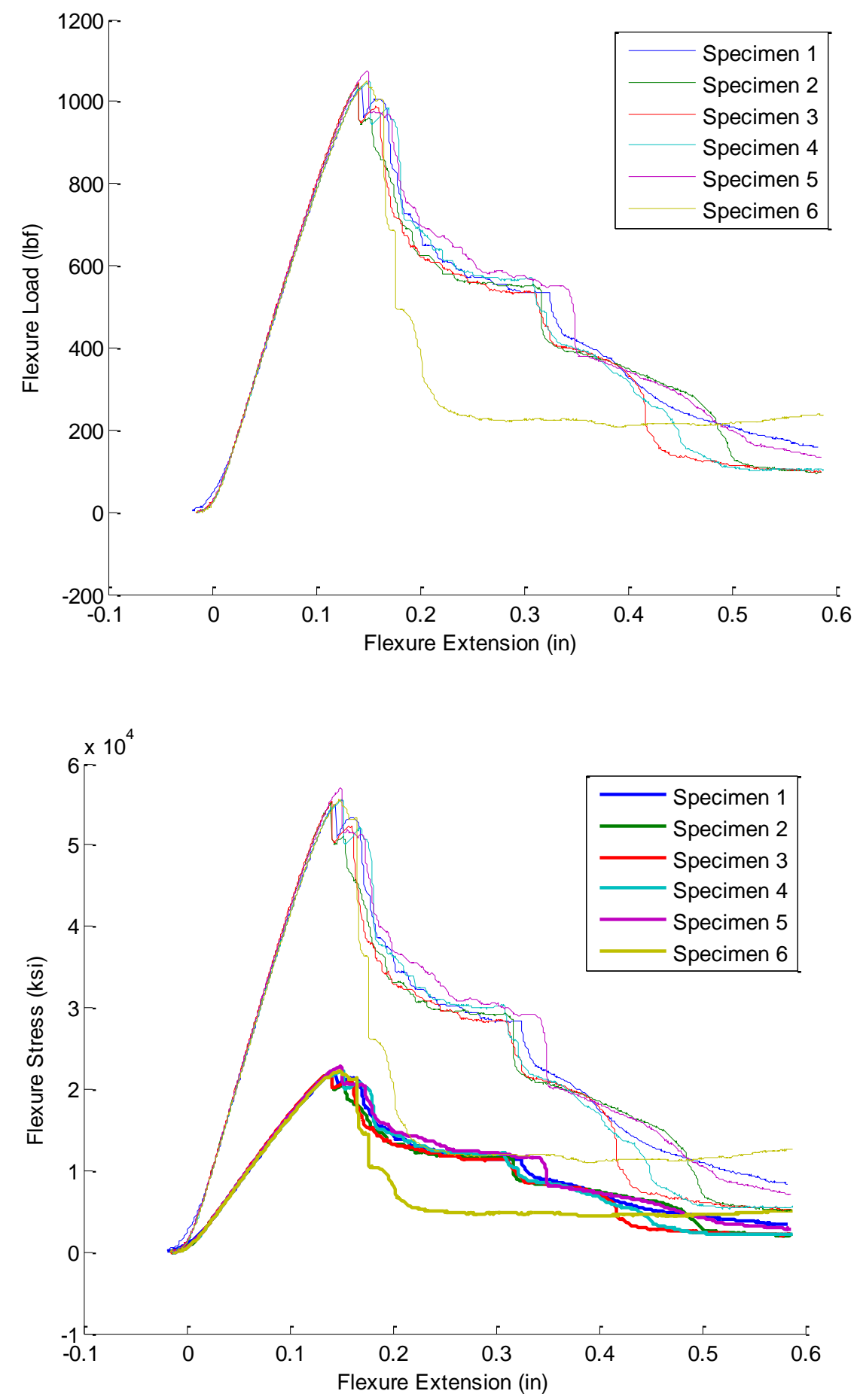
Figures 3.21.A. \& 3.22.A. 1/3 Configuration Load and Facing stress vs Extension Plots
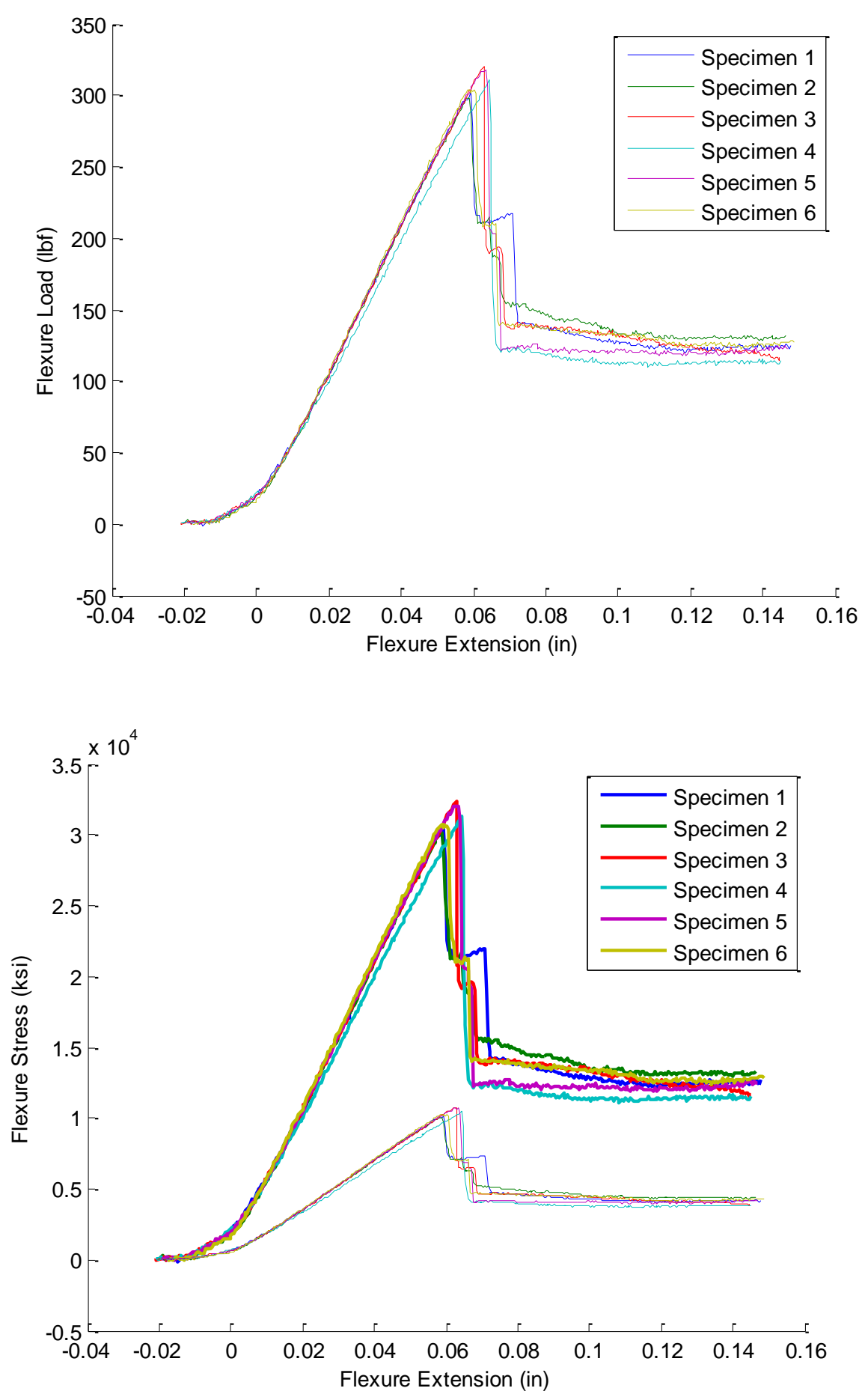
Figures 3.23.A. \& 3.24.A. 2/3 Configuration Load and Facing stress vs Extension Plots
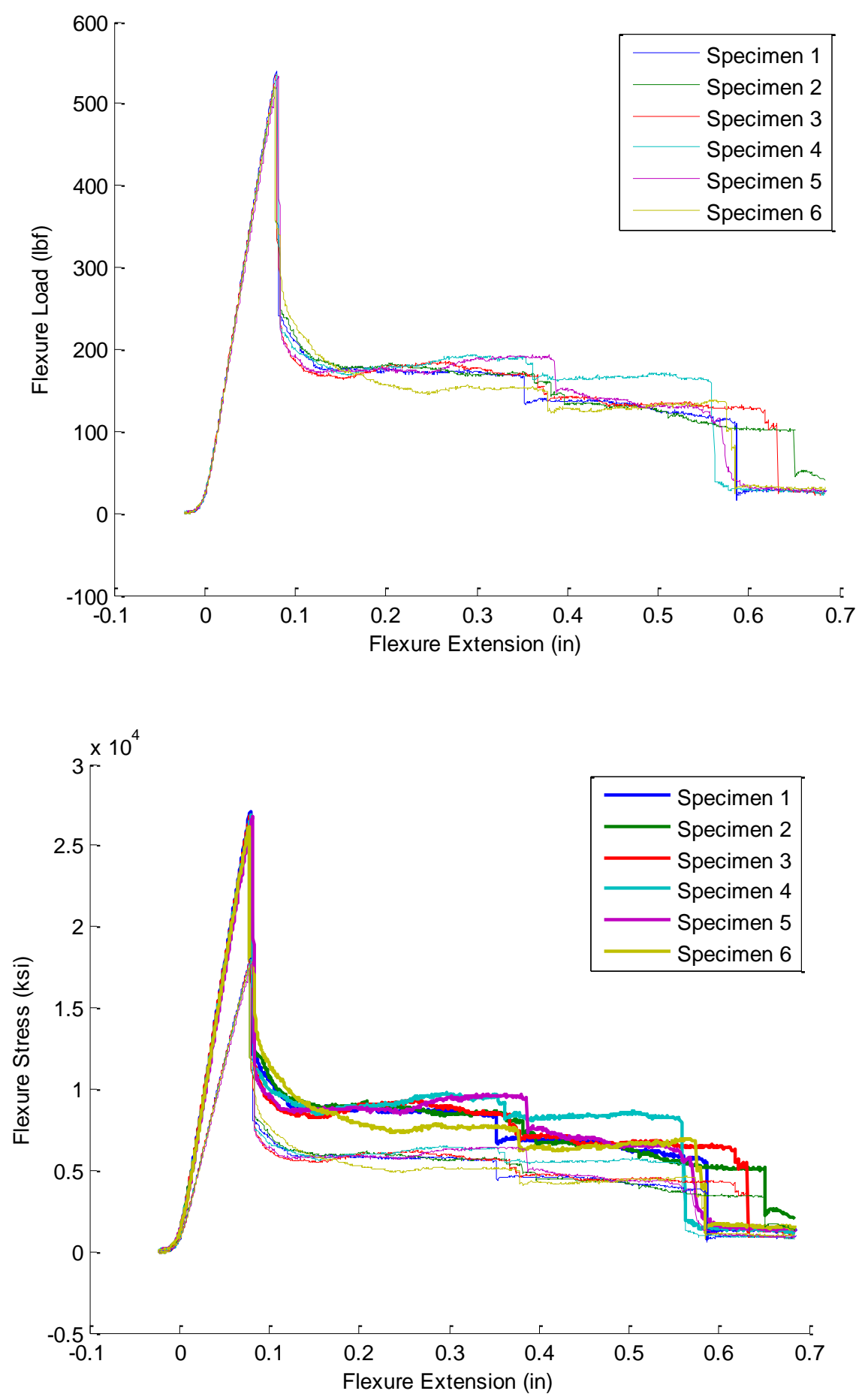
Figures 3.25.A. \& 3.26.A. 3/3 Configuration Load and Facing stress vs Extension Plots
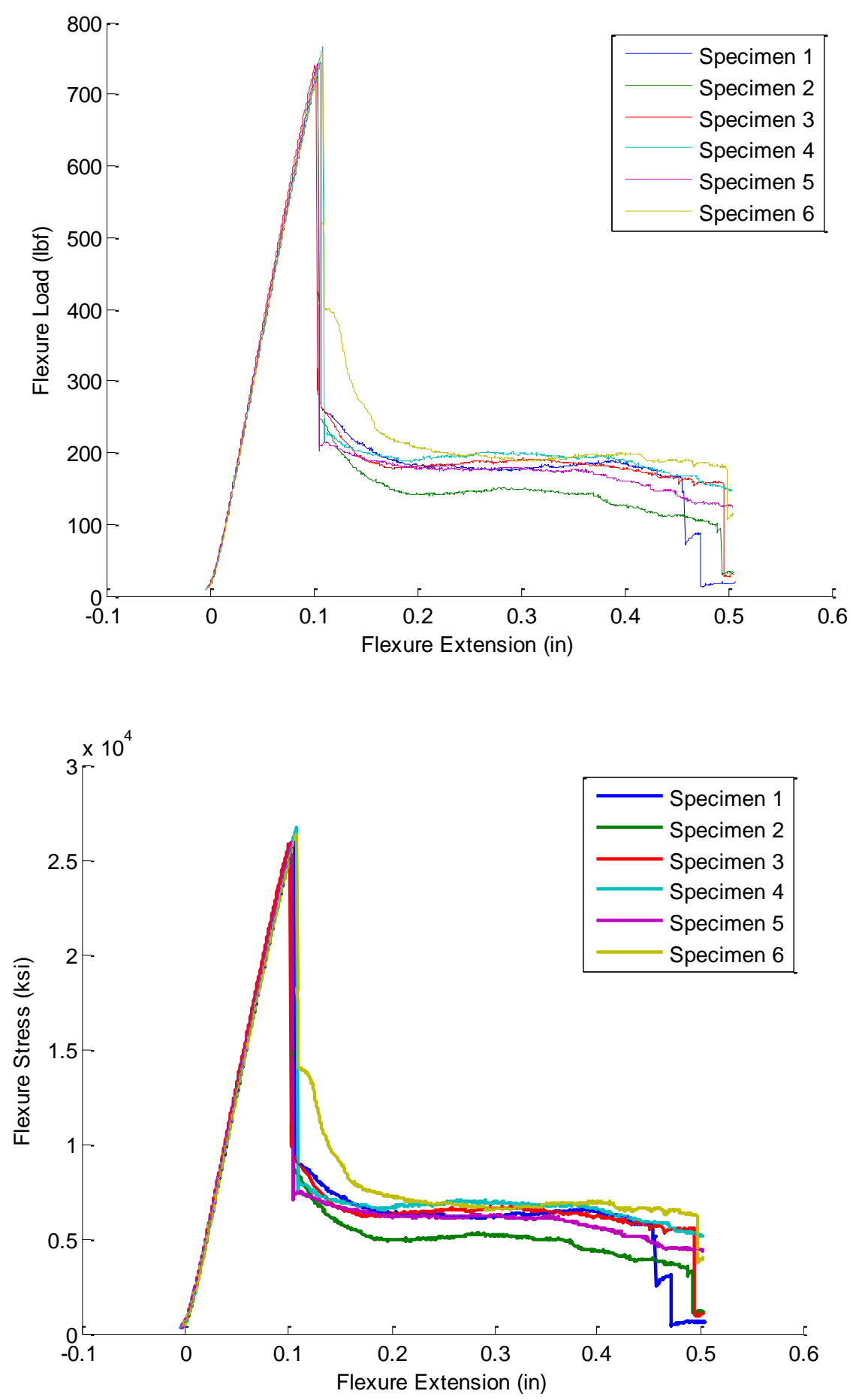
Figures 3.27.A. \& 3.28.A. 4/3 Configuration Load and Facing stress vs Extension Plots
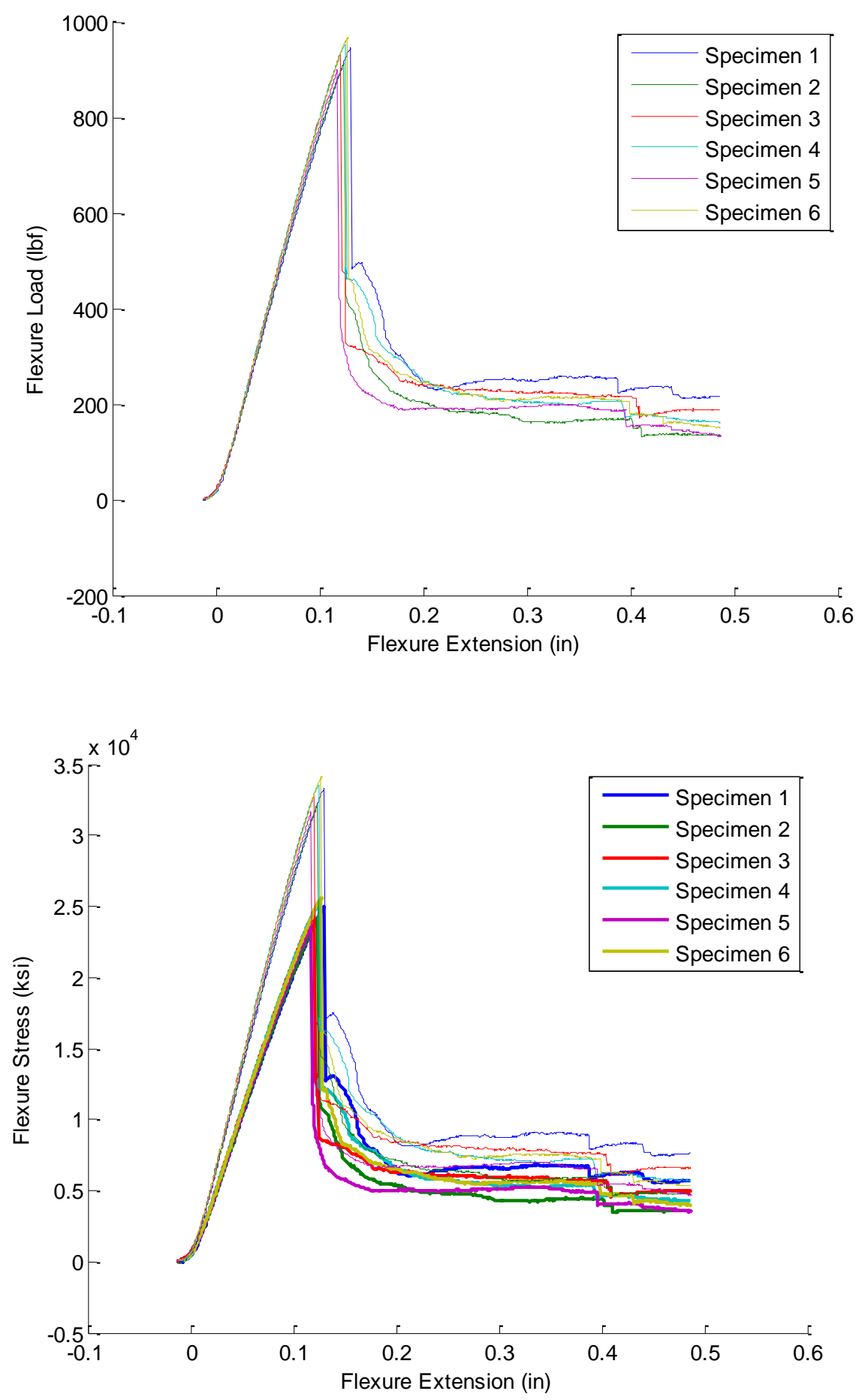
Figures 3.29.A. \& 3.30.A. 5/3 Configuration Load and Facing stress vs Extension Plots
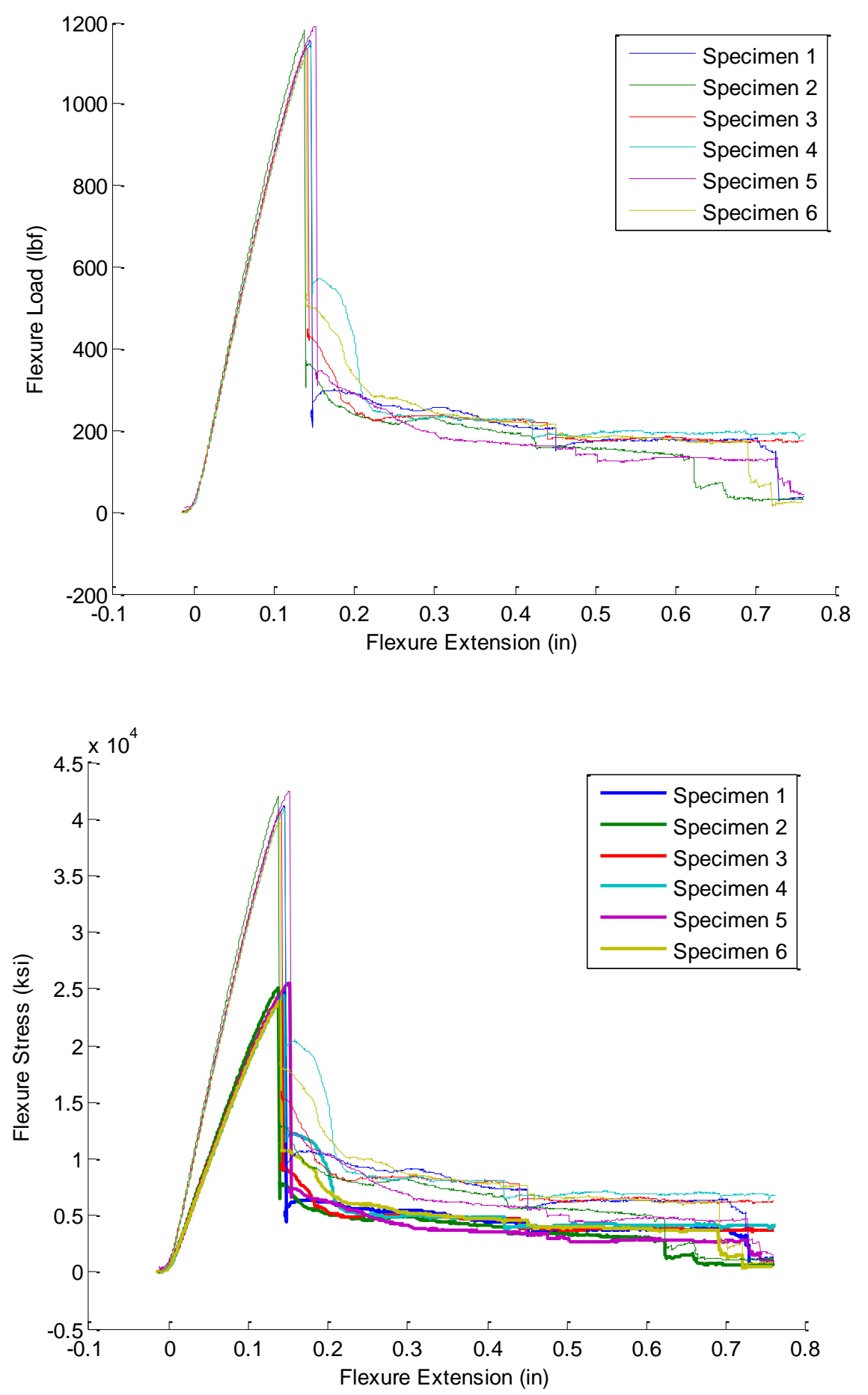
Figures 3.31.A. \& 3.32.A 1/4 Configuration Load and Facing stress vs Extension Plots
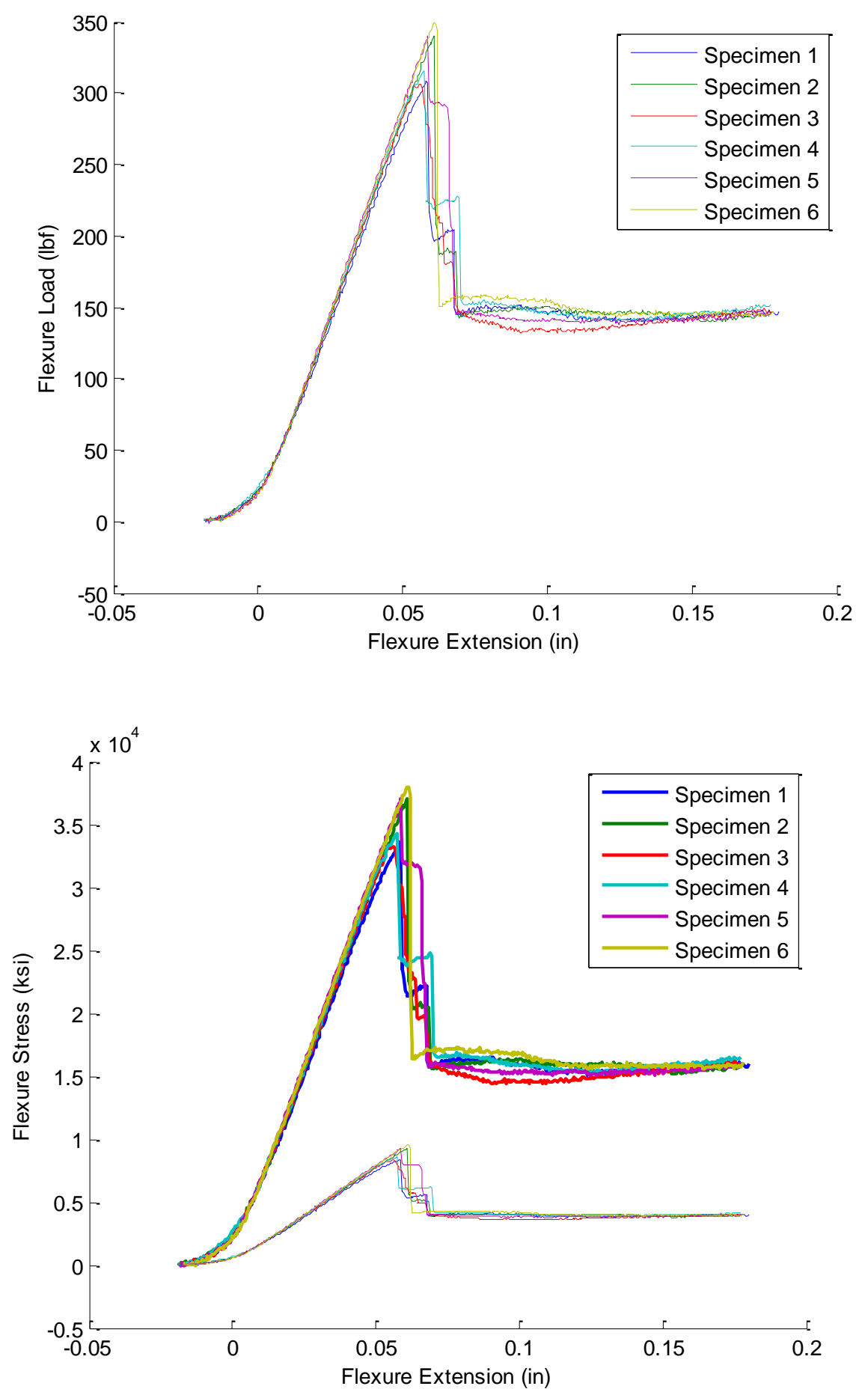
Figures 3.33.A. \& 3.34.A. 2/4 Configuration Load and Facing stress vs Extension Plots
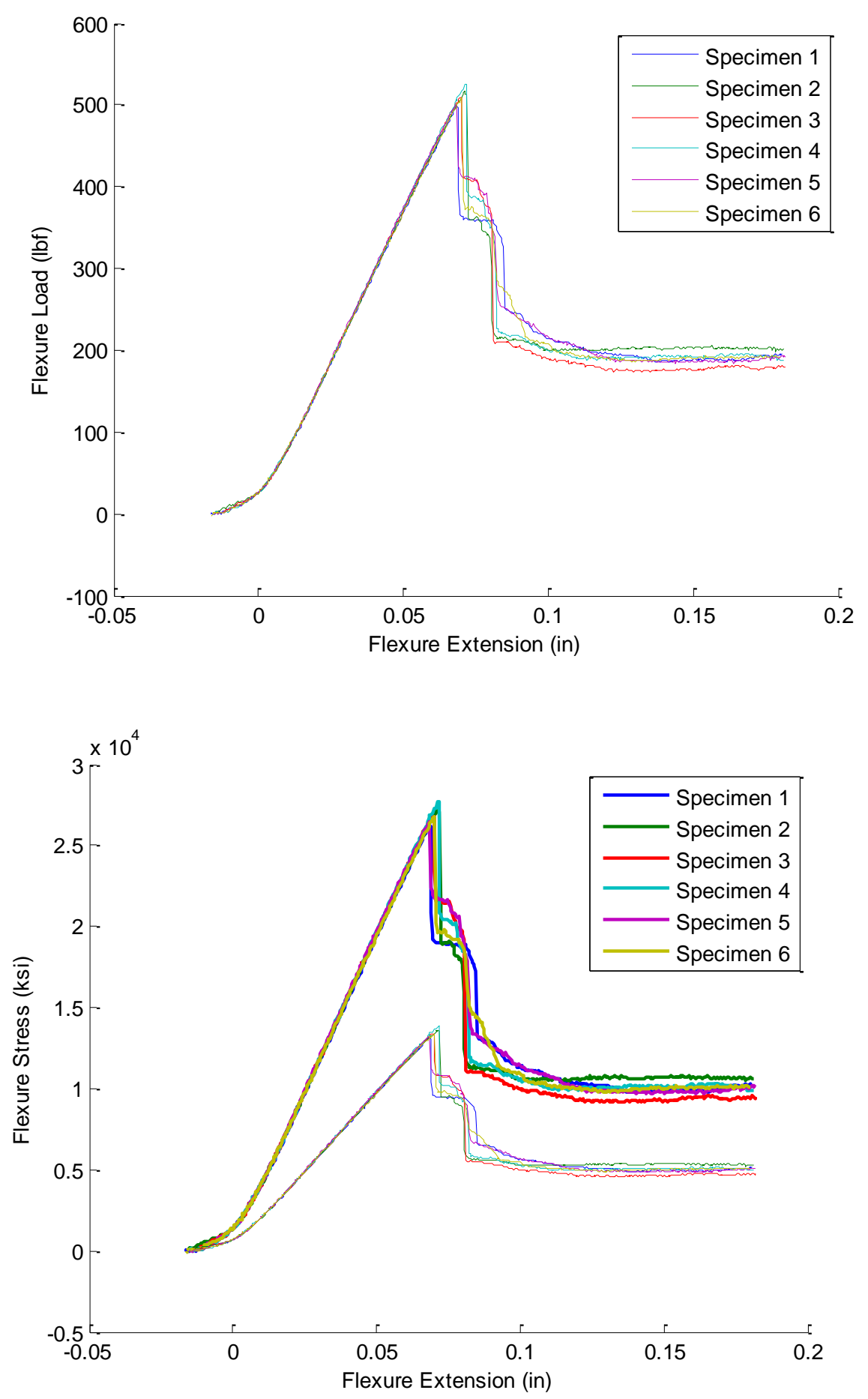
Figures 3.35.A. \& 3.36.A. 3/4 Configuration Load and Facing stress vs Extension Plots
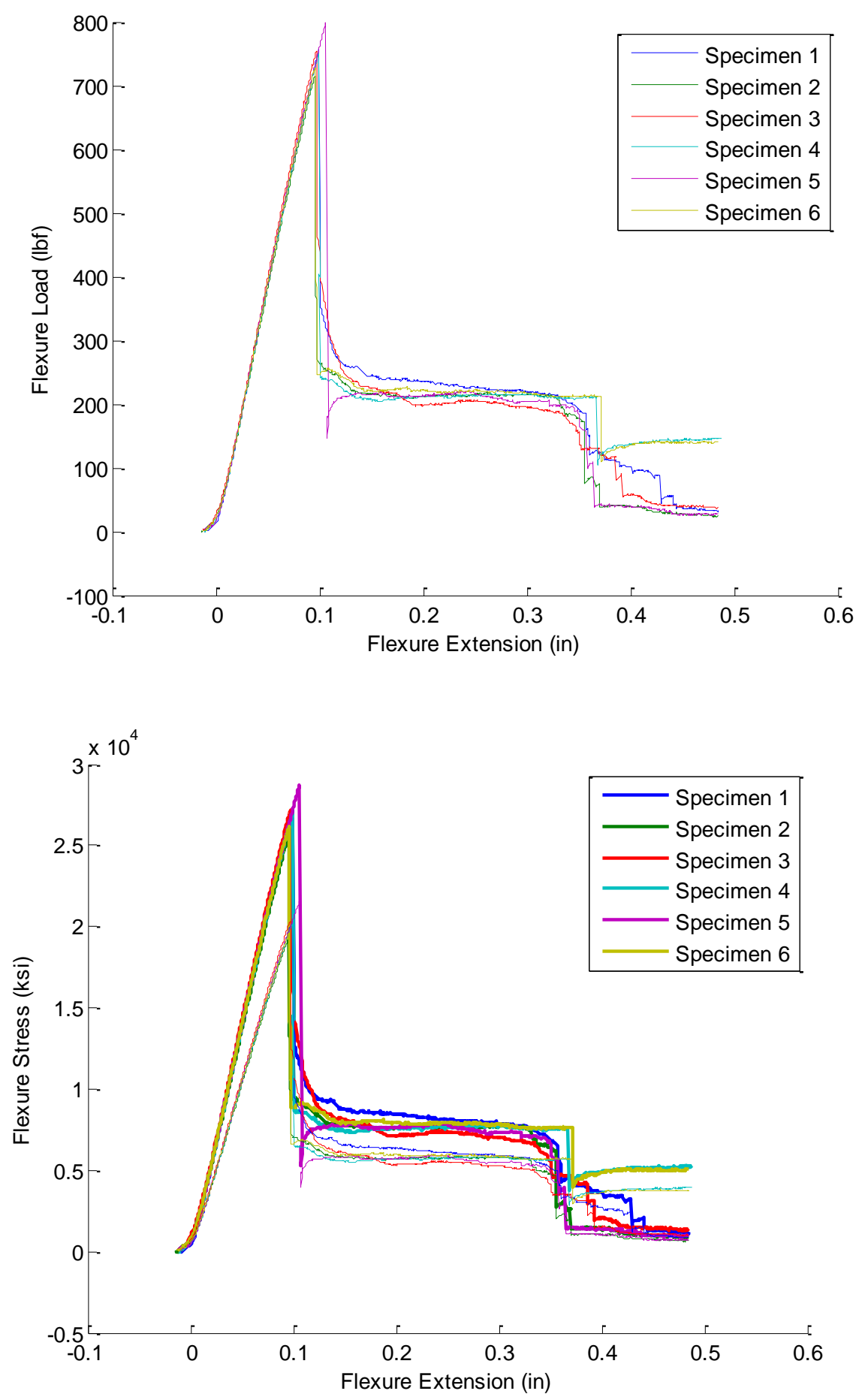
Figures 3.37.A. \& 3.38.A. 4/4 Configuration Load and Facing stress vs Extension Plots
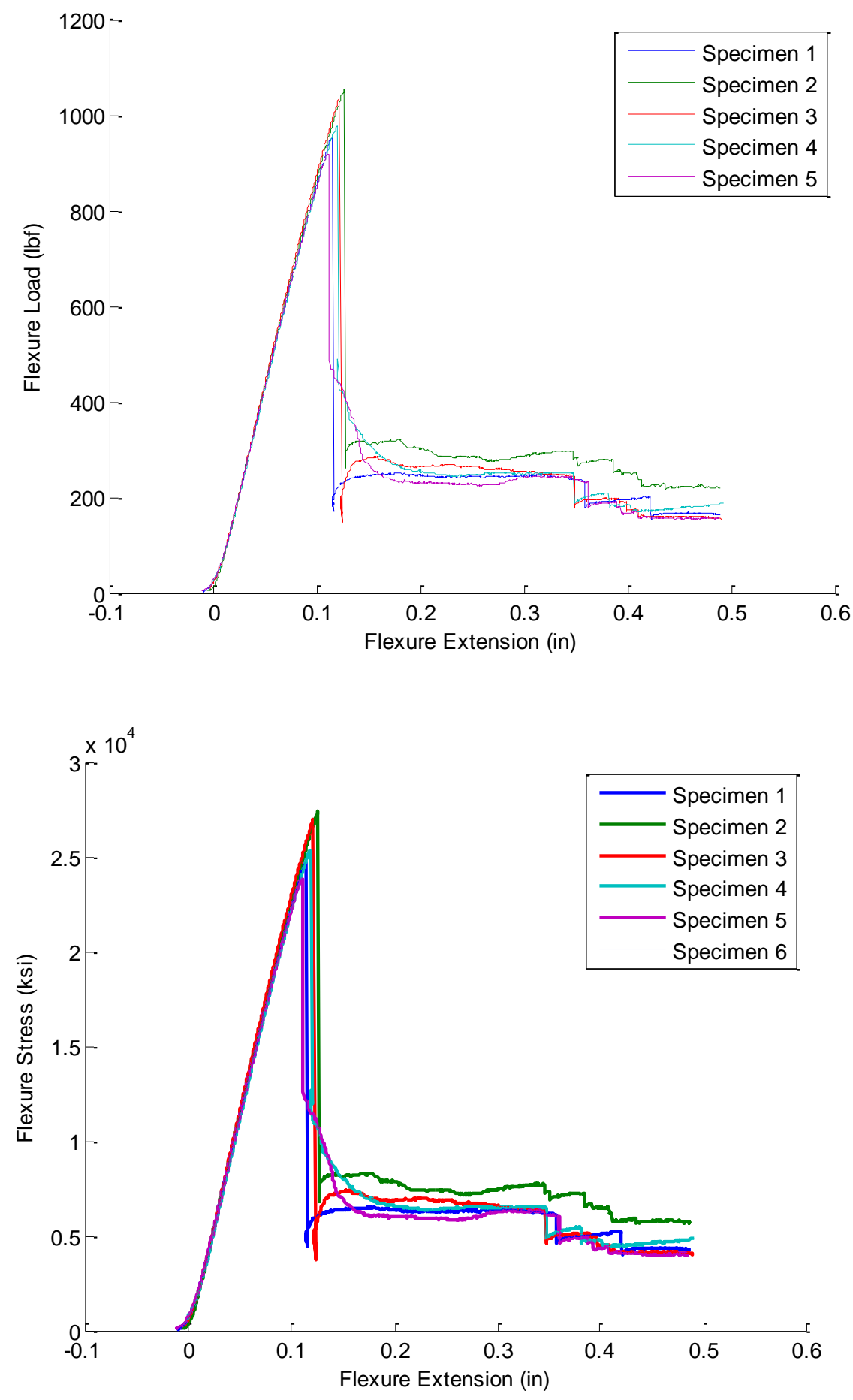
Figures 3.39.A. \& 3.40.A. 5/4 Configuration Load and Facing stress vs Extension Plots
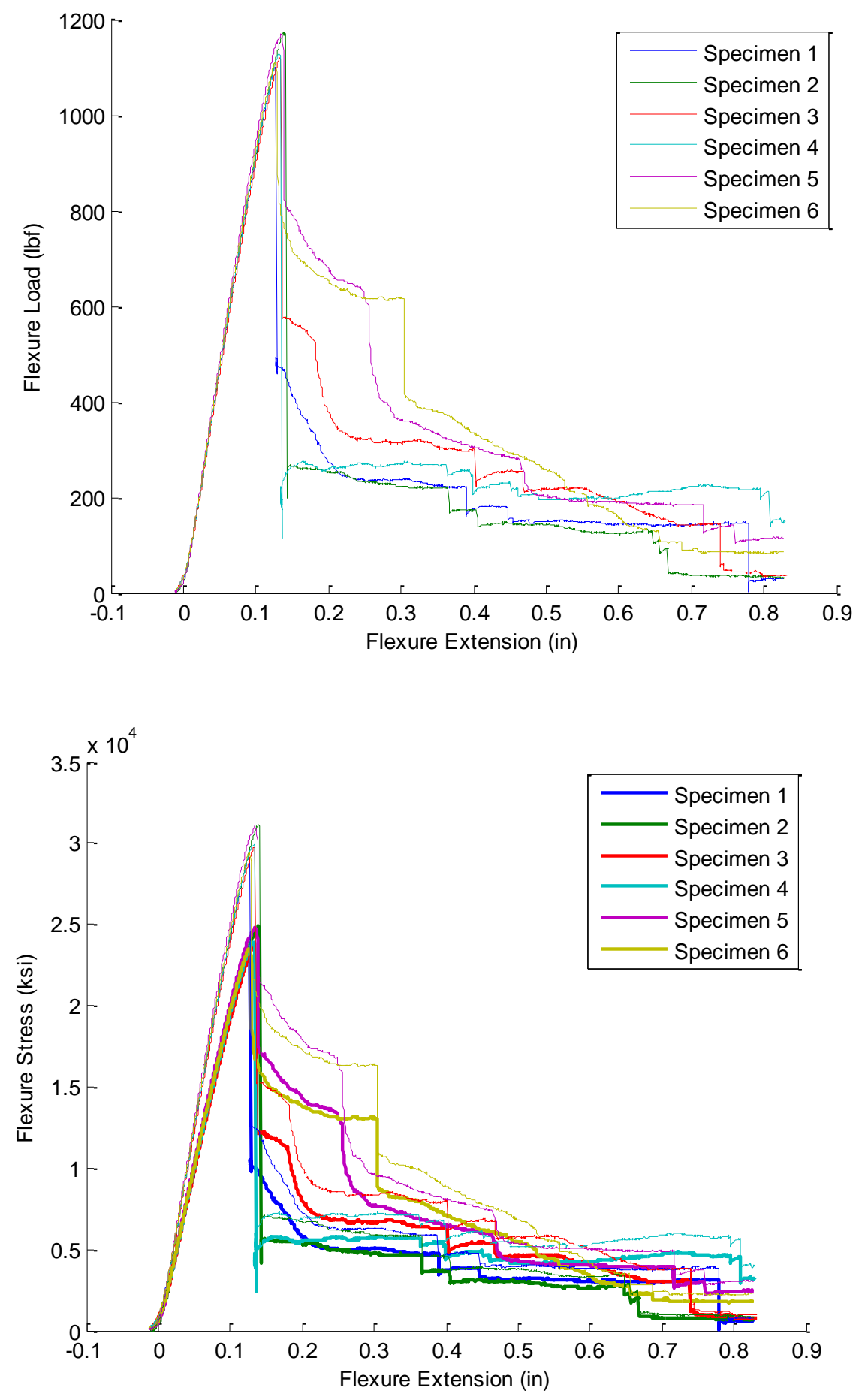
Figures 3.41.A. \& 3.42.A. 1/5 Configuration Load and Facing stress vs Extension Plots
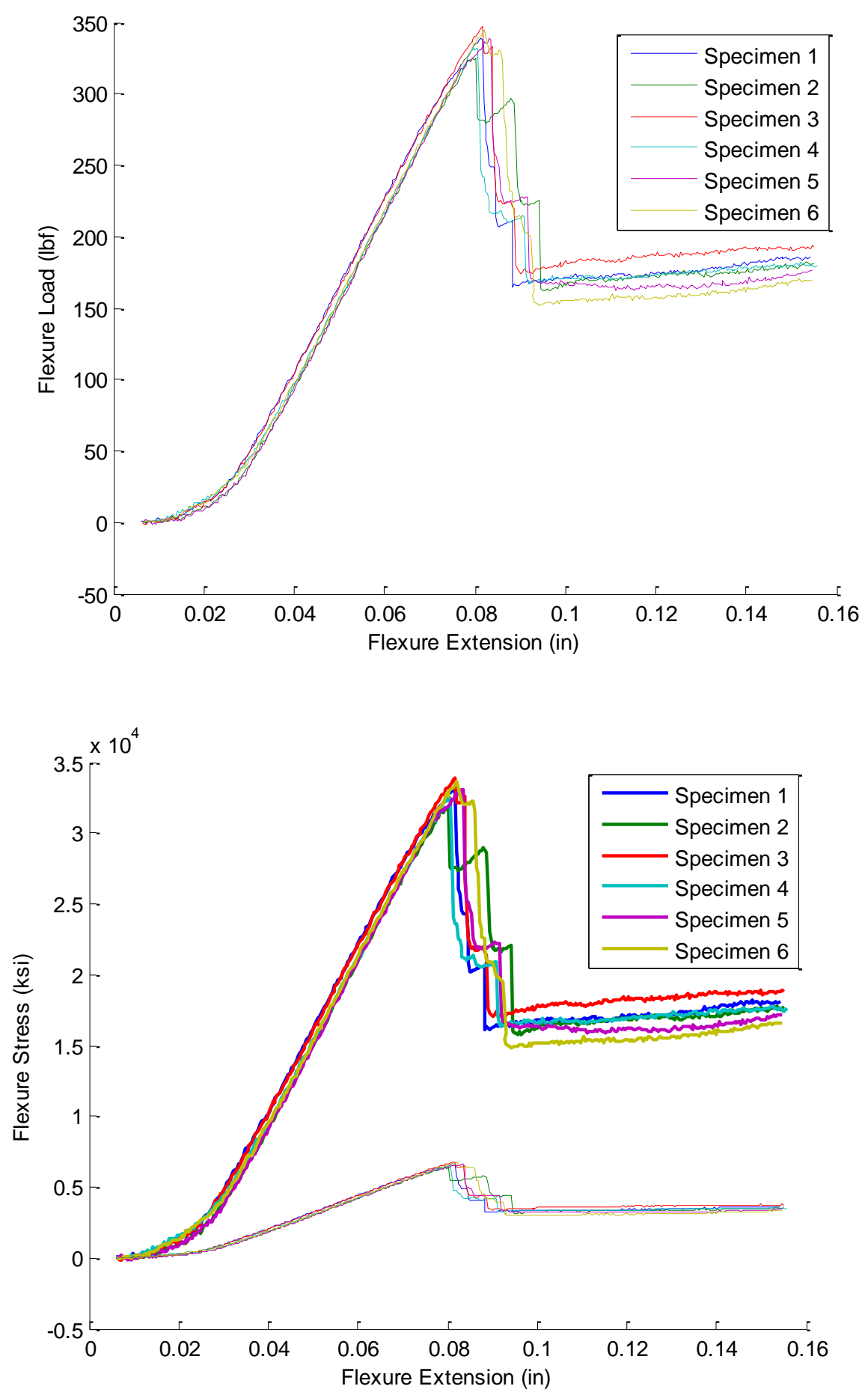
Figures 3.43.A. \& 3.44.A. 2/5 Configuration Load and Facing stress vs Extension Plots
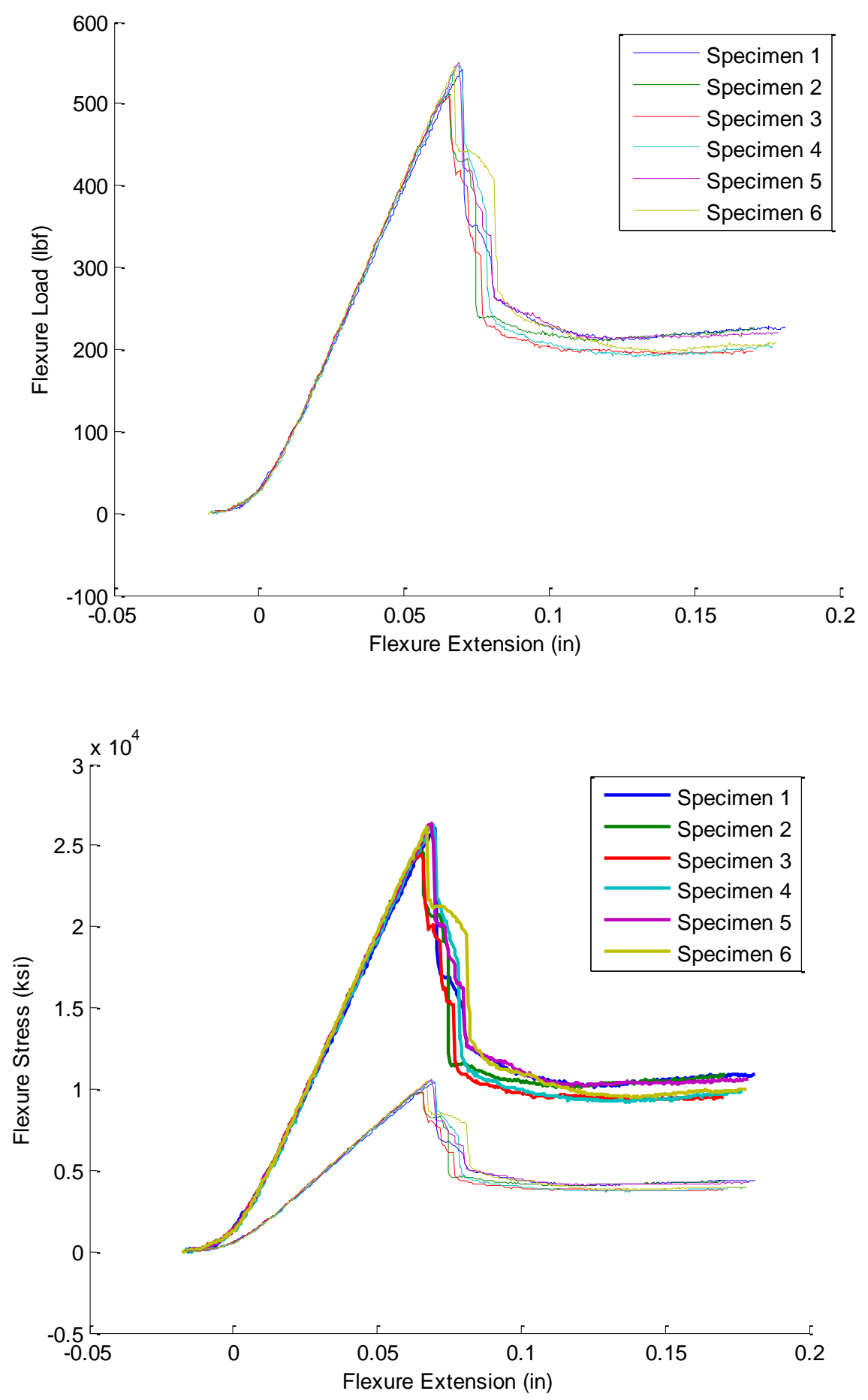
Figures 3.45.A. \& 3.46.A. 3/5 Configuration Load and Facing stress vs Extension Plots
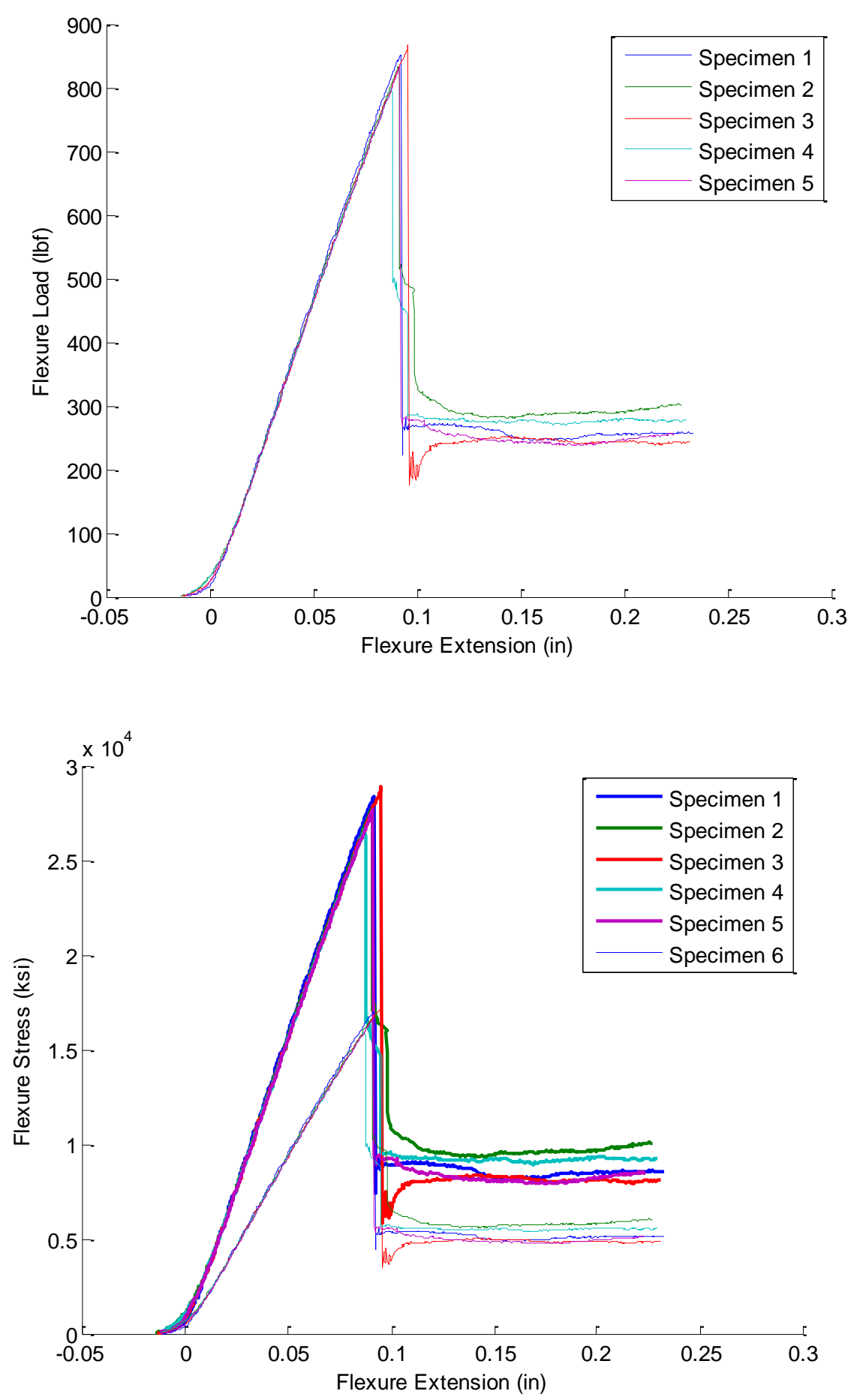
Figures 3.47.A. \& 3.48.A. 4/5 Configuration Load and Facing stress vs Extension Plots
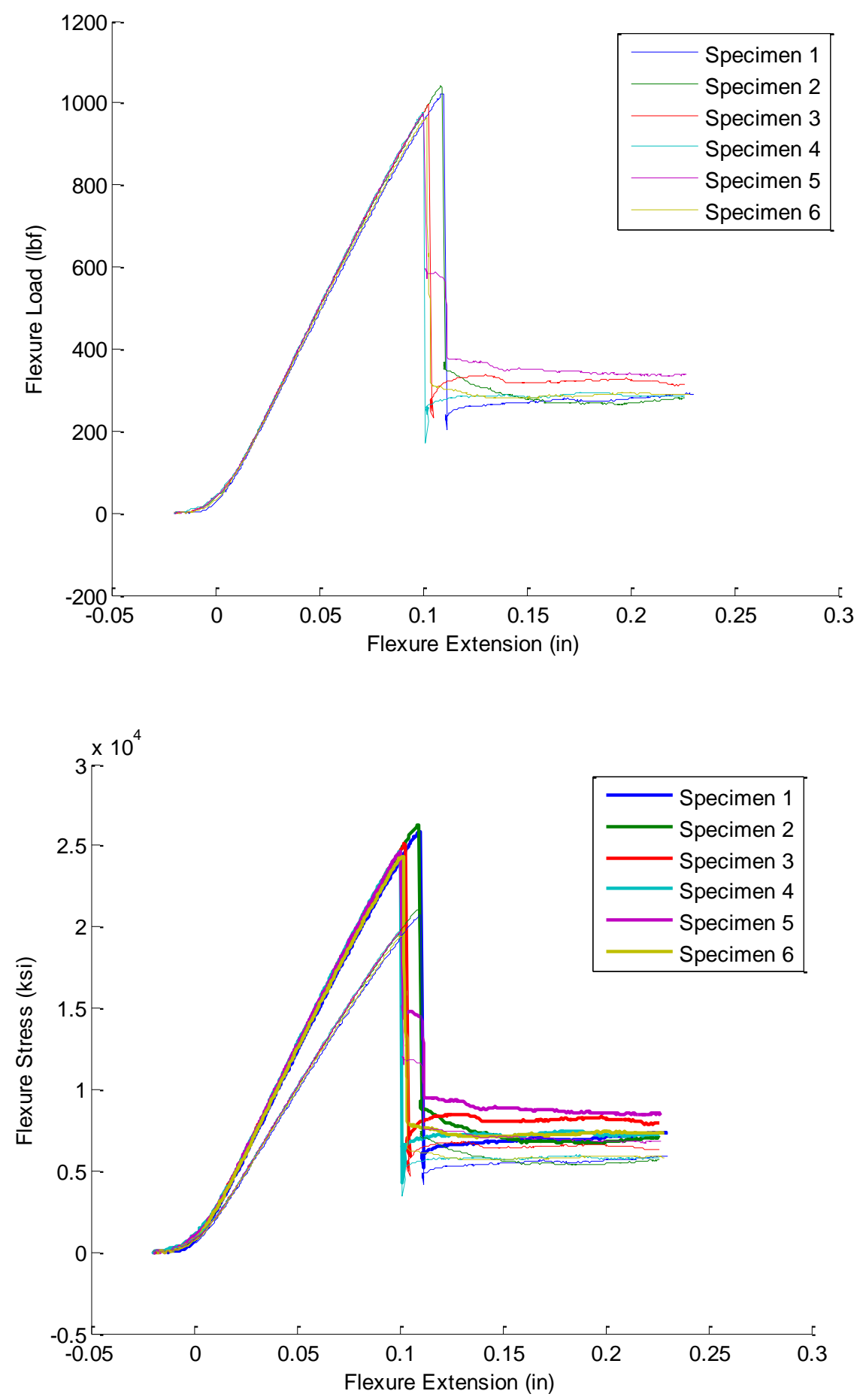
Figures 3.49.A. \& 3.50.A. 5/5 Configuration Load and Facing stress vs Extension Plots
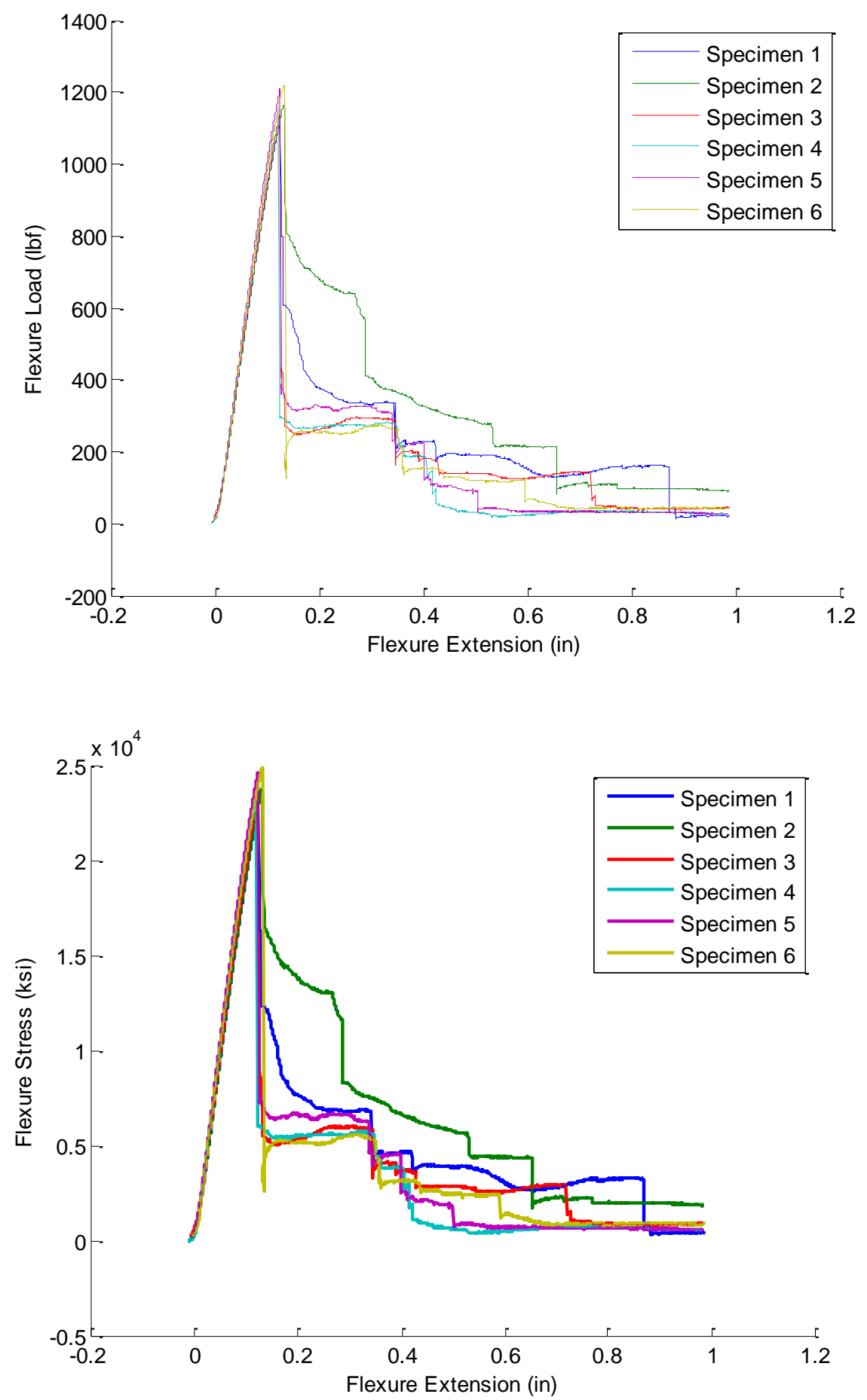


\section{A.4 Finite Element Analysis - Core Ribbon Direction}

Figures 4.1.A. \& 4.2.A 15 Degree Core FEA Stress and Deflection Plots
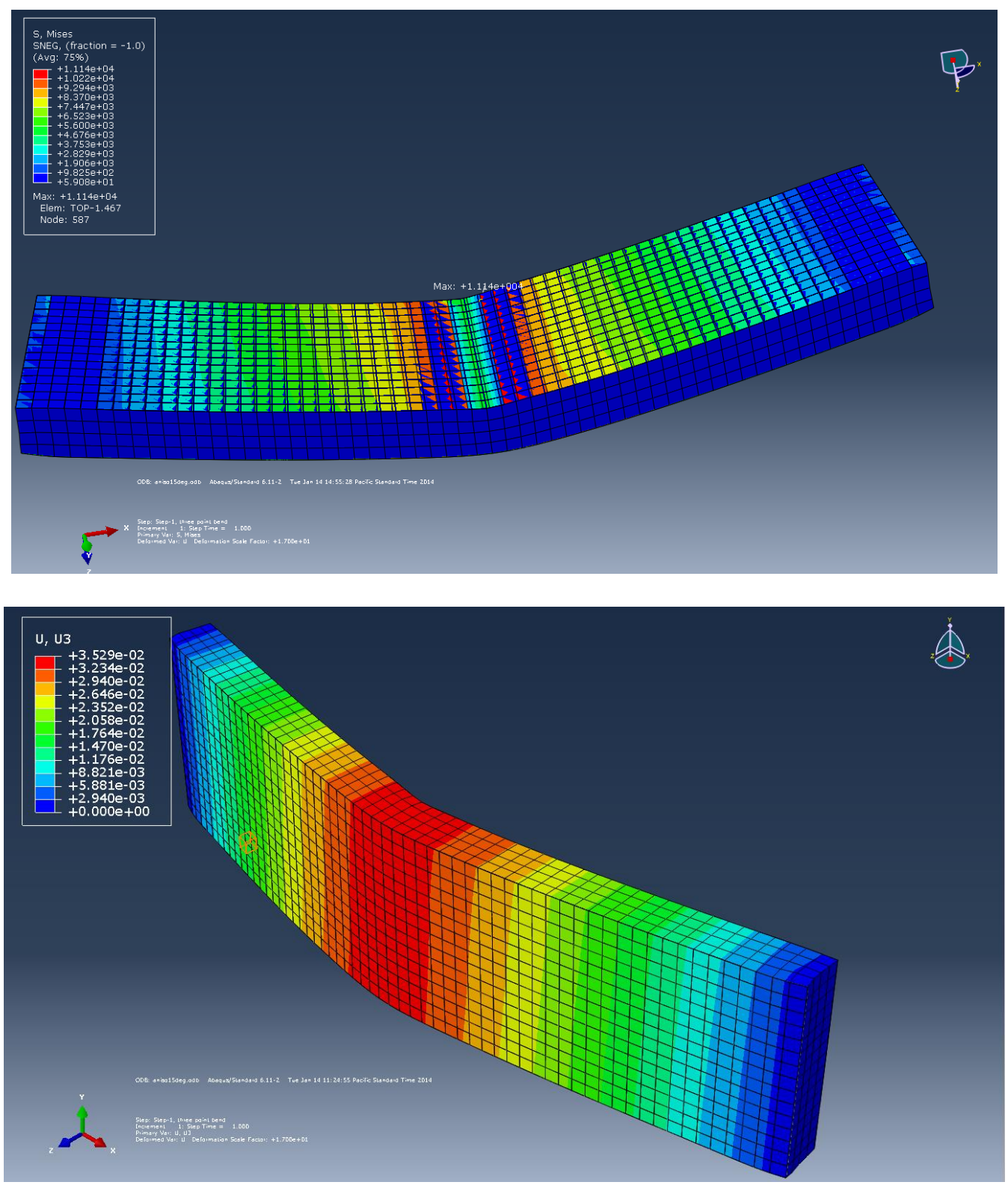
Figures 4.3.A. \& 4.4.A 30 Degree Core FEA Stress and Displacement plots
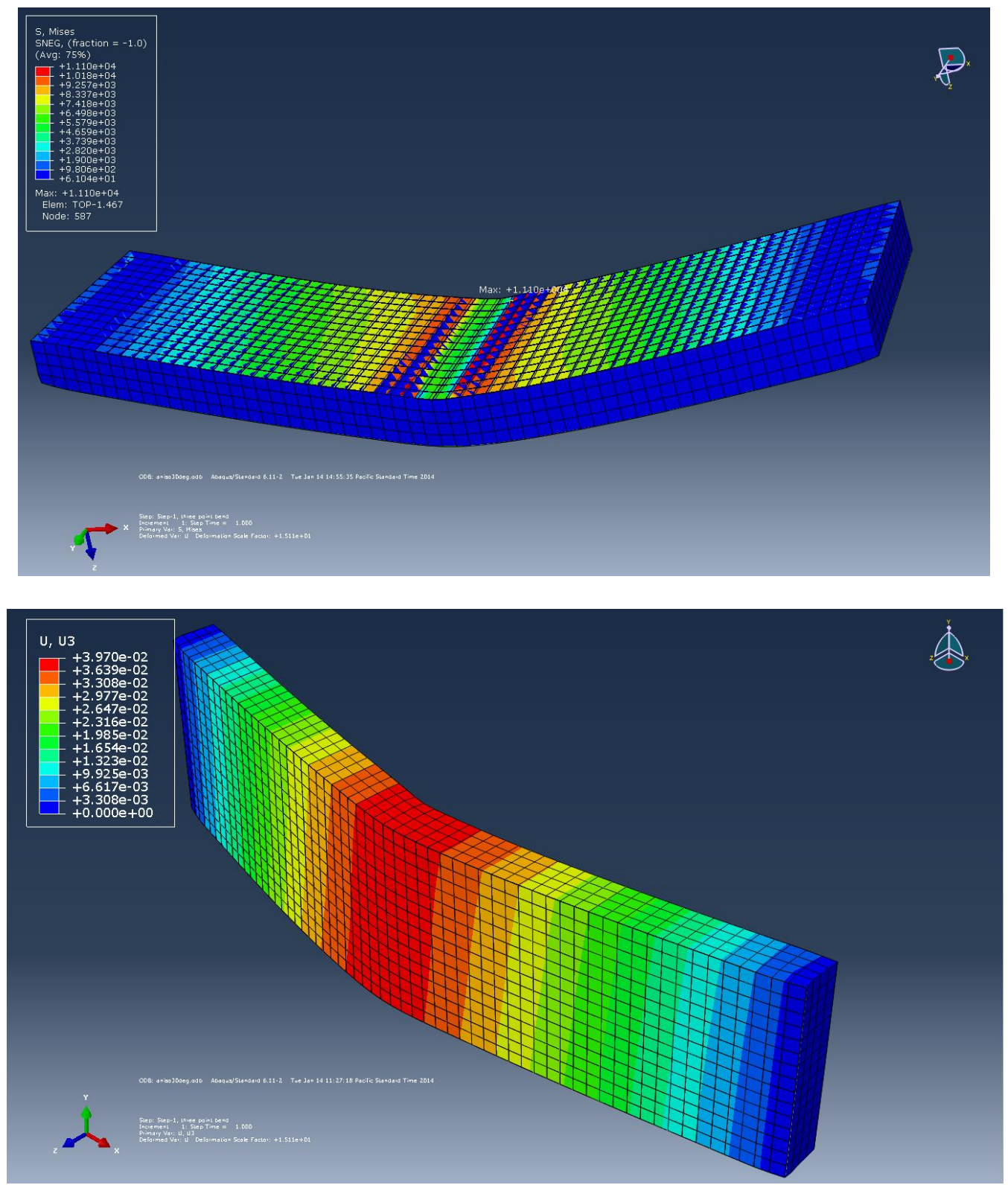
Figures 4.5.A. \& 4.6.A 45 Degree Core FEA Stress and Displacement Plots
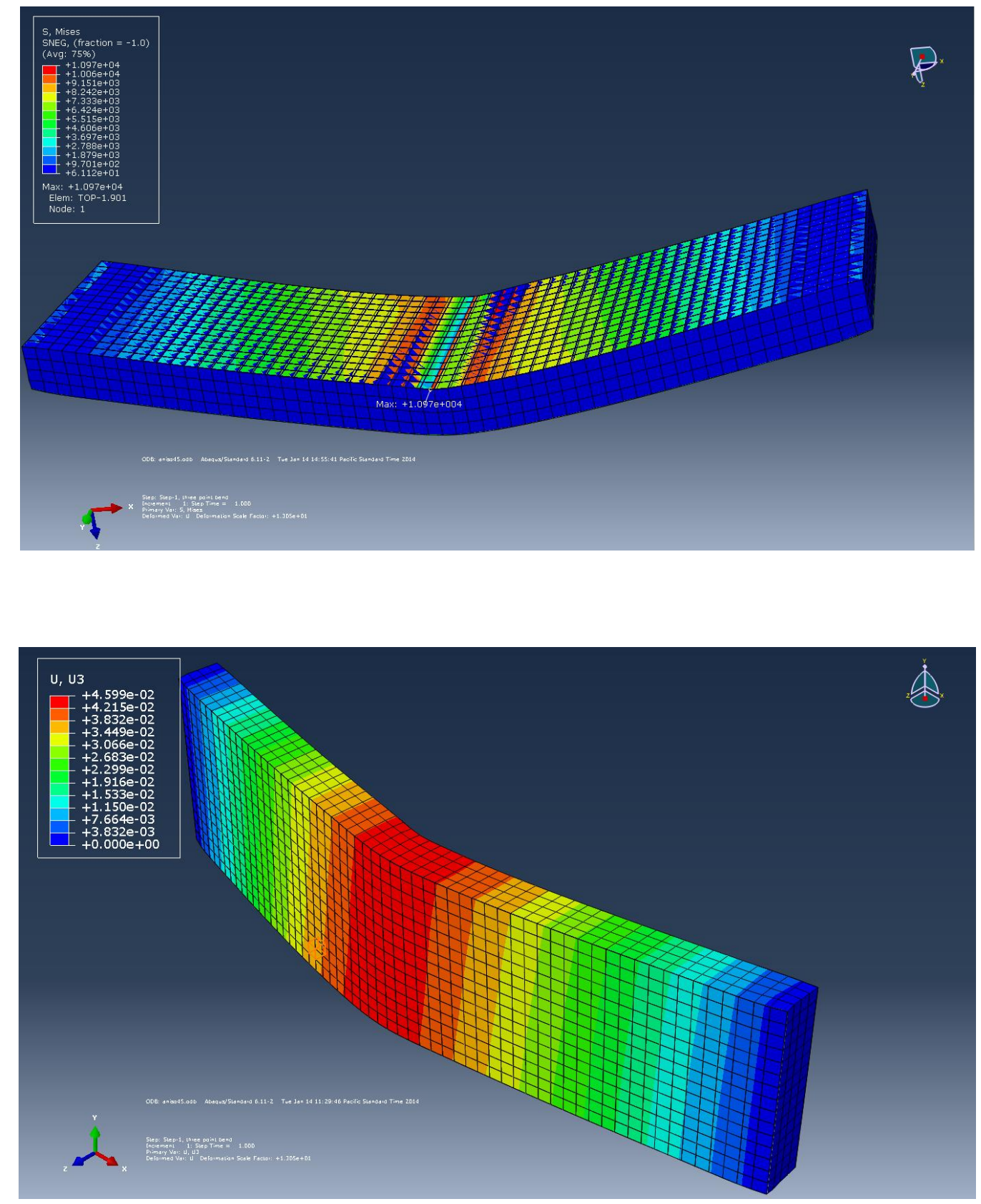
Figures 4.7.A. \& 4.8.A 60 Degree Core FEA Stress and Displacement Plots
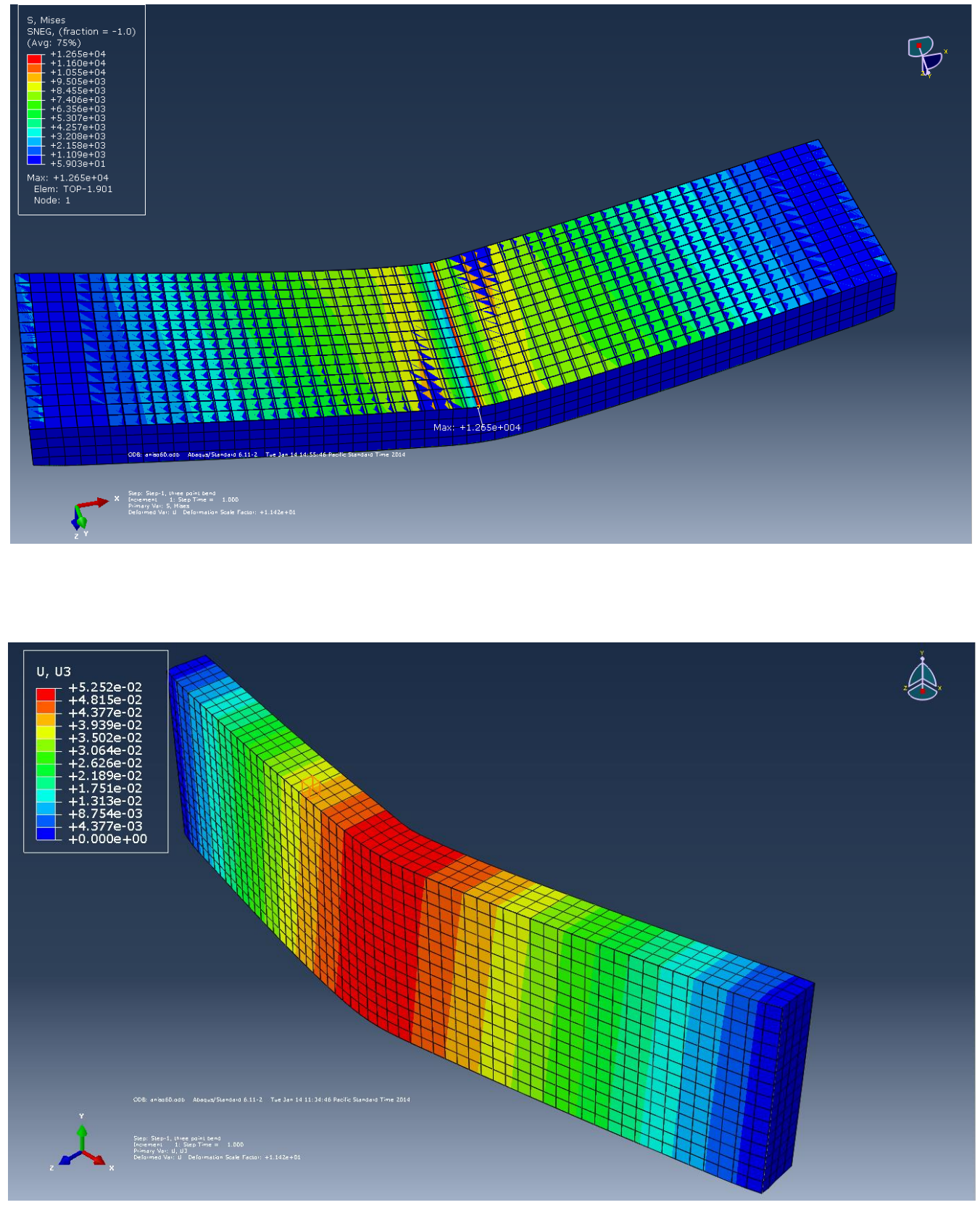
Figures 4.9.A. \& 4.10.A 75 Degree Core FEA Stress and Displacement Plots
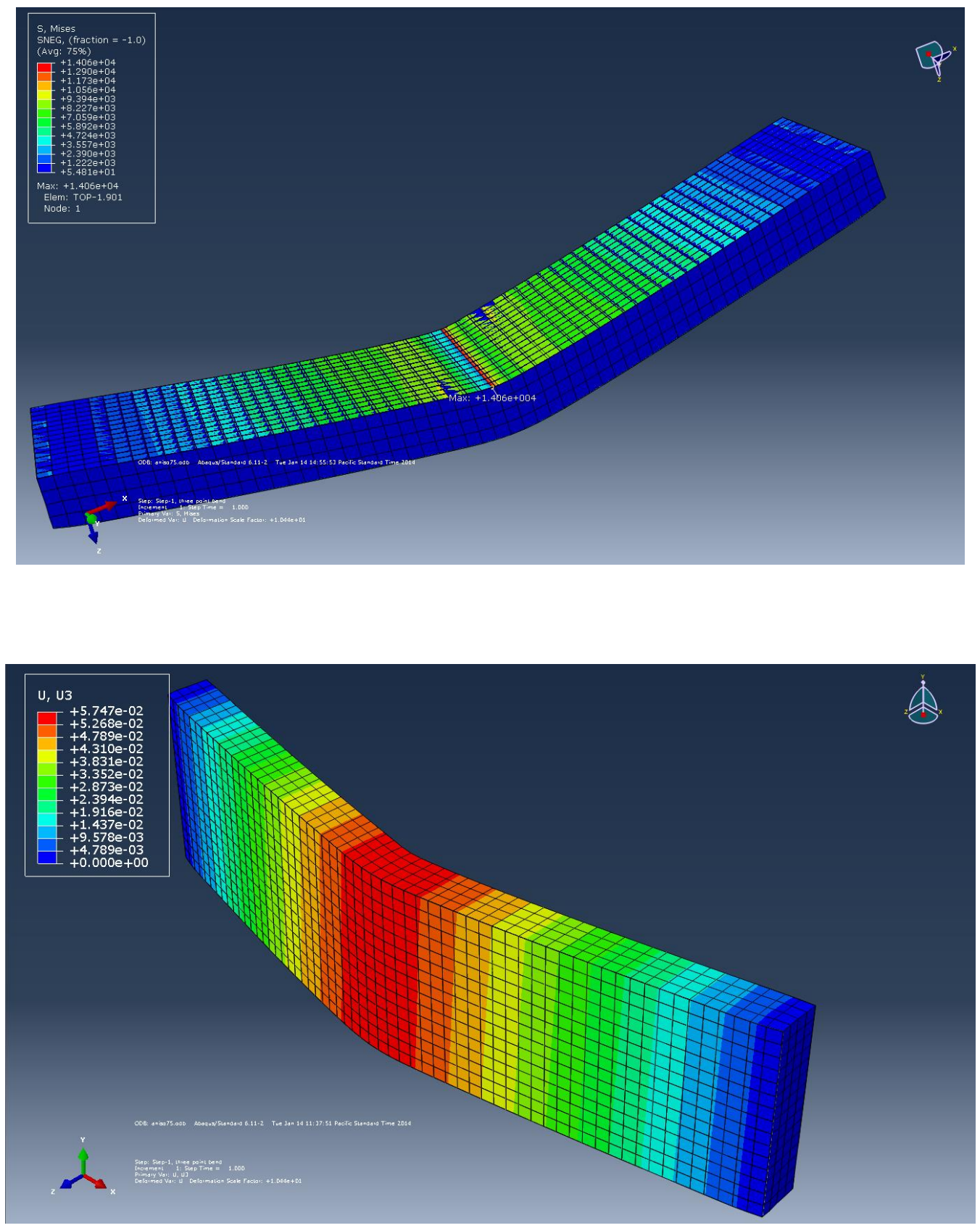
Figures 4.11.A. \& 4.12.A 90 Degree Core FEA Stress and Displacement Plots
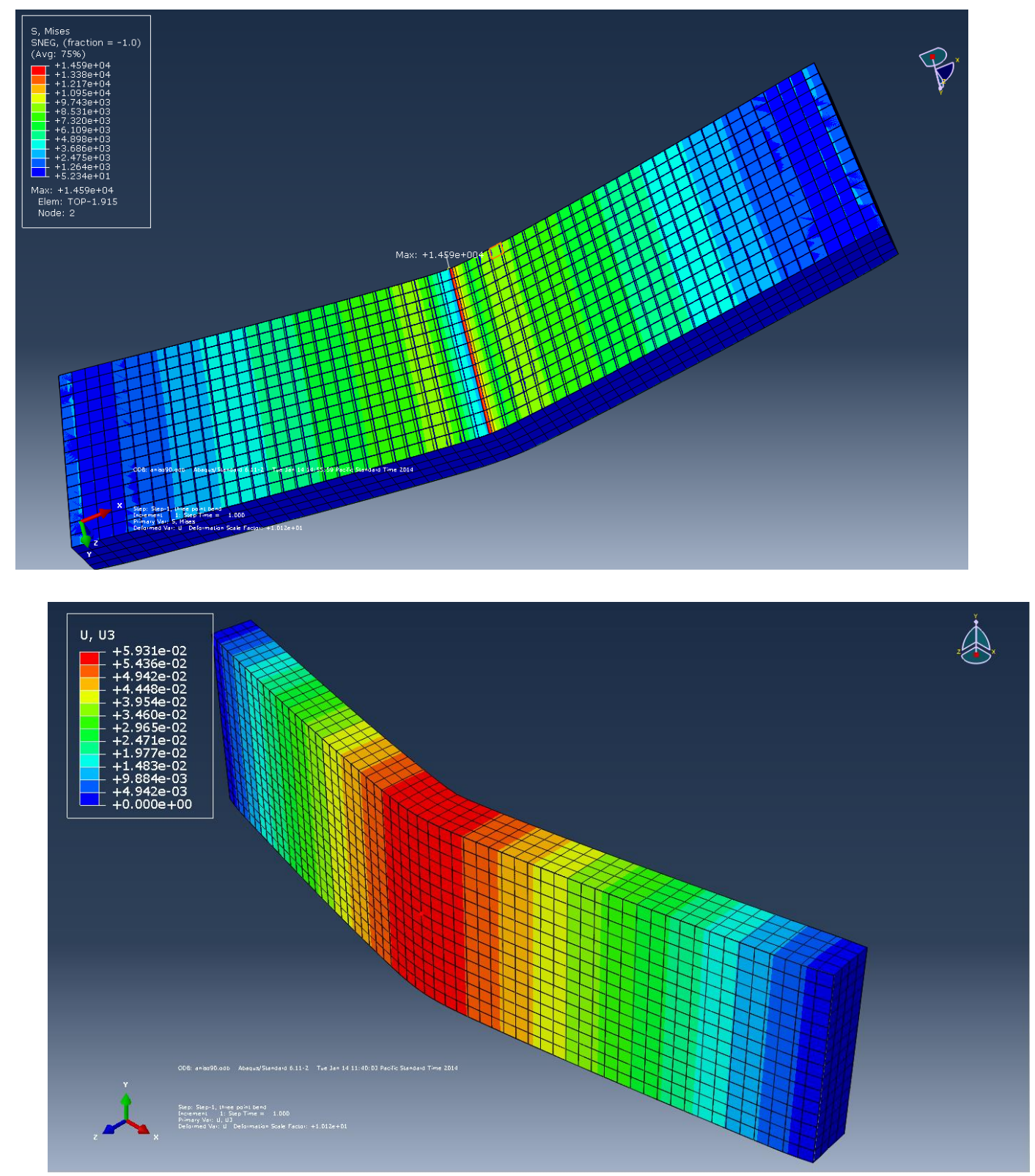


\section{A.5 Core Ribbon Direction Failure Mode Maps}

\section{Figure 5.1.A 15 Degree Core Failure Mode Map}

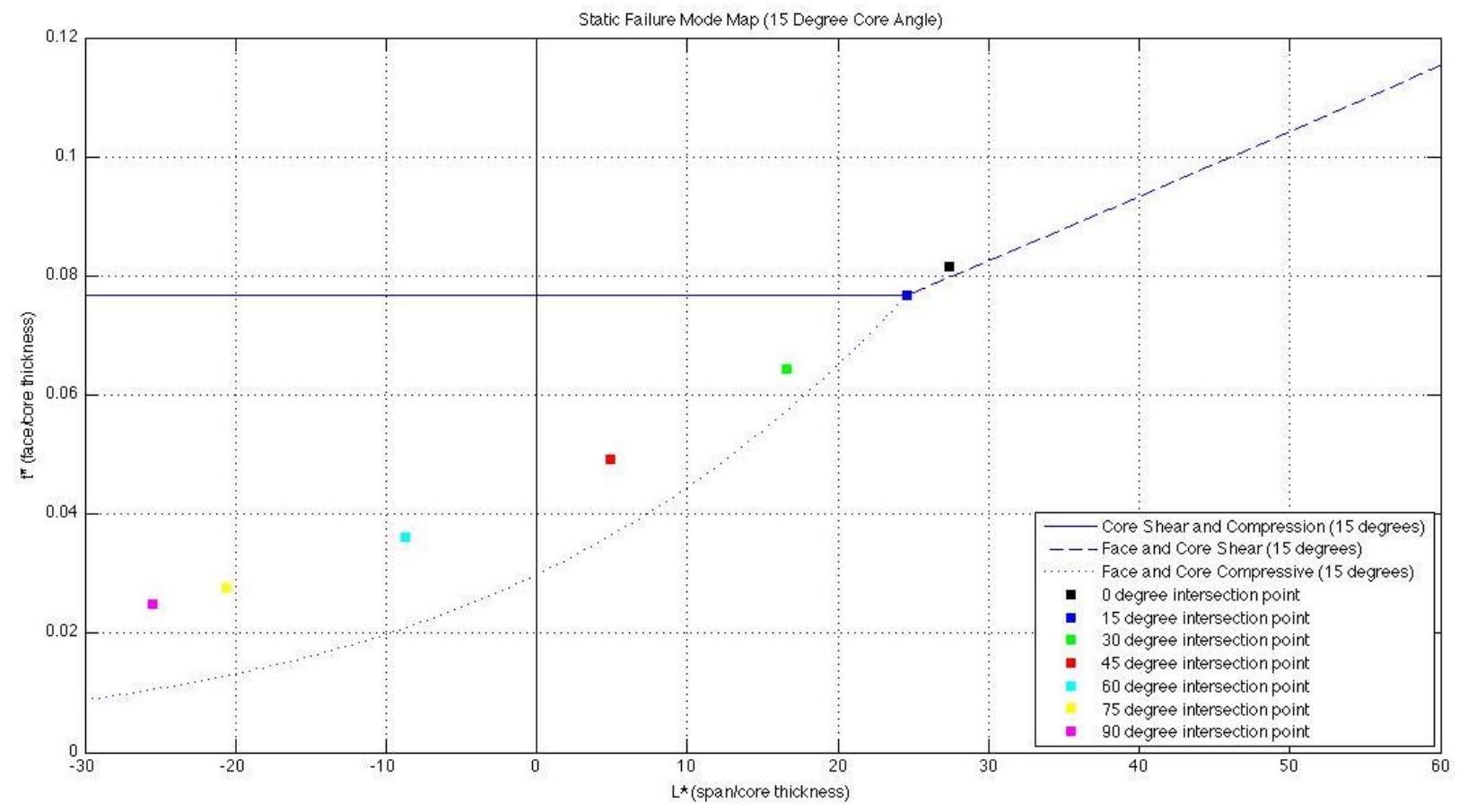


Figure 5.2.A 30 Degree Core Failure Mode Map

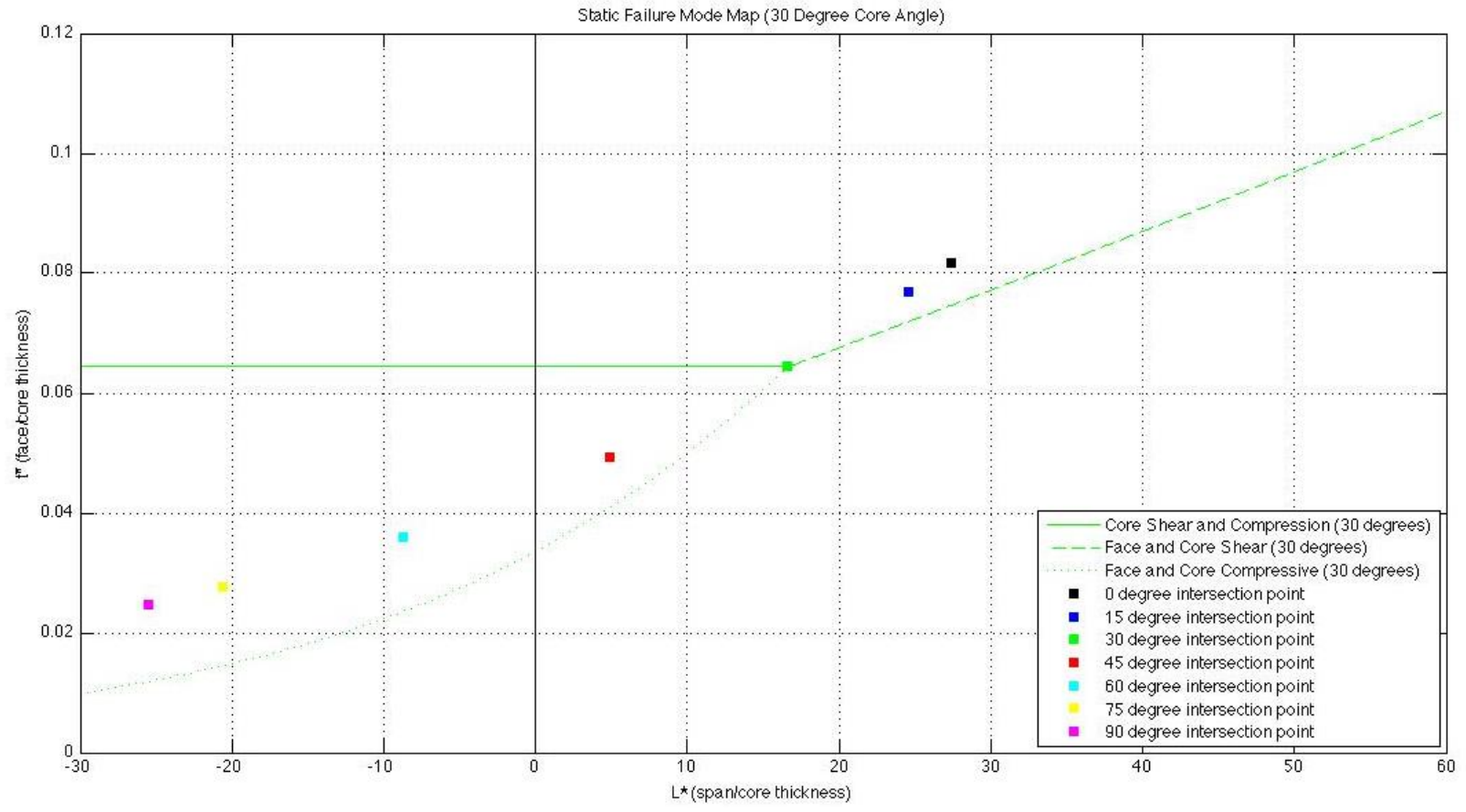

Figure 5.3.A 45 Degree Core Failure Mode Map 


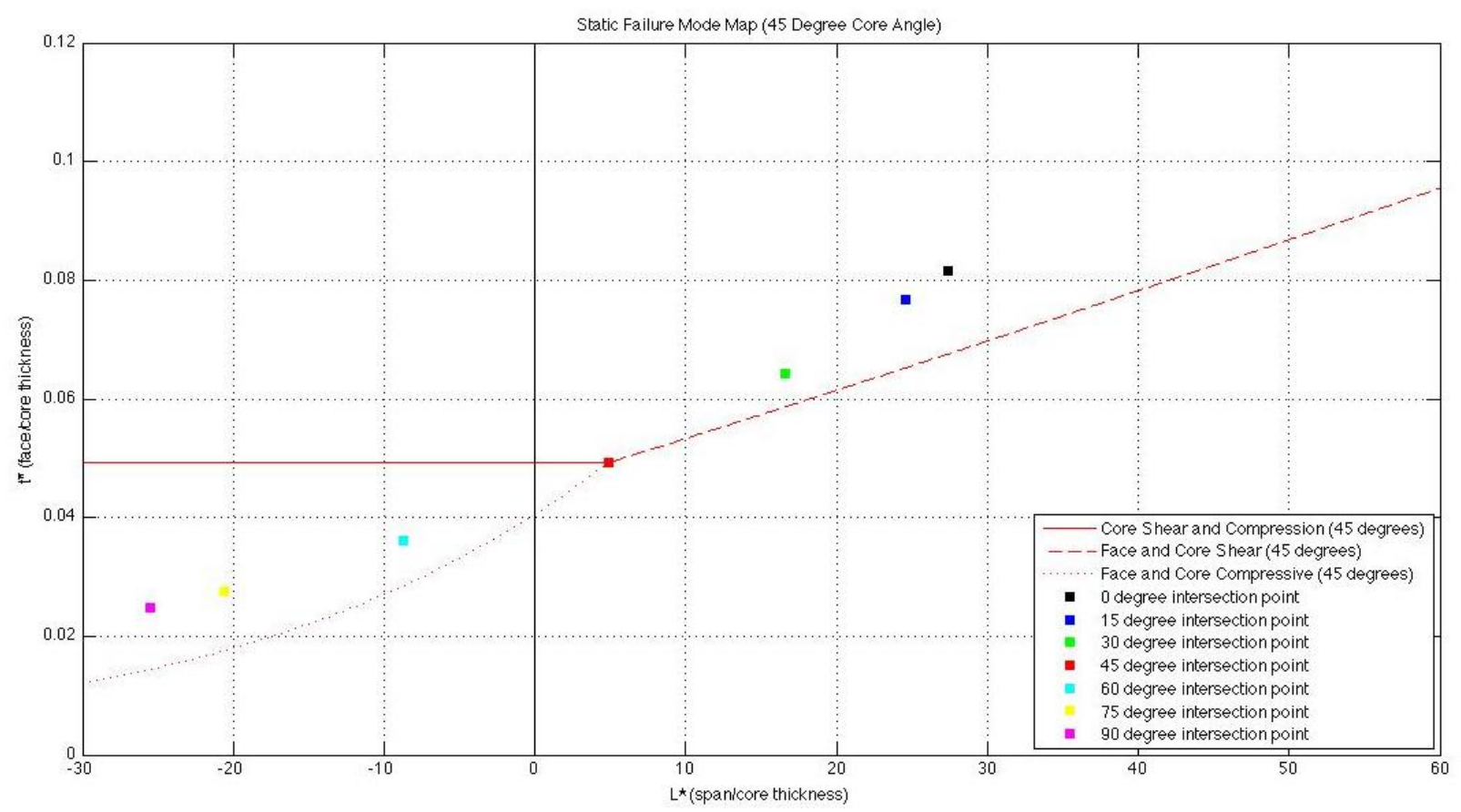

Figure 5.4.A 60 Degree Core Failure Mode Map

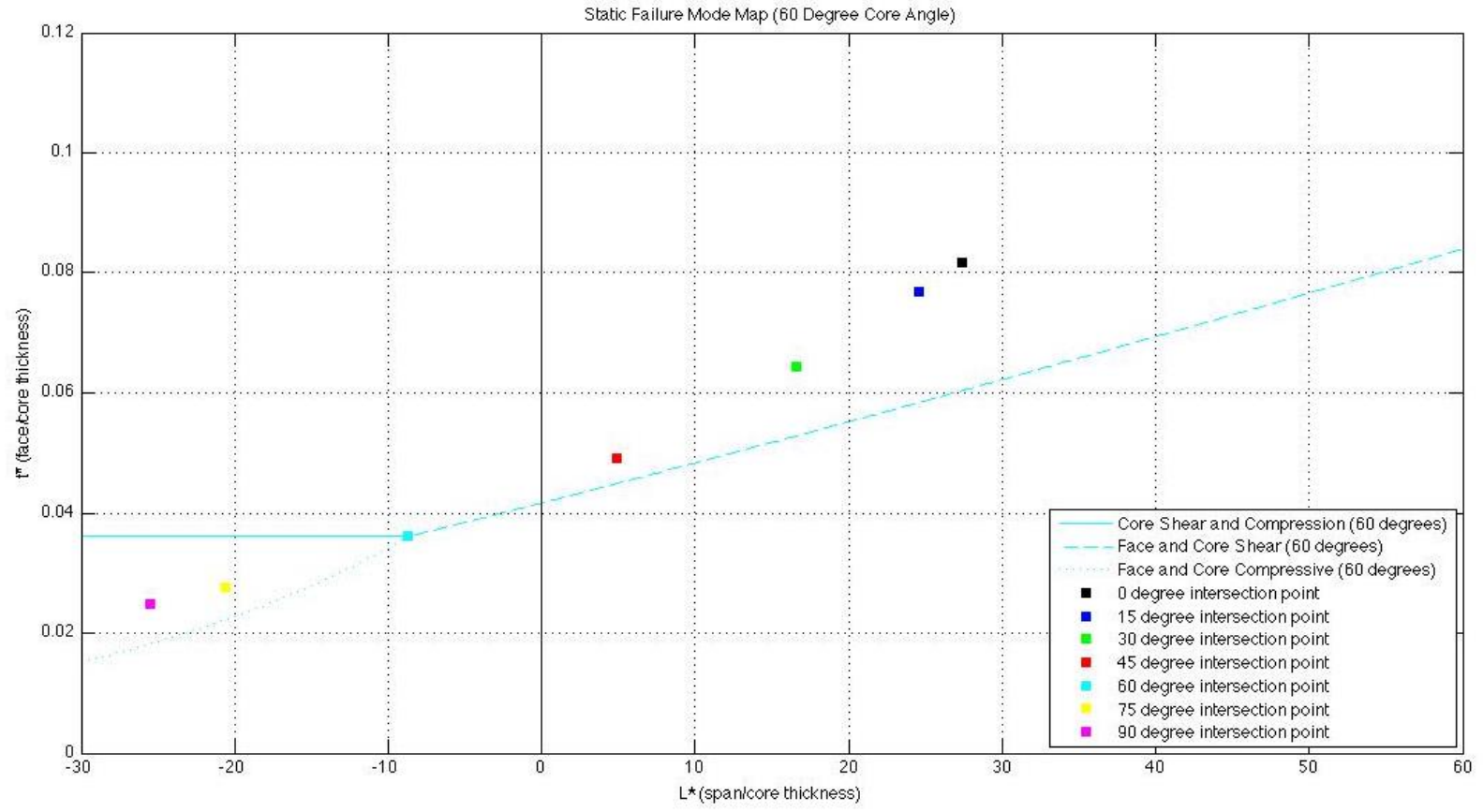

Figure 5.5.A 75 Degree Core Failure Mode Map 


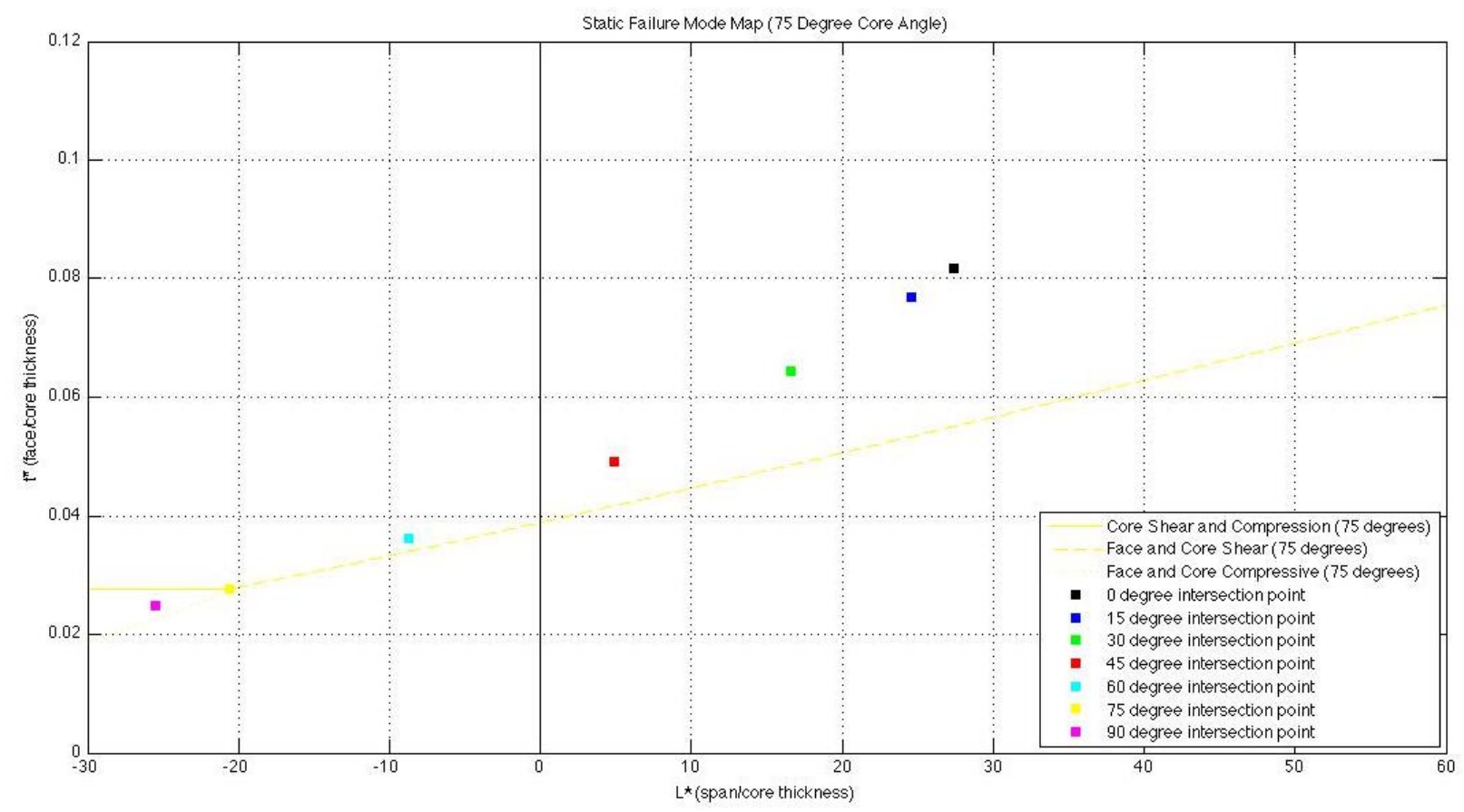

Figure 5.6.A 90 Degree Core Failure Mode Map

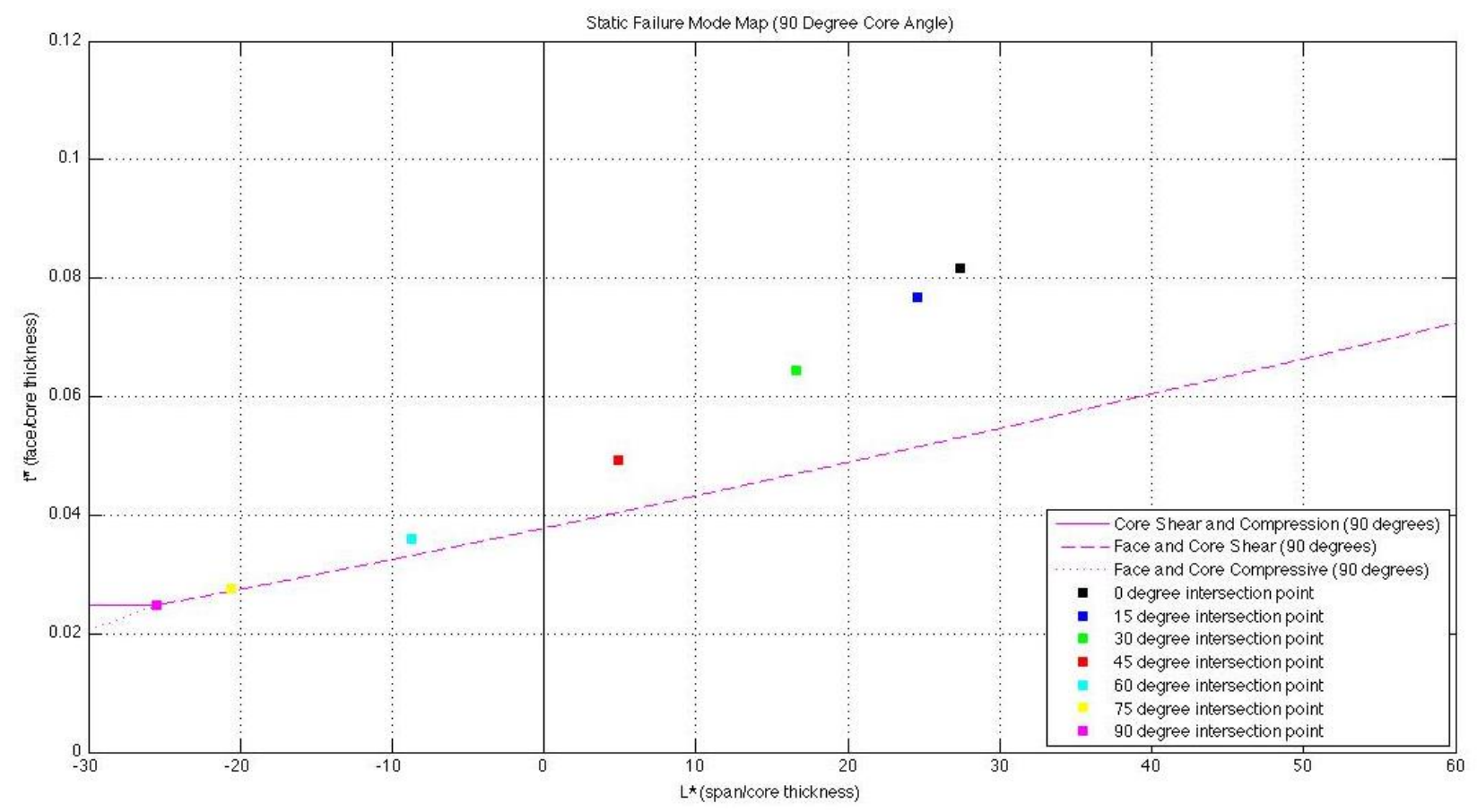




\section{A.6 Varying Face Sheet thicknesses Failure Loads}

Figure 6.1.A Varying Top Face Sheet thicknesses Failure Loads for 2 Bottom Layers

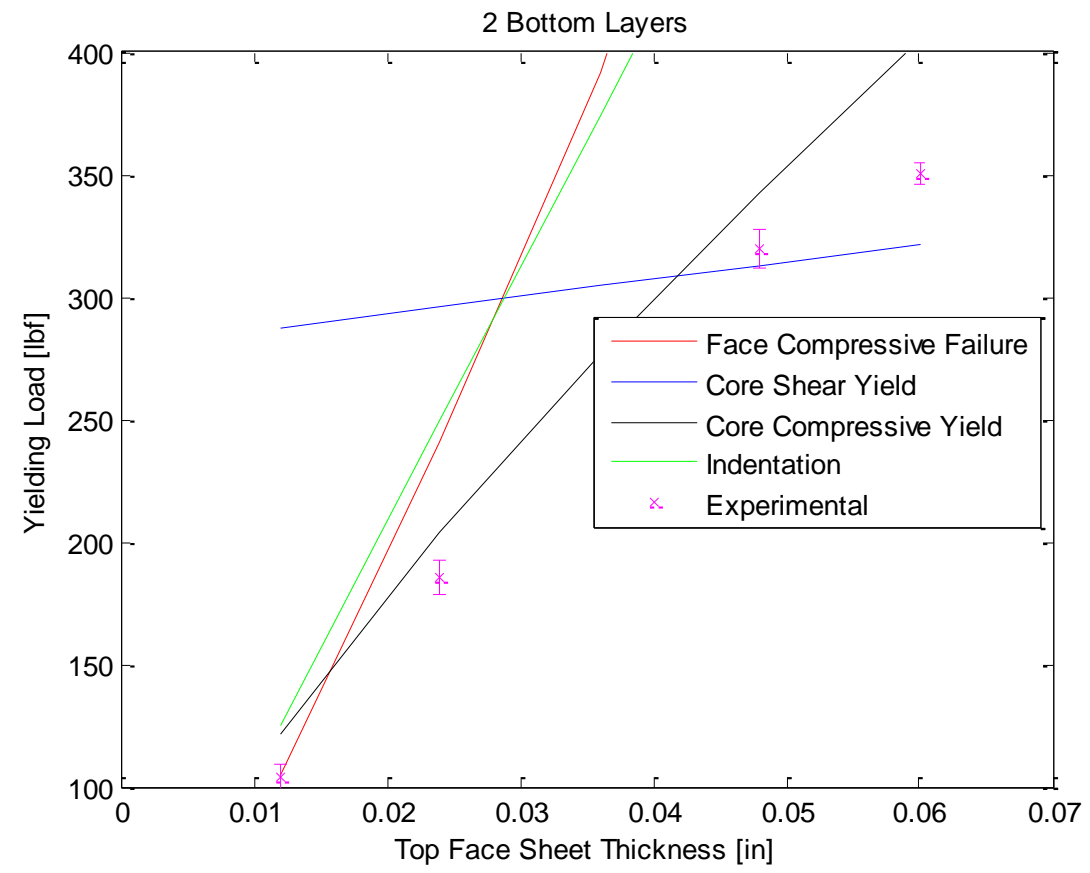

Figure 6.2.A Varying Top Face Sheet thicknesses Failure Loads for 3 Bottom Layers

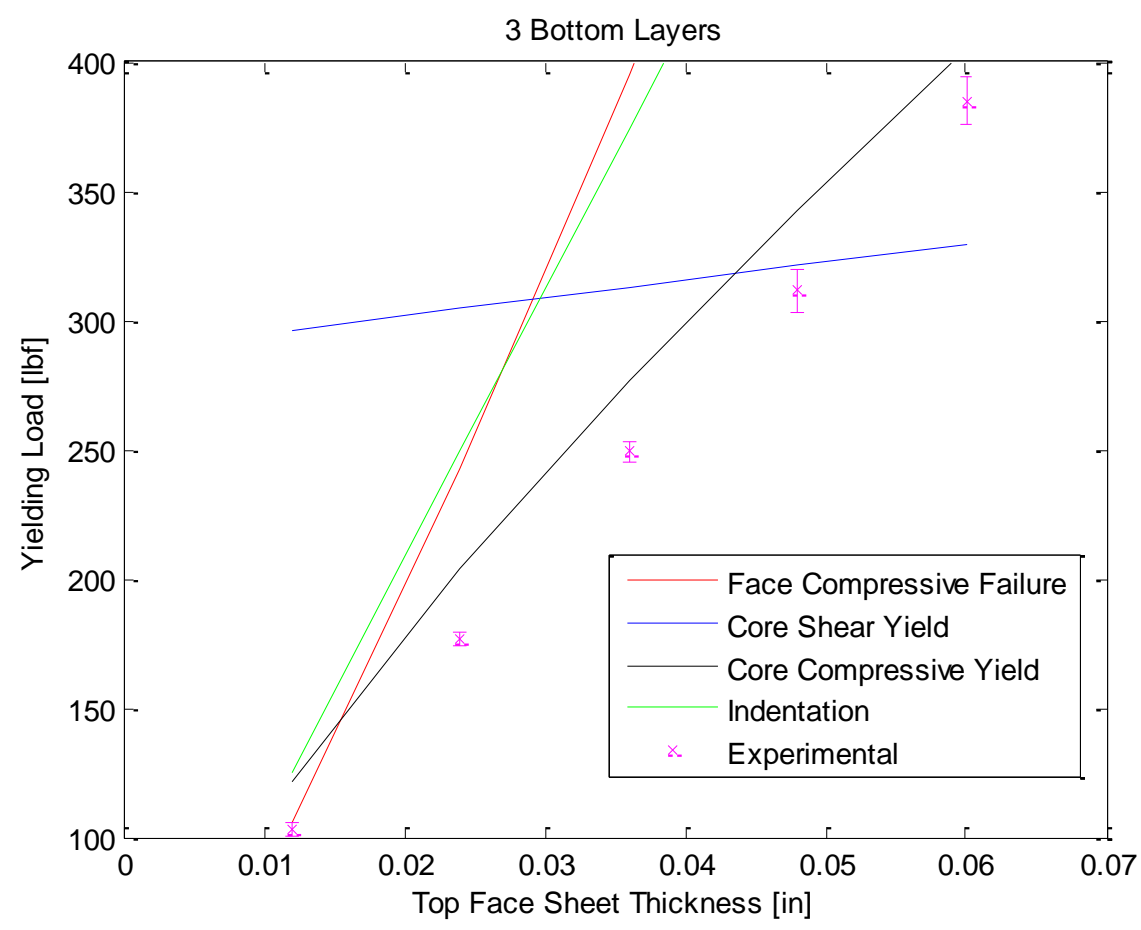


Figure 6.3.A Varying Top Face Sheet thicknesses Failure Loads for 4 Bottom Layers

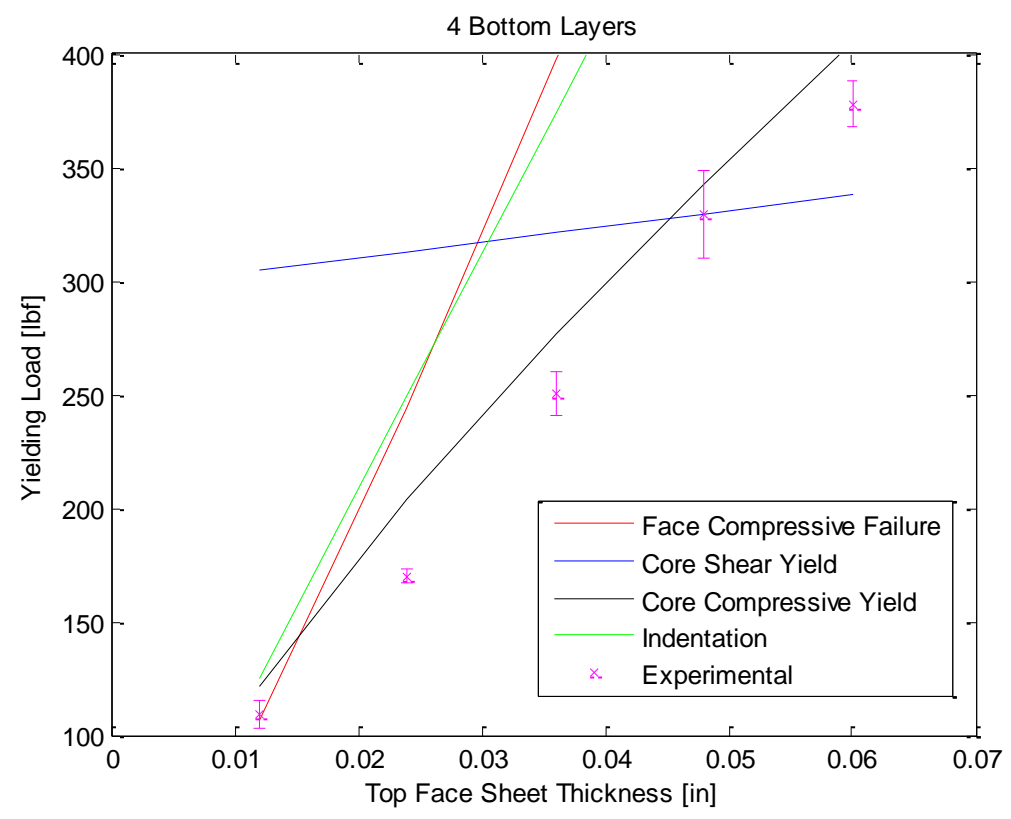

Figure 6.4.A Varying Top Face Sheet thicknesses Failure Loads for 5 Bottom Layers

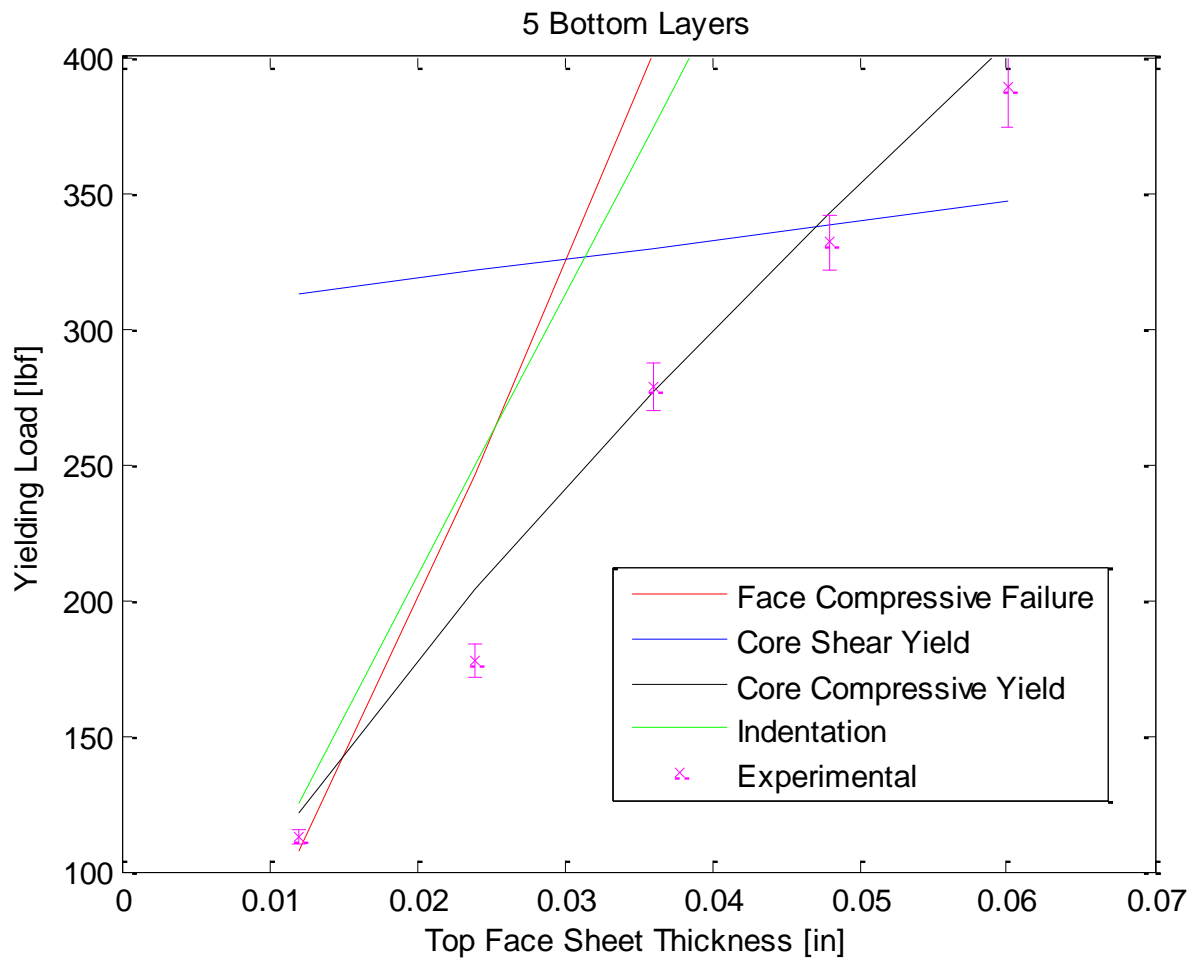




\section{A.7 Varying Face sheet Thickness FEA Plots}

\section{Figures 7.1.A. \& 7.2.A. 4/5 Configuration FEA Stress and Displacements Graphs}
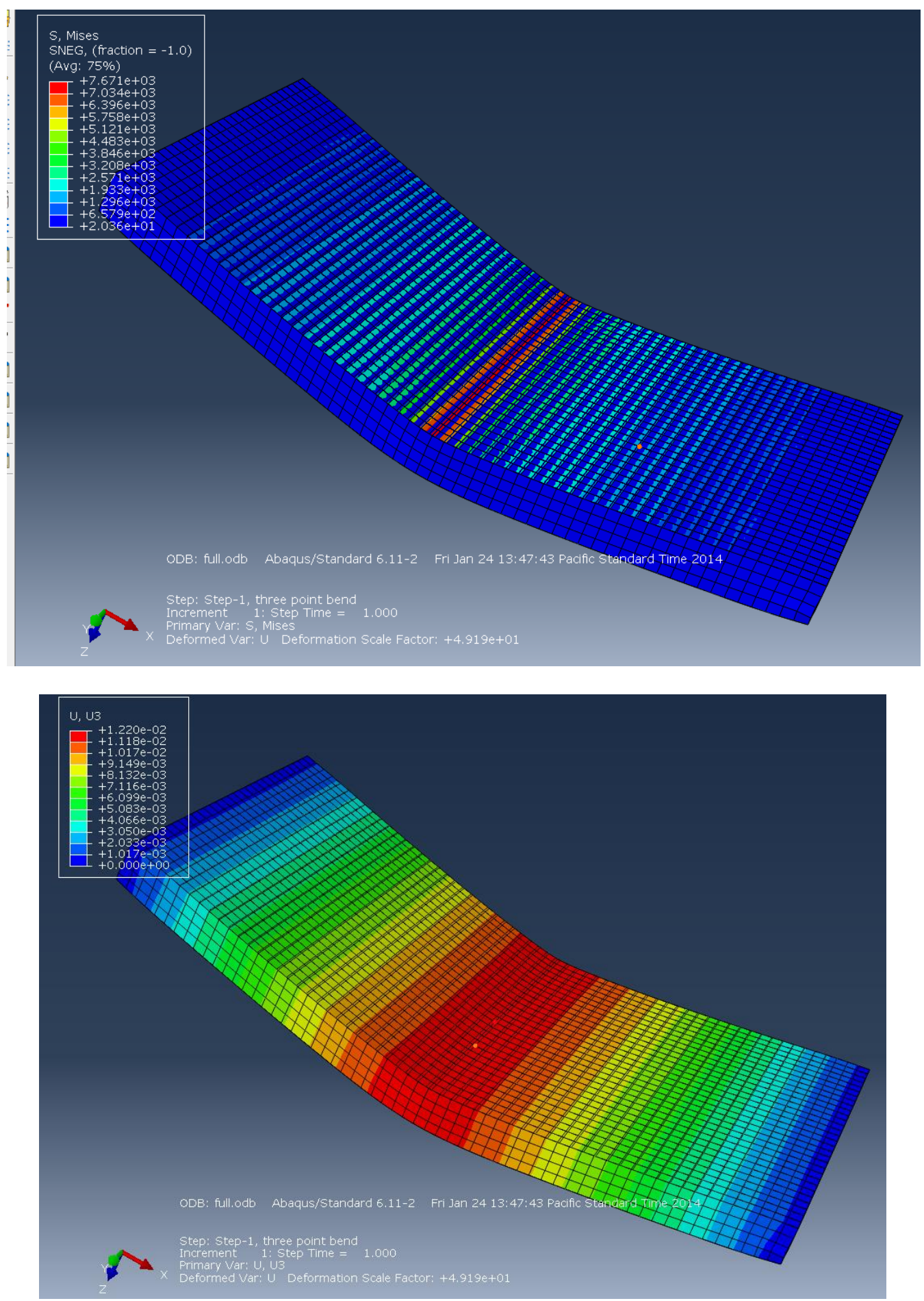
Figures 7.3.A. \& 7.4.A. 3/5 Configuration FEA Stress and Displacements Graphs
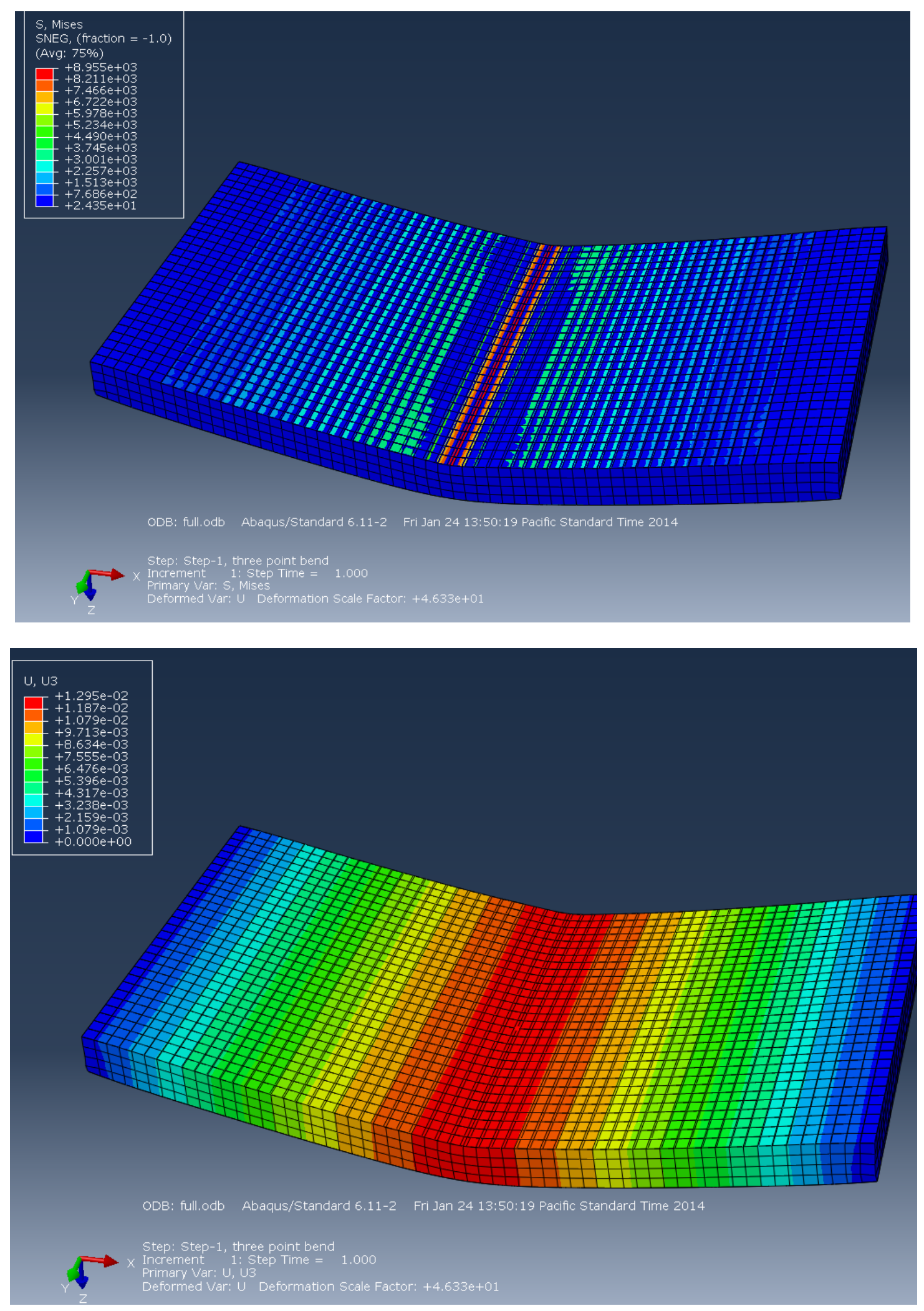
Figures 7.5.A. \& 7.6.A. 2/5 Configuration FEA Stress and Displacements Graphs
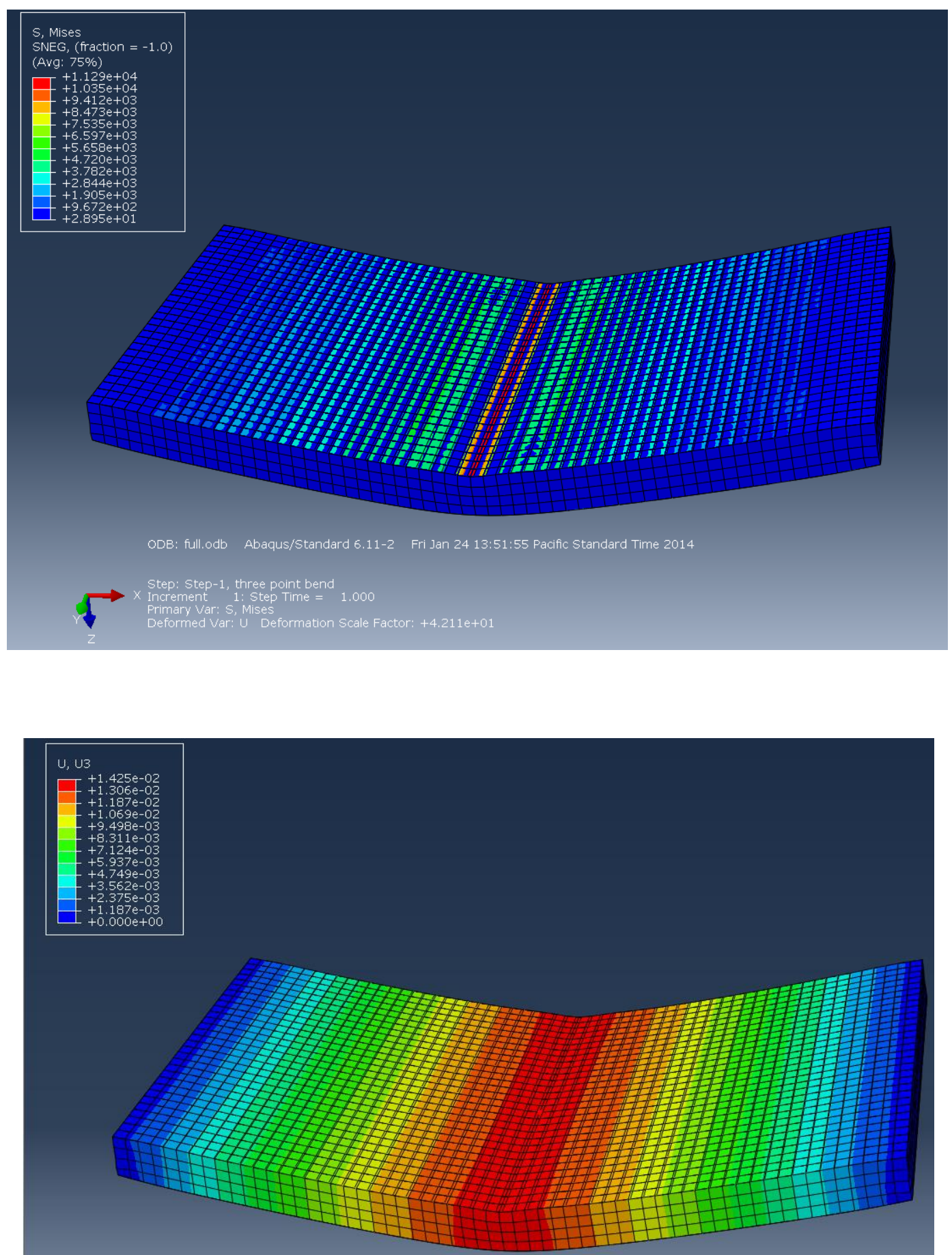

ODB: full.odb Abaqus/Standard 6.11-2 Fri Jan 24 13:51:55 Pacific Standard Time 2014

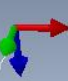

Step: Step-1, three point bend

Primer $1:$ Step Time $=1.000$

Deformed var: $U$ Deformation Scale Factor: $+42110+01$ 
Figures 7.7.A. \& 7.8.A. 1/5 Configuration FEA Stress and Displacements Graphs
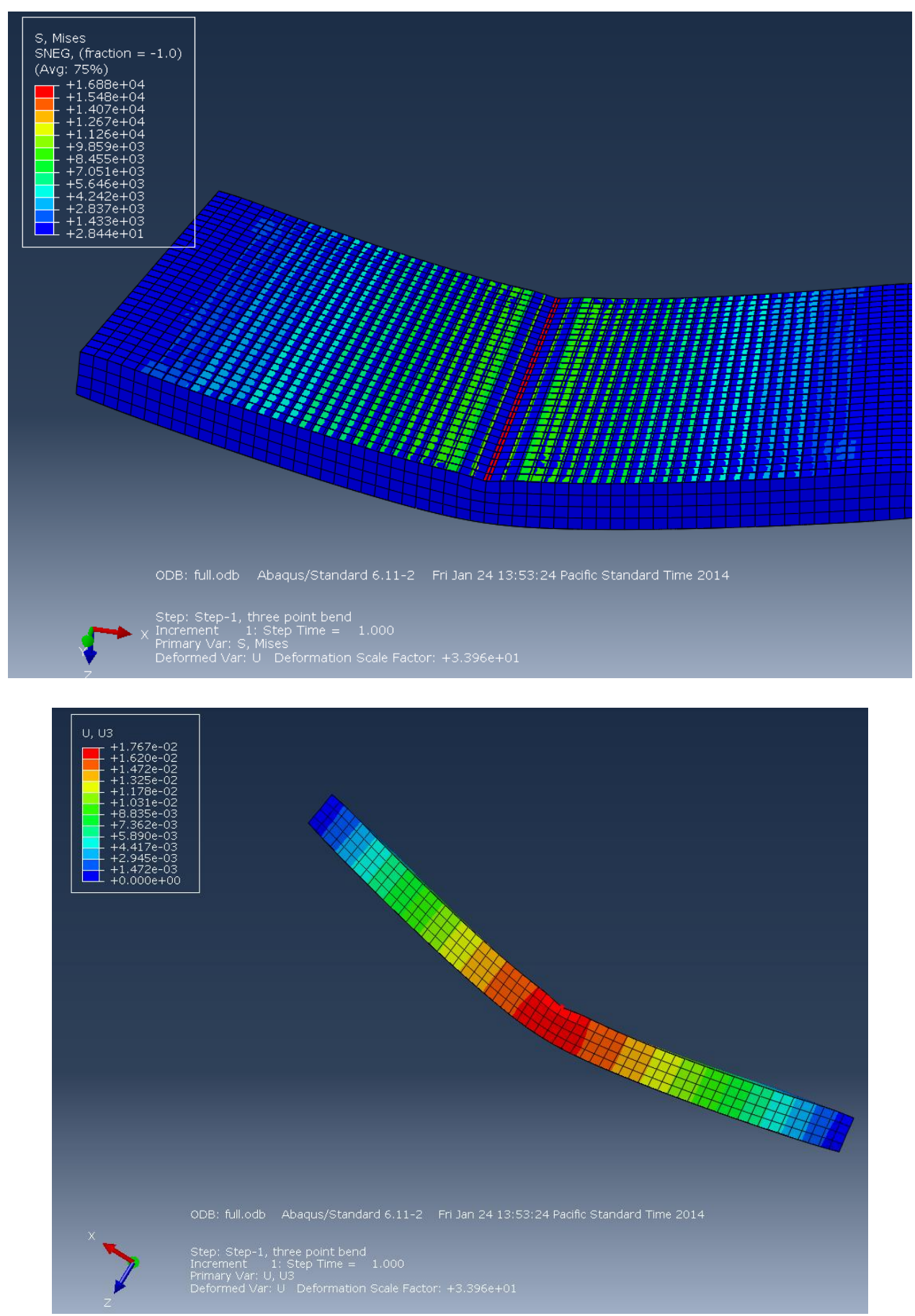
Figures 7.9.A. \& 7.10.A. 1/4 Configuration FEA Stress and Displacements Graphs
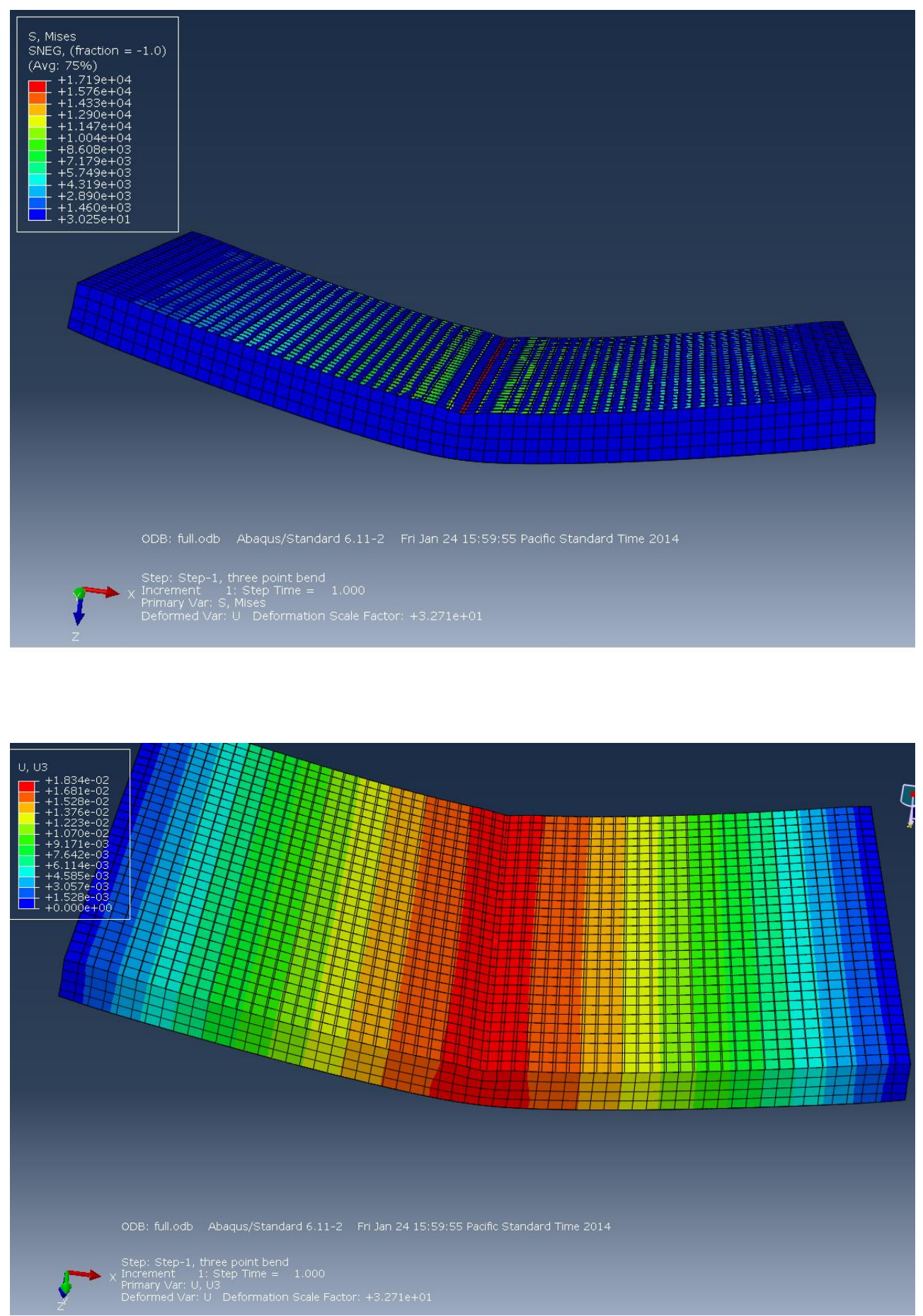
Figures 7.11.A. \& 7.12.A. 2/4 Configuration FEA Stress and Displacements Graphs
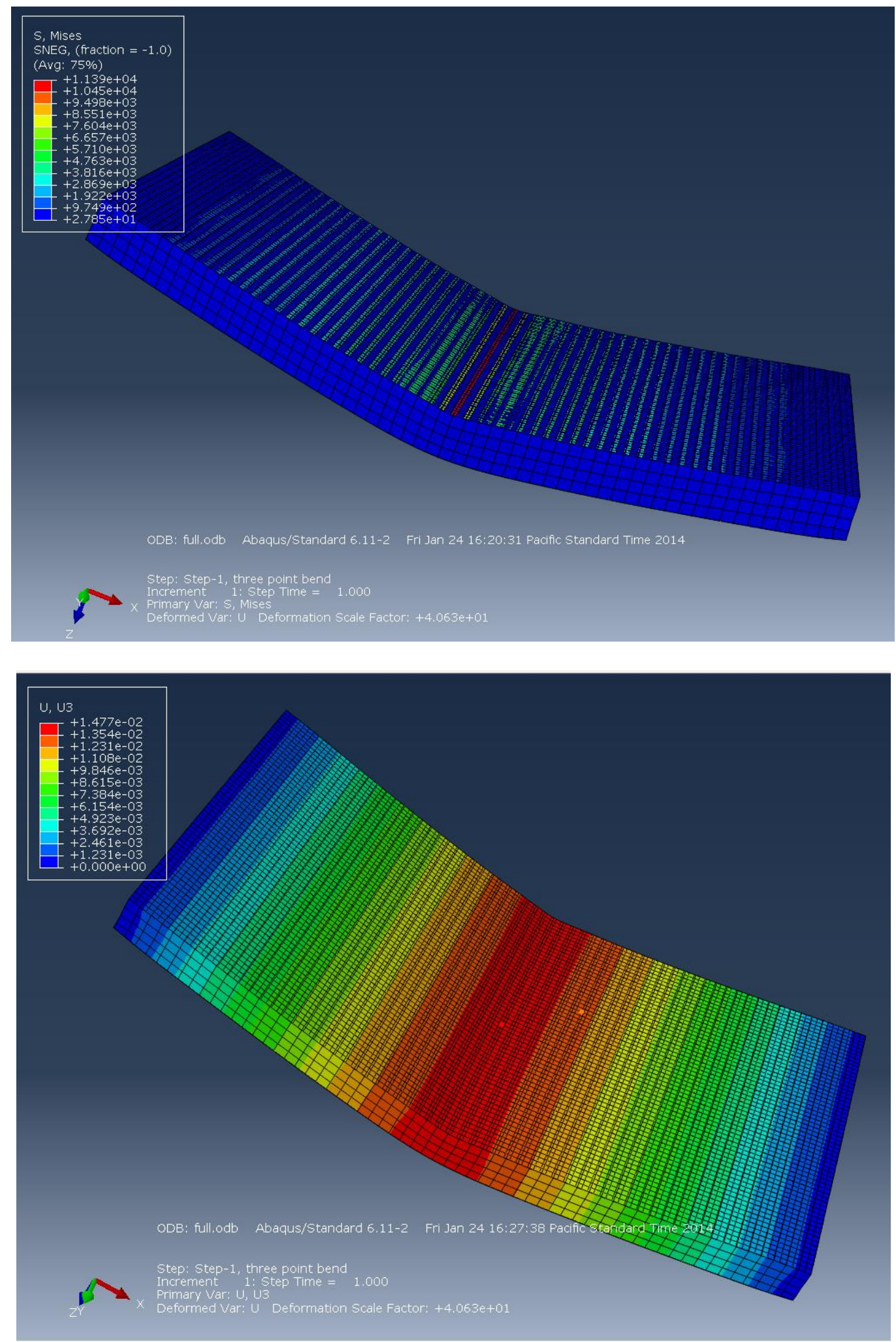
Figures 7.13.A. \& 7.14.A. 3/4 Configuration FEA Stress and Displacements Graphs
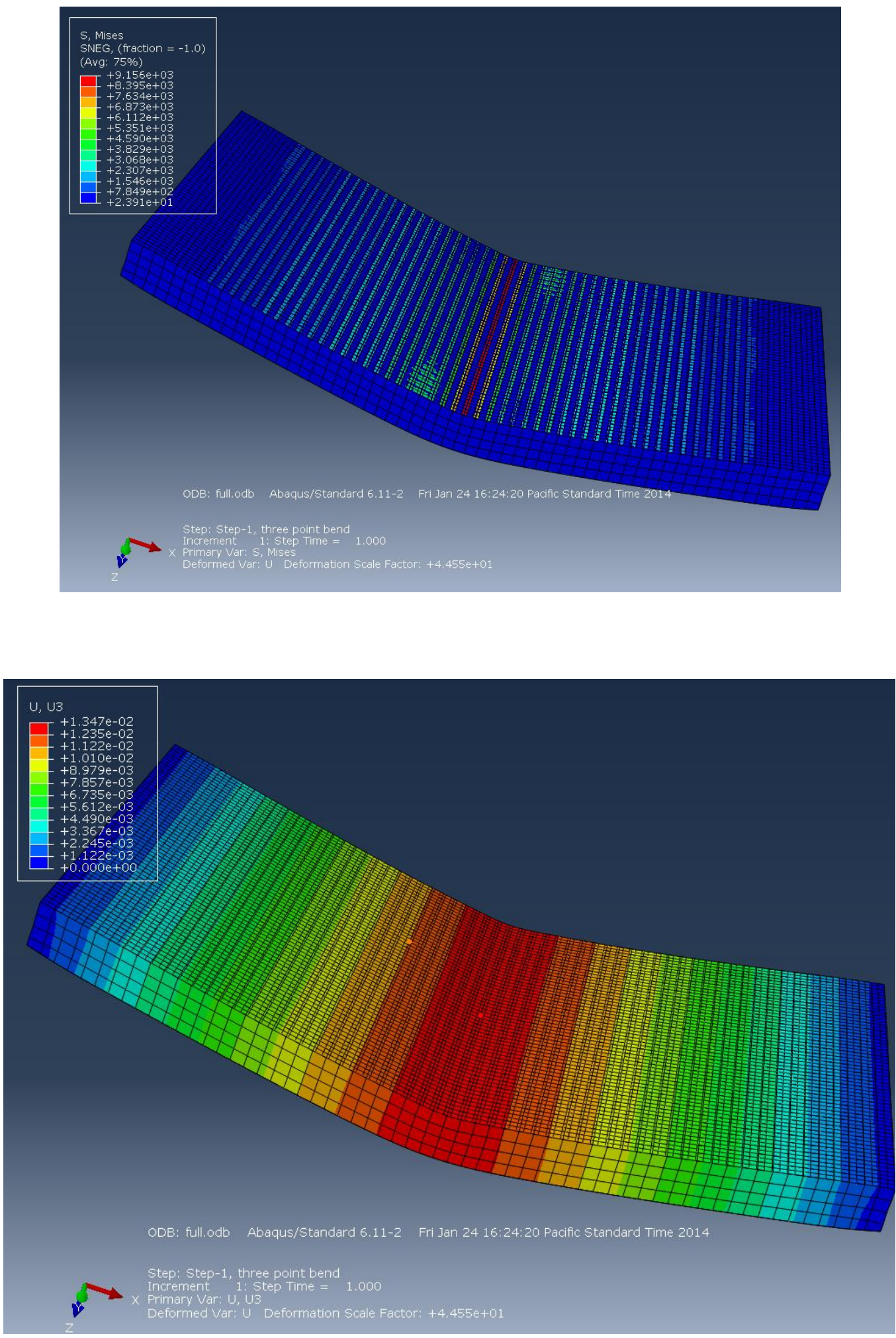
Figures 7.15.A. \& 7.16.A. 4/4 Configuration FEA Stress and Displacements Graphs
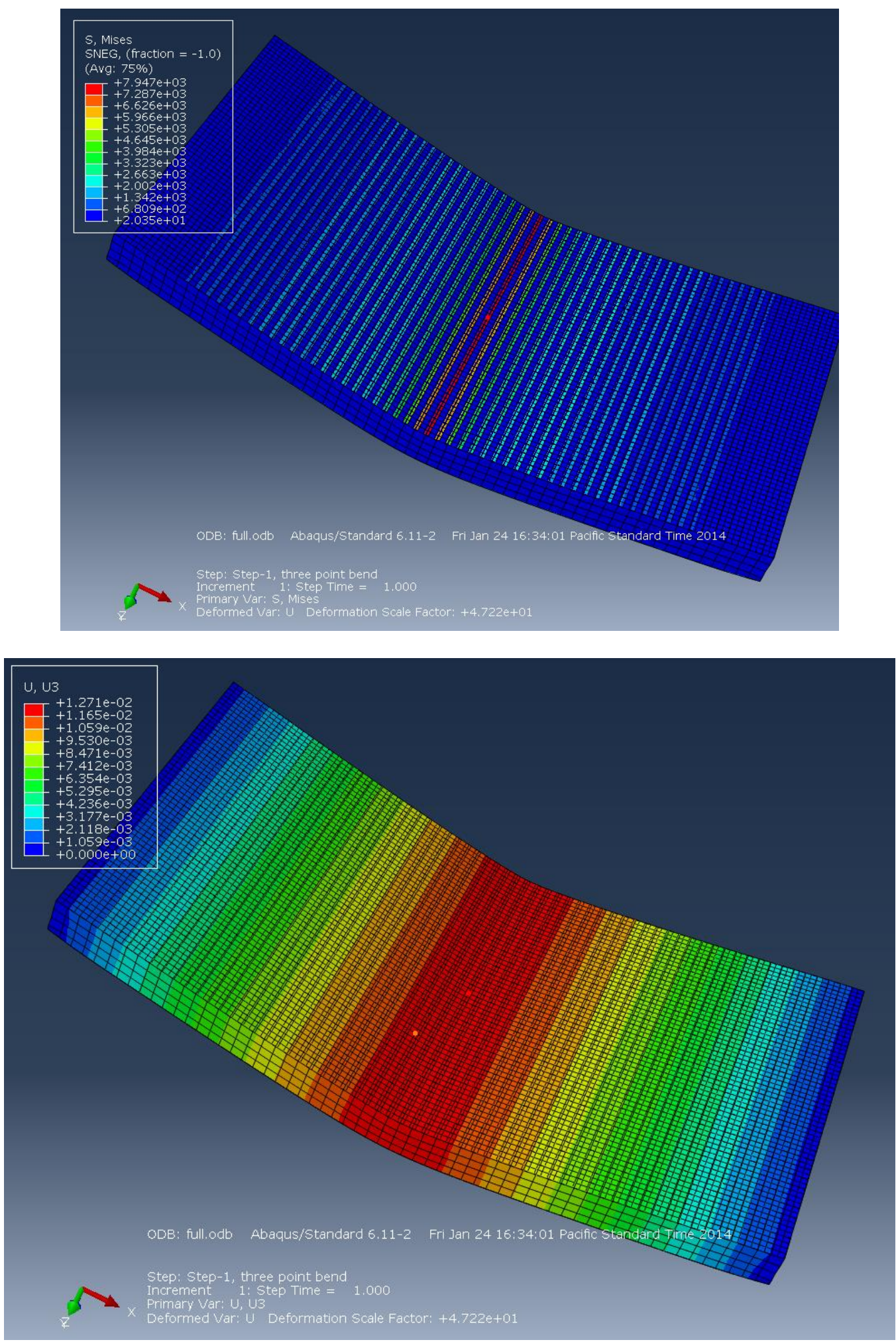
Figures 7.17.A. \& 7.18.A. 5/4 Configuration FEA Stress and Displacements Graphs
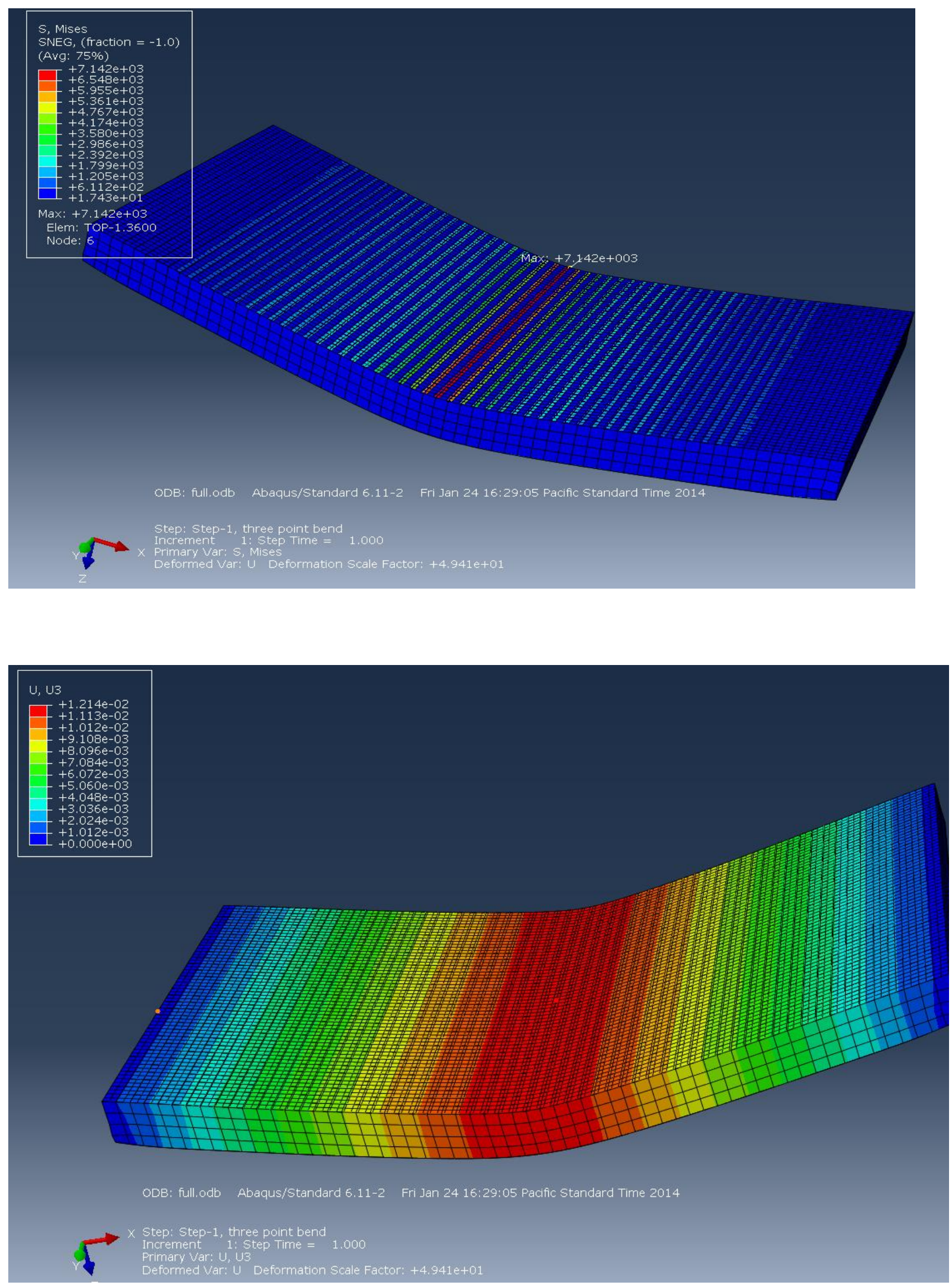
Figures7.19.A. \& 7.20.A. 5/3 Configuration FEA Stress and Displacements Graphs
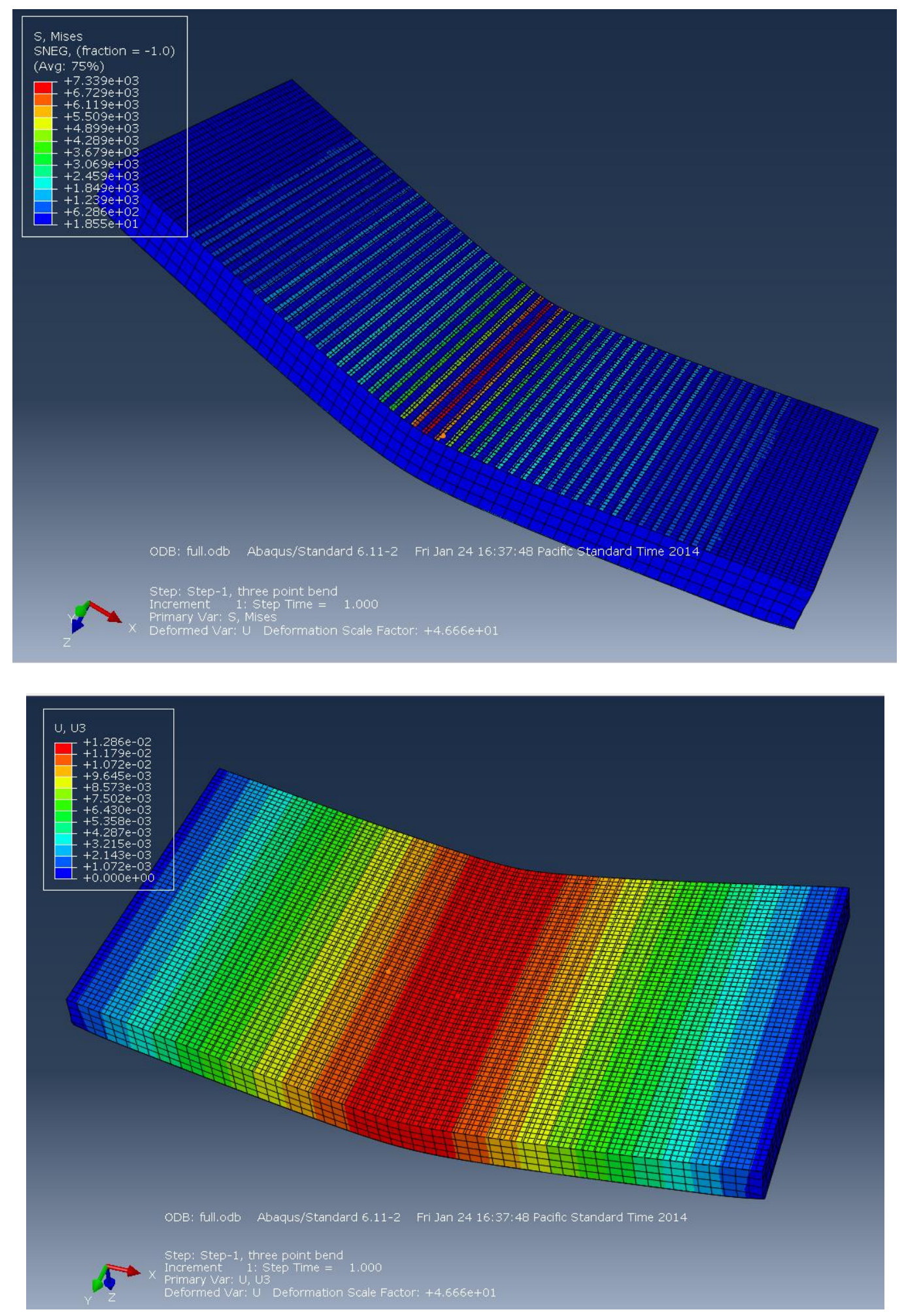
Figures 7.21.A. \& 7.22.A 4/3 Configuration FEA Stress and Displacements Graphs
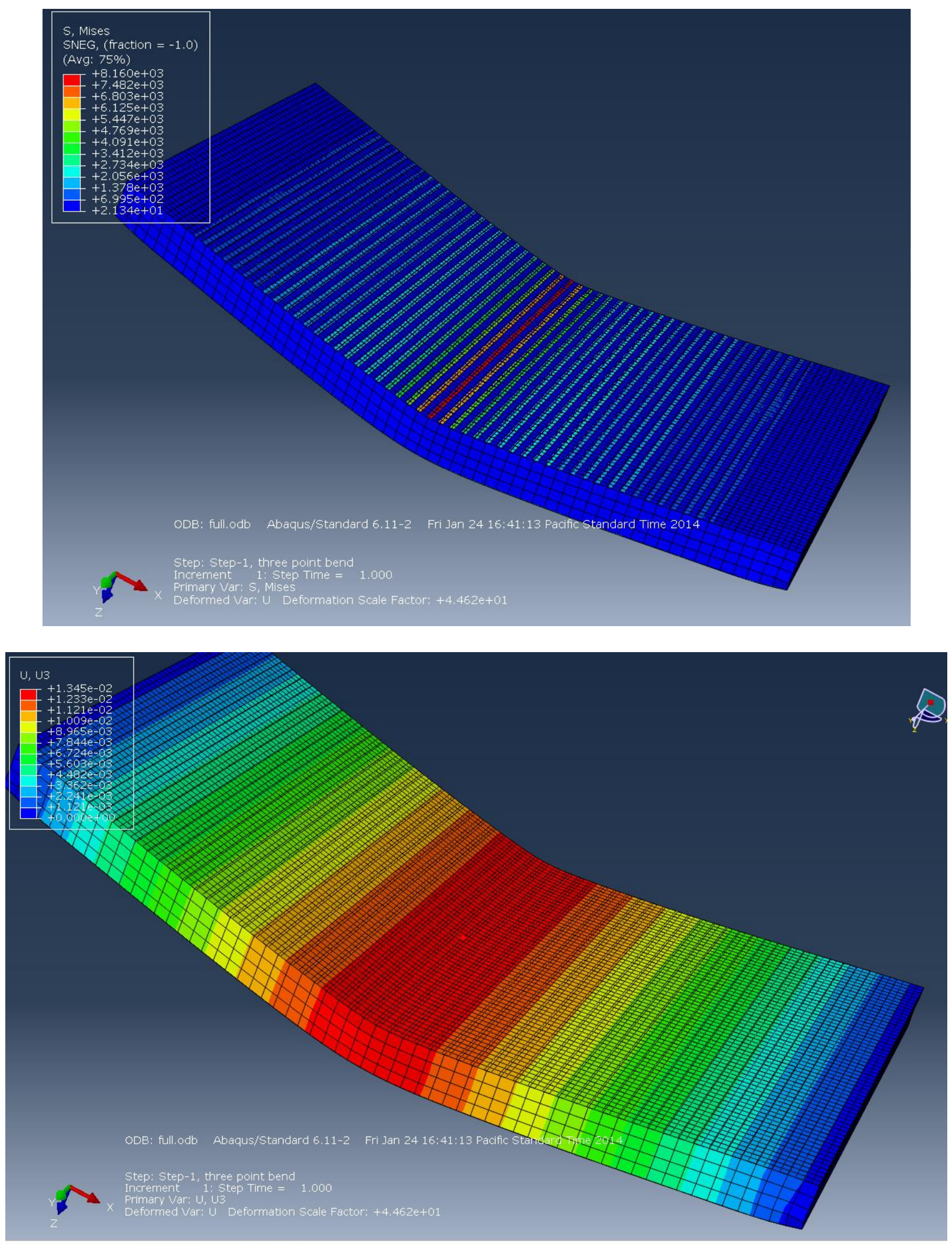
Figures 7.23.A. \& 7.24.A. 3/3 Configuration FEA Stress and Displacements Graphs
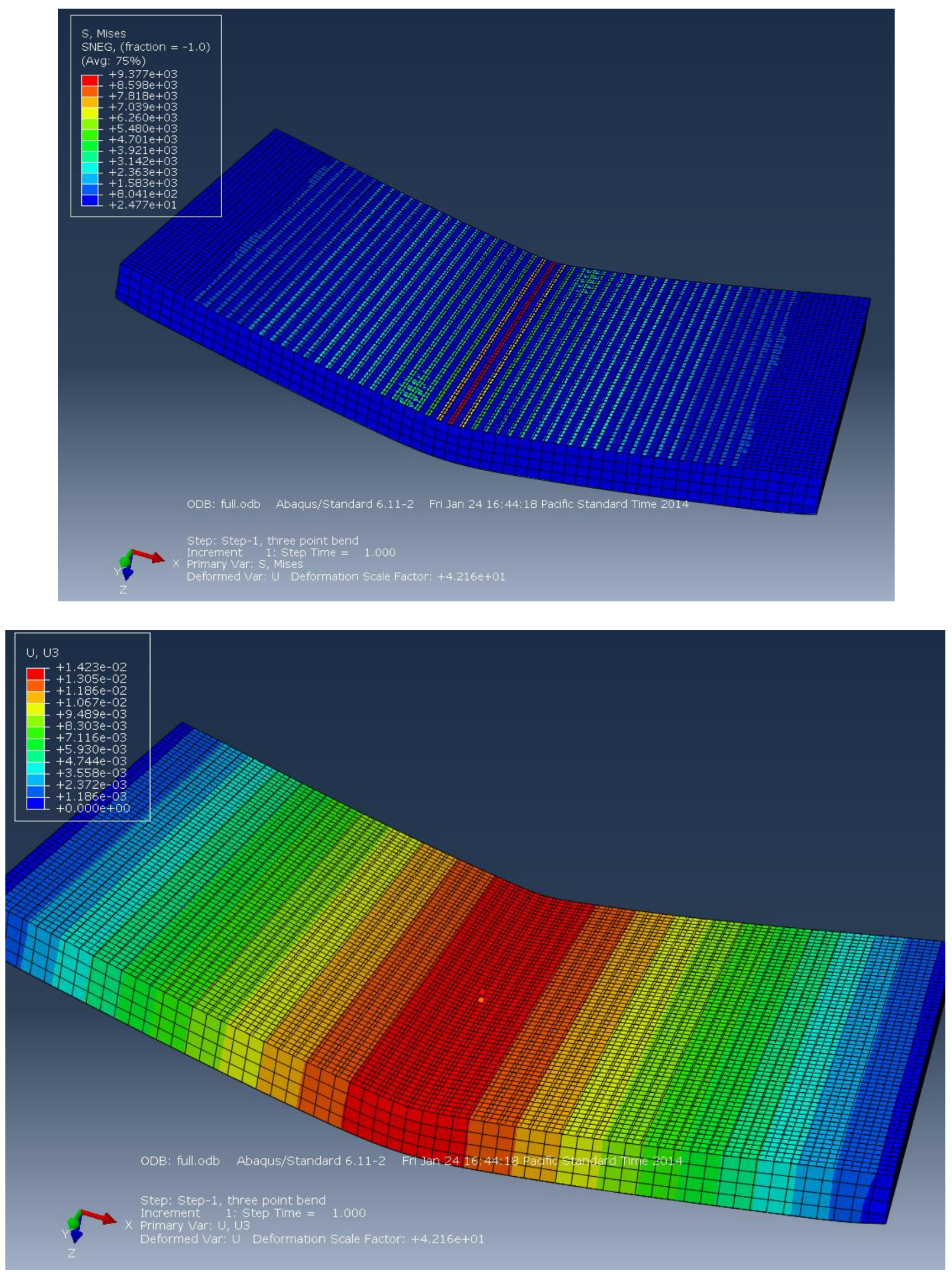
Figures 7.25.A. \& 7.26.A. 2/3 Configuration FEA Stress and Displacements Graphs
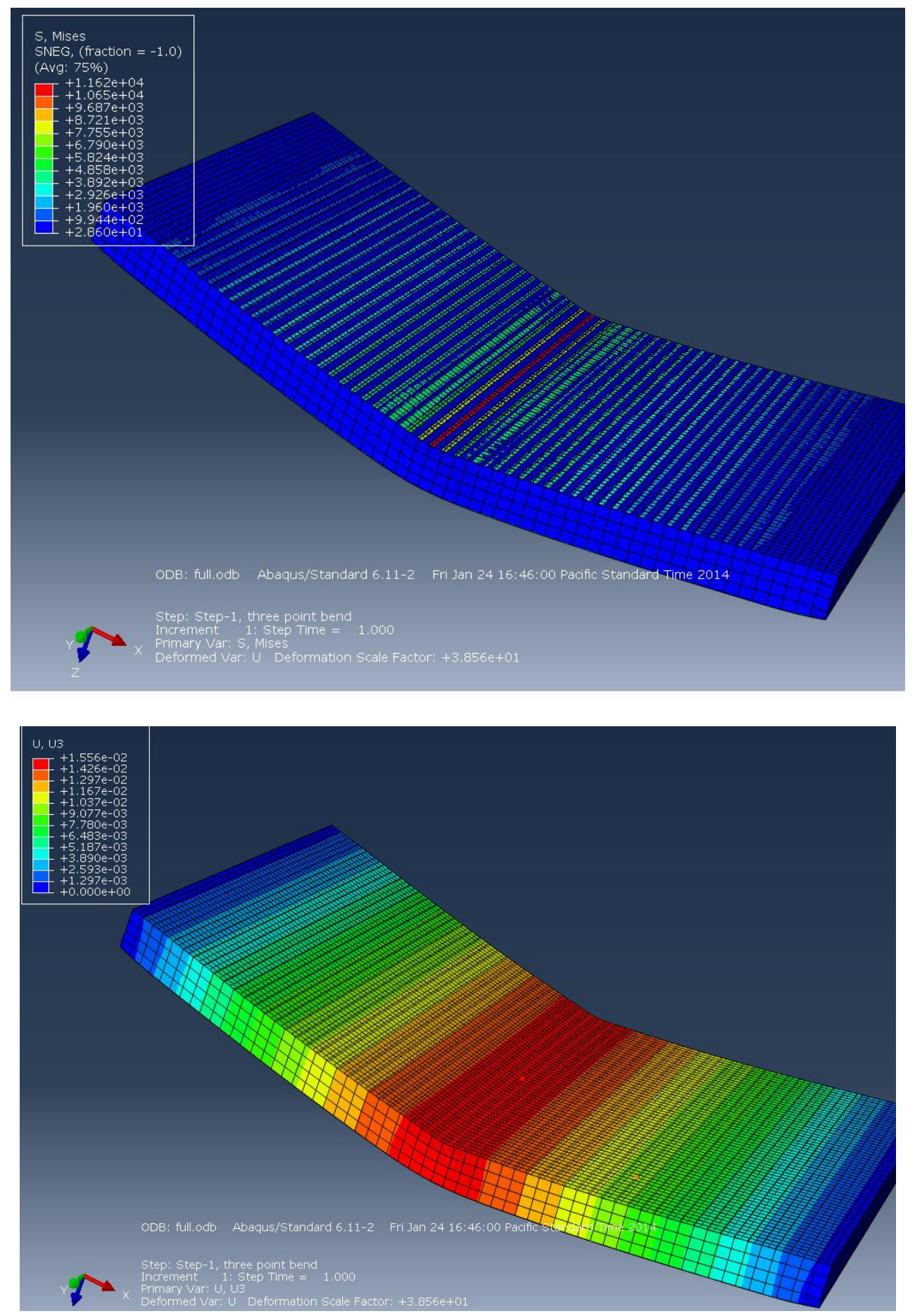
Figures 7.27.A. \& 7.28.A. 1/3 Configuration FEA Stress and Displacements Graphs
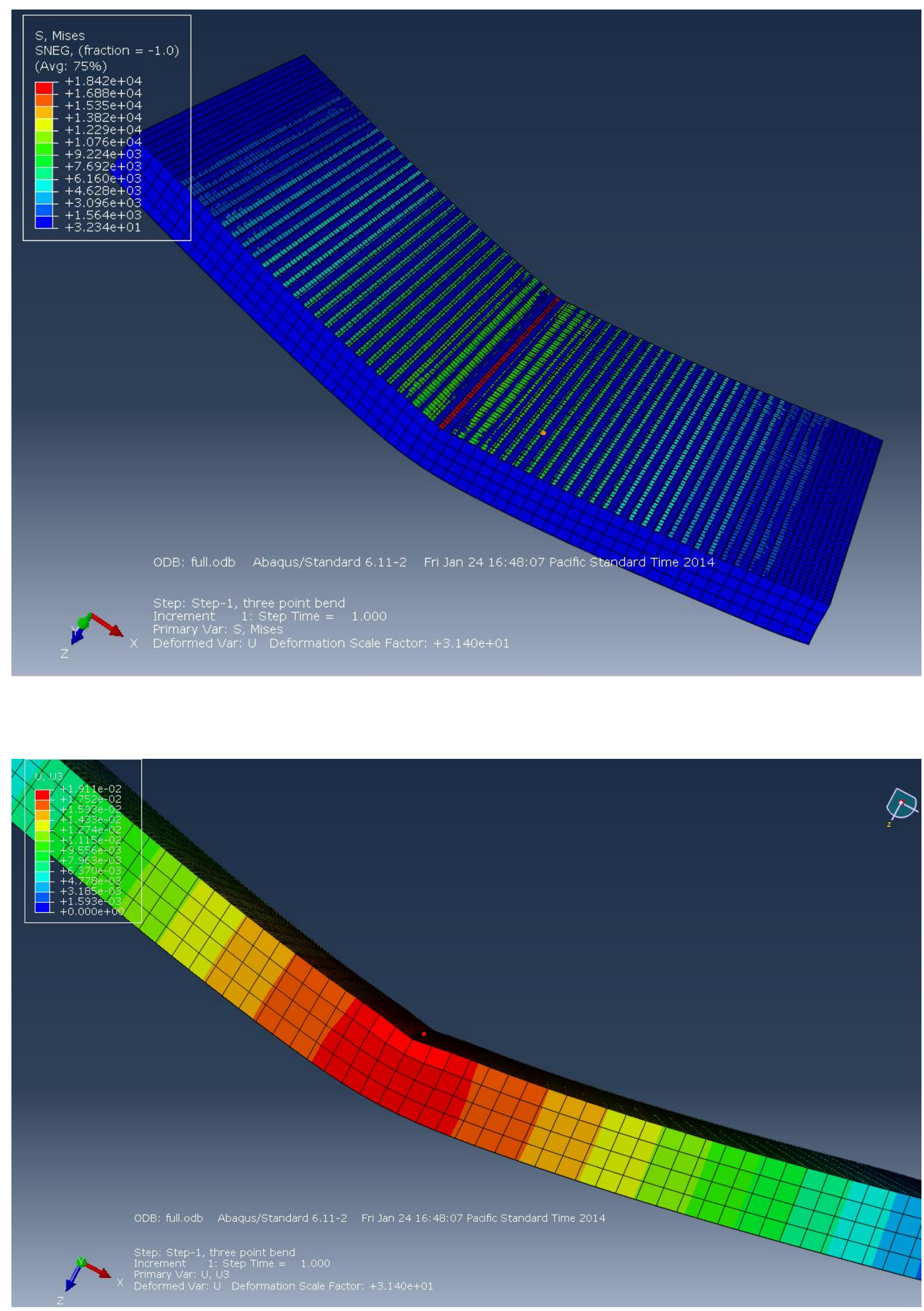
Figures 7.29.A. \& 7.30.A. 5/2 Configuration FEA Stress and Displacements Graphs
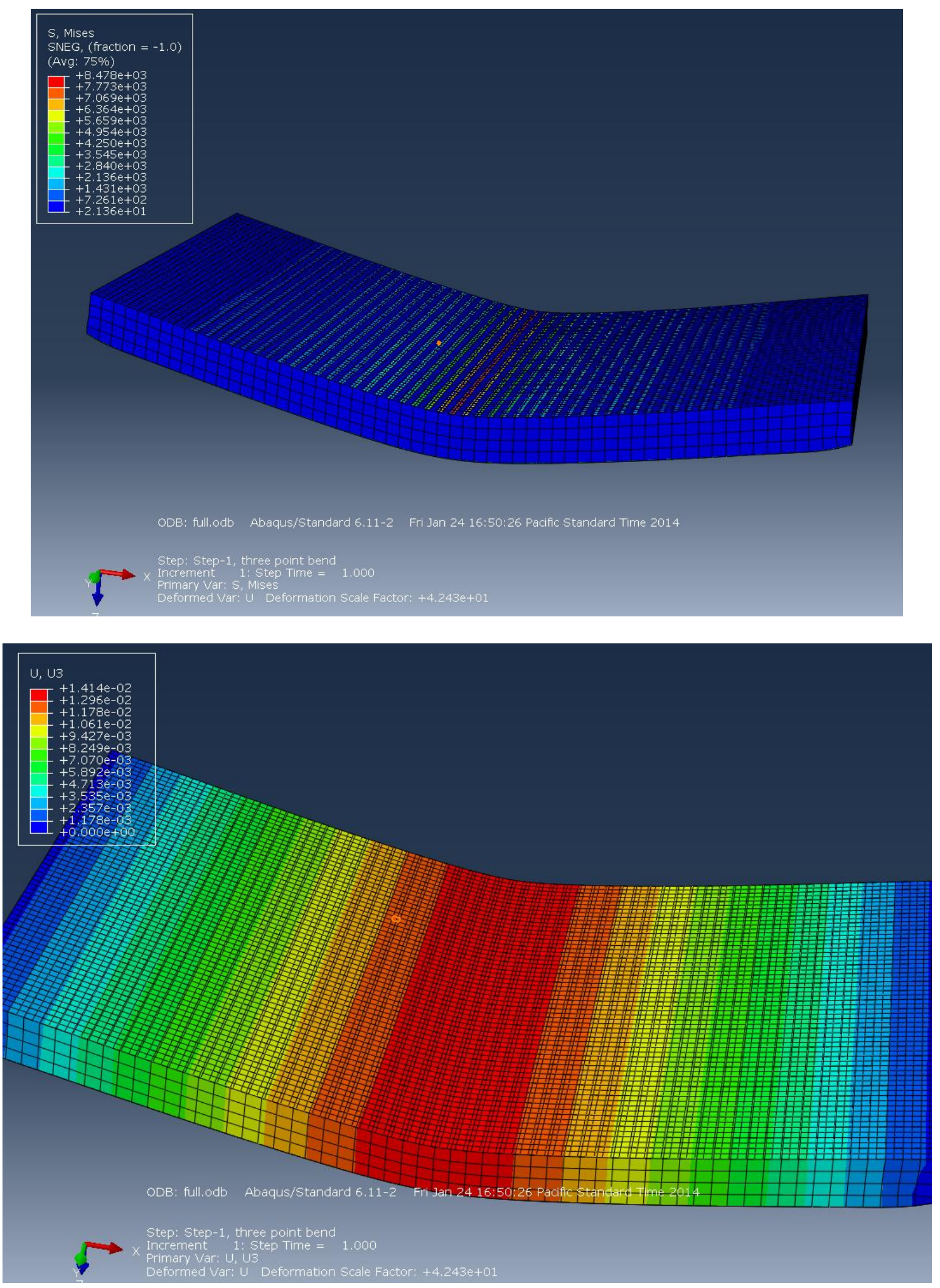
Figures 7.31.A. \& 7.32.A. 4/2 Configuration FEA Stress and Displacements Graphs
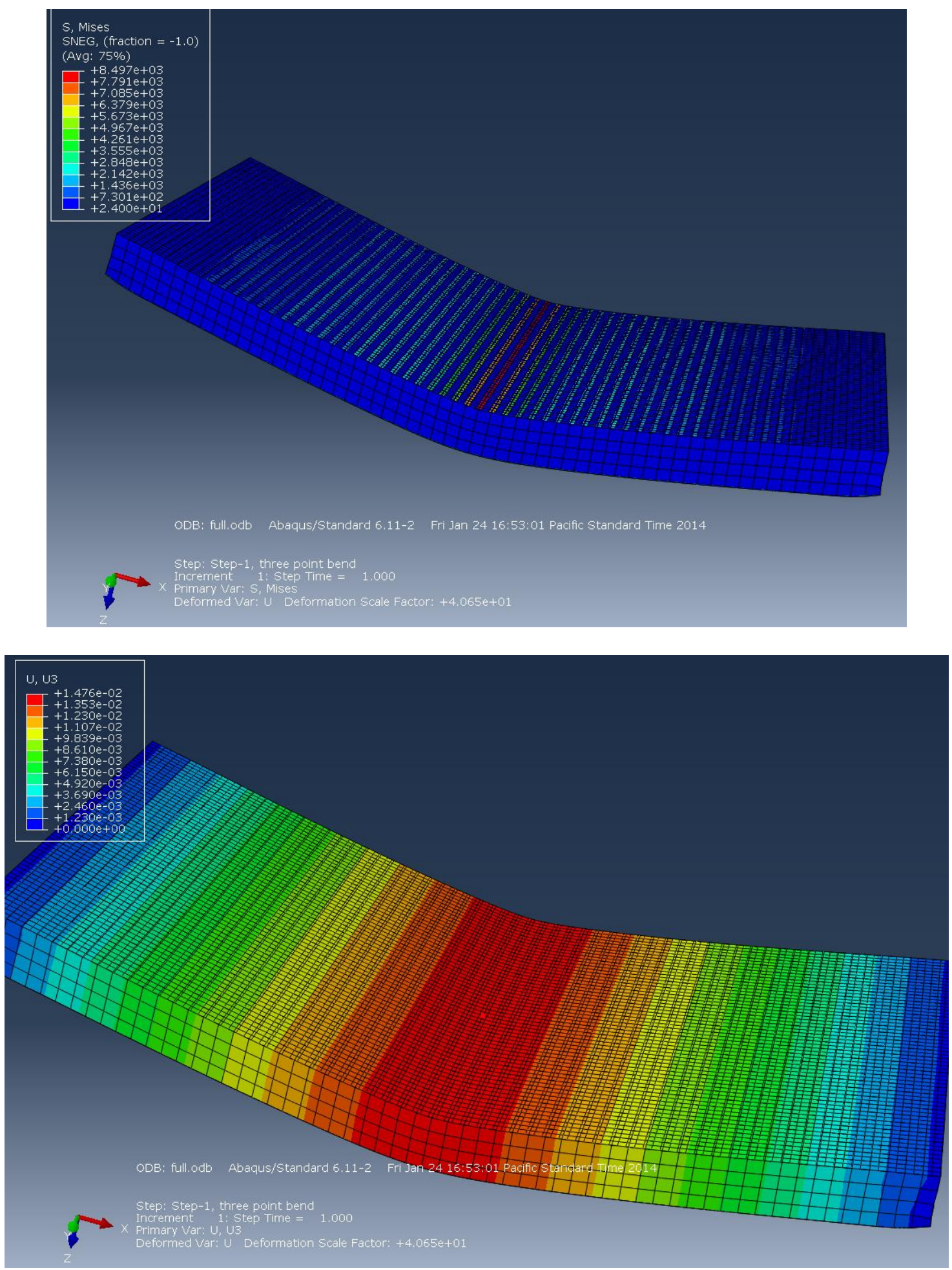
Figures 7.33.A. \& 7.34.A. 3/2 Configuration FEA Stress and Displacements Graphs
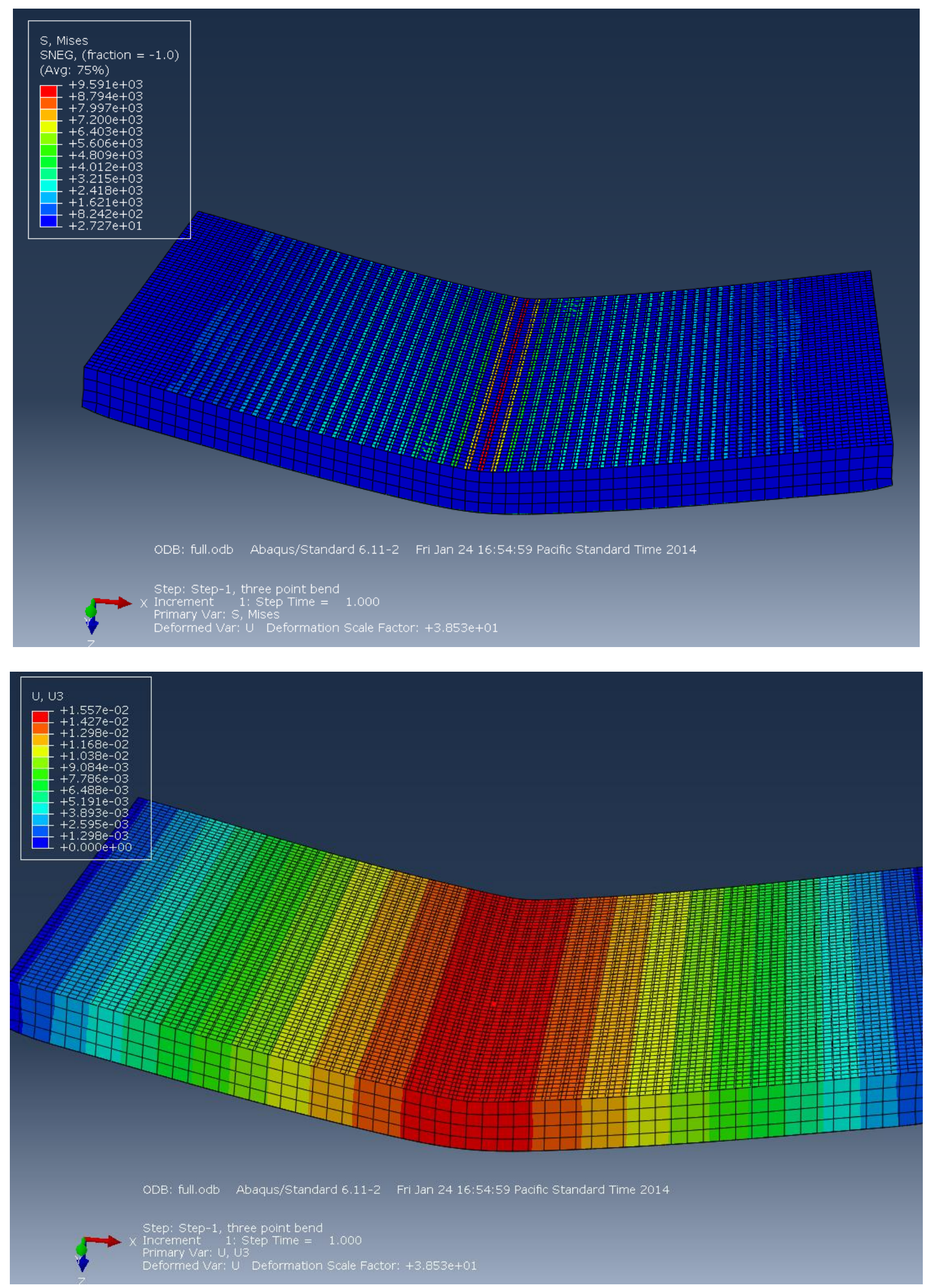
Figures 7.35.A. \& 7.36.A. 2/2 Configuration FEA Stress and Displacements Graphs
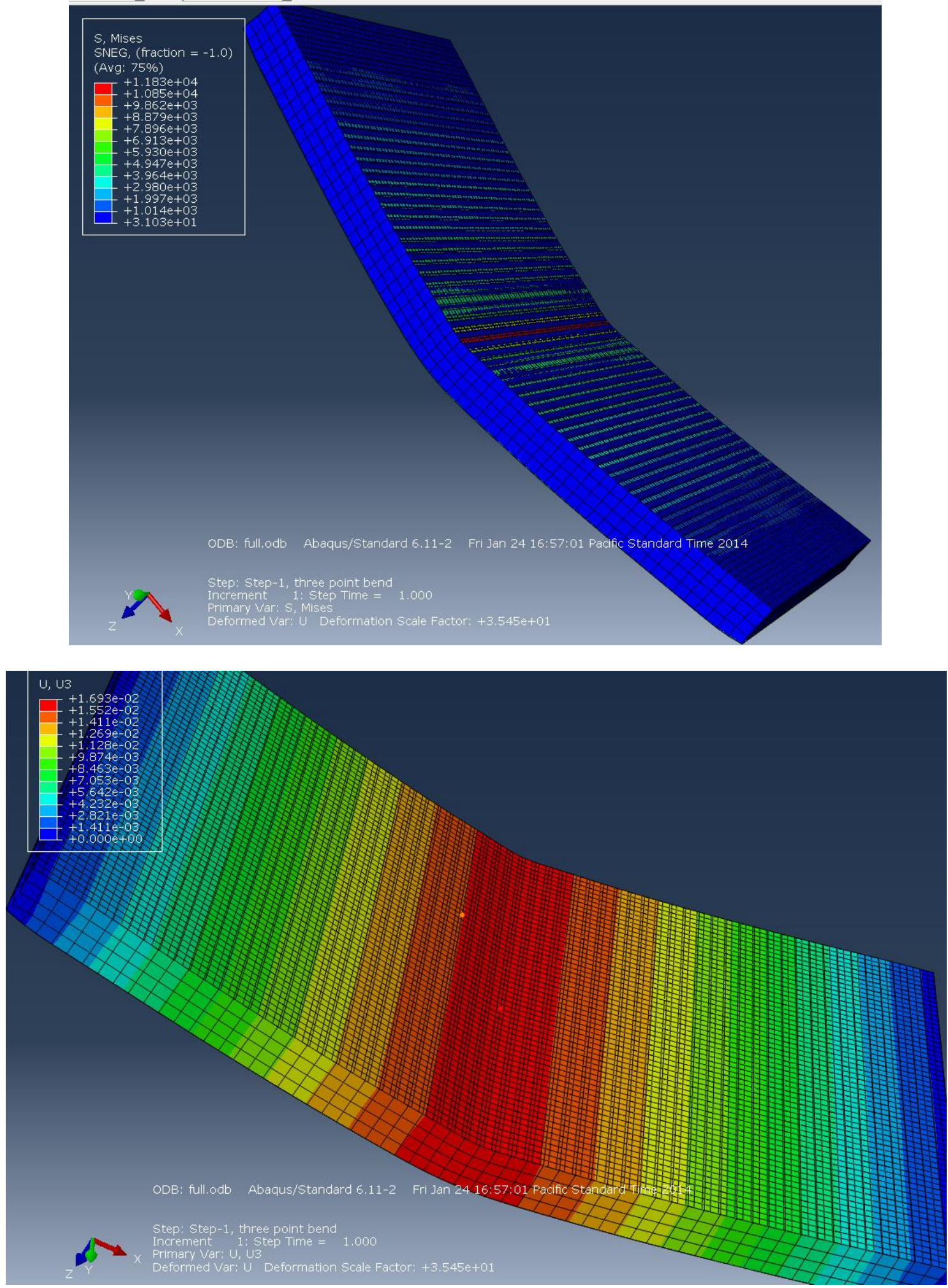
Figures 7.37.A. \& 7.38.A. 1/2 Configuration FEA Stress and Displacements Graphs
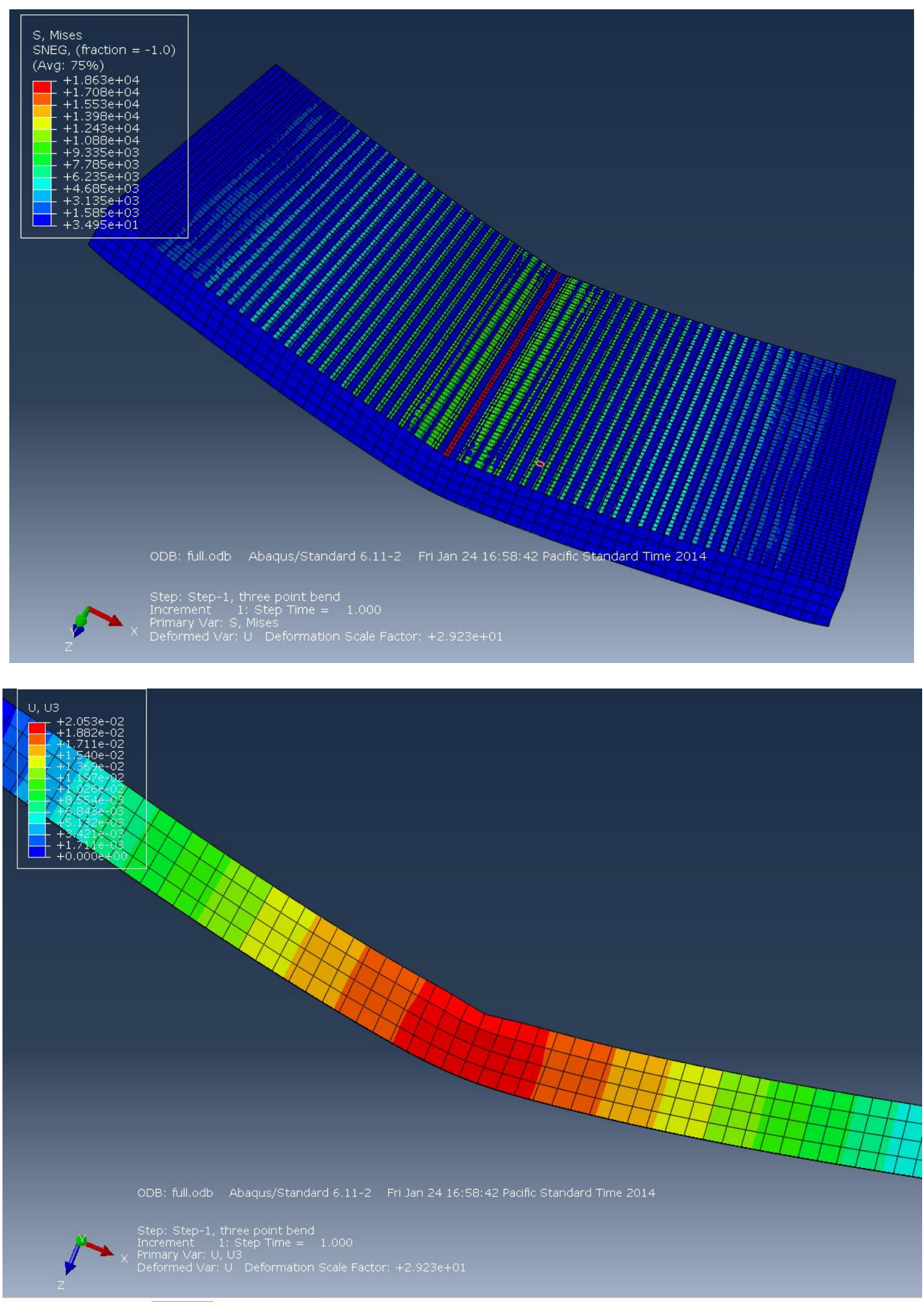
Figures 7.39.A. \& 7.40.A. 5/1 Configuration FEA Stress and Displacements Graphs
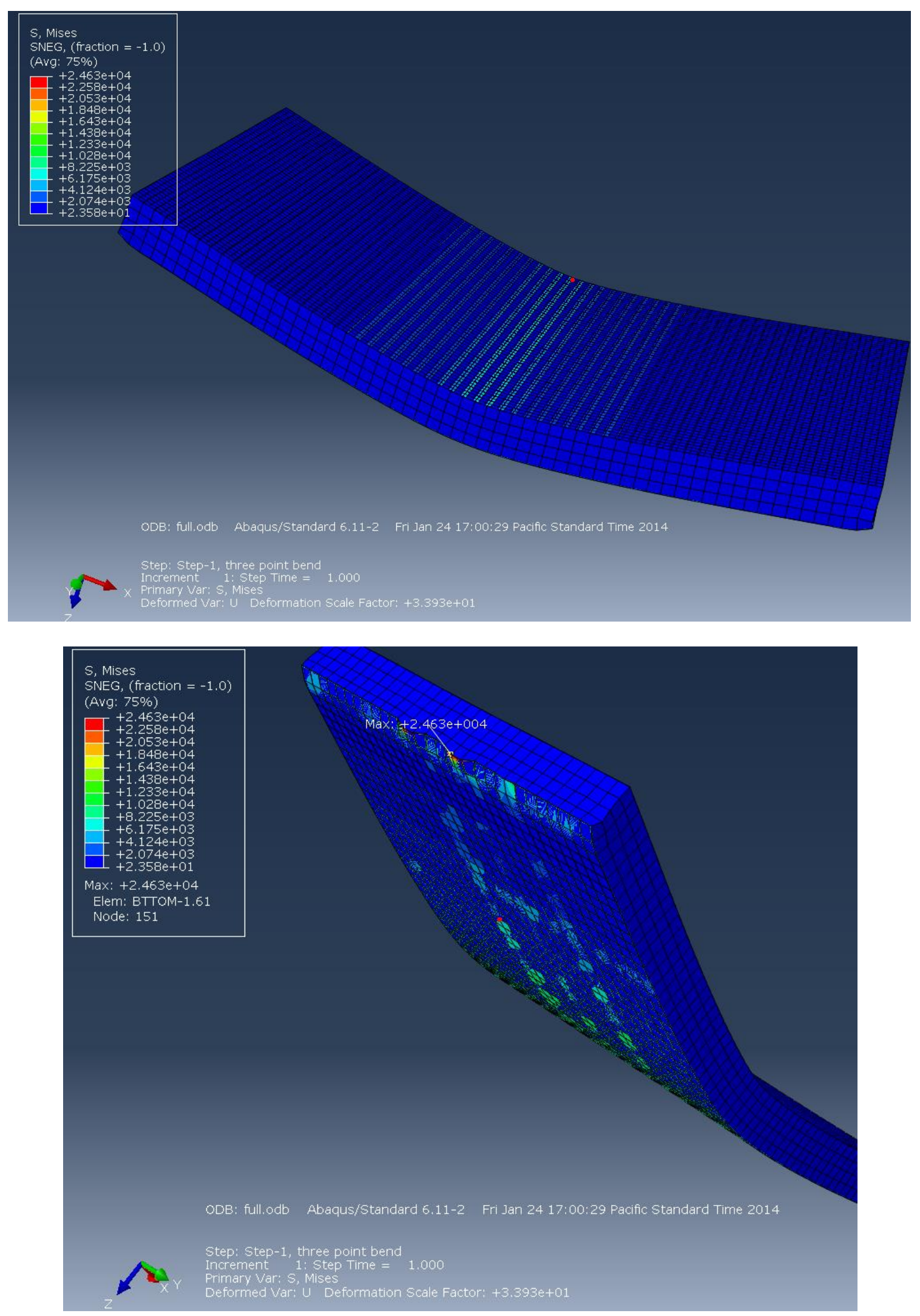
Figures 7.41.A. \& 7.42.A. 4/1 Configuration FEA Stress and Displacements Graphs
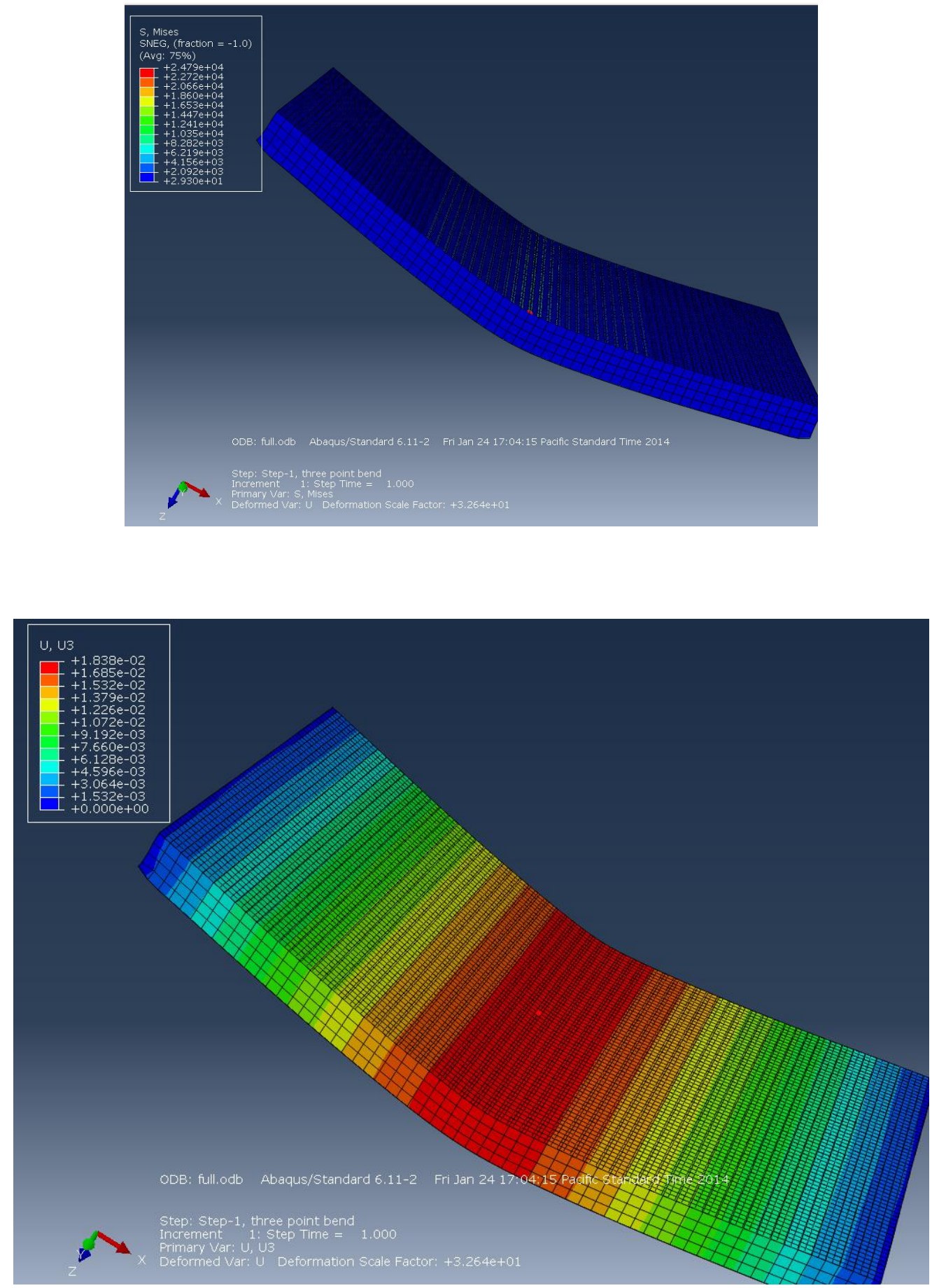
Figures 7.43.A. \& 7.44.A. 3/1 Configuration FEA Stress and Displacements Graphs
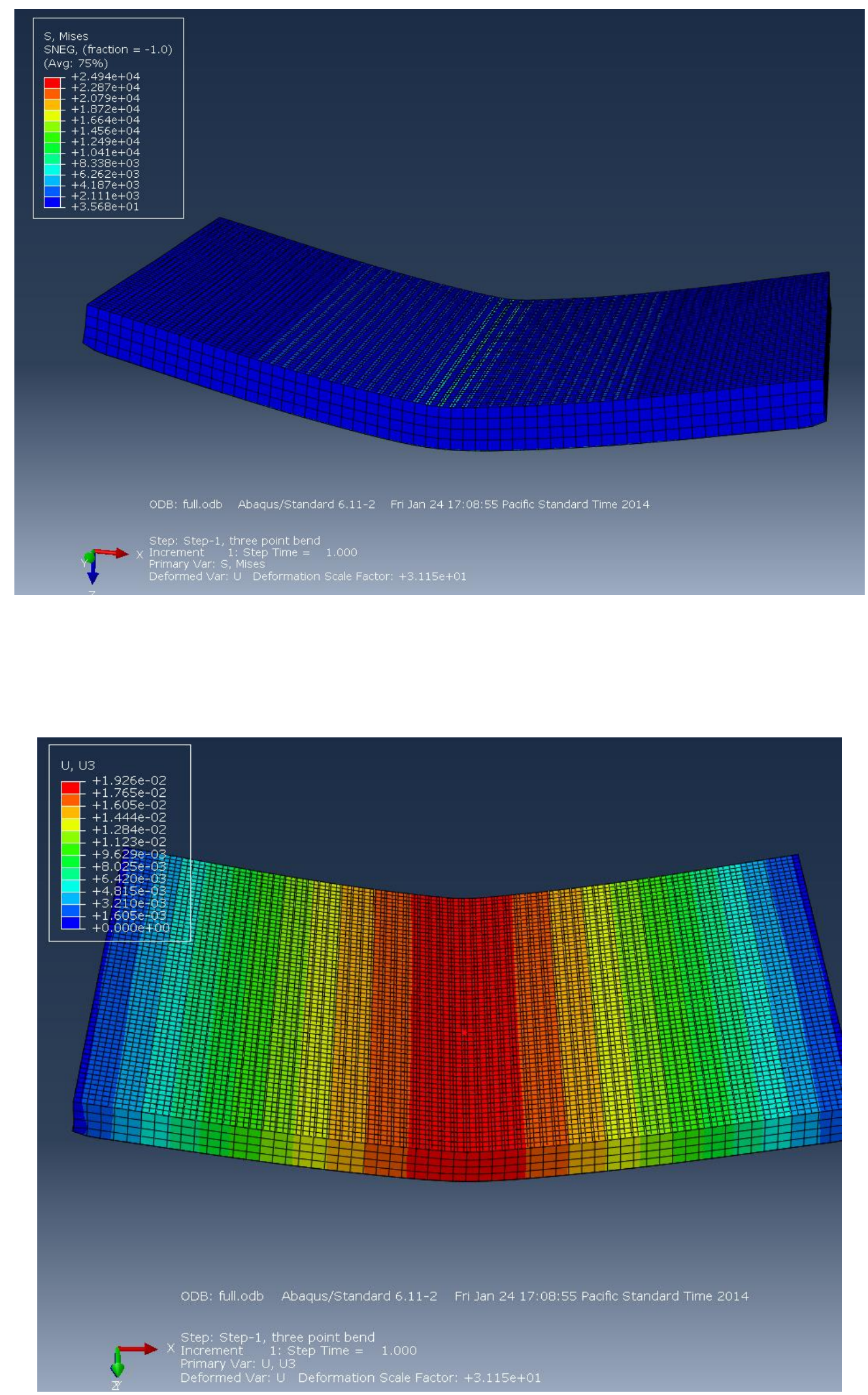
Figures 7.45.A. \& 7.46.A. 2/1 Configuration FEA Stress and Displacements Graphs
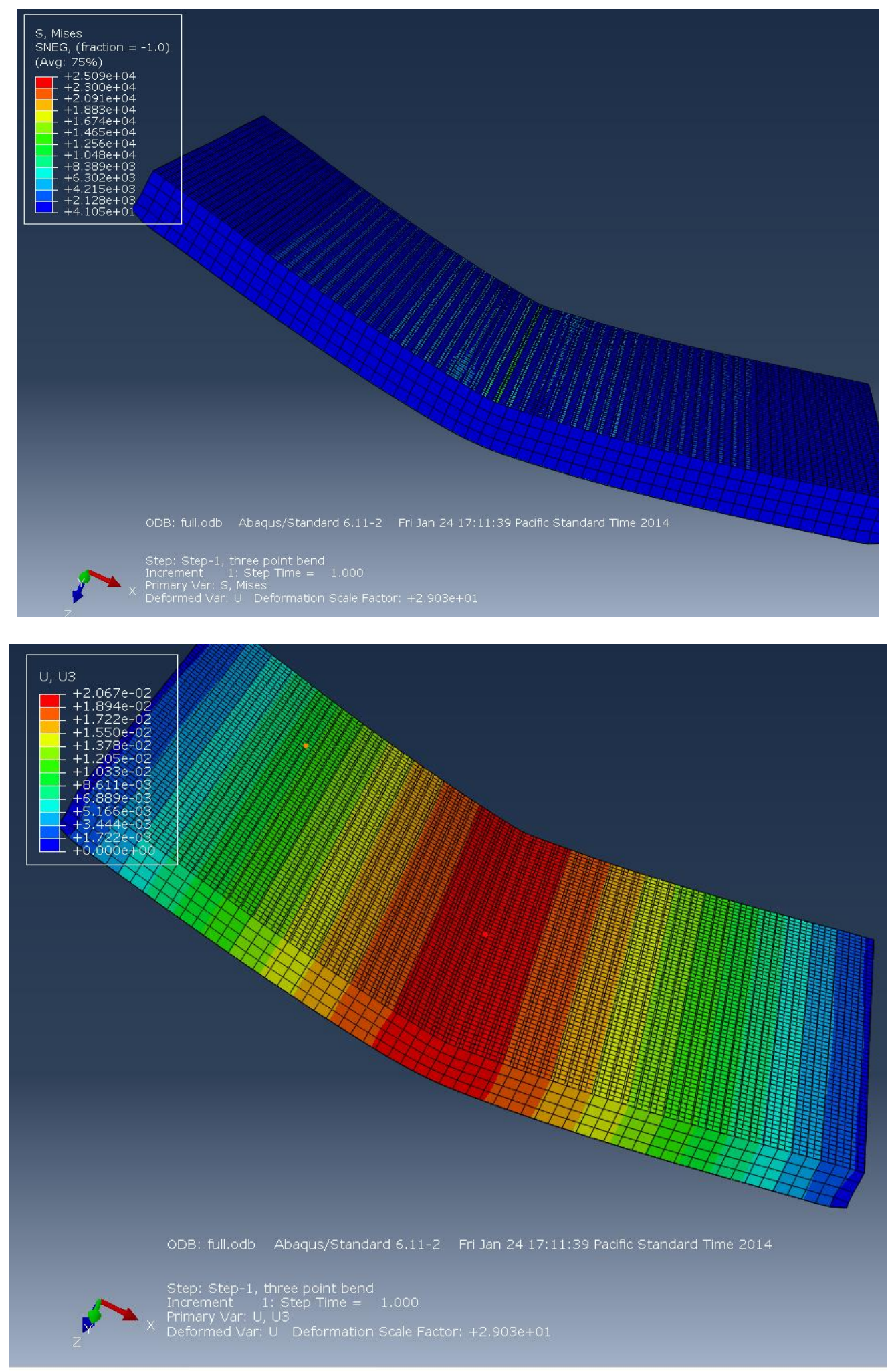
Figures 7.47.A. \& 7.48.A. 1/1 Configuration FEA Stress and Displacements Graphs
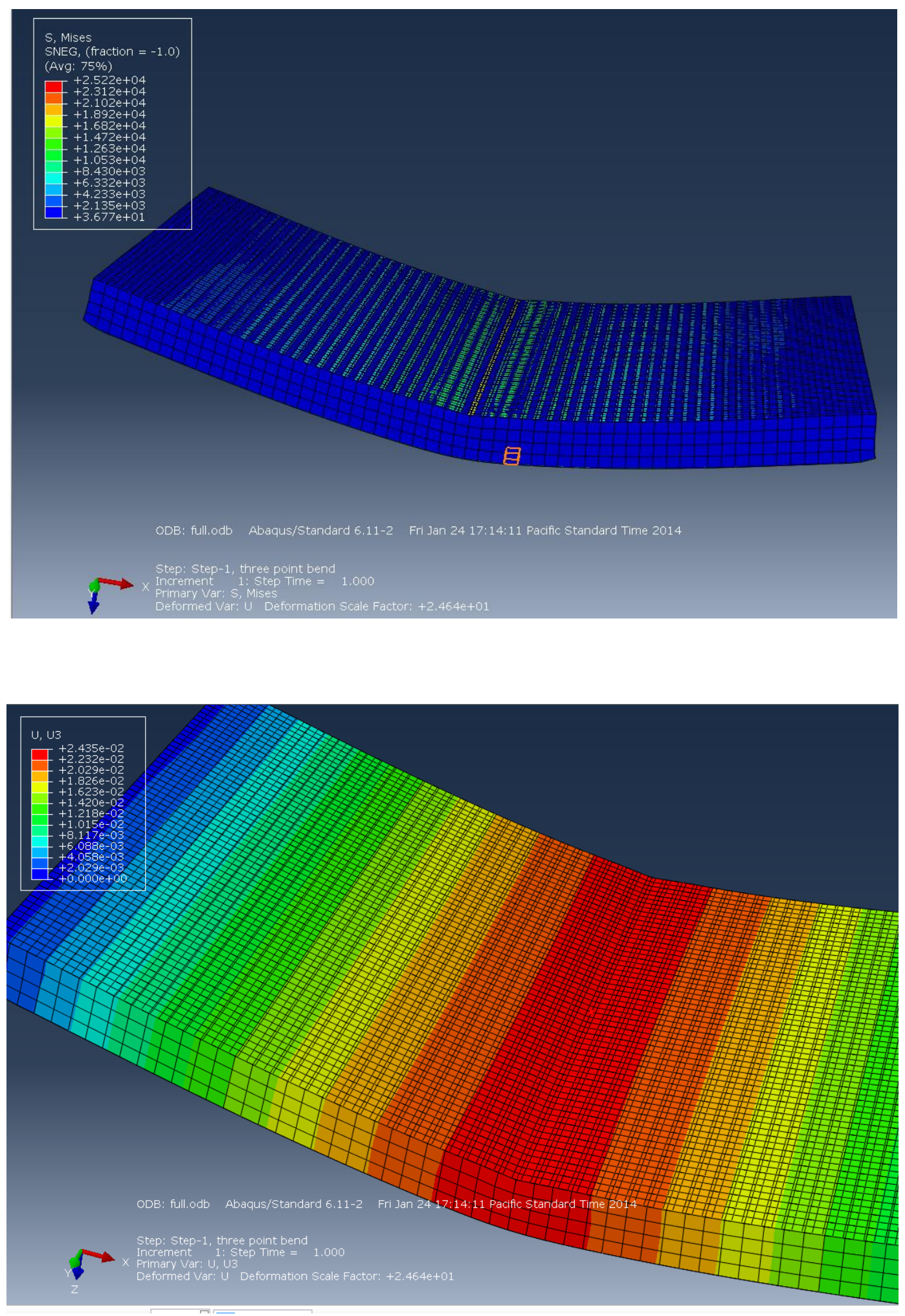


\section{A.8 MATLAB code}

This section includes all MATLAB code used during this thesis.

\section{A.8.1. Classical Beam and Plate Theory for Midspan Deflections of Specimens with Varying Core Ribbon Direction}
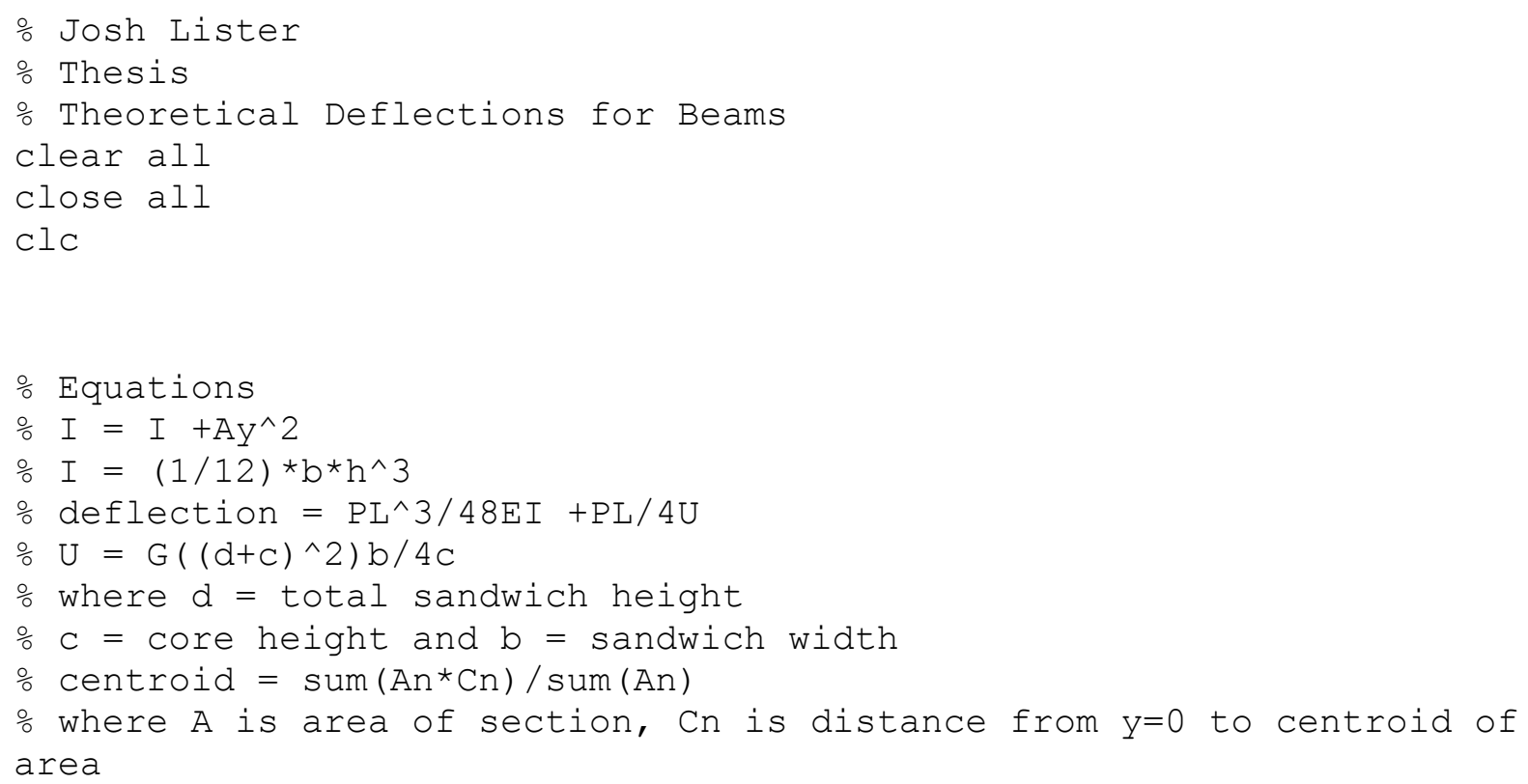

○Constants

$\mathrm{P}=100 ; \quad$ Load (lbf)

$\mathrm{ES}=30 \mathrm{e} 6 ; \quad \quad \frac{\circ}{0} \quad$ Youngs moduli of Steel 6064

VS $=0.297 ; \quad \therefore$ Poissons of steel 6064

$\mathrm{GS}=\mathrm{ES} /(2 *(1+\mathrm{VS})) ; \%$ Core Shear of steel 6064

$\mathrm{Eal}=10 \mathrm{e} 6 ; \quad$ \% youngs moduli of aluminium 1018

val $=0.3 ; \quad \therefore$ Poissons of Aluminium 1018

Gal $=\mathrm{Eal} /(2 *(1+\mathrm{val})) ;$ \% Core Shear of Aluminium 1018

$\mathrm{EC}=12.9 \mathrm{e} 6 ; \quad \quad$ \% Youngs moduli of 6376 A280h carbon fibre

$\mathrm{VC}=0.03 ; \quad \therefore$ Youngs moduli of 6376 A280h carbon fibre

$\mathrm{GC}=\mathrm{EC} /(2 *(1+\mathrm{VC})) ;$ Core Shear of Aluminium 1018

- Beam Dimension

$\mathrm{c}=0.375 ; \quad$ Core Height

$d=0.375+0.048 ; \quad$ \% Sandwich height

tf $=0.024 ; \quad \therefore$ Facesheet height

$\mathrm{b}=1.5 ; \quad \frac{\circ}{0}$ sandwich width

$\mathrm{L}=6 ; \quad \quad \quad$ Sandwich Length

응 Theoretical Complete Steel Beam

Af $=b * t f ; \quad \frac{\circ}{0}$ area of facing

$\mathrm{Ac}=\mathrm{b}{ }^{*} \mathrm{C} ; \quad$ \% area of core

Cf1 $=t f / 2 ; \quad \frac{\circ}{0}$ centroid of bottom facing

Cf2 = tf $+\mathrm{c}+\mathrm{tf} / 2 ; \%$ centroid of top facing 


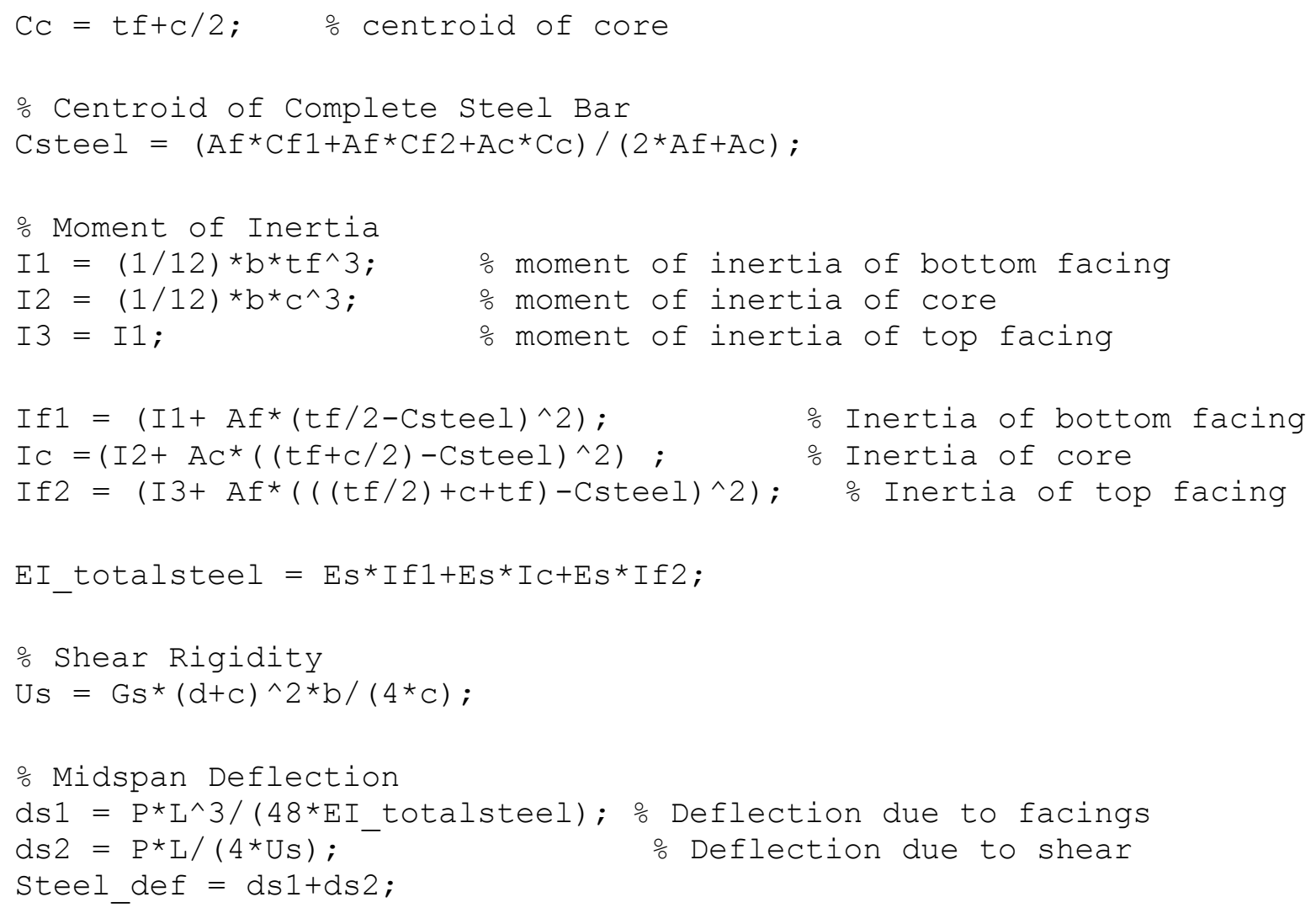




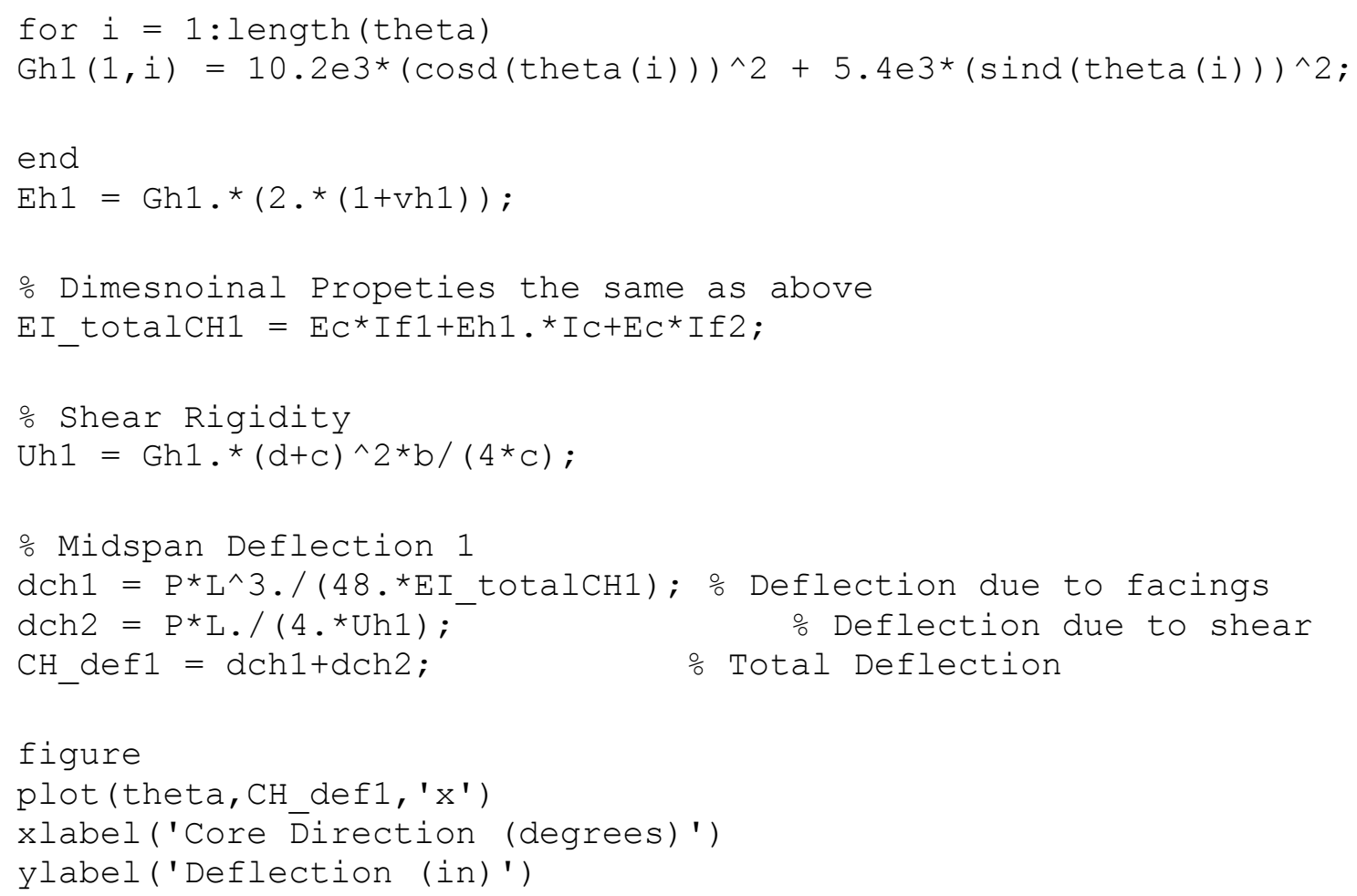

응 Mesh Convergence With Aluminium Face and Steel Core 


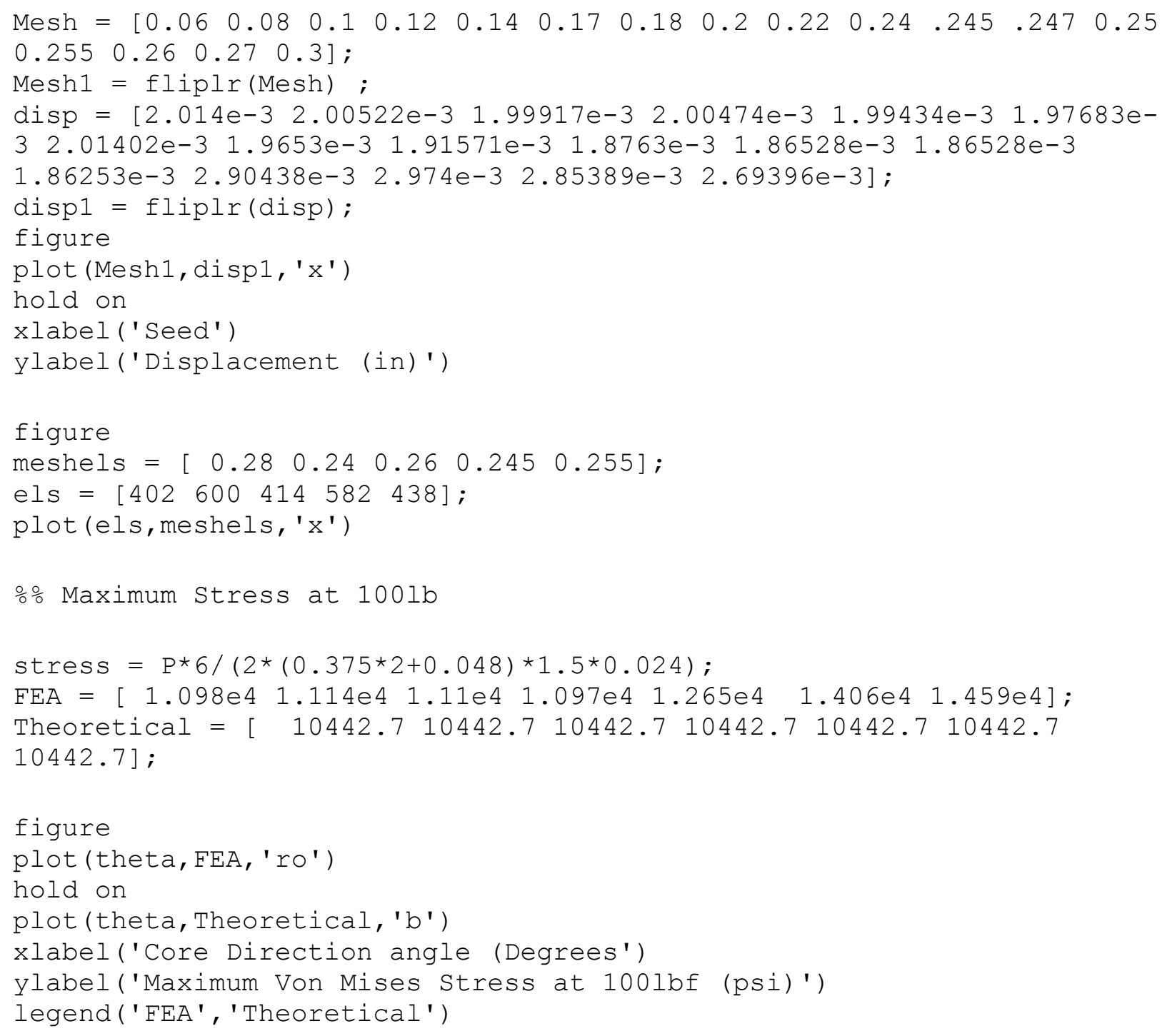

\section{A.8.2. Honeycomb Poisson's Ratio}

$\%$ Honeycomb Core Constants

$\%$

$\%$ Determines experimentally the transvers and longitudinal poissons ratio \% For honeycomb in accordance with ASTM D6790-2

clear all

close all

clc

$\%$ Data

$\% s \#=[c T c L ; d T d L] ;$ inches

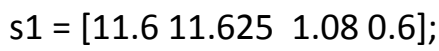




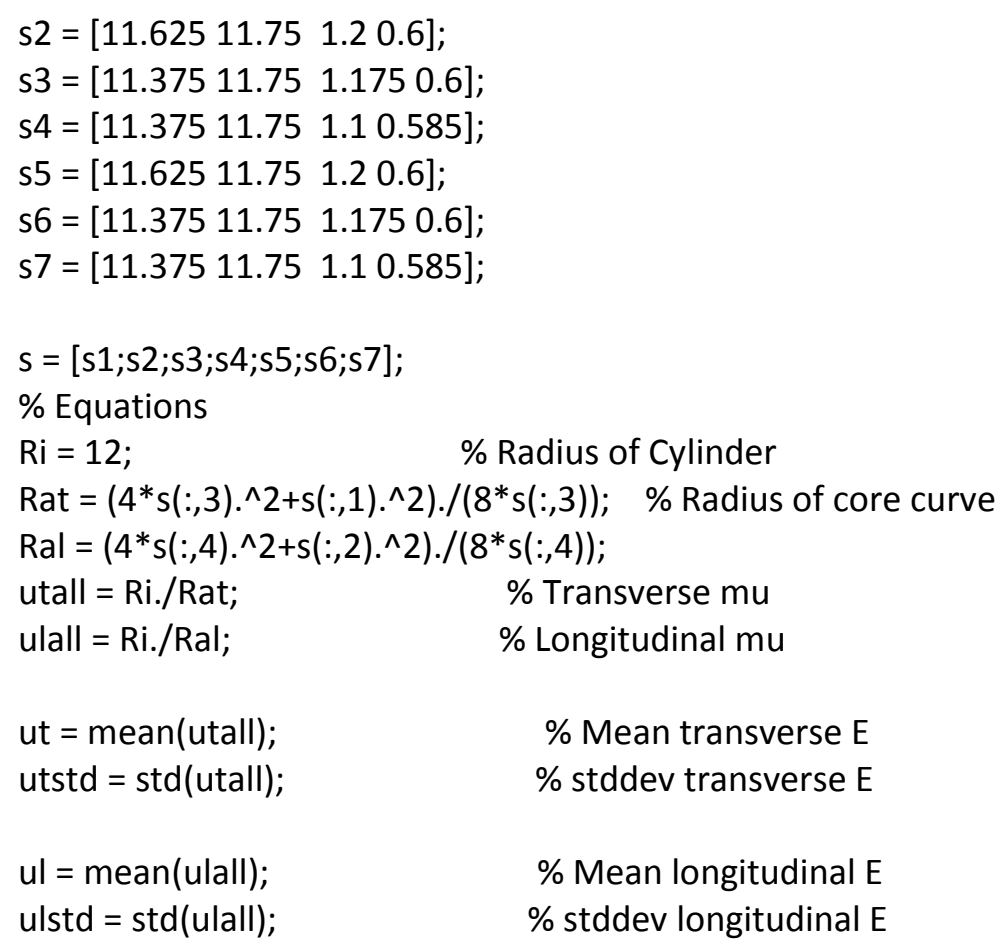

\section{A.8.3. Experimental Density Measurements for Composite Laminates}

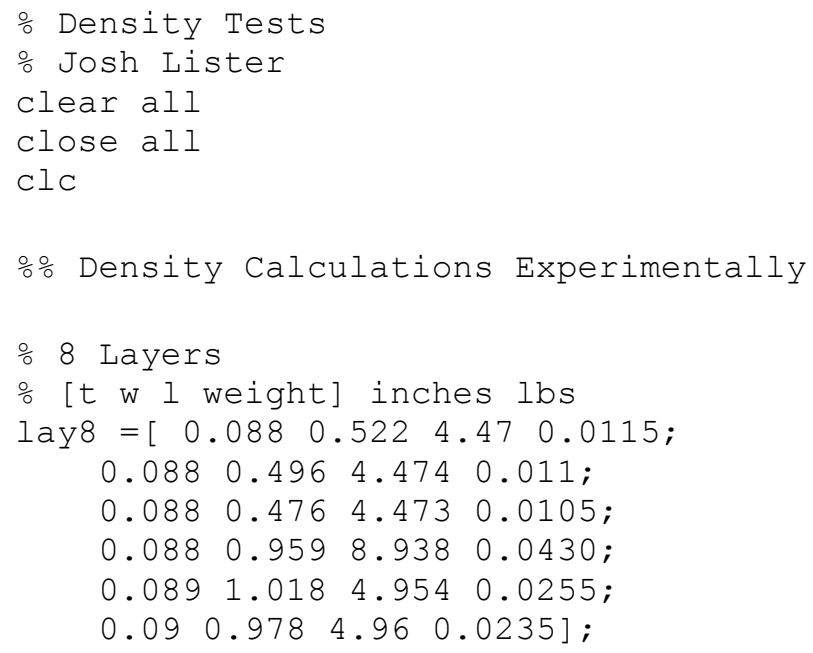




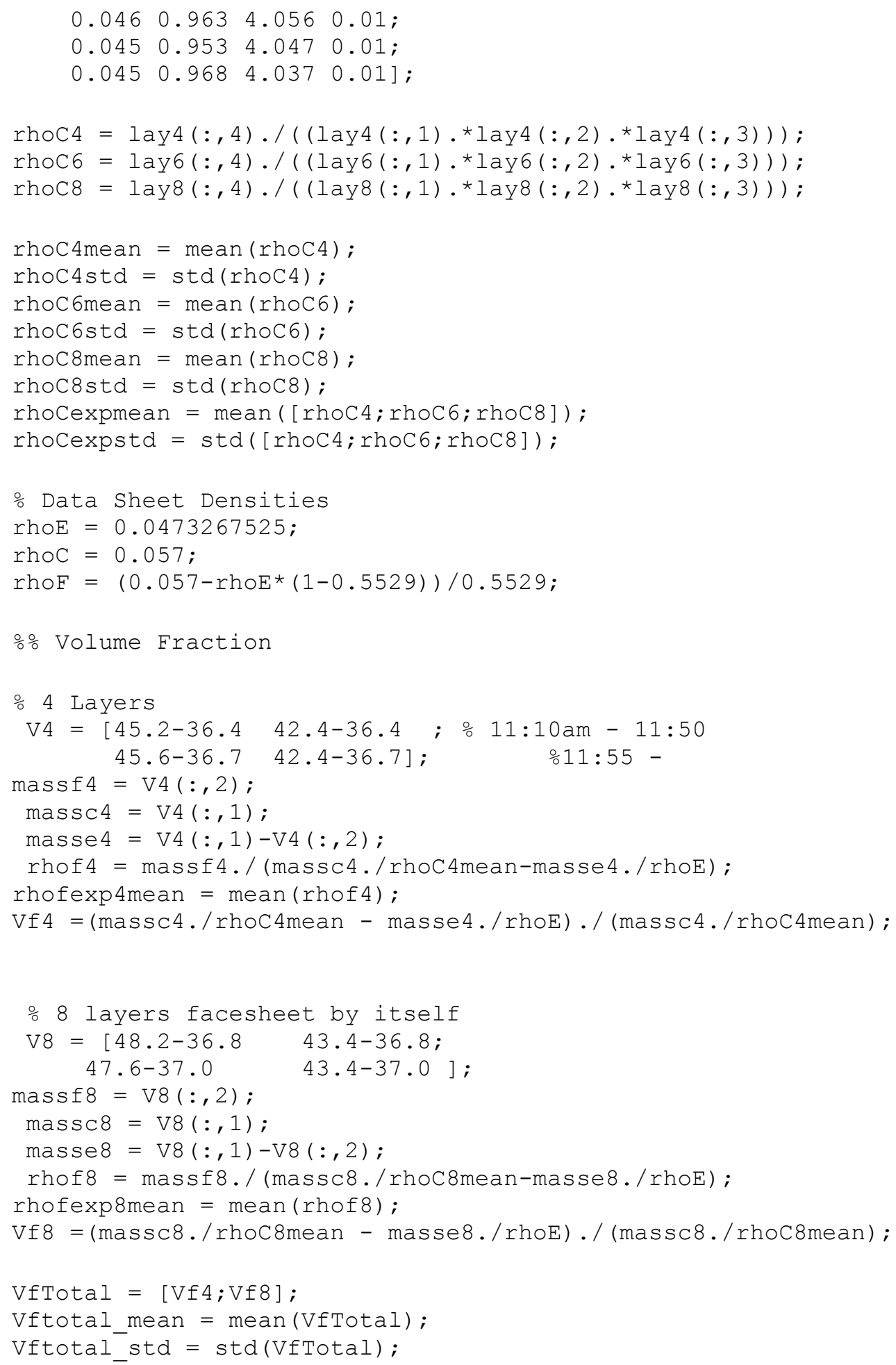

\section{A.8.4. Volume Fraction Calculations}

\% Volume Fraction Tests 


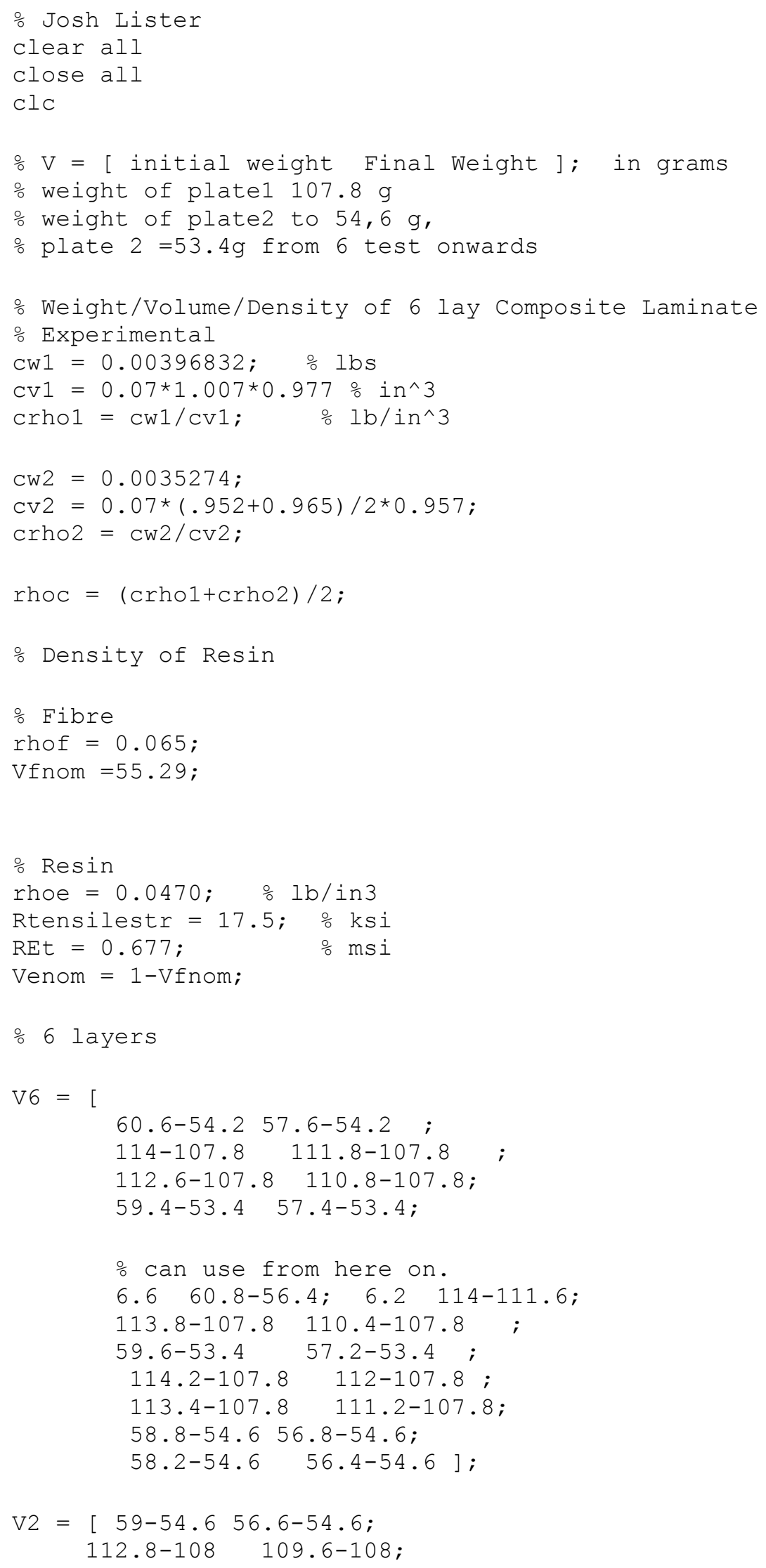


$58.6-53.4 \quad 55.6-53.4 ;$

$57.8-52.8 \quad 0]$;

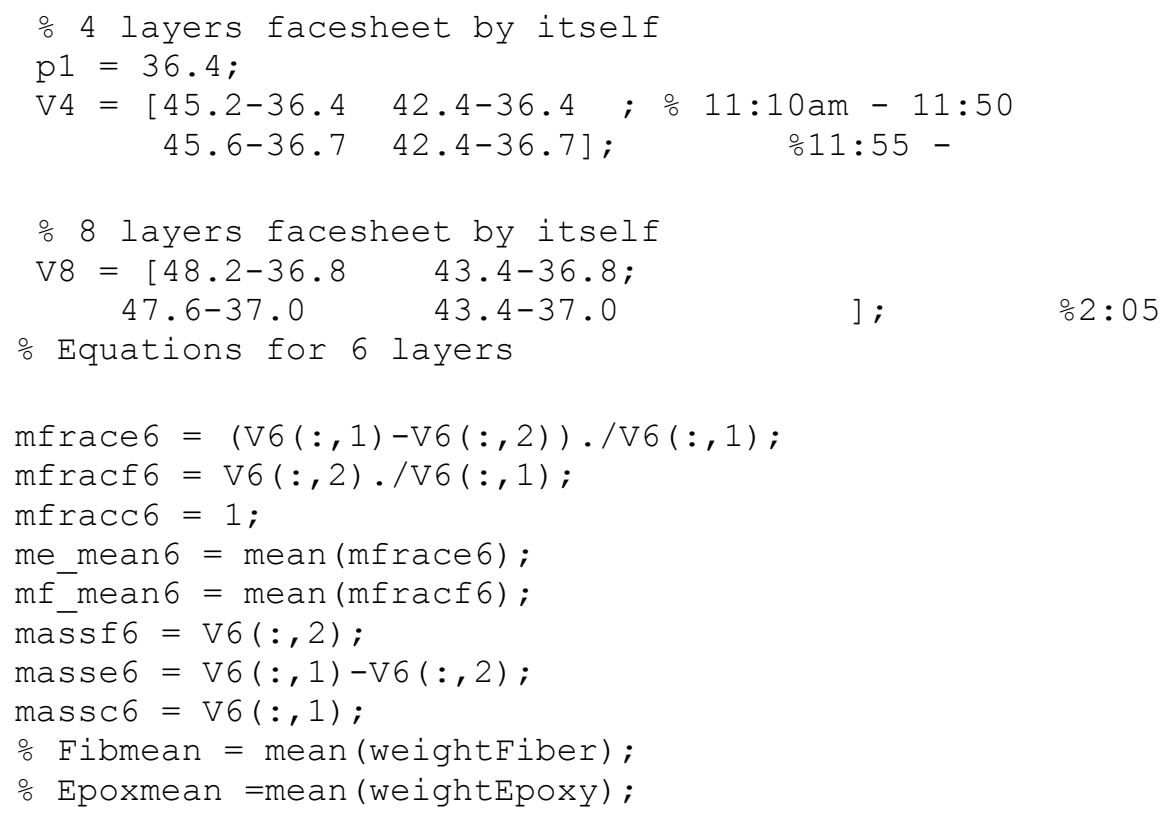




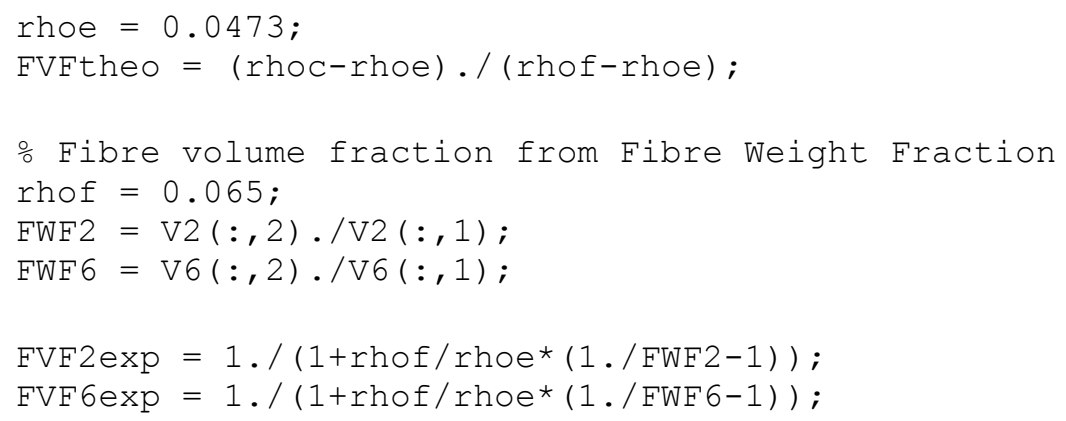

\section{A.8.5. Theoretical Midspan Deflections for FEA Validity}

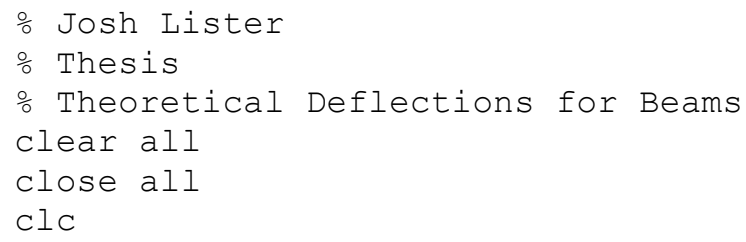




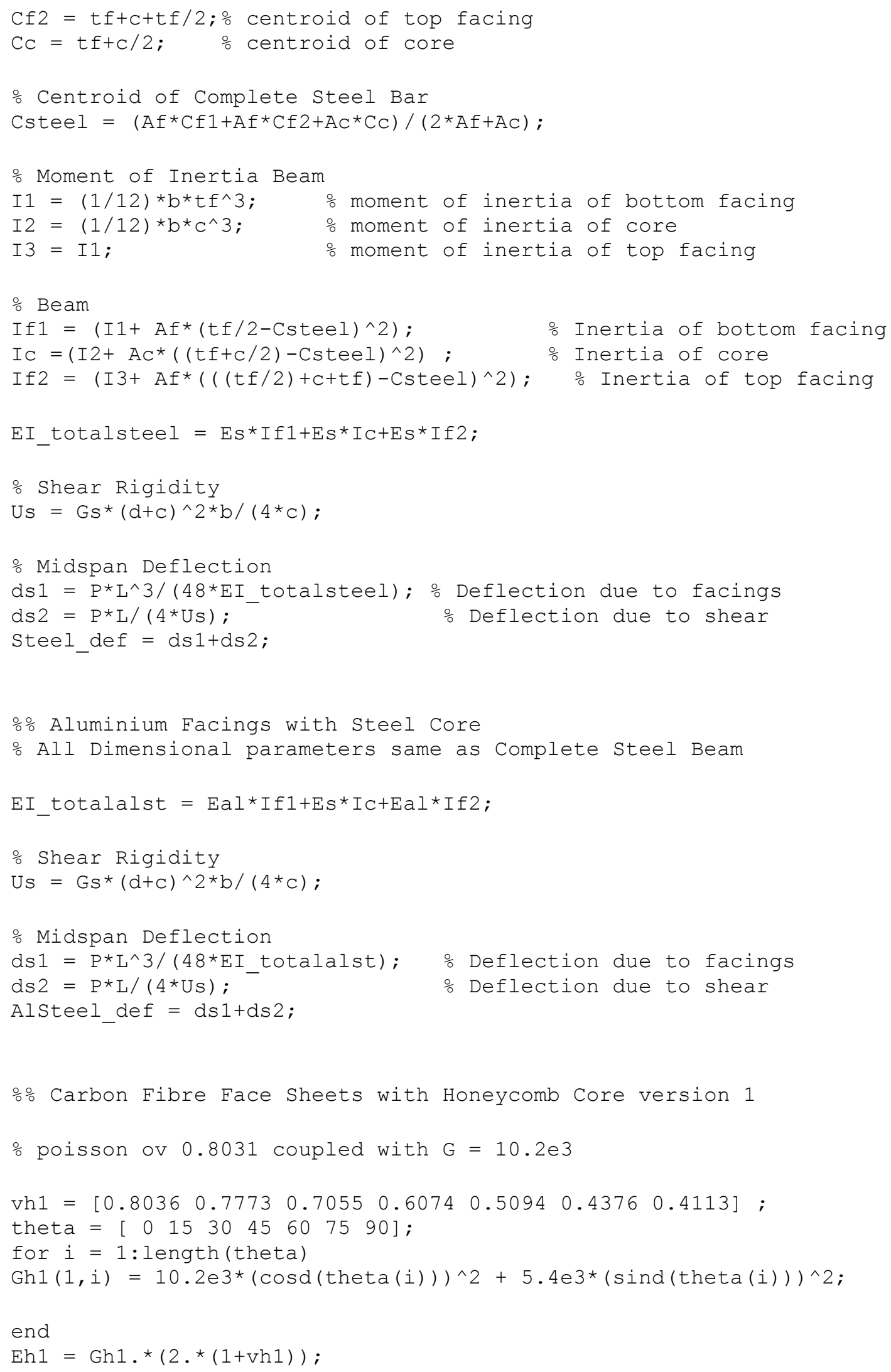


Moment of Inertia Plate

$\mathrm{Ilp}=\left(1 /\left(12 *\left(1-\mathrm{VC}^{\wedge} 2\right)\right)\right) * \mathrm{~b}^{*} \mathrm{t} \mathrm{f}^{\wedge} 3 ; \quad$ moment of inertia of bottom facing

$\mathrm{I} 2 \mathrm{p}=\left(1 . /\left(12 *\left(1-\mathrm{vh} 1 \cdot{ }^{\wedge} 2\right)\right)\right) \cdot{ }^{*} \mathrm{~b}{ }^{*} \mathrm{C} \cdot{ }^{\wedge} 3 ; \quad \frac{\circ}{0}$ moment of inertia of core

$\mathrm{I3P}=\mathrm{Il} ; \quad \frac{\circ}{0}$ moment of inertia of top facing

ㅇ Plate

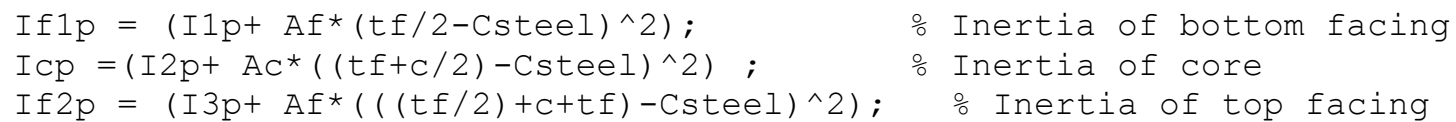

- Dimesnoinal Propeties the same as above

EI totalCH1beam $=E C * I f 1+E h 1 . * I C+E C * I f 2 ;$

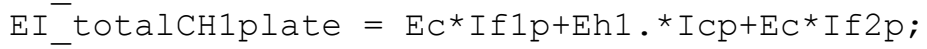

을 Shear Rigidity

Uh1 $=\operatorname{Gh} 1 .{ }^{\star}(\mathrm{d}+\mathrm{c})^{\wedge} 2{ }^{*} \mathrm{~b} /\left(4{ }^{*} \mathrm{c}\right)$;

\% Midspan Deflection beam

dch1 $=\mathrm{P}^{\star} \mathrm{L}^{\wedge} 3 . /\left(48 .{ }^{\star} \mathrm{EI}\right.$ totalCH1beam) ; $\%$ Deflection due to facings

dch2 $=\mathrm{P}^{\star} \mathrm{L} . /\left(4 .{ }^{*} \mathrm{Uh} 1\right) ;-\quad \circ$ Deflection due to shear

$\mathrm{CH} \operatorname{def} 1=\operatorname{dch} 1+\mathrm{dch} 2 ; \quad$ Total Deflection

- Midspan Deflection plate

dch $1 \mathrm{p}=\mathrm{P}^{\star} \mathrm{L}^{\wedge} 3 . /\left(48 .{ }^{*} \mathrm{EI}\right.$ totalCH1plate); $\%$ Deflection due to facings

dch $2 \mathrm{p}=\mathrm{P}^{\star} \mathrm{L} . /\left(4 .{ }^{*} \mathrm{Uh} 1\right) ; \quad$ o Deflection due to shear

CH_defplate1 = dch1p+dch2p; $\quad$ Total Deflection

figure

plot (theta, $\mathrm{CH}$ def1, 'x')

xlabel ('Core Direction (degrees)')

ylabel ('Deflection (in)')

ㅇ Comparison

Experimentald $=\left[\begin{array}{lllllll}0.0337 & 0.0363 & 0.0380 & 0.0453 & 0.0486 & 0.0555 & 0.0558\end{array}\right]$;

expstd $=\left[\begin{array}{lllllll}7.7968 e-4 & 0.0011 & 0.0012 & 0.0013 & 9.1335 e-4 & 0.0022 & 0.0019\end{array}\right]$;

Theoretical beam $=\mathrm{CH}$ defl;

Theoretical plate $=\mathrm{C} \overline{\mathrm{H}}$ defplatel;

$\frac{\circ E A}{\circ}=[3.4 \overline{8} 038 e-0023.58072 e-0023.89588 e-0024.45480 e-0025.23770 e-002$

$6.03650 e-0026.18512 e-002]$;

FEAorthol $=[3.2756 e-23.43119 e-23.87508 e-24.50503 e-25.1571 e-25.64642 e-2$

$5.82776 e-2]$;

FEAortho2 $=[3.37852 e-23.5369 e-23.98261 e-24.61509 e-25.20063 e-25.71674 e-2$

5.91619e-2];

figure

plot (theta, Theoretical_beam, 'ro')

hold on

plot (theta, Theoretical_plate, 'bo')

errorbar (theta, Experimentald, expstd, 'x') 


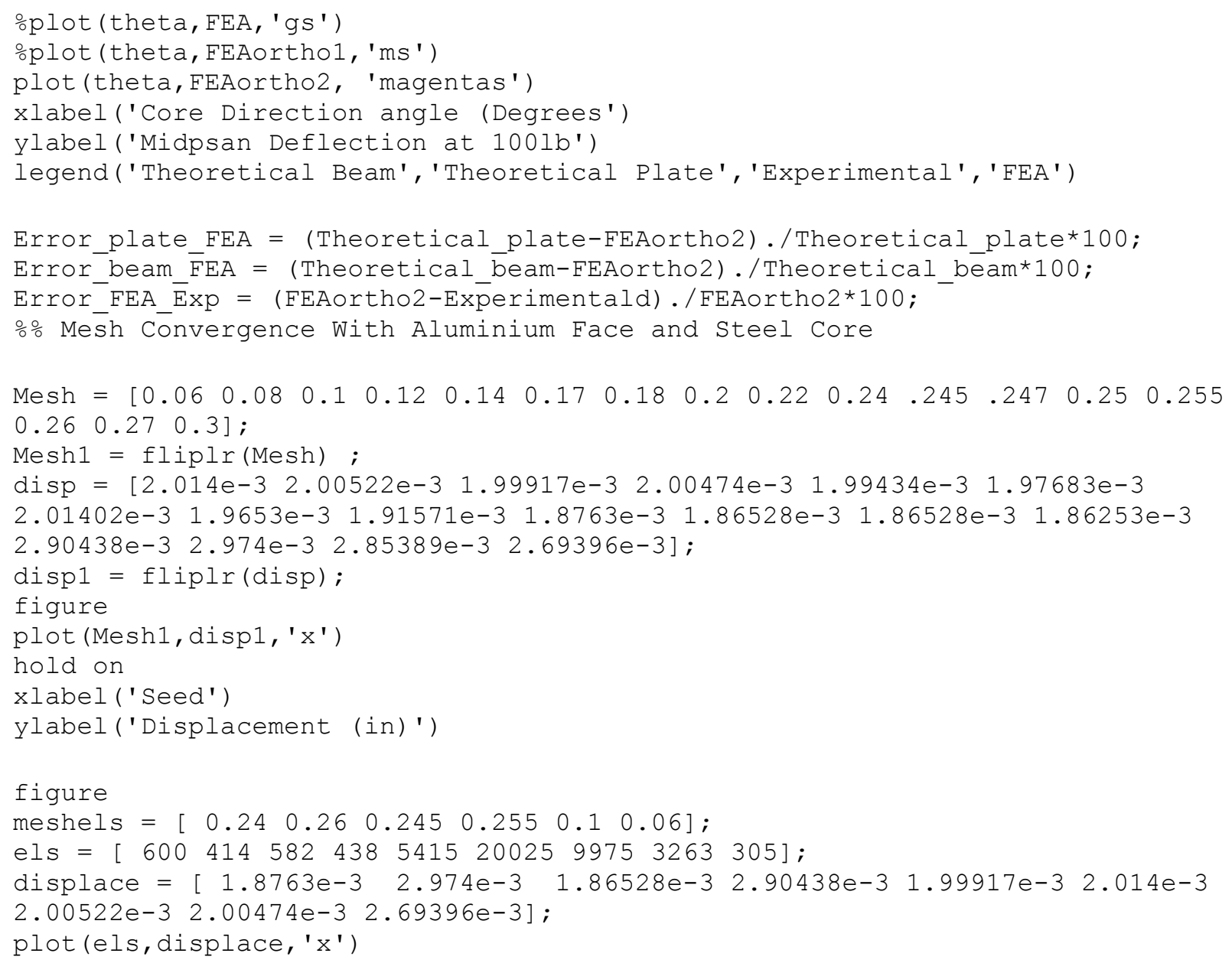

\section{A.8.6. Failure Mode Analysis}

o Static Failure Modes (AERO 500)

clear all; close all; clc

theta $=1$ inspace $(0,90)$;

\% theta $=\left[\begin{array}{lllllll}0 & 15 & 30 & 45 & 60 & 75 & 90\end{array}\right]$;

은 $\operatorname{th}=0$;

응응 Flexural Rigidity

for $i=1:$ length (theta)

Ef $=12.936 \mathrm{e} 6$; $\%$ face Young's modulus (Longitudinal) [psi]

EC_L=3.679e4; 응 core Young's modulus (Longitudinal) [psi]

Ef $W=12.7 e 6$; $\%$ face Young's modulus (Transverse) [psi]

Ec_W=1.5242e4; \% core Young's modulus (Transverse) [psi] 


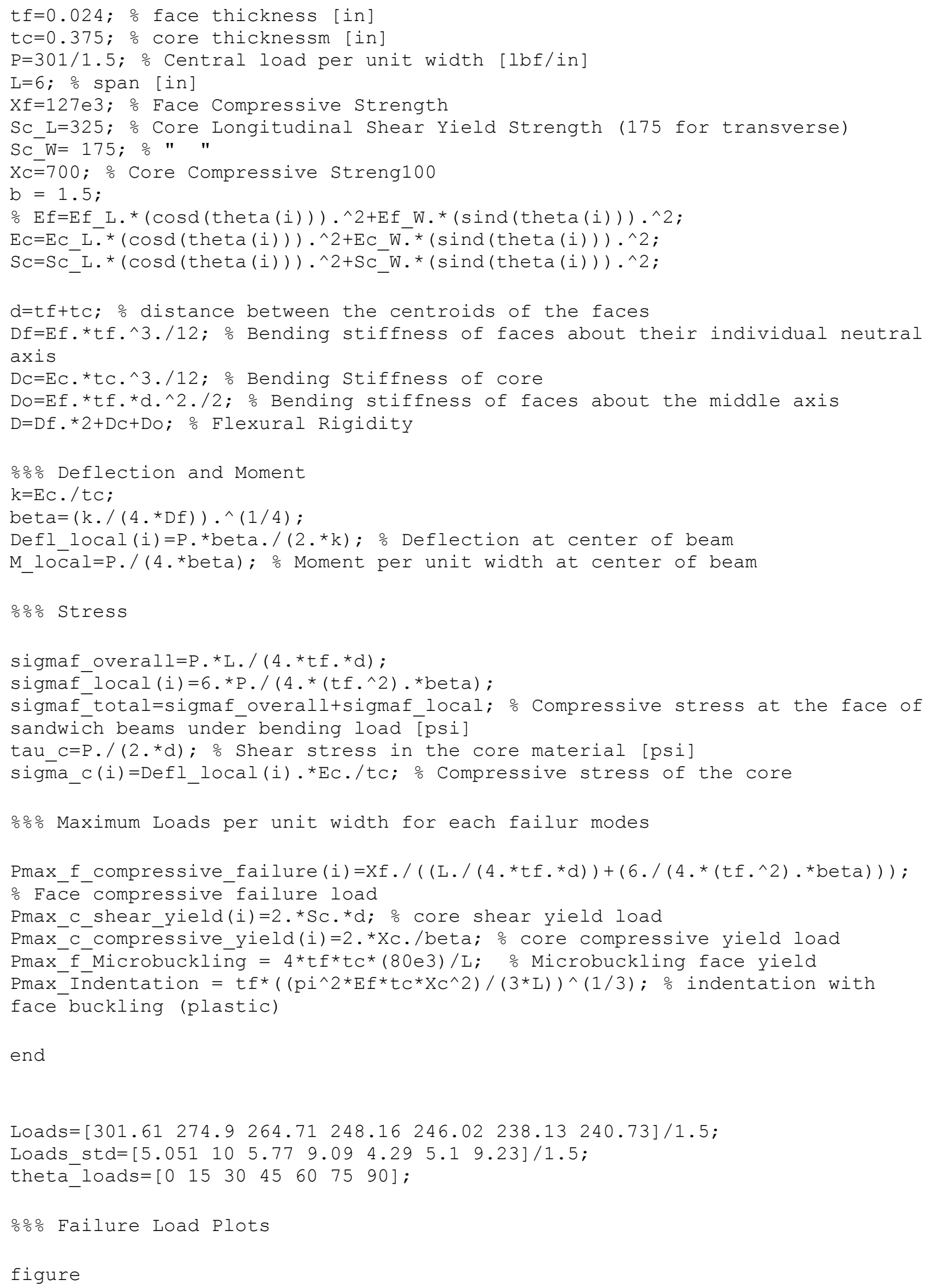




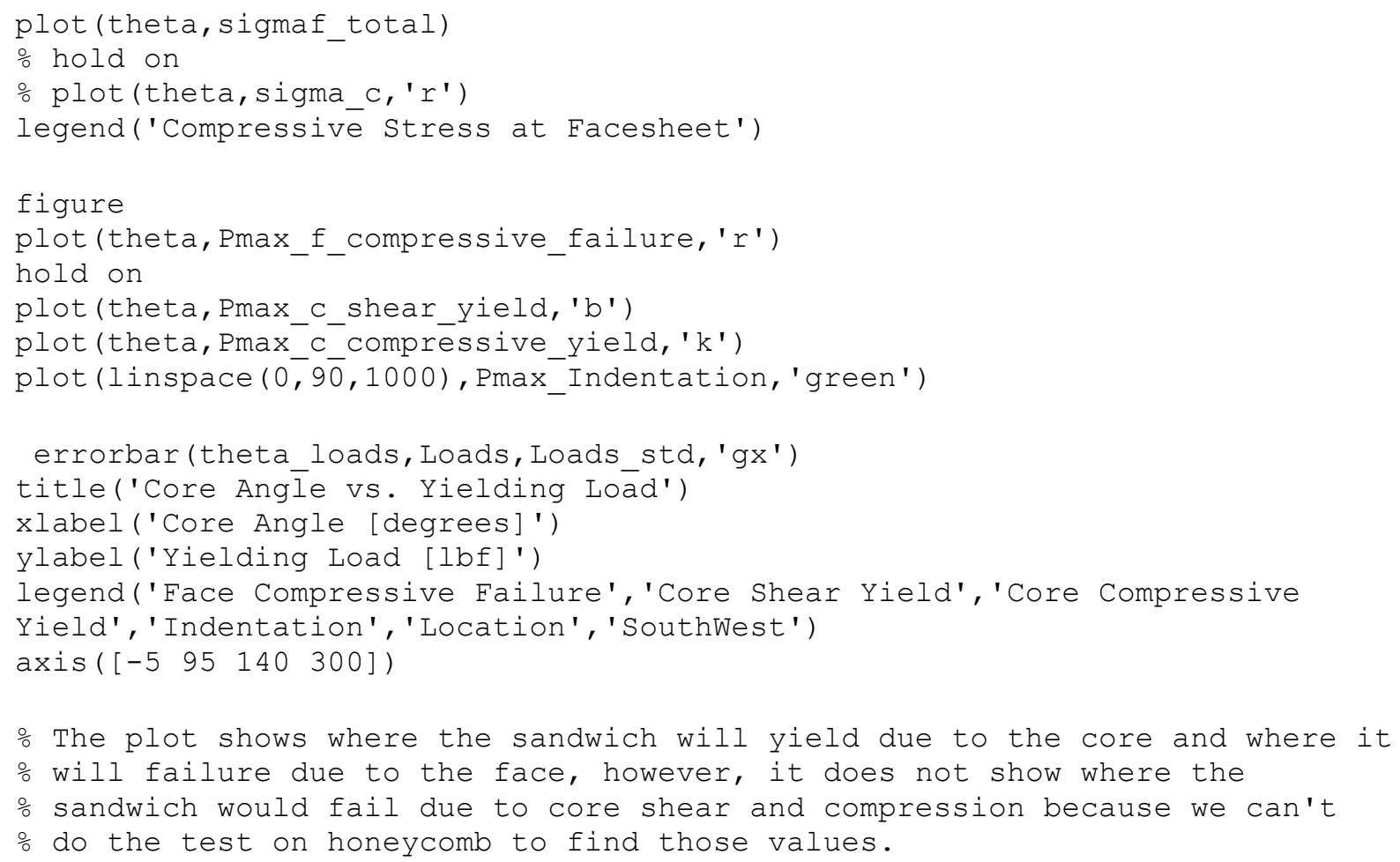




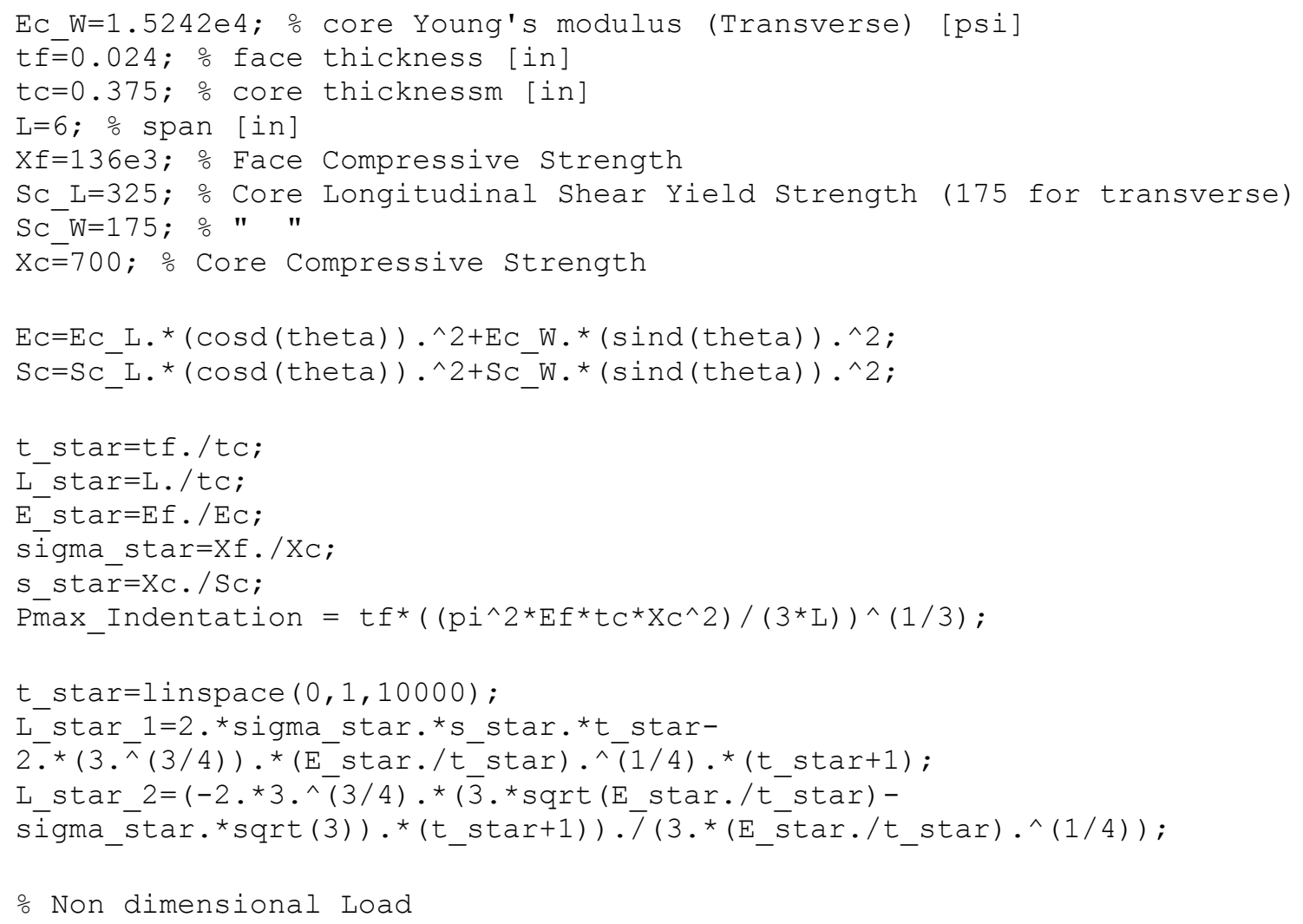




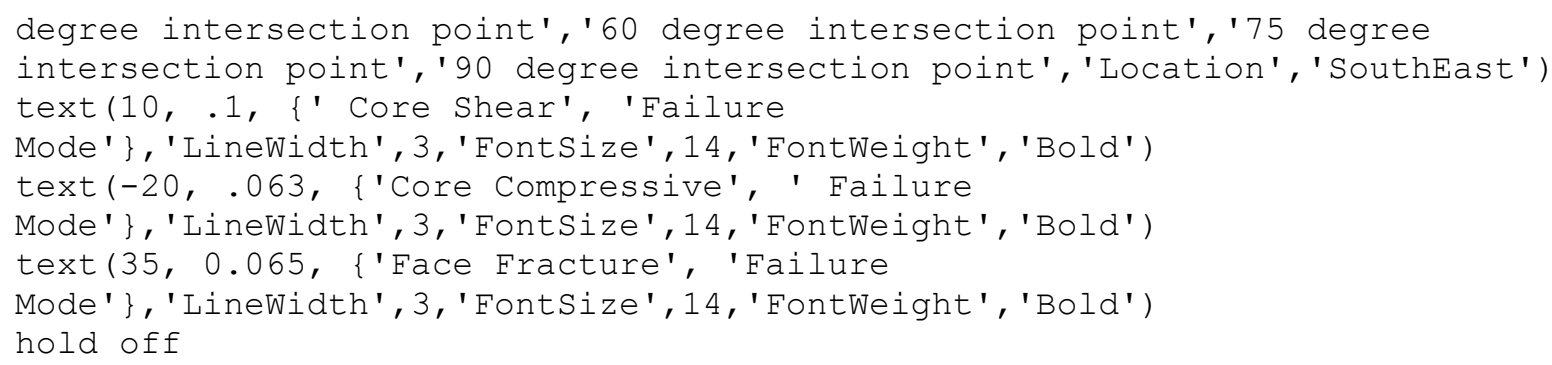




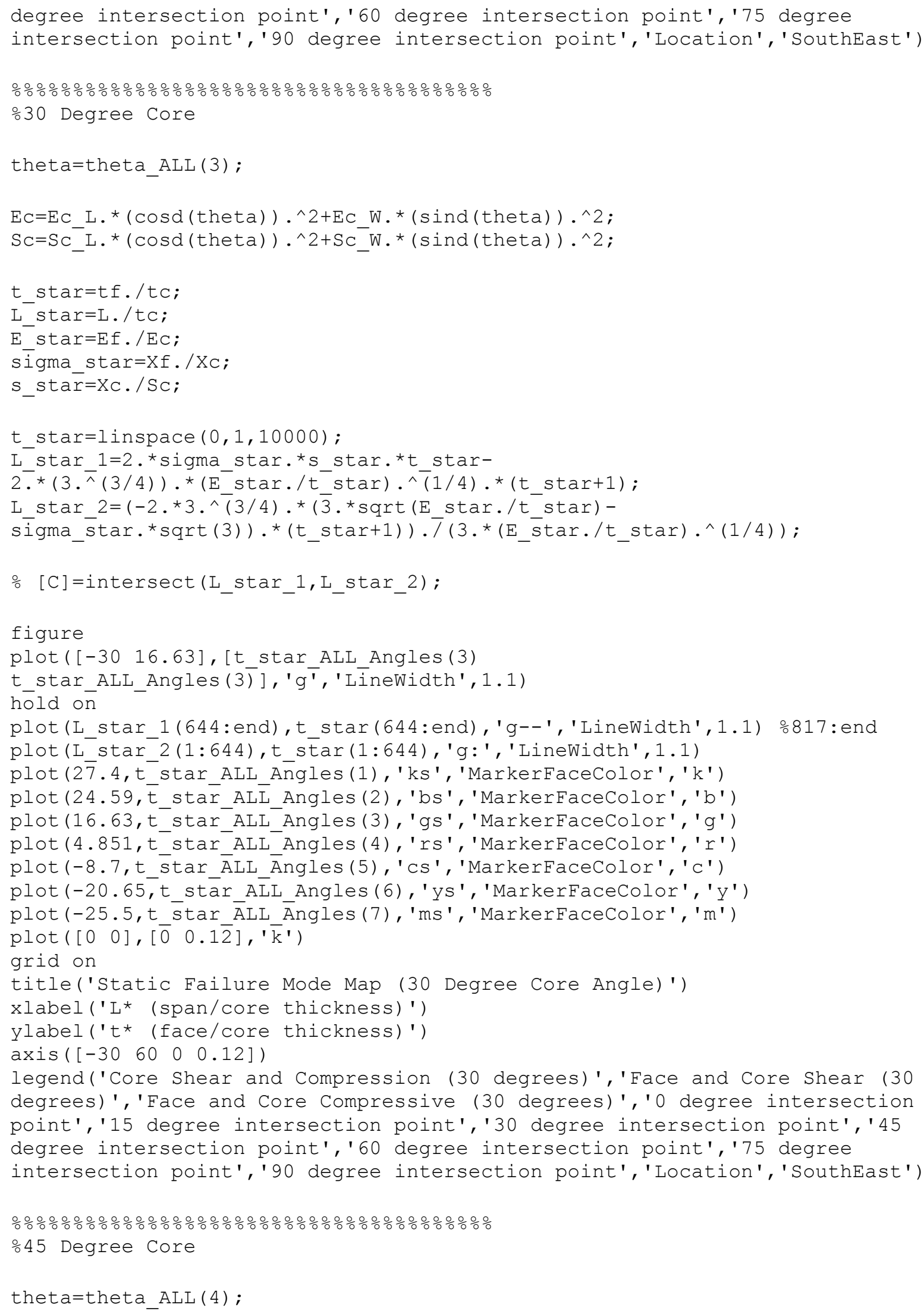




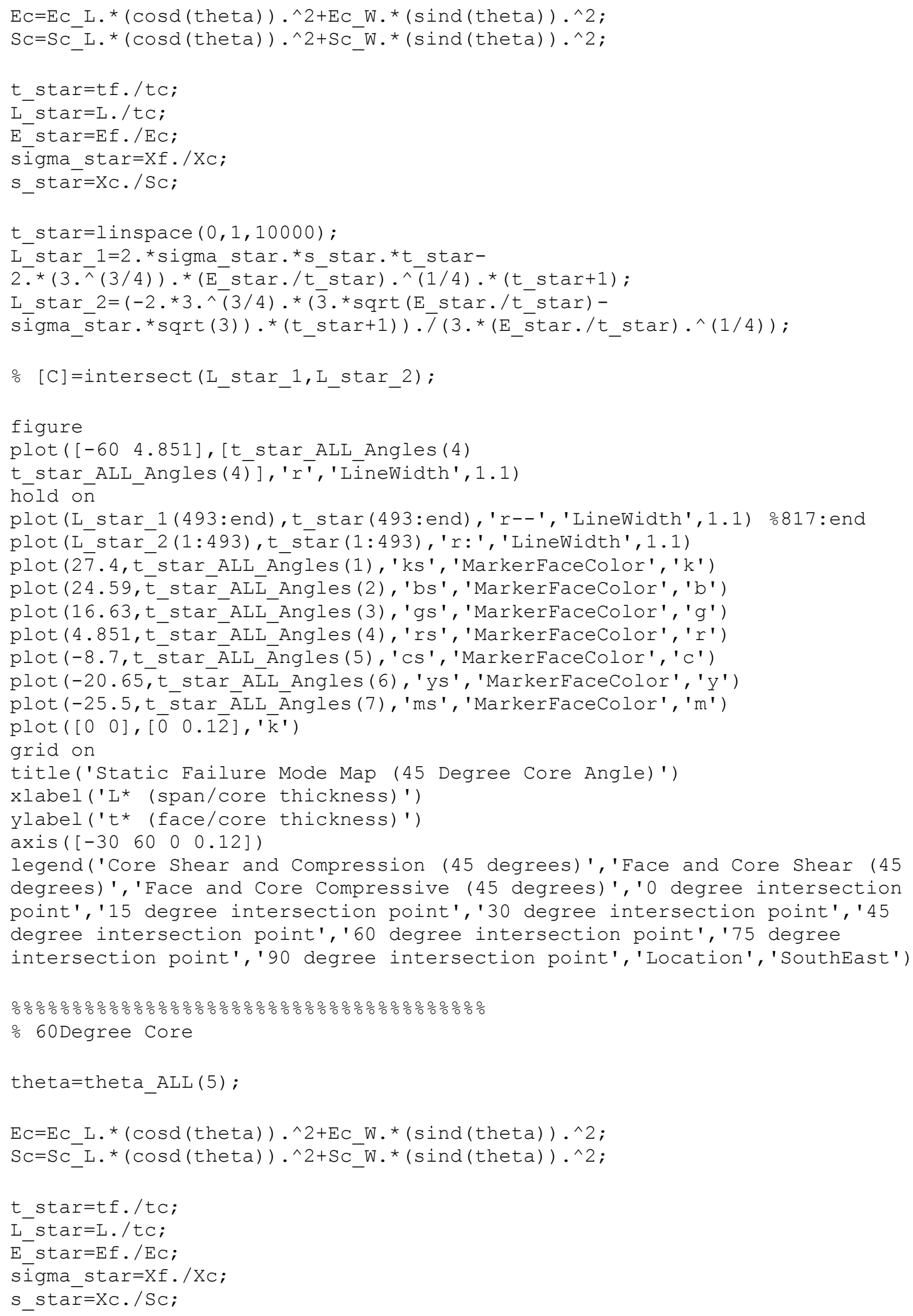




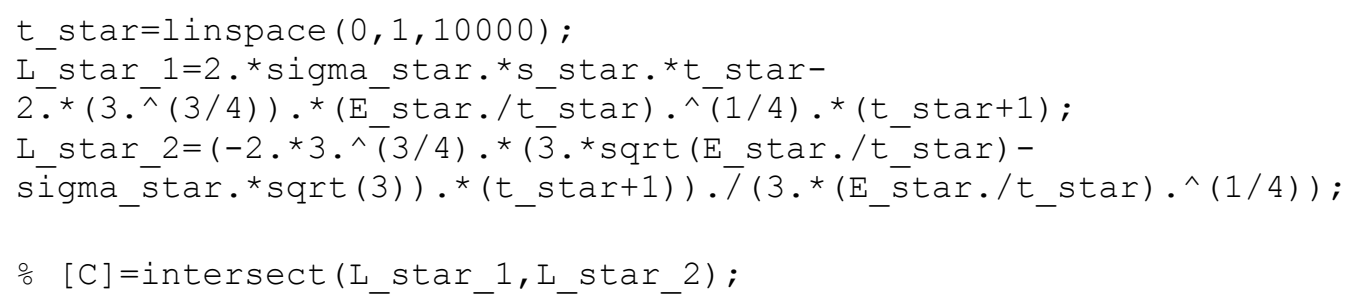


figure

plot ( $[-60-20.65]$, [t star ALL Angles (6)

t star ALL Angles (6) ], 'y', 'Lin̄eWidth', 1.1)

hold on

plot (L star 1 (277:end), t star (277: end), 'y--', 'LineWidth',1.1) 817: end

plot (L_star_2(1:277), t_star(1:277), 'y: ', 'LineWidth',1.1)

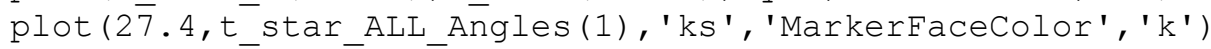

plot (24.59, $\bar{t}$ star_AL__Angles (2),' 'bs', 'MarkerFaceColor', 'b')

plot (16.63, t_star ALLAngles (3), 'gs' ', 'MarkerFaceColor', 'g')

plot (4.851,t_star_ALLAngles (4),'rs', 'MarkerFaceColor','r')

plot (-8.7,t_star_ÄLL_Angles (5), 'Cs', 'MarkerFaceColor', 'C')

plot (-20.65, t_stär ALL Angles (6), 'ys', 'MarkerFaceColor', 'y')

plot (-25.5, t star ĀLL Āngles (7), 'ms', 'MarkerFaceColor' , 'm')

$\operatorname{plot}\left(\left[\begin{array}{ll}0 & 0\end{array}\right],\left[\begin{array}{ll}0 & 0.1 \overline{2}\end{array}\right], ' \overline{\mathrm{k}}^{\prime}\right)$

grid on

title('Static Failure Mode Map (75 Degree Core Angle)')

xlabel ('L* (span/core thickness)')

ylabel ('t* (face/core thickness)')

axis ( [ $\left.\left.\begin{array}{rrrr}-30 & 60 & 0 & 0.12\end{array}\right]\right)$

legend('Core Shear and Compression (75 degrees)','Face and Core Shear (75 degrees)','Face and Core Compressive (75 degrees)','0 degree intersection point','15 degree intersection point','30 degree intersection point','45 degree intersection point','60 degree intersection point','75 degree intersection point','90 degree intersection point', 'Location', 'SouthEast')

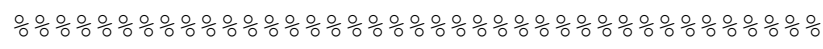

응 Degree Core

theta=theta_ALL (7);

$\mathrm{EC}=\mathrm{EC} \_$L.* $(\operatorname{cosd}($ theta $)) \cdot{ }^{\wedge} 2+\mathrm{EC} W \mathrm{~W} \cdot{ }^{*}(\operatorname{sind}($ theta $)) \cdot{ }^{\wedge} 2$;

$\mathrm{SC}=\mathrm{Sc} \_$L. ${ }^{*}(\operatorname{cosd}($ theta $)) \cdot{ }^{\wedge} 2+\mathrm{SC}_{-} W \cdot{ }^{*}(\operatorname{sind}($ theta $)) \cdot{ }^{\wedge} 2$;

t_star=tf./tc;

L_star=L./tc;

E_star=Ef./EC;

sigma_star=Xf./Xc;

s_star $=\mathrm{Xc} . / \mathrm{Sc}$;

$t$ star=linspace $(0,1,10000)$;

$\mathrm{L}$ star $1=2 .{ }^{*}$ sigma star. ${ }^{*} \mathrm{~s}$ star. ${ }^{*} t$ star -

$2 \cdot{ }^{*}\left(3 \cdot{ }^{\wedge}(3 / 4)\right) \cdot{ }^{*}\left(\mathrm{E}^{-} \operatorname{star} \cdot / t_{\text {star }}\right) \cdot{ }^{\wedge} \overline{(}(1 / 4) \cdot{ }^{*}\left(t \_s t a r+1\right) ;$

L_star_2 $=\left(-2 \cdot * 3 \cdot{ }^{\wedge}(3 / 4) \cdot *\left(\overline{3} \cdot{ }^{*} \operatorname{sqrt}\left(\mathrm{E} \_\right.\right.\right.$star. $/ \mathrm{t}_{-}$star $)-$

sigma_star.*sqrt (3)).* (t_star+1)).T(3.*(E_star./t_star).^(1/4));

$\div \quad[\mathrm{C}]=$ intersect $\left(\mathrm{L}_{-}\right.$star_1, L_star_2) ;

figure

plot ( [-60 -25.5], [t star ALL Angles (7)

t_star_ALL_Angles (7) ], 'm' ', 'LineWidth',1.1)

hold on

plot (L_star_1 (249:end), t_star(249:end), 'm--', 'LineWidth',1.1) 817: end

plot(L_star_2(1:249), t_star(1:249), 'm:', 'LineWidth',1.1)

plot (27.4,t_star_ALL_Angles (1),' ks' ', 'MarkerFaceColor', 'k') 


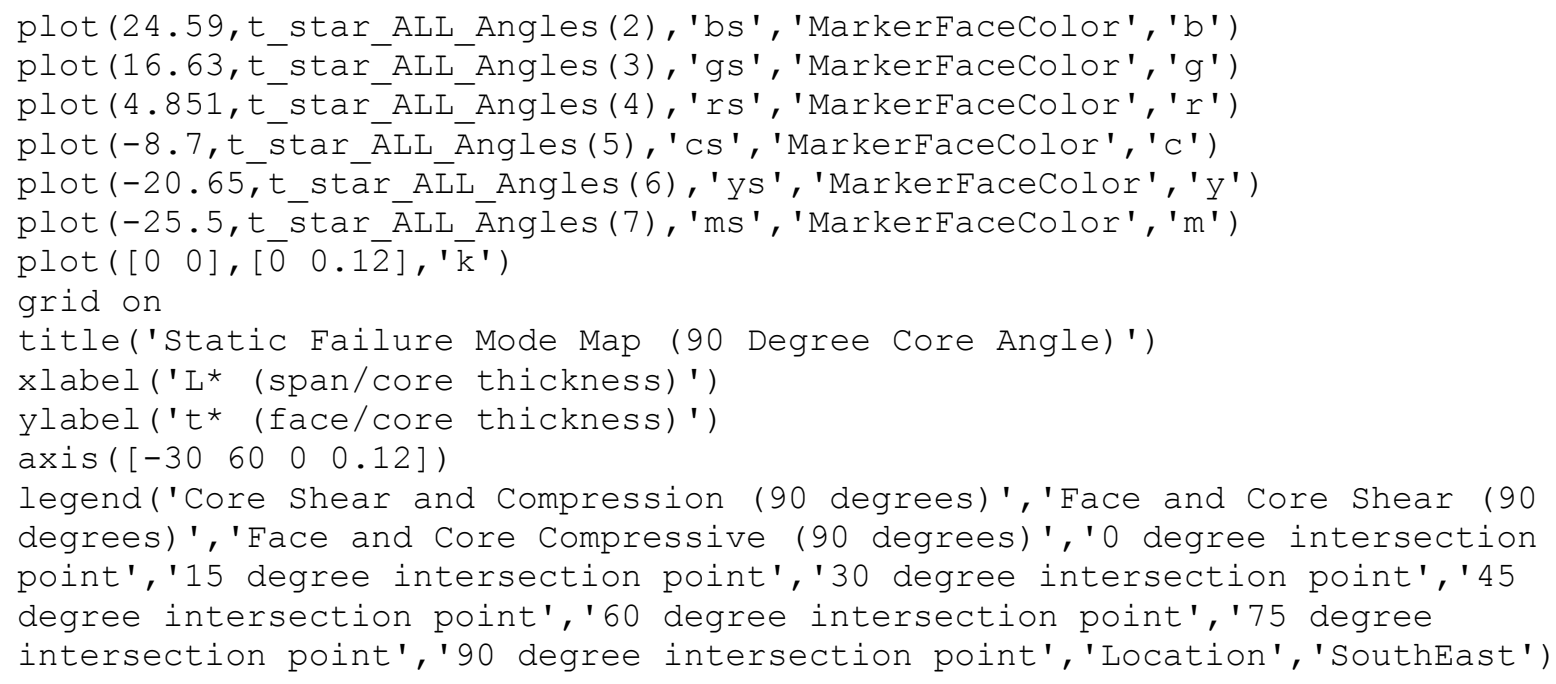

\section{A.8.7. Master Code for all varying face thickness matlab files.}

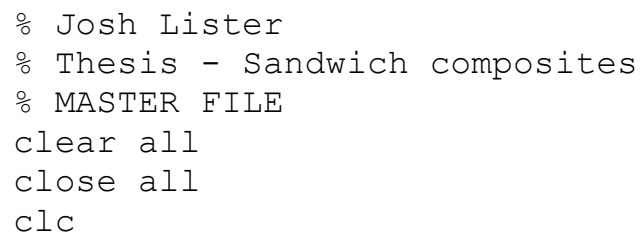


while $i==6|| 7|| \begin{array}{llll}1 & = & 19\end{array}$

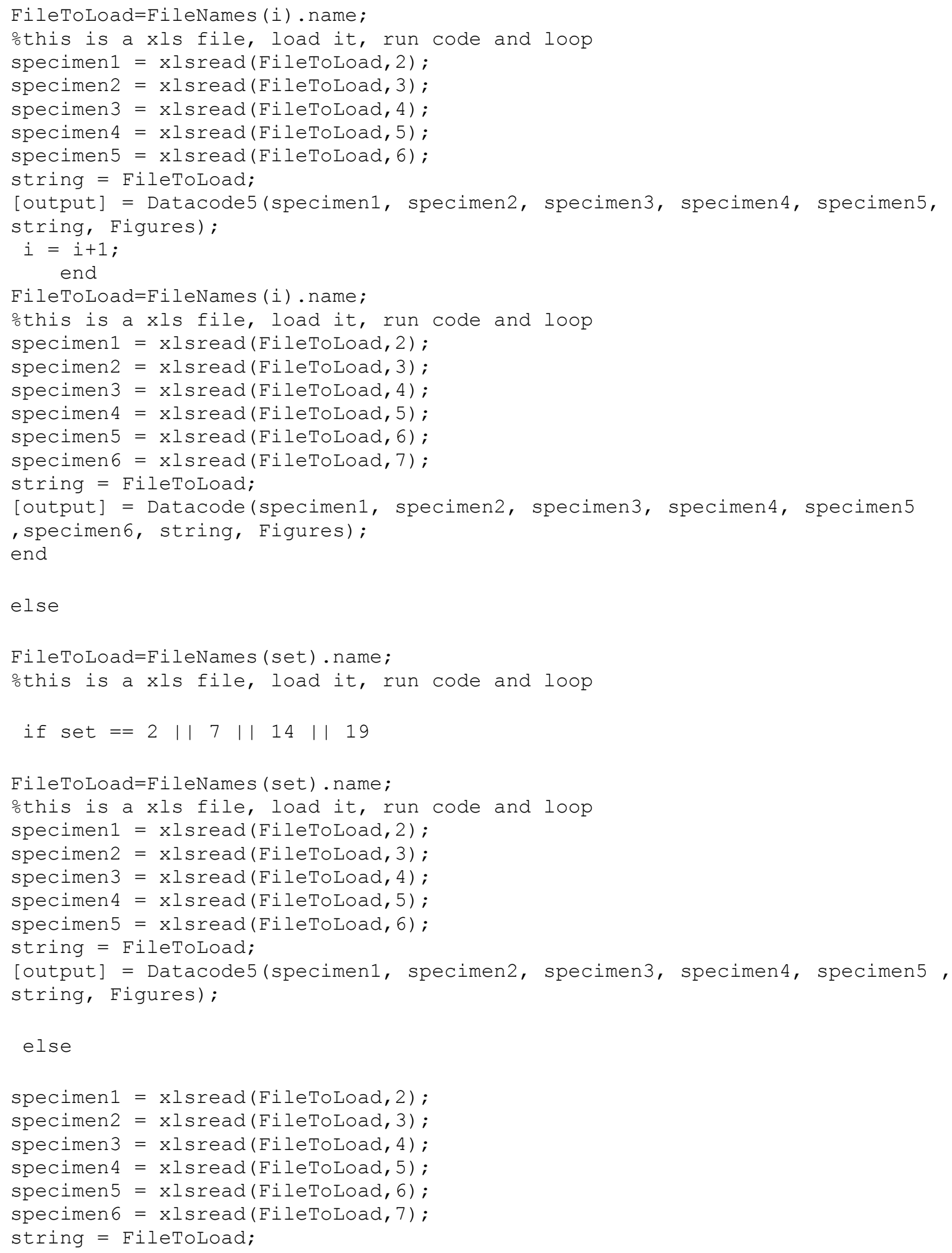


[output] = Datacode(specimen1, specimen2, specimen3, specimen4, specimen5 , specimen6, string, Figures, set)

\% output $=[$ P Ex D U G F sigmalult sigma2ult Exten200avg Pstd Exstd Ex200std Kstd]

end

end

\section{A.8.8. Data Manipulation Code for Varying Face Sheet Thickness Specimens}

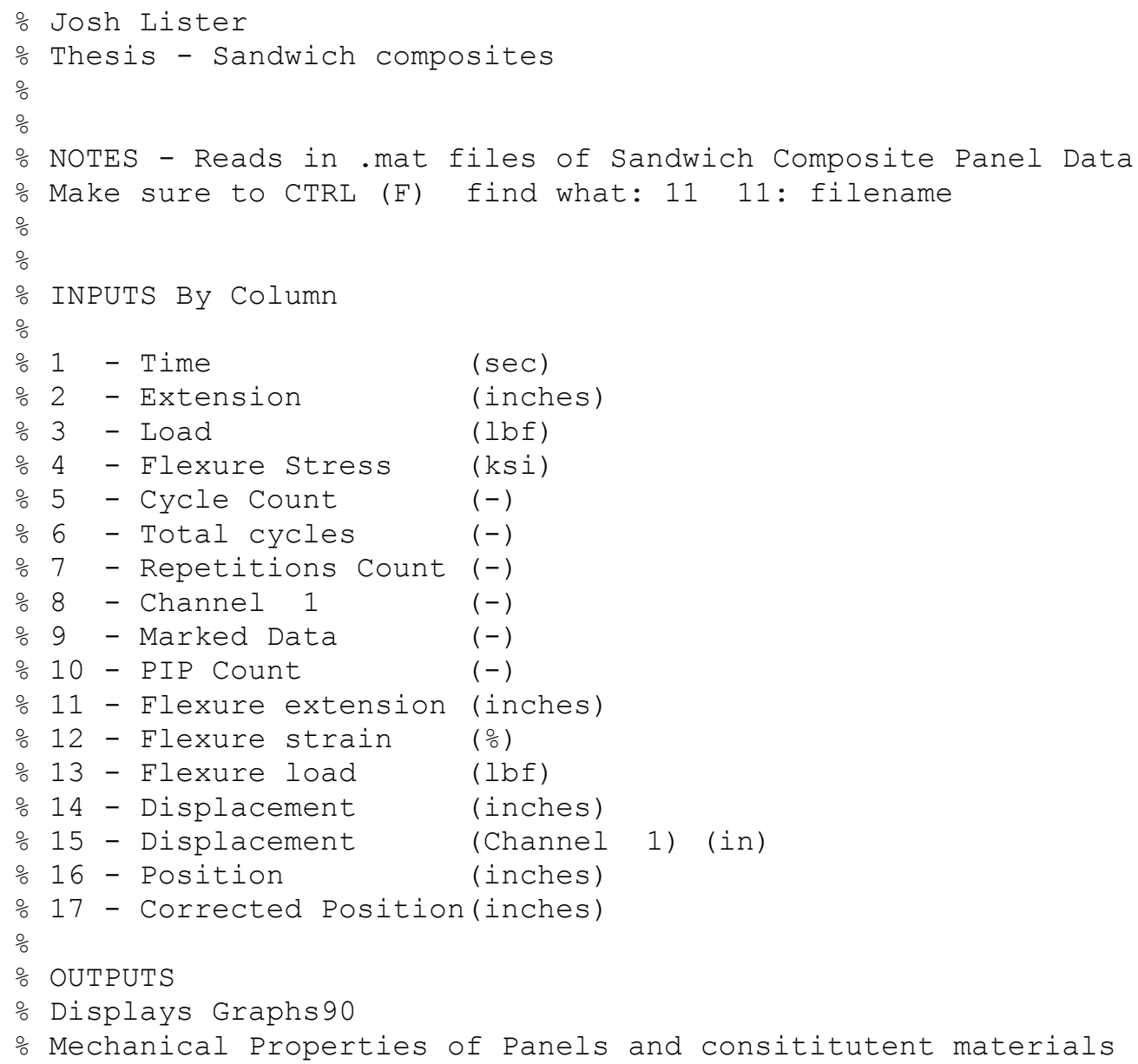




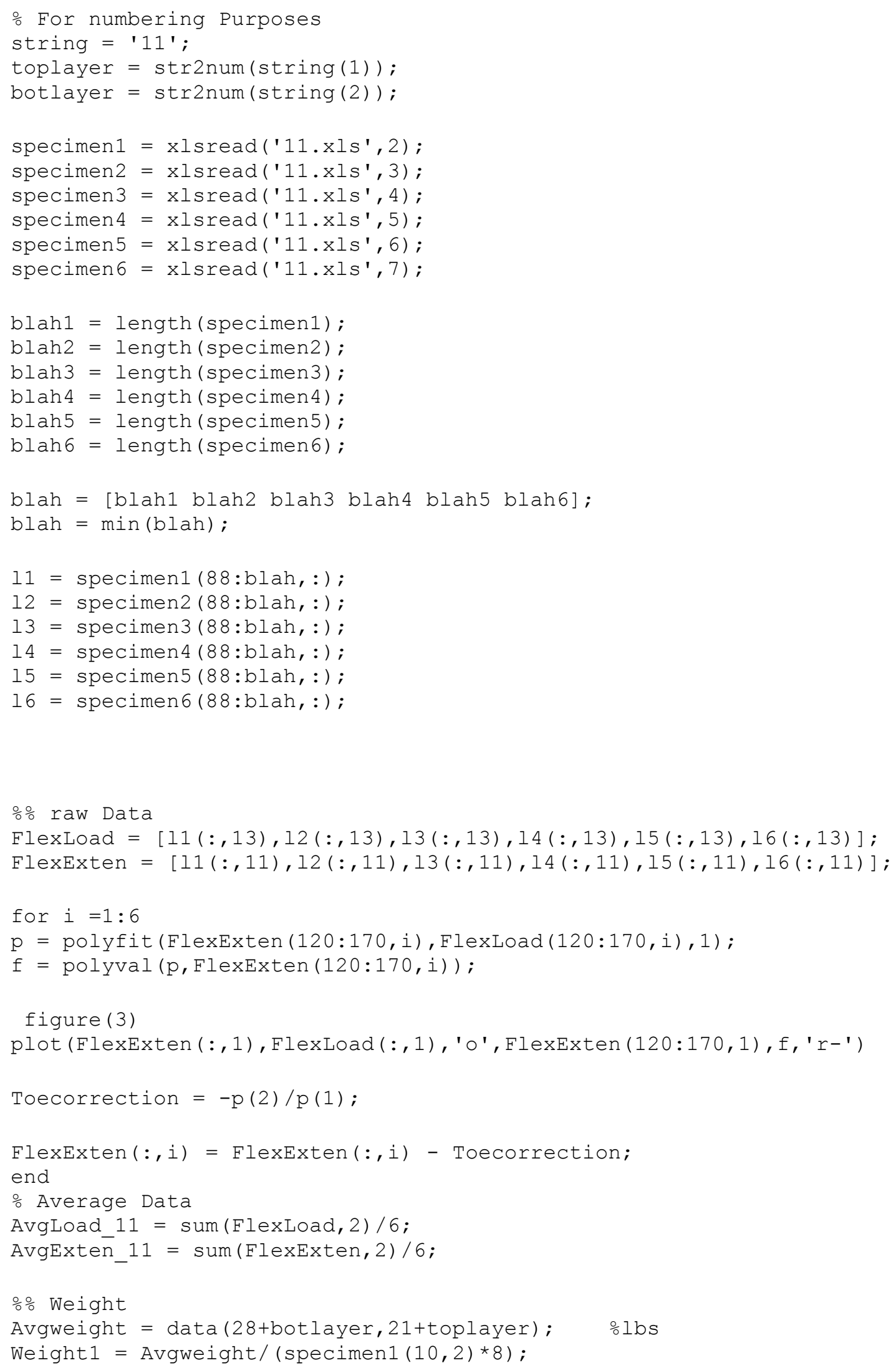




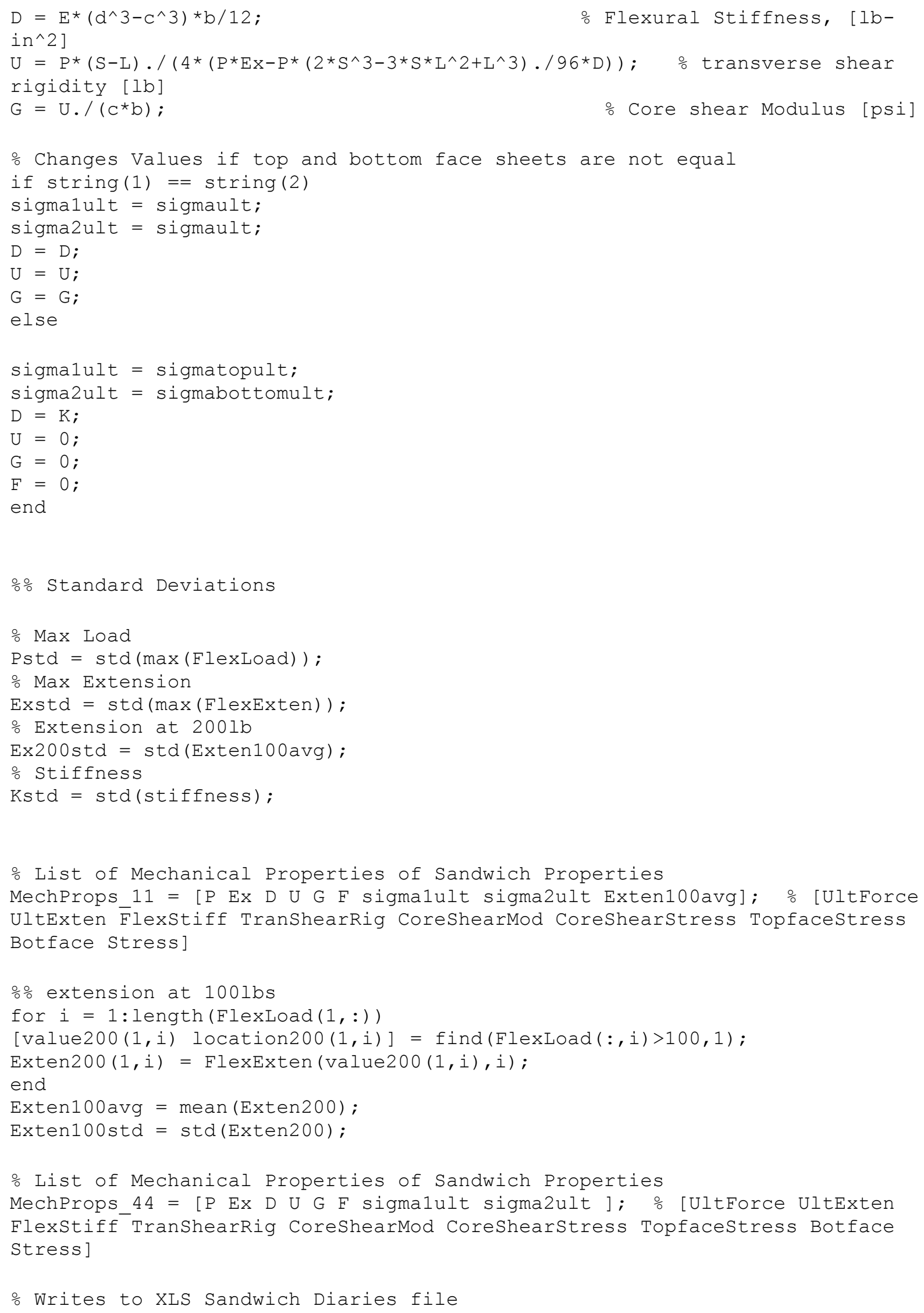




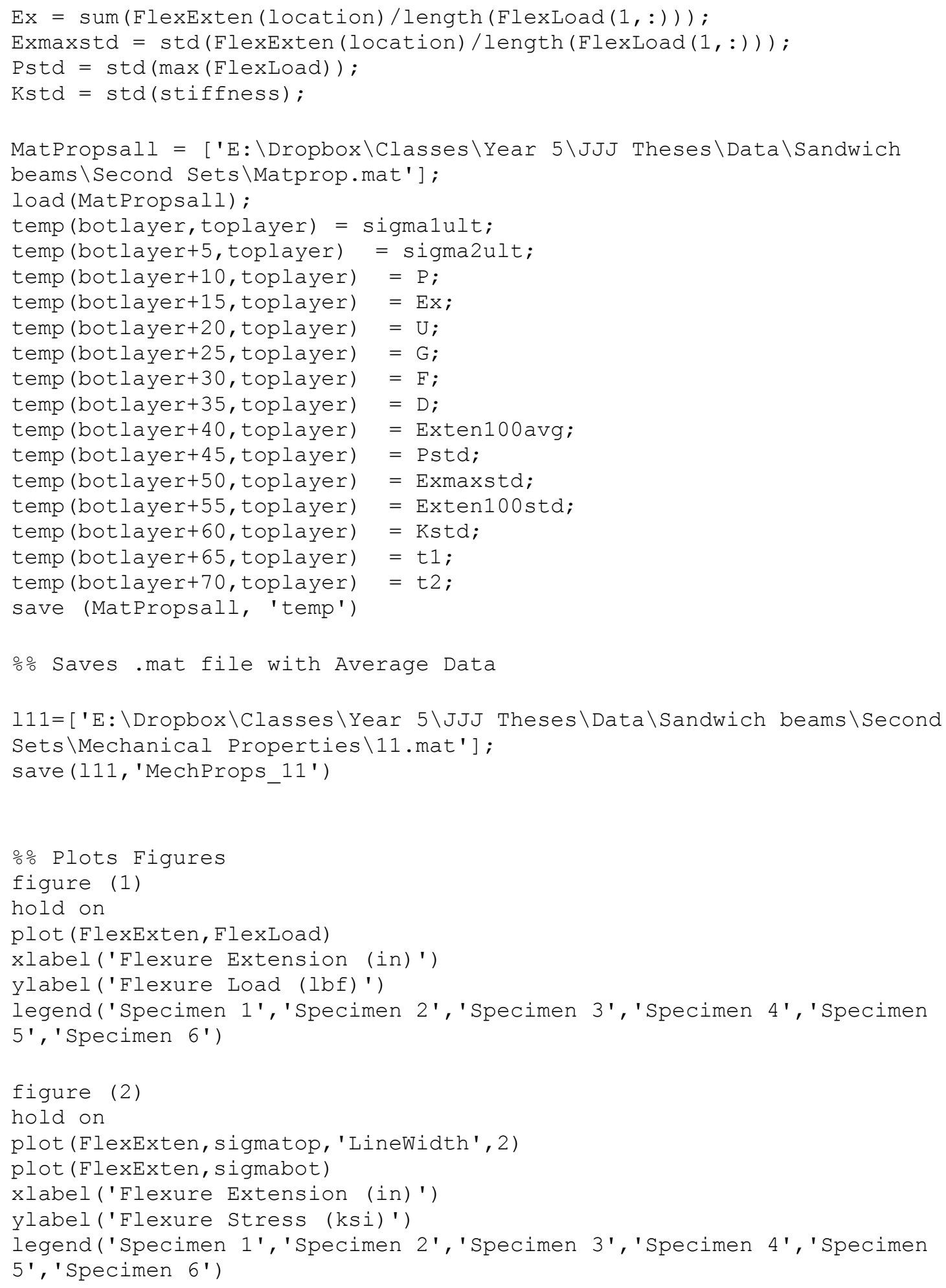




\section{A.8.9. Plots Experimental Graphs for Varying Face Sheet Thickness's}

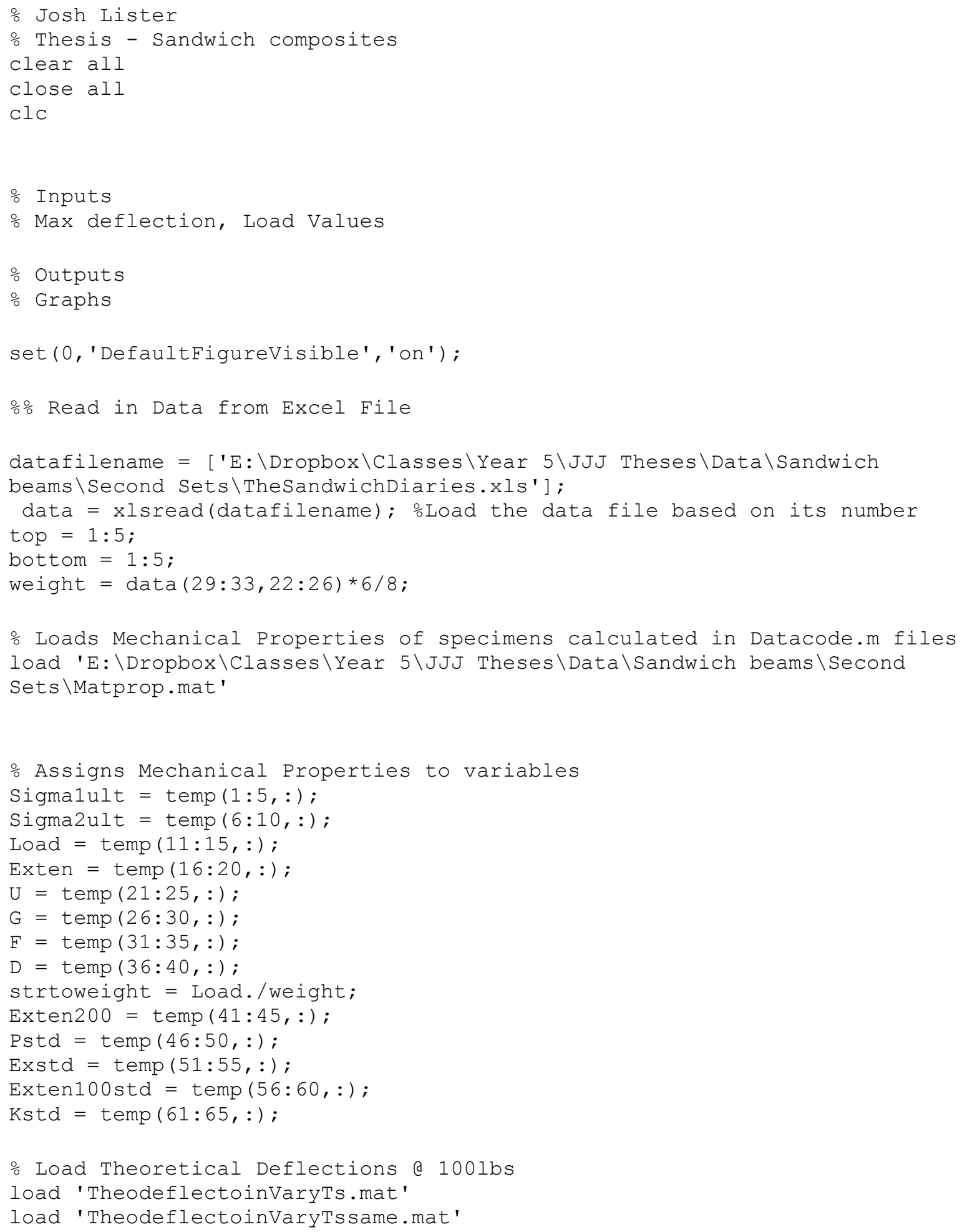




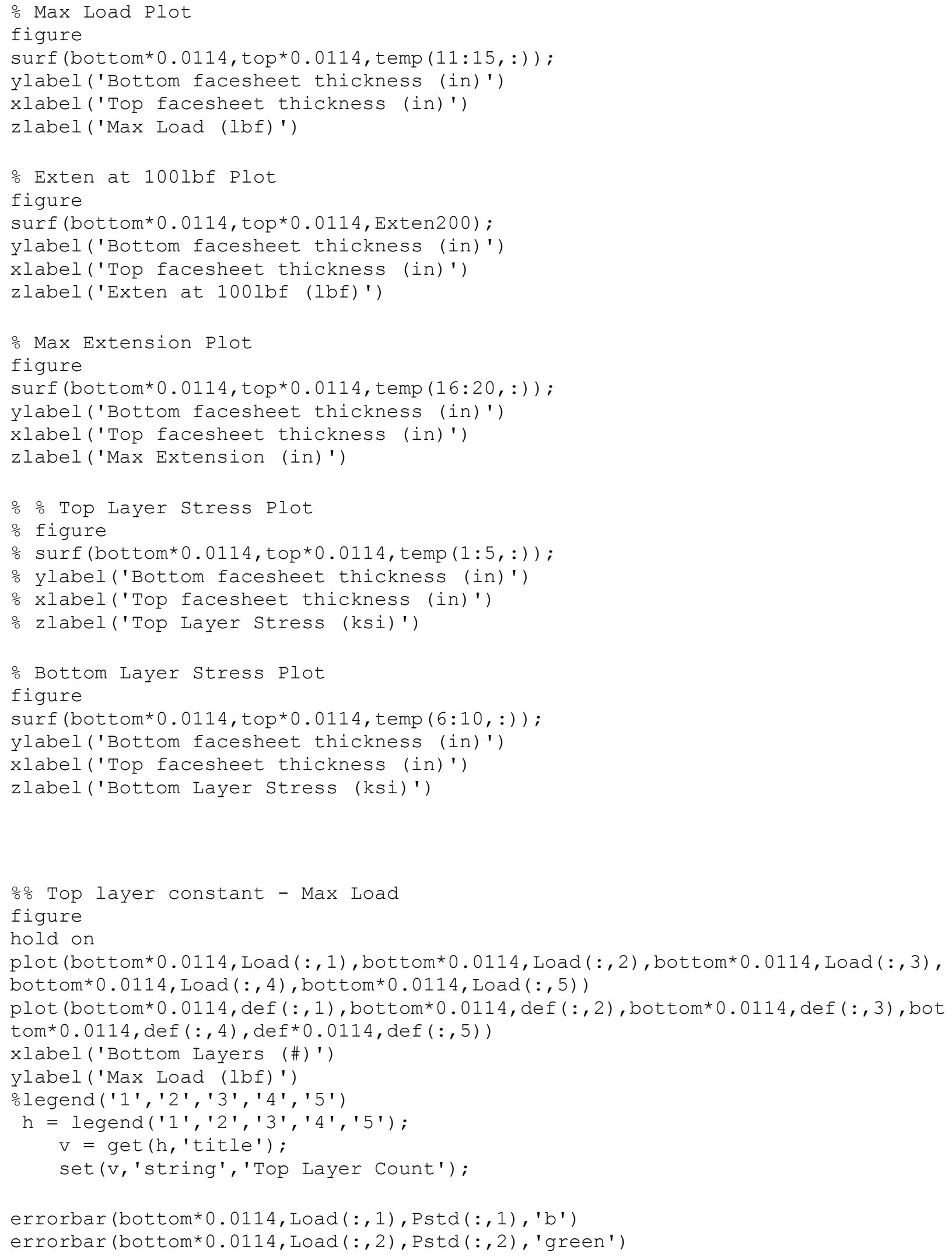




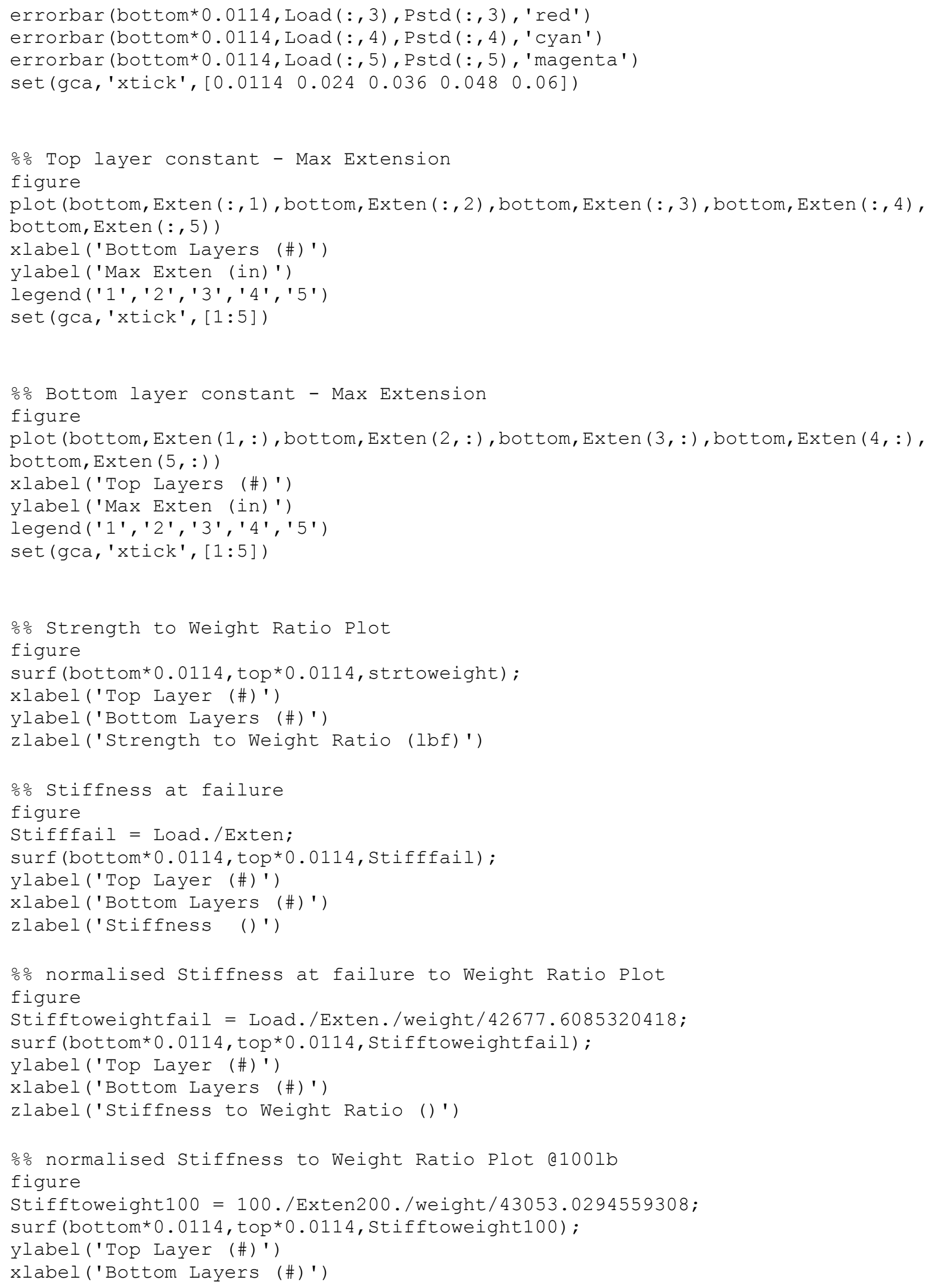




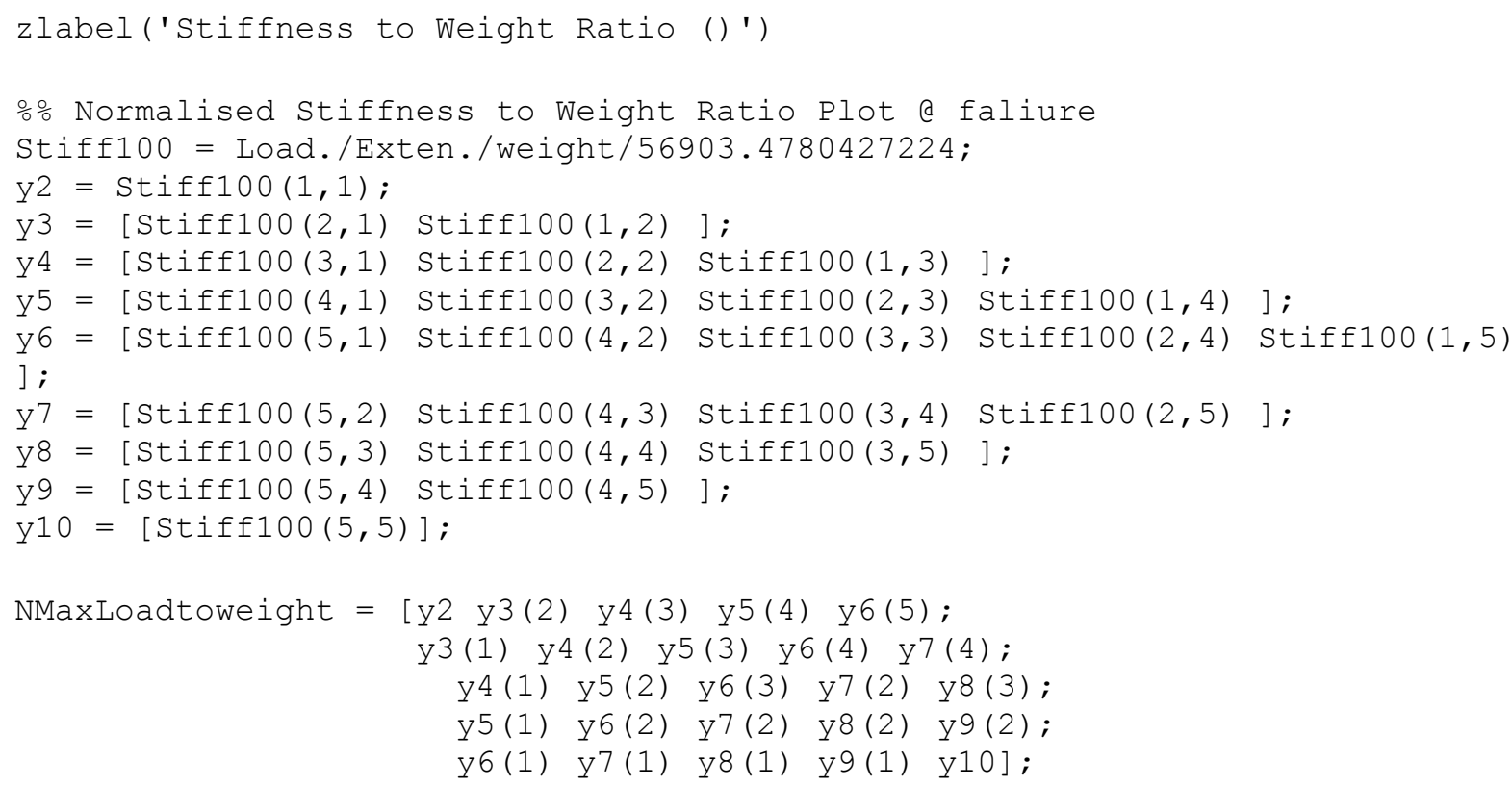




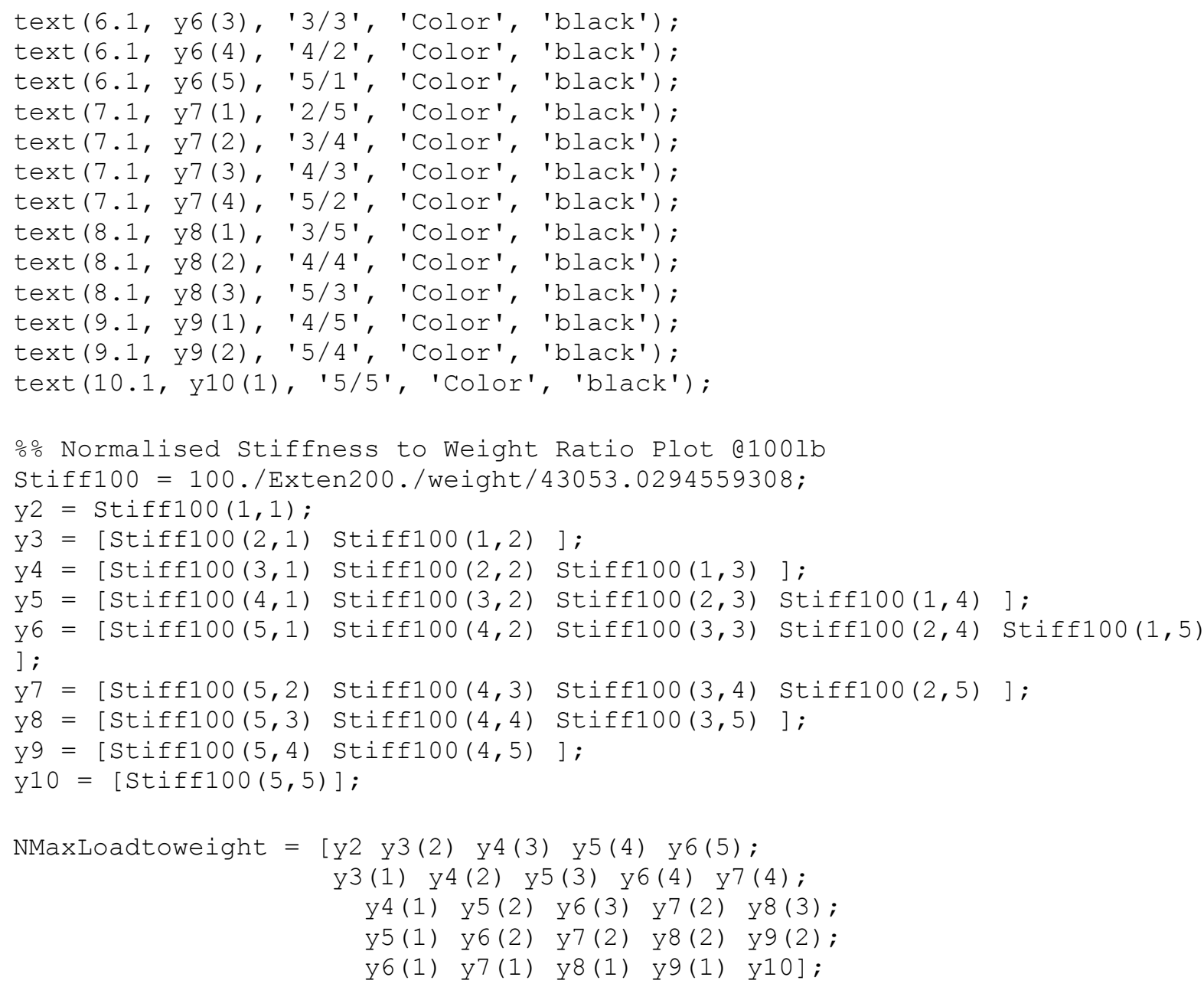




\begin{tabular}{|c|c|c|c|c|}
\hline $\begin{array}{l}\text { text }(2.1, \\
\operatorname{text}(3.1,\end{array}$ & $\begin{array}{l}\text { y2 (1) } \\
\text { y3 (1) }\end{array}$ & $\begin{array}{l}\text { '1/1', } \\
\text { '1/2', }\end{array}$ & $\begin{array}{l}\text { 'Color', } \\
\text { 'Color', }\end{array}$ & 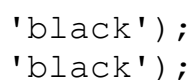 \\
\hline ext $(3.1$, & y3 (2) & '2/1', & 'Color', & 'black') ; \\
\hline ext ( 4.1 , & y4 (1), & $11 / 3 '$, & 'Color', & 'black'); \\
\hline ext ( 4.1 , & $y^{4}(2)$ & '2/2', & 'Color', & 'black'); \\
\hline ext ( 4.1 , & y4 (3) & '3/1', & 'Color', & 'black'); \\
\hline ext (5.1, & $y^{5}(1)$ & '1/4', & 'Color', & 'black'); \\
\hline ext $(5.1$ & y5 (2) & '2/3', & 'Color', & 'black'); \\
\hline ext (5.1 & $y^{5}(3)$ & $13 / 2$ ', & 'Color', & ack'); \\
\hline ext $(5.1$ & $y^{5}(4)$ & '4/1', & 'Color', & 'black'); \\
\hline $\operatorname{ext}(6$. & y6 (1), & '1/5', & 'Color', & $\left.\mathrm{ack}^{\prime}\right)$; \\
\hline ext $(6.1$ & y6 (2), & '2/4', & 'Color', & 'black'); \\
\hline st $(6.1$ & y6 (3), & $13 / 3^{\prime}$, & 'Color', & $\left.\mathrm{k}^{\prime}\right)$; \\
\hline ext $(6.1$ & y6 (4), & $' 4 / 2$ ', & 'Color', & 'black'); \\
\hline$x t(6.1$, & y6 (5), & '5/1', & 'Color', & $\left.\mathrm{ck}^{\prime}\right)$; \\
\hline ext $(7.1$ & $\mathrm{y}^{7}(1)$ & '2/5', & 'Color', & 'black'); \\
\hline $\operatorname{ext}(7.1$ & $y^{7}(2)$ & '3/4', & 'Color', & $\left.\mathrm{ck}^{\prime}\right) ;$ \\
\hline ext ( 7.1 & $y^{7}(3)$ & $14 / 3 '$ & 'Color', & 'black'); \\
\hline$=(7.1$ & $y^{7}(4)$ & '5/2', & 'Color', & $\left.\mathrm{ck}^{\prime}\right) ;$ \\
\hline ext $(8$. & y8 (1), & '3/5', & 'Color', & 'black'); \\
\hline$(8$. & y8 (2) & ' 4/4', & 'Color', & ack'); \\
\hline$(8.1$ & y8 (3) & '5/3', & 'Color', & 'black'); \\
\hline & $y^{9}(1)$ & '4/5', & 'Color', & $\left.\mathrm{k}^{\prime}\right)$; \\
\hline 0 & $y^{9}(2)$ & '5/4', & 'Color', & $\left.k^{\prime}\right)$ \\
\hline
\end{tabular}

응 Normalised Max Load/Weight

layer2 = Load $(1,1)$;

layer $3=[\operatorname{Load}(2,1) \operatorname{Load}(1,2)]$;

layer4 $=[\operatorname{Load}(3,1) \operatorname{Load}(2,2) \operatorname{Load}(1,3)]$;

layer5 $=[\operatorname{Load}(4,1) \operatorname{Load}(3,2) \operatorname{Load}(2,3) \operatorname{Load}(1,4)]$;

layer6 $=[\operatorname{Load}(5,1) \operatorname{Load}(4,2) \operatorname{Load}(3,3) \operatorname{Load}(2,4) \operatorname{Load}(1,5)]$;

layer $7=[\operatorname{Load}(5,2) \operatorname{Load}(4,3) \operatorname{Load}(3,4) \operatorname{Load}(2,5)]$;

layer $8=[\operatorname{Load}(5,3) \operatorname{Load}(4,4) \operatorname{Load}(3,5)]$;

layer $9=[\operatorname{Load}(5,4) \operatorname{Load}(4,5)]$;

layer10 = $[\operatorname{Load}(5,5)]$;

weightavg2 = weight $(1,1)$;

weightavg3 = [weight $(2,1)$ weight $(1,2)]$;

weightavg $4=$ [weight $(3,1)$ weight $(2,2)$ weight $(1,3)$ ];

weightavg5 $=[$ weight $(4,1)$ weight $(3,2)$ weight $(2,3)$ weight $(1,4)]$;

weightavg $6=$ [weight $(5,1)$ weight $(4,2)$ weight $(3,3)$ weight $(2,4)$ weight (1,5) ] ;

weightavg $7=[$ weight $(5,2)$ weight $(4,3)$ weight $(3,4)$ weight $(2,5)]$;

weightavg $8=[$ weight $(5,3)$ weight $(4,4)$ weight $(3,5)]$;

weightavg $9=[$ weight $(5,4)$ weight $(4,5)]$;

weightavg10 = weight $(5,5)$;

Normalisedweight $=$ [weightavg2 weightavg3 weightavg4 weightavg5 weightavg6 weightavg7 weightavg8 weightavg9 weightavg10]./weightavg2;

o Calculates Normalised (to weakest 1/5) Load at failure/weight

weak =layer6./weightavg6; $1 / 5$ is weakest

y2 =layer2./weightavg2./weak (1);

y3 =layer3./weightavg3./weak (1); 


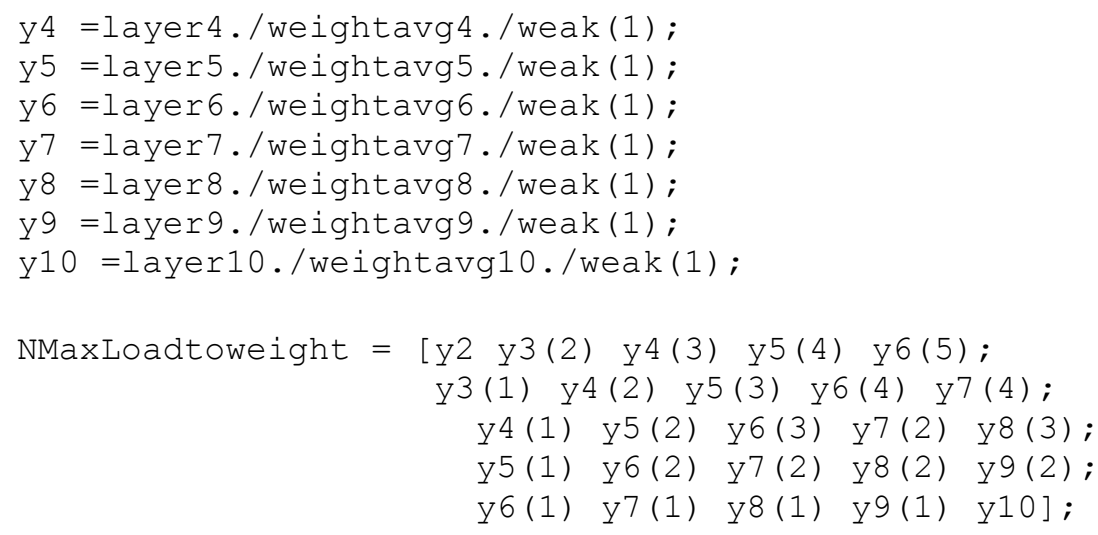




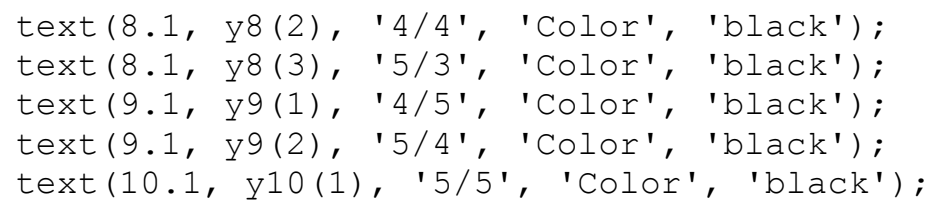




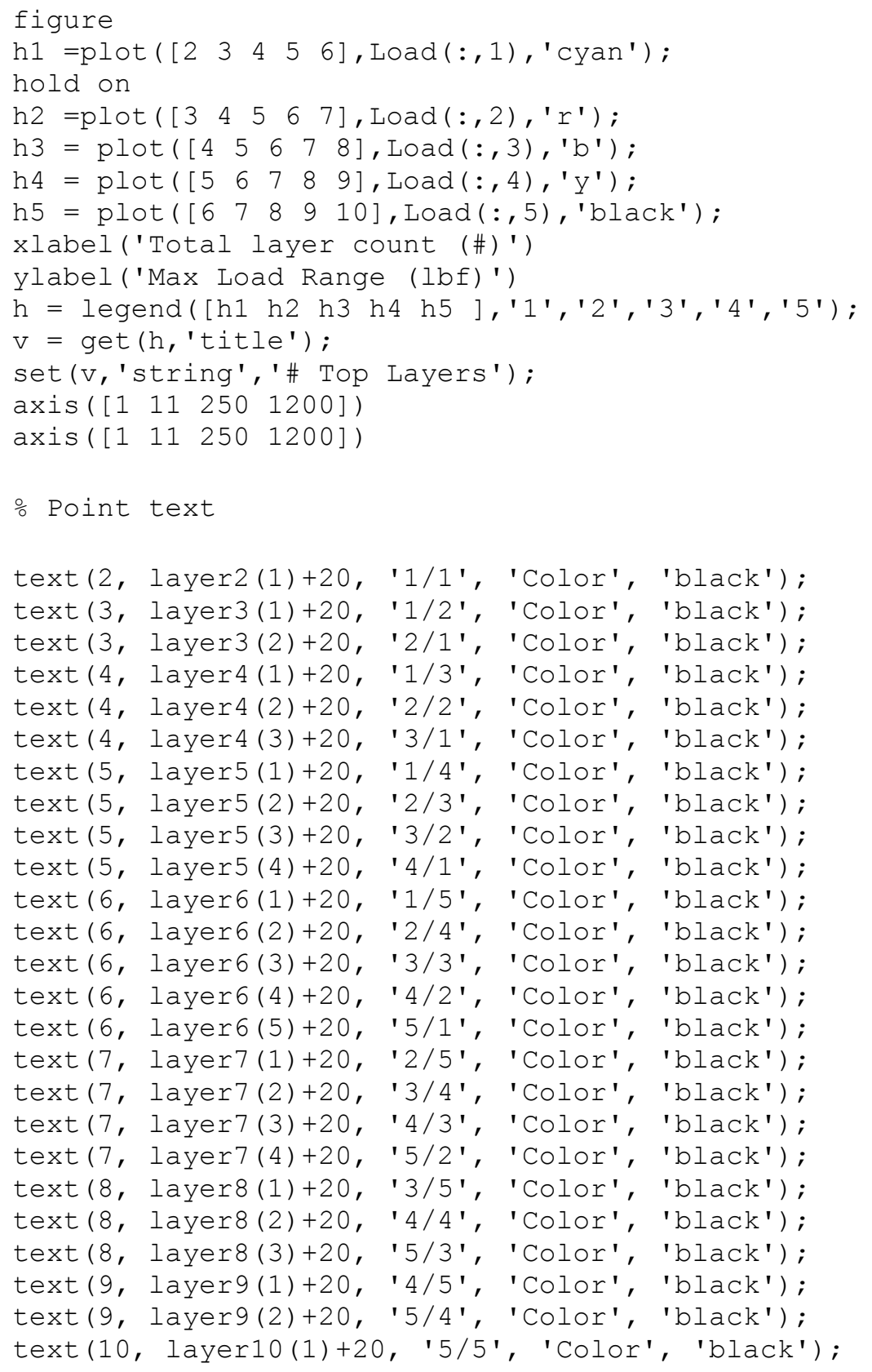

응 Max Load Vs Weight

weightavg2 = weight $(1,1) ;$

weightavg3 = [weight $(2,1)$ weight $(1,2)]$;

weightavg $4=[$ weight $(3,1)$ weight $(2,2)$ weight $(1,3)]$;

weightavg5 = [weight $(4,1)$ weight $(3,2)$ weight $(2,3)$ weight $(1,4)]$; 
weightavg $6=$ [weight $(5,1)$ weight $(4,2)$ weight $(3,3)$ weight $(2,4)$ weight (1, 5) ] ; weightavg7 = [weight $(5,2)$ weight $(4,3)$ weight $(3,4)$ weight $(2,5)$;

weightavg $8=$ [weight $(5,3)$ weight $(4,4)$ weight $(3,5)$ ];

weightavg $9=[$ weight $(5,4)$ weight $(4,5)$ ];

weightavg10 = weight $(5,5)$;

Normalisedweight $=$ [weightavg2 weightavg3 weightavg4 weightavg5 weightavg6 weightavg7 weightavg8 weightavg9 weightavg10]./weightavg2;

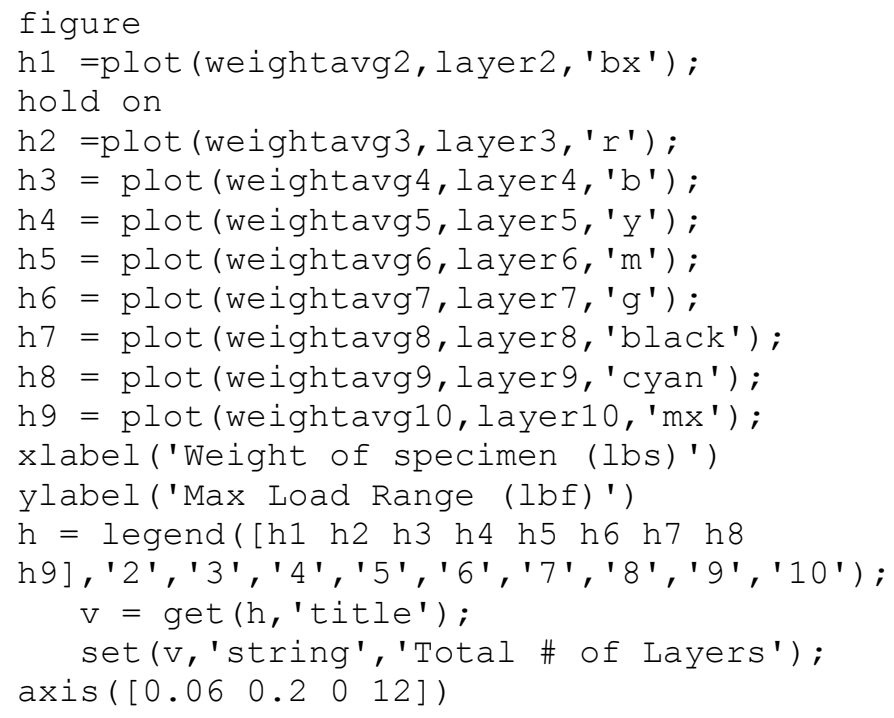

Point text

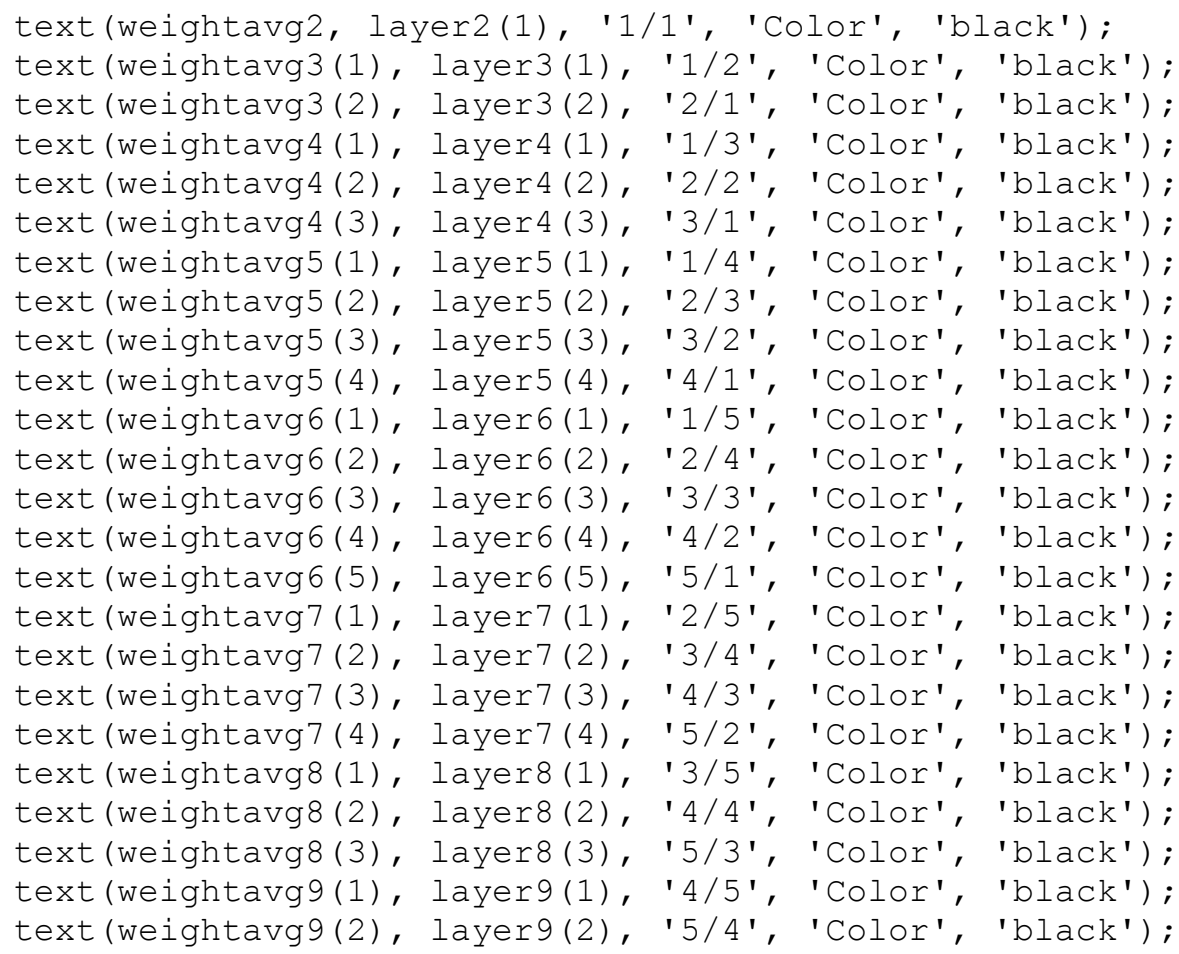


text (weightavg10(1), layer10(1), '5/5', 'Color', 'black');

응 Maximum Extension of an Average american male (100lbs)

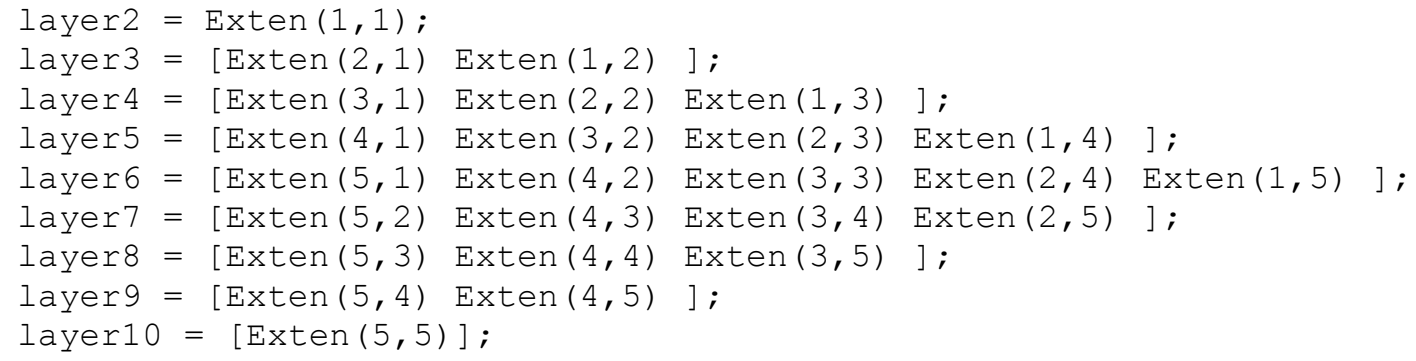

\section{figure}

h1 =plot ([2], layer2, 'bx');

hold on

h2 =plot ( [ 3 3 $]$, layer3, 'r');

h3 $=\operatorname{plot}\left(\left[\begin{array}{lll}4 & 4 & 4\end{array}\right]\right.$, layer4, 'b');

$\mathrm{h} 4=\operatorname{plot}\left(\left[\begin{array}{llll}5 & 5 & 5 & 5\end{array}\right]\right.$, layer5, 'y') ;

h5 $=\operatorname{plot}\left(\left[\begin{array}{lllll}6 & 6 & 6 & 6 & 6\end{array}\right]\right.$, layer6, 'm');

$\mathrm{h} 6=\operatorname{plot}\left(\left[\begin{array}{llll}7 & 7 & 7 & 7\end{array}\right]\right.$, layer 7, ' $^{\prime}$ ') ;

$\mathrm{h} 7=\operatorname{plot}\left(\left[\begin{array}{lll}8 & 8 & 8\end{array}\right]\right.$, layer8,'black') ;

h8 = plot ([9 9], layer9, 'cyan');

h9 $=$ plot ([10], layer10, 'mx');

xlabel ('Total layer count (\#)')

ylabel ('Max Exten Range (lbf)')

$h=$ legend ( [h1 h2 h3 h4 h5 h6 h7 h8

h9] ,'1','1.2','1.43','1.65', '1.88','2.09','2.09','2.3','2.52', '2.72');

$\mathrm{v}=\operatorname{get}(\mathrm{h}$, 'title');

set (v, 'string', 'Normalised Weight');

axis ( [ 1110.070 .2$])$

응 text

\begin{tabular}{|c|c|c|c|c|}
\hline $\operatorname{ext}(2.1$, & 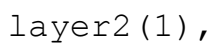 & 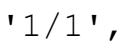 & & \\
\hline$x L$ & layer3 (1), & '1/2', & 'Color', & 'black' ) \\
\hline$x t(3.1$ & layer3 (2), & '2/1', & 'Color', & 'black') \\
\hline ( & layer4 (1), & $1 / 3$ ', & 'Color', & $\left.\mathrm{Ck}^{\prime}\right)$ \\
\hline$x t(4$ & layer4(2), & '2/2', & 'Color', & $\left.\mathrm{ack}^{\prime}\right)$ \\
\hline - & layer4 (3), & '3/1', & or', & $\mathrm{b}$ \\
\hline$x t$ & layer5(1), & '1/4', & 'Color', & $\left.\mathrm{ck}^{\prime}\right)$ \\
\hline oxt & layer5 (2), & $12 / 3^{\prime}$, & $r^{\prime}$, & 'b \\
\hline ext ( & layer5 (3), & '3/2', & or', & $\left.\mathrm{ck}^{\prime}\right)$ \\
\hline ext ( & layer5 (4), & '4/1', & $r^{\prime}$, & $1 \mathrm{~b}$ \\
\hline$\partial x$ & layer6(1), & '1/5', & Lor', & $\left.k^{\prime}\right)$ \\
\hline ex & layer6(2), & '2/4', & $r^{\prime}$, & $\left.\mathrm{K}^{\prime}\right)$ \\
\hline$=($ & layer6(3), & $13 / 31$, & or', & $\left.k^{\prime}\right)$ \\
\hline & layer6(4), & '4/2', & $r^{\prime}$, & '] \\
\hline & layer & '5/1', & $r^{\prime}$, & \\
\hline 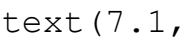 & layer7 (1) & '2/5', & & \\
\hline
\end{tabular}




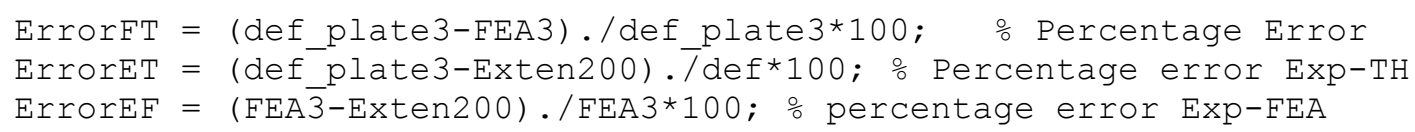




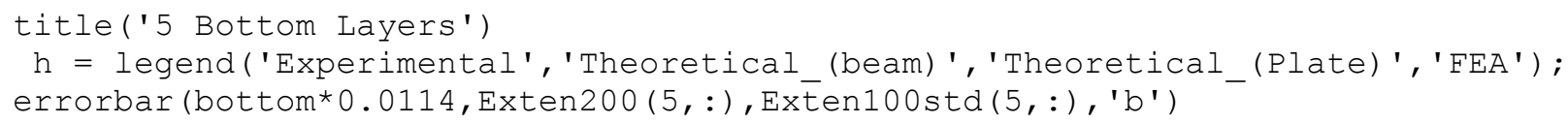

\section{A.8.11. Minimum and Maximum Core weight for Optimum Strength with respect to minimum weight}

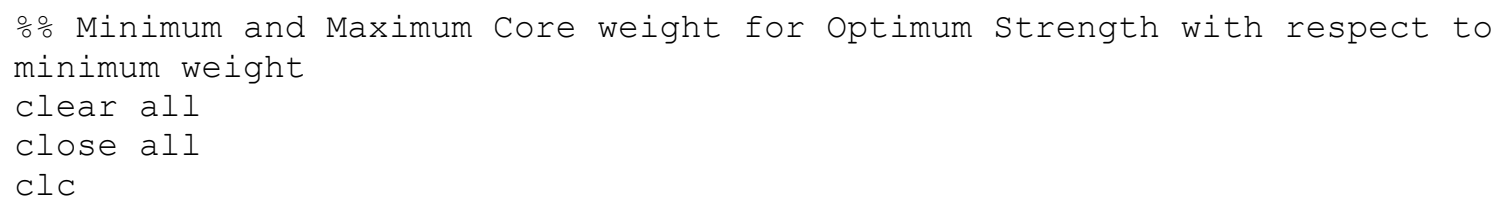




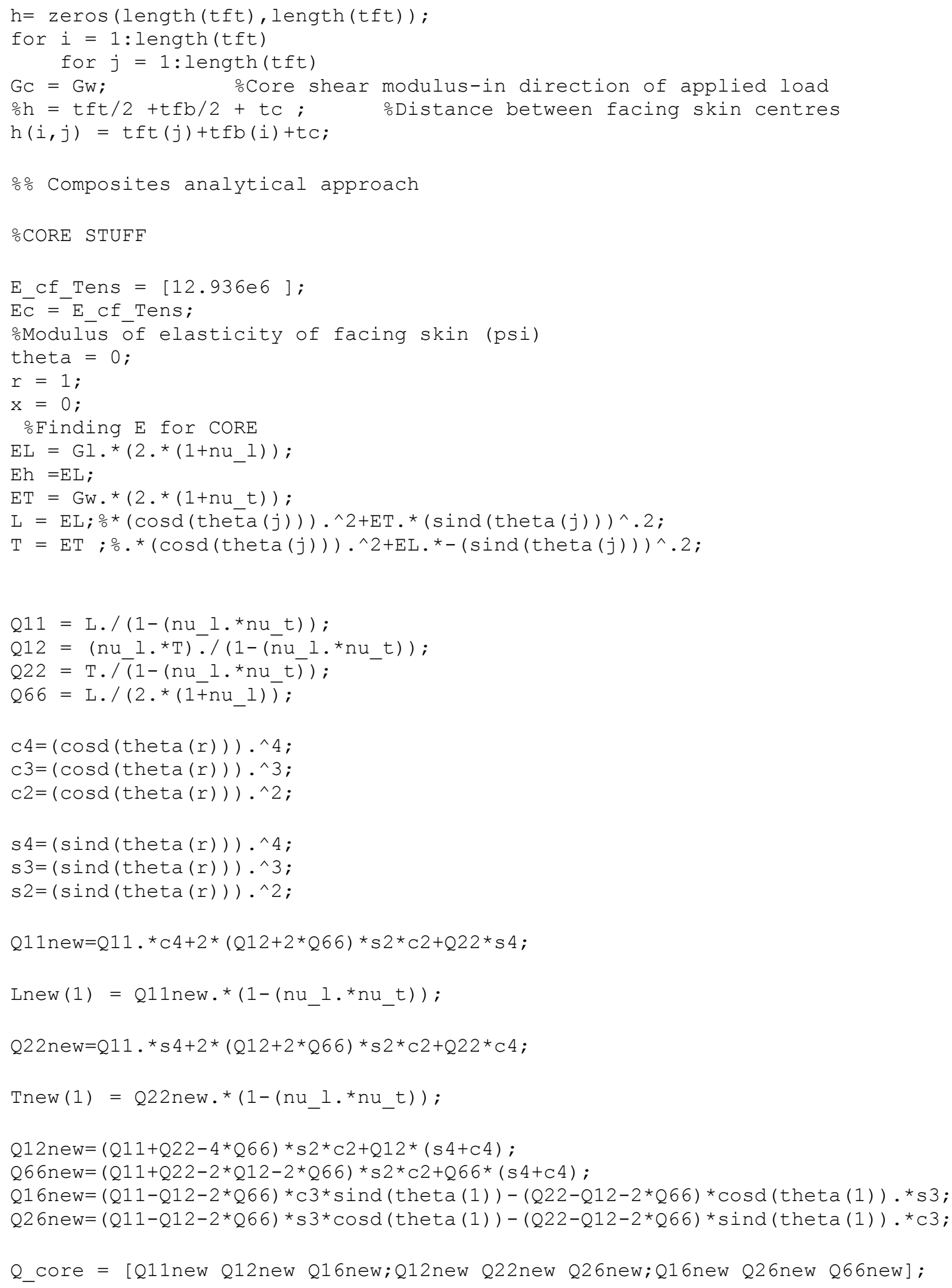




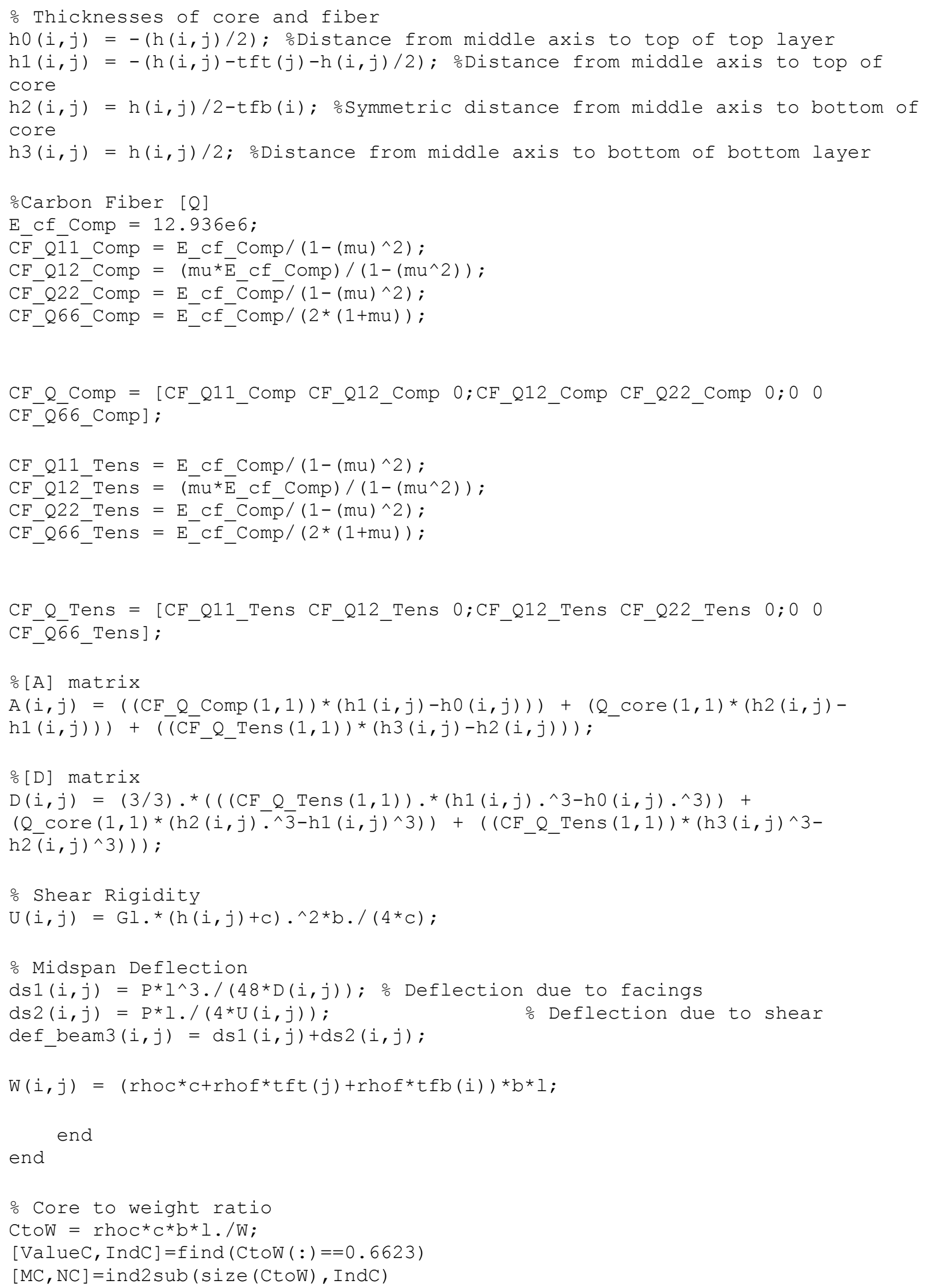




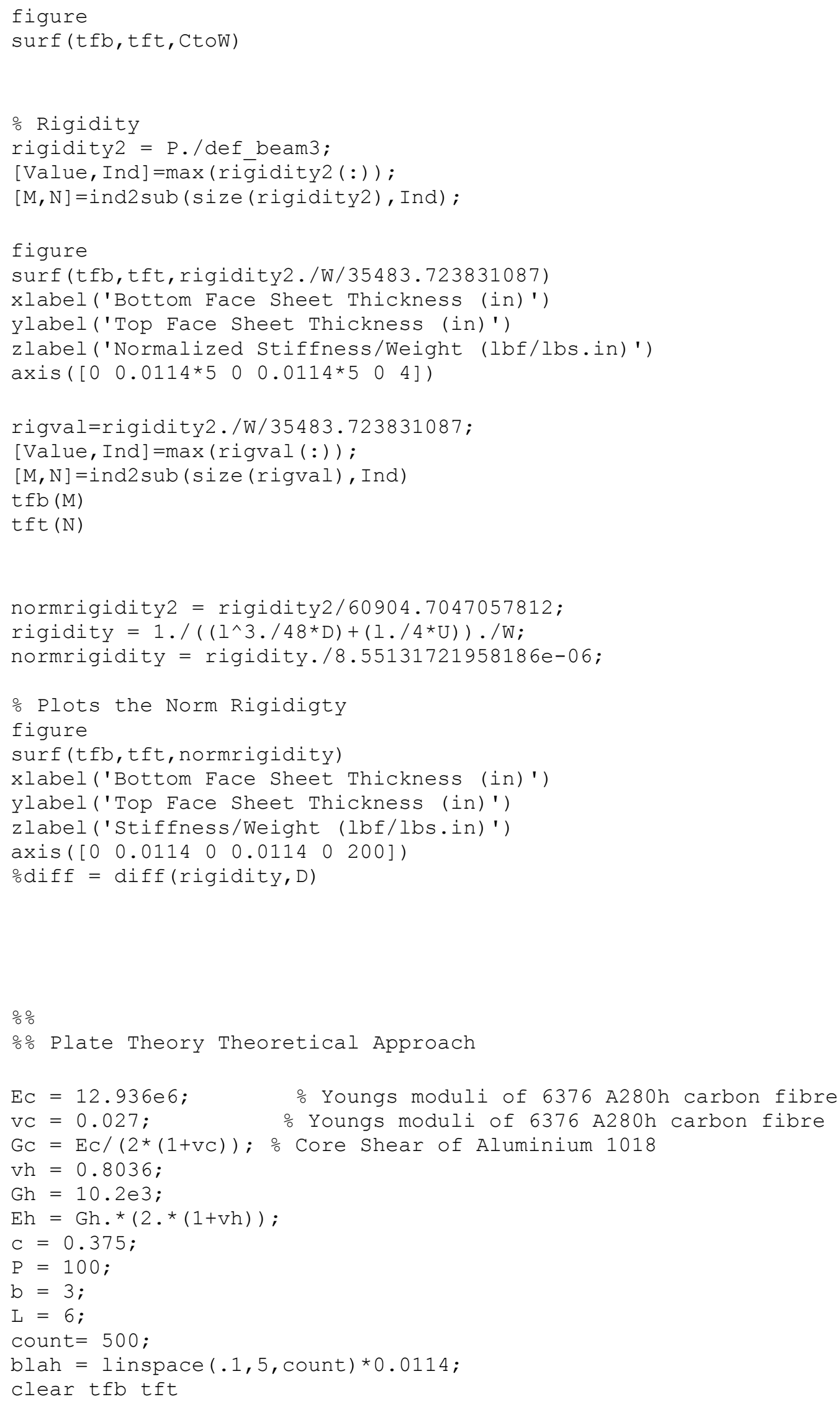




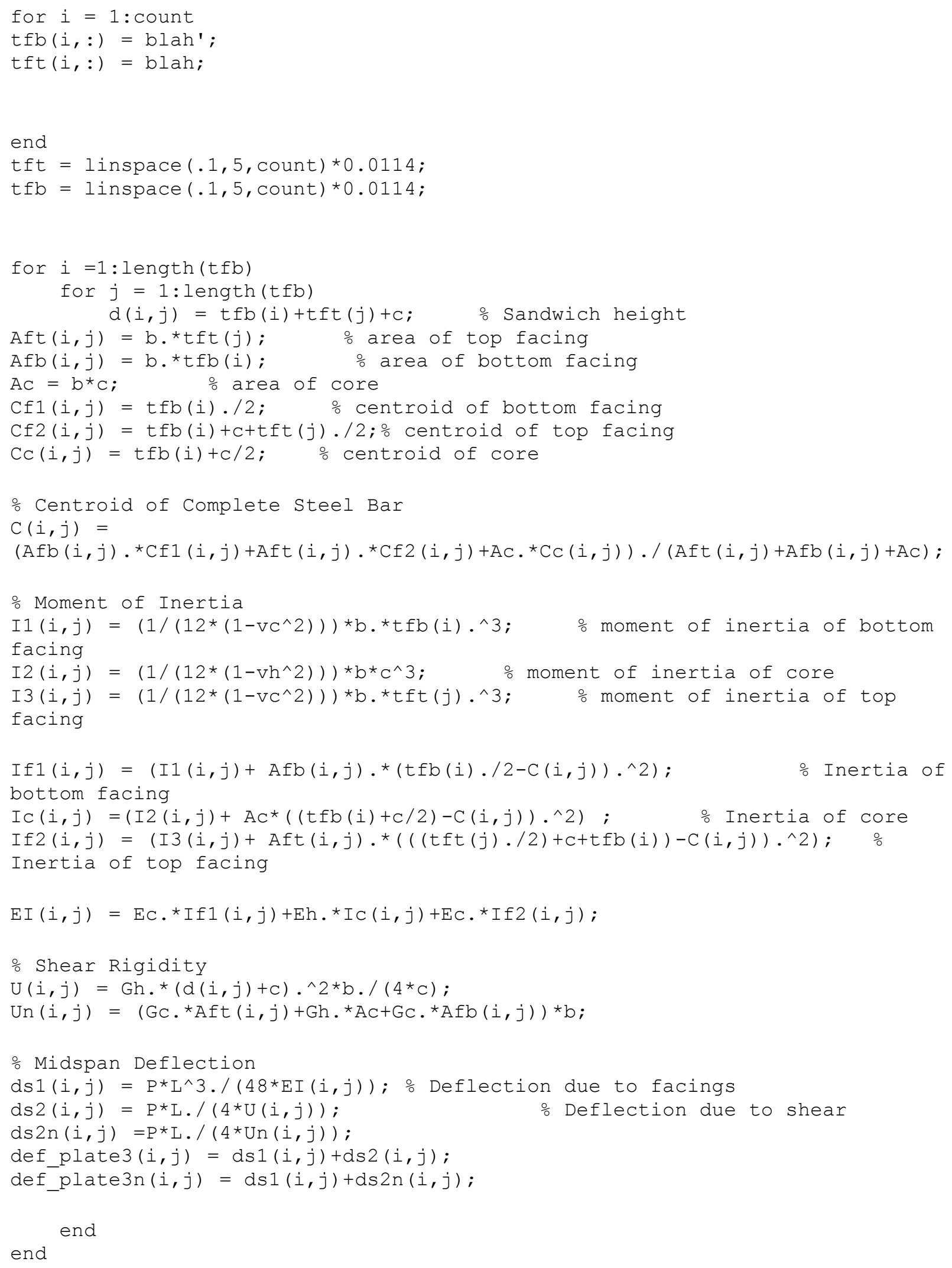




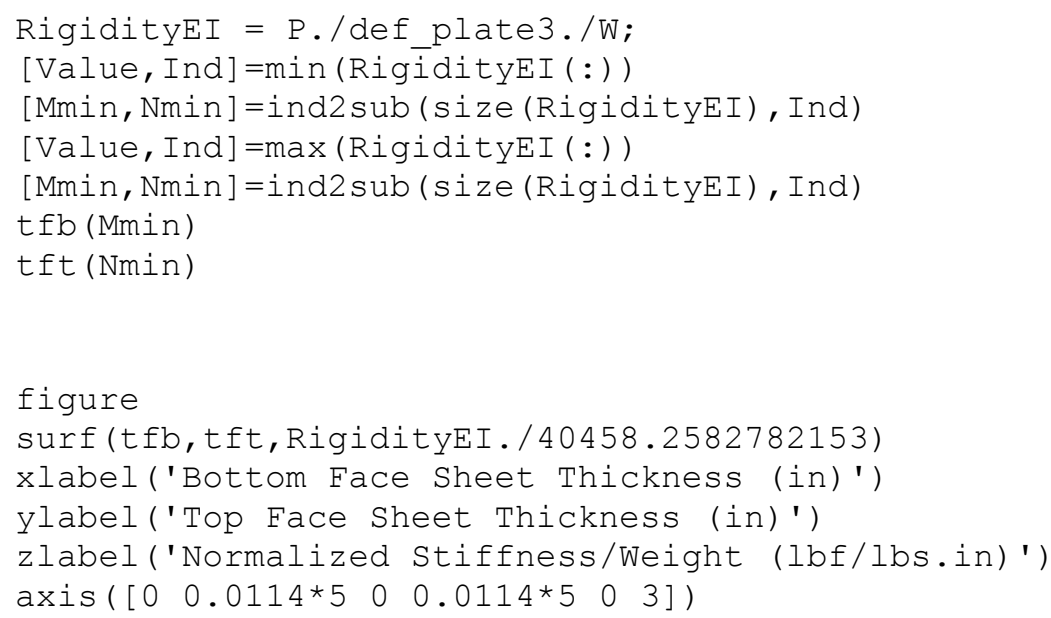

\section{A.8.12. Theoretical Beam and Plate Theory values for varying facing thickness}

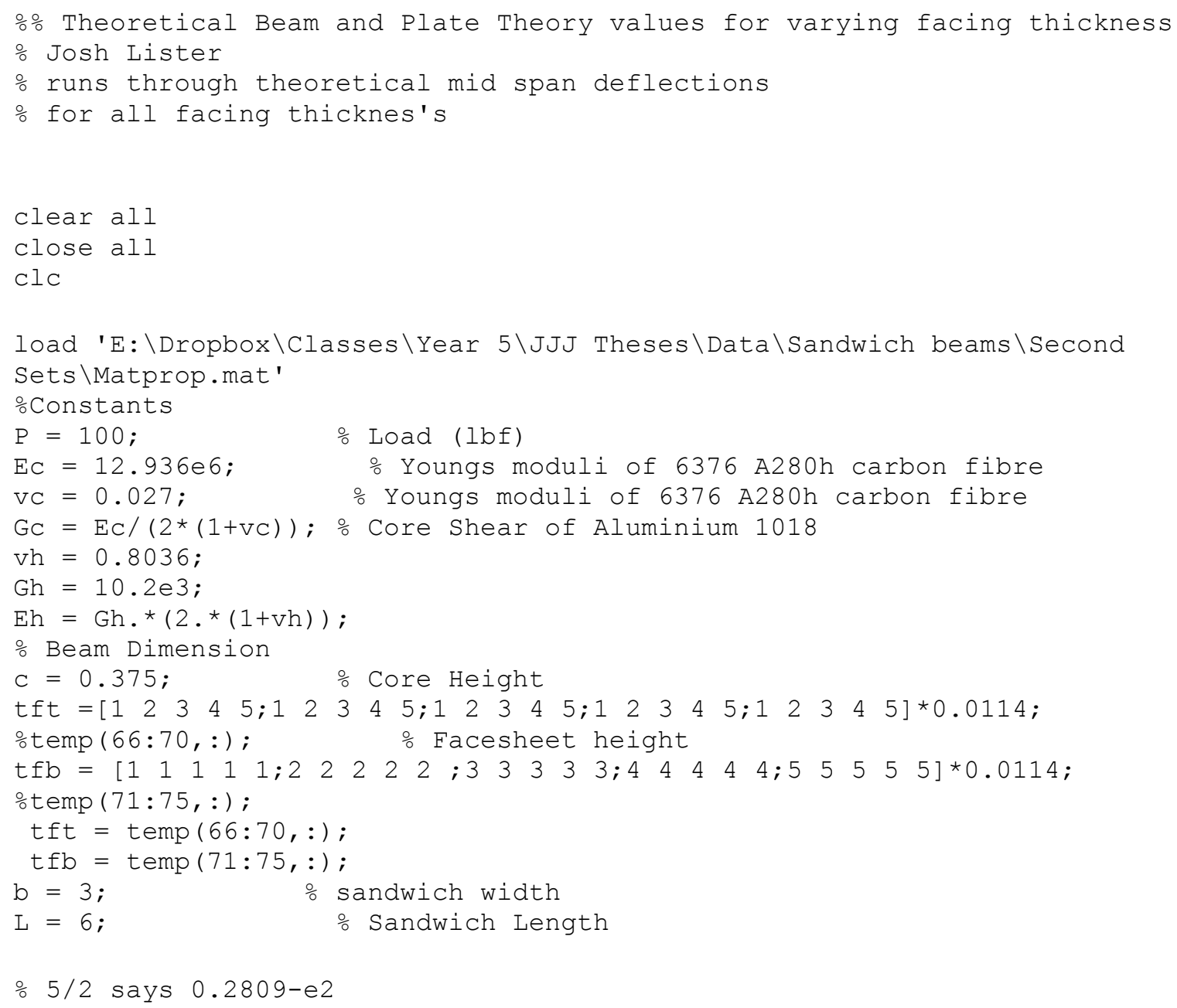




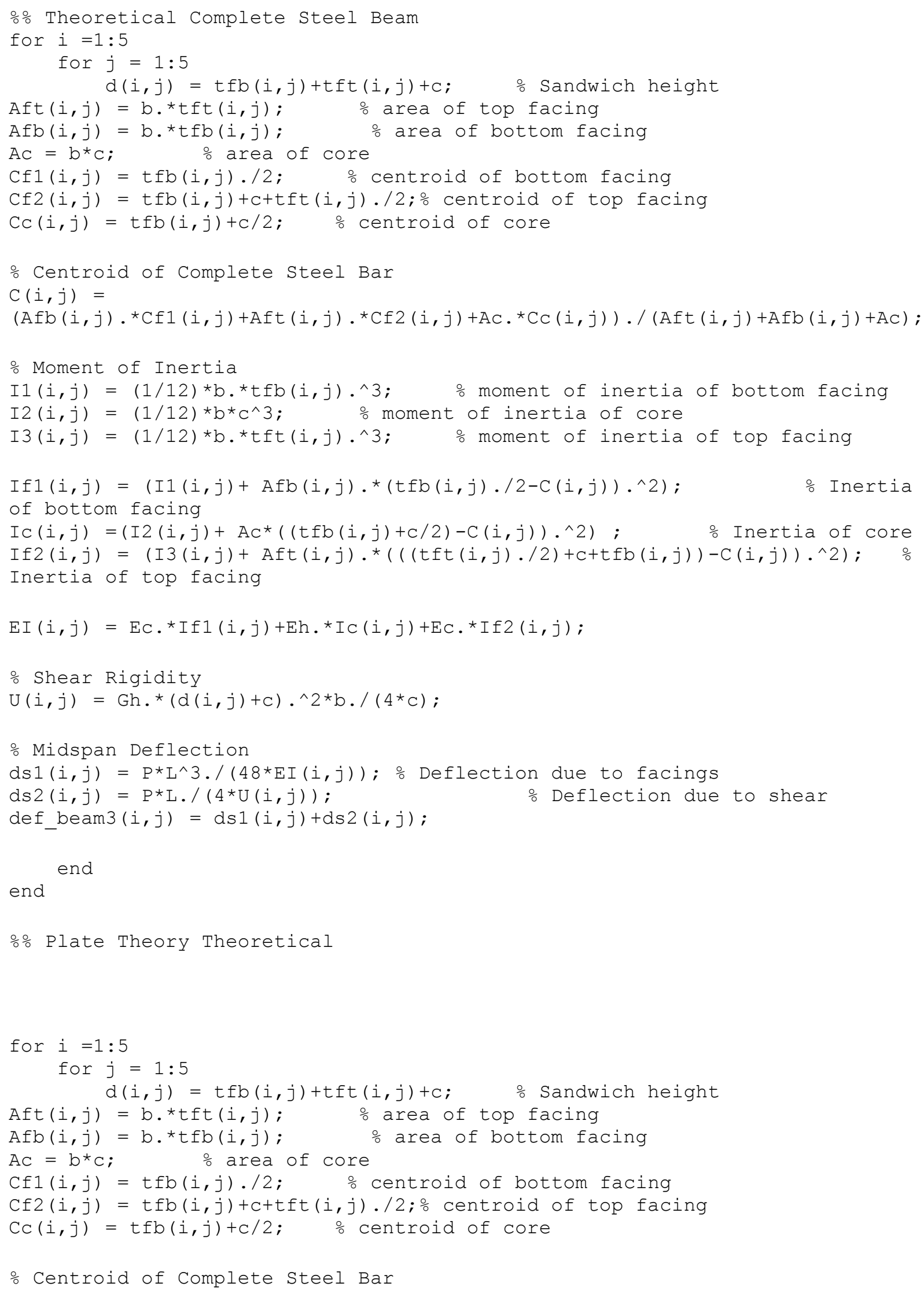




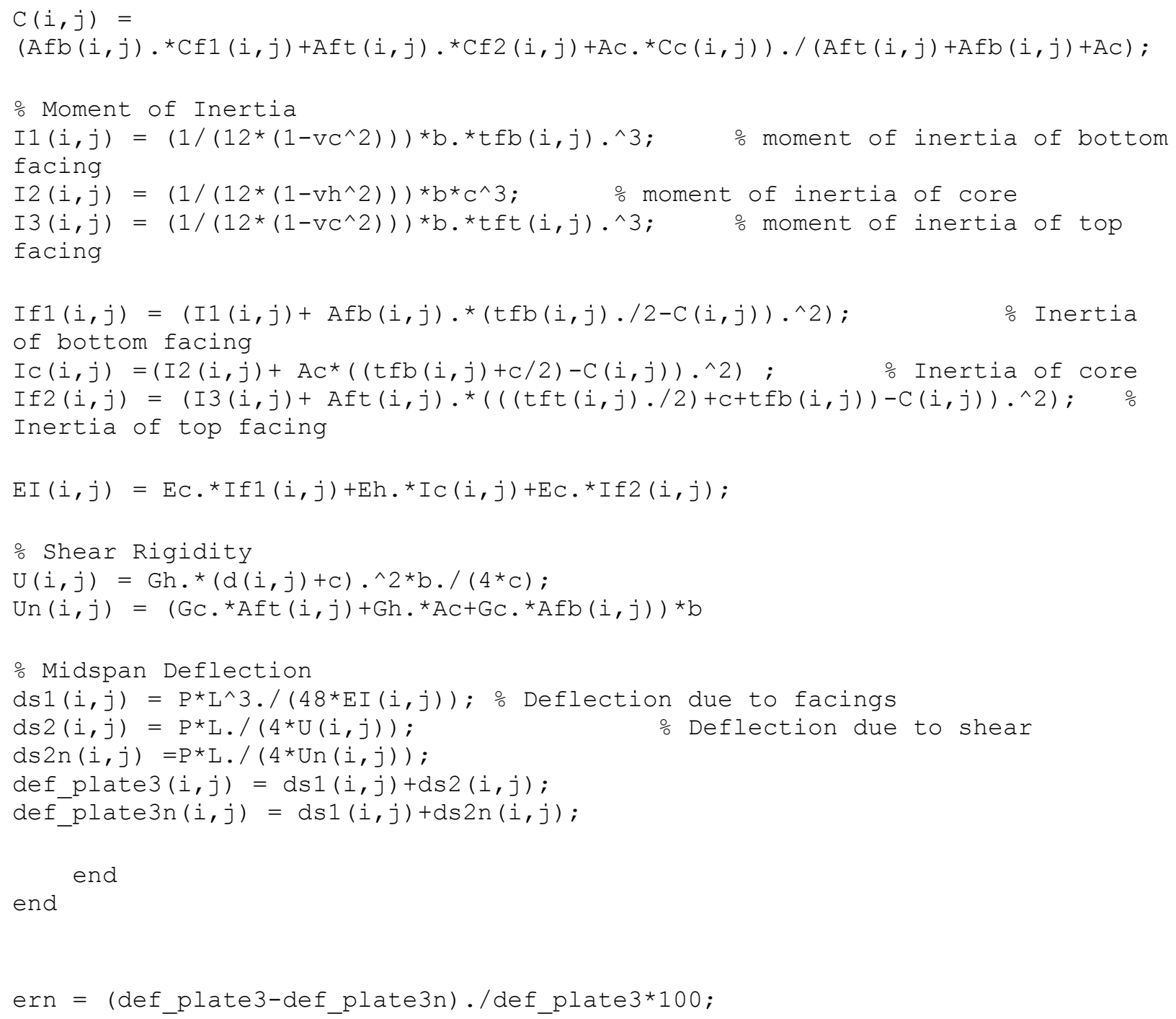

\section{A.8.13. Polyfit Plots for Experimental, FEA Data}

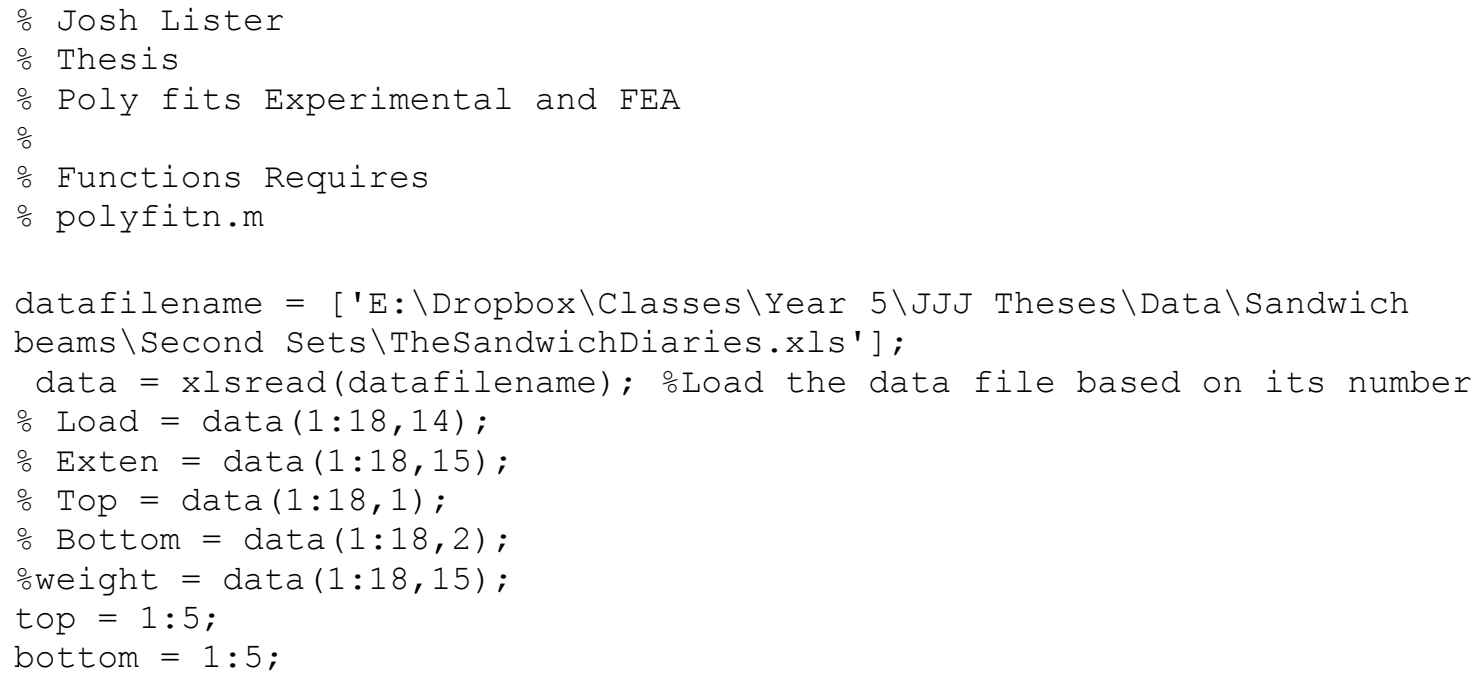


ㄴoad $=\operatorname{data}(11: 15,22: 26)$;

을 $\operatorname{Exten}=\operatorname{data}(20: 24,22: 26)$;

weight $=\operatorname{data}(29: 33,22: 26) * 6 / 8$;

\% strtoweight $=$ Load1./weight1;

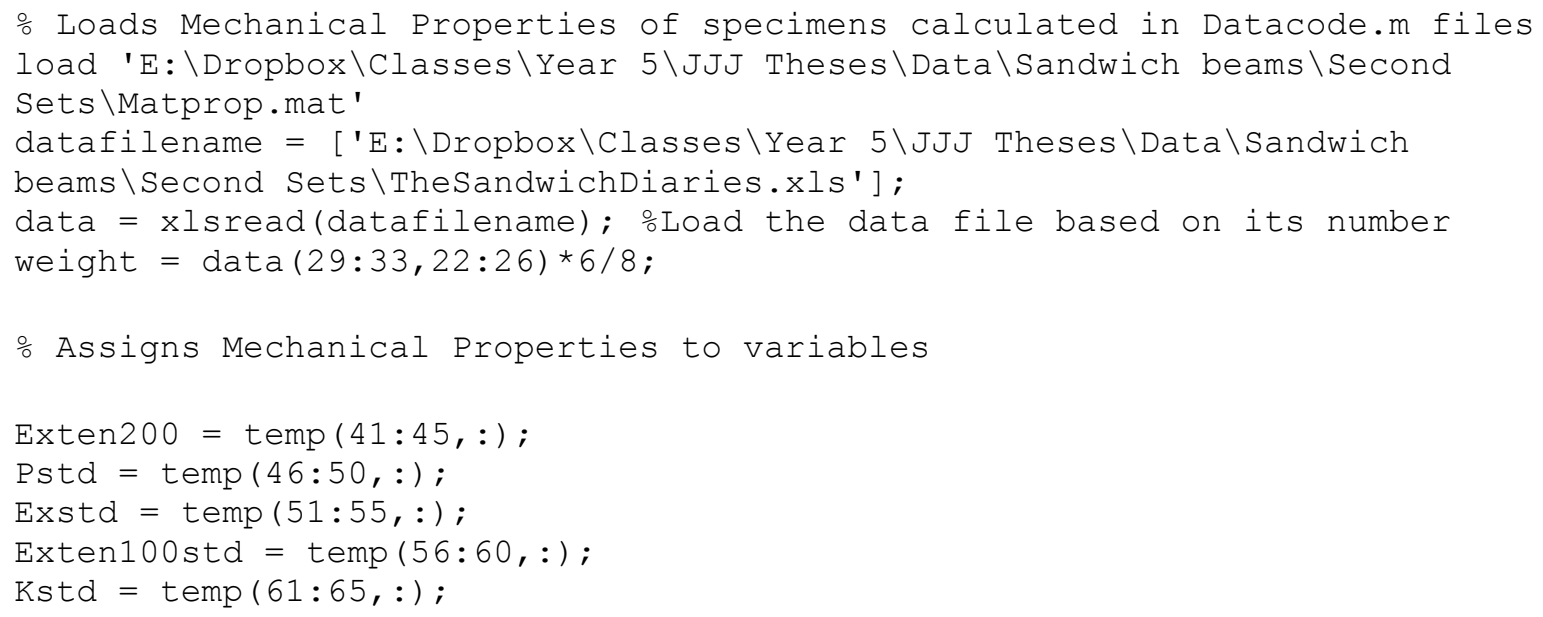




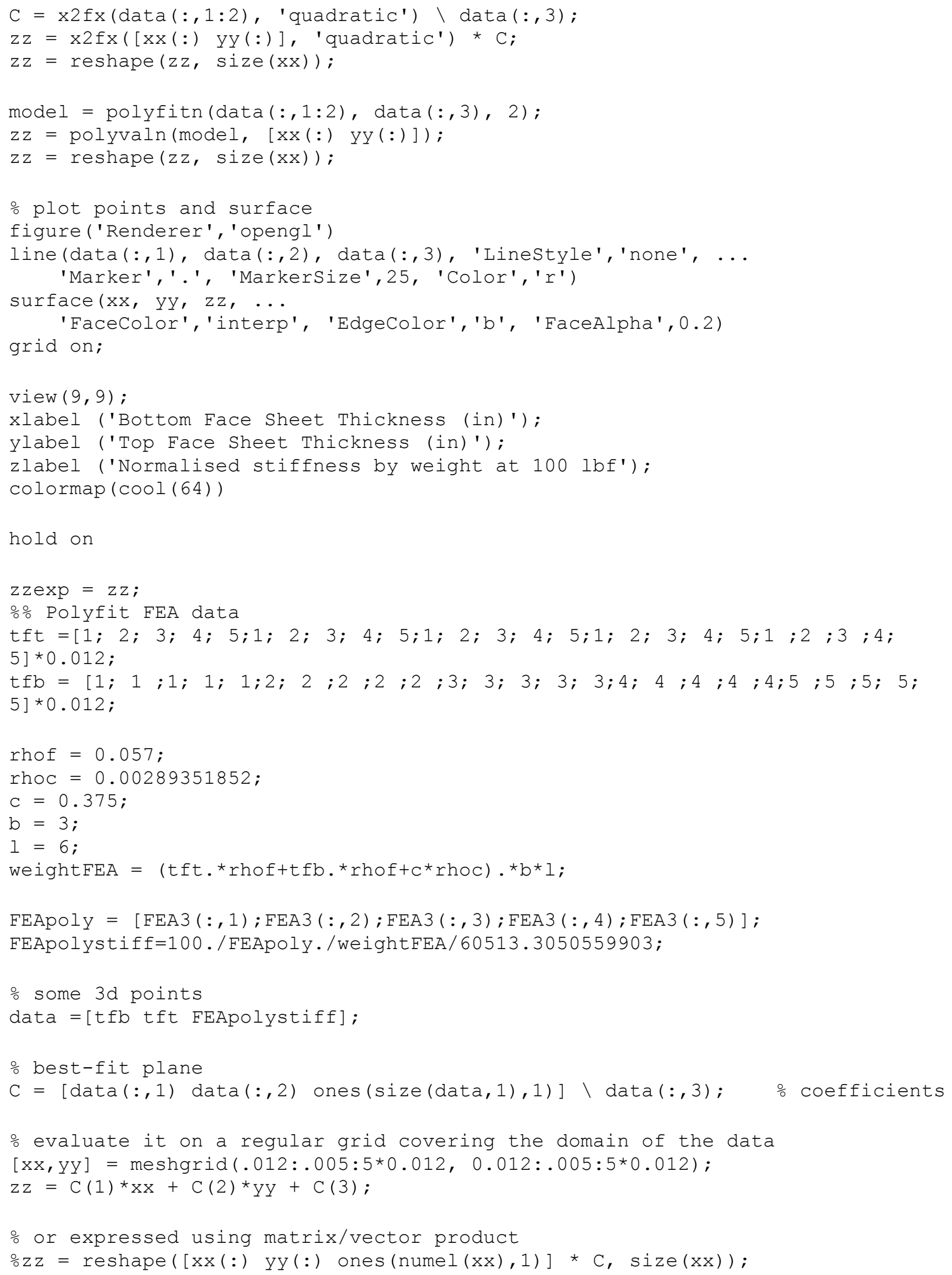




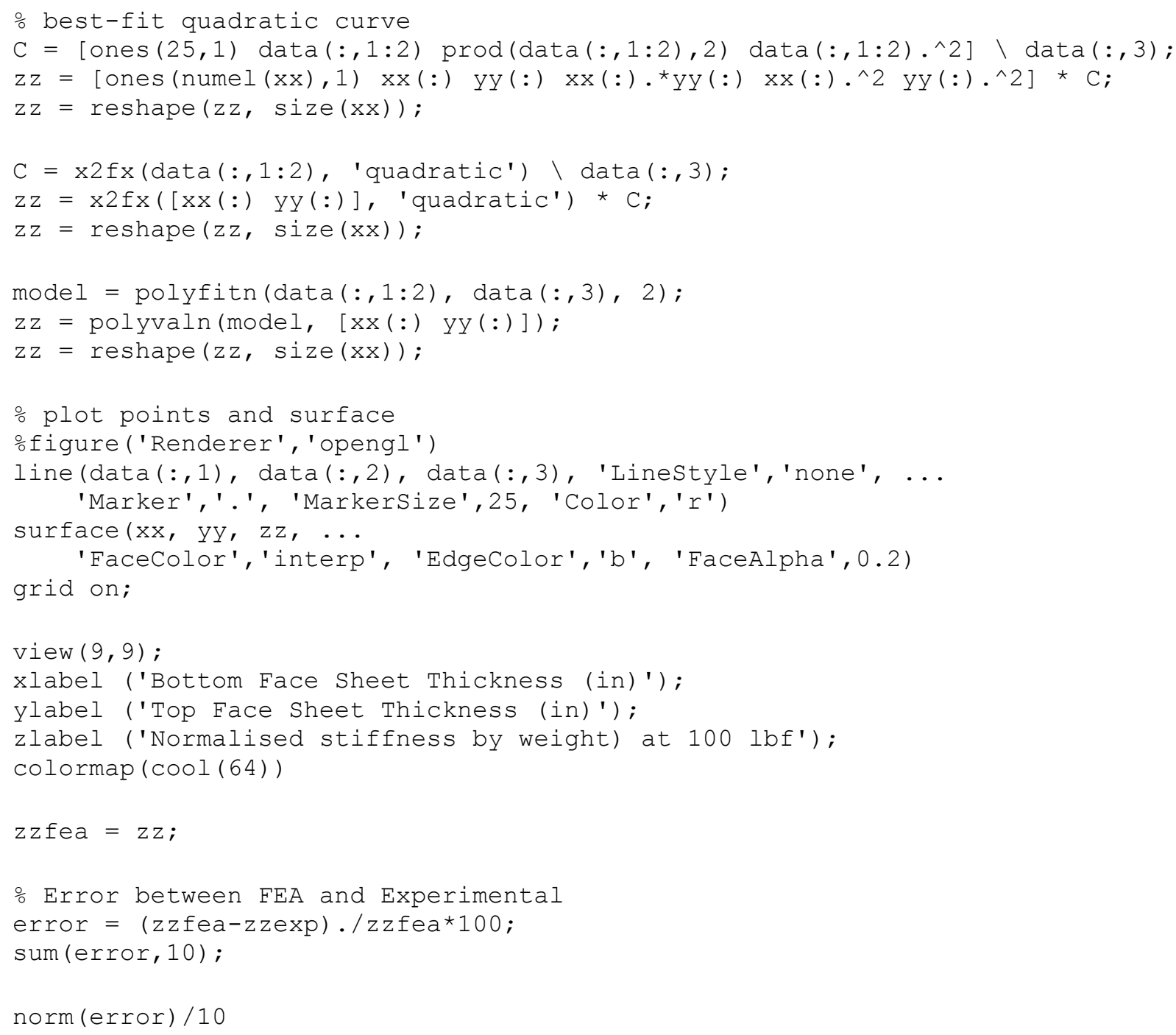

\section{A.8.14. 3D Polyfit function for Data Points}

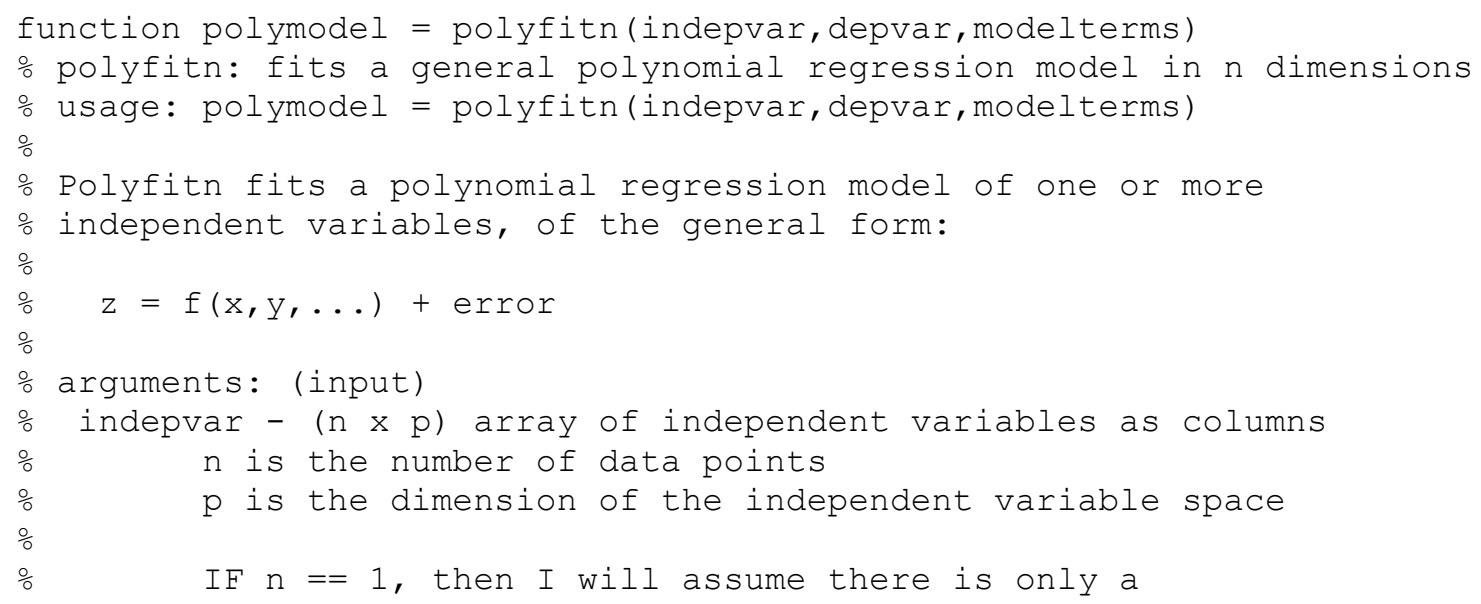




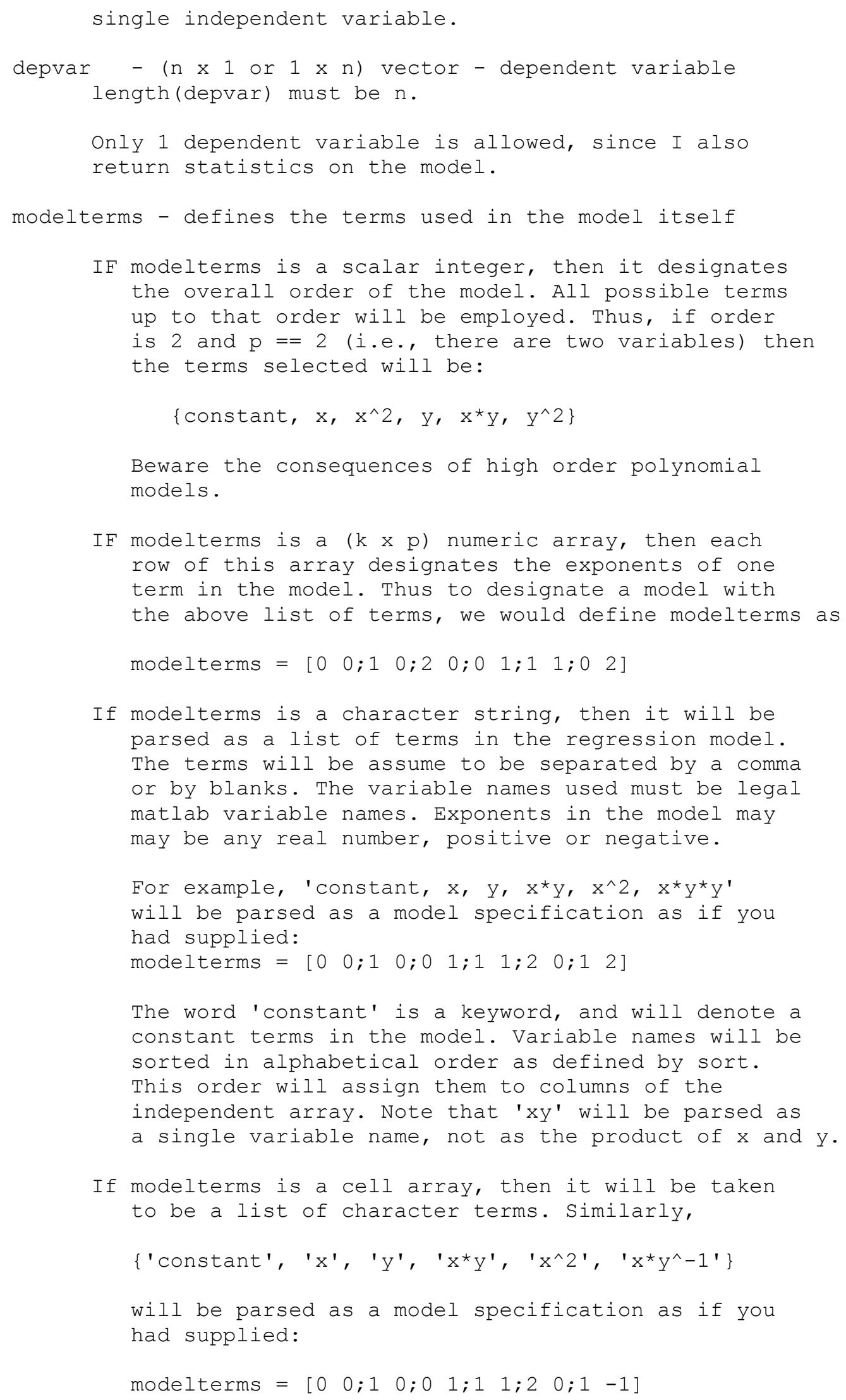




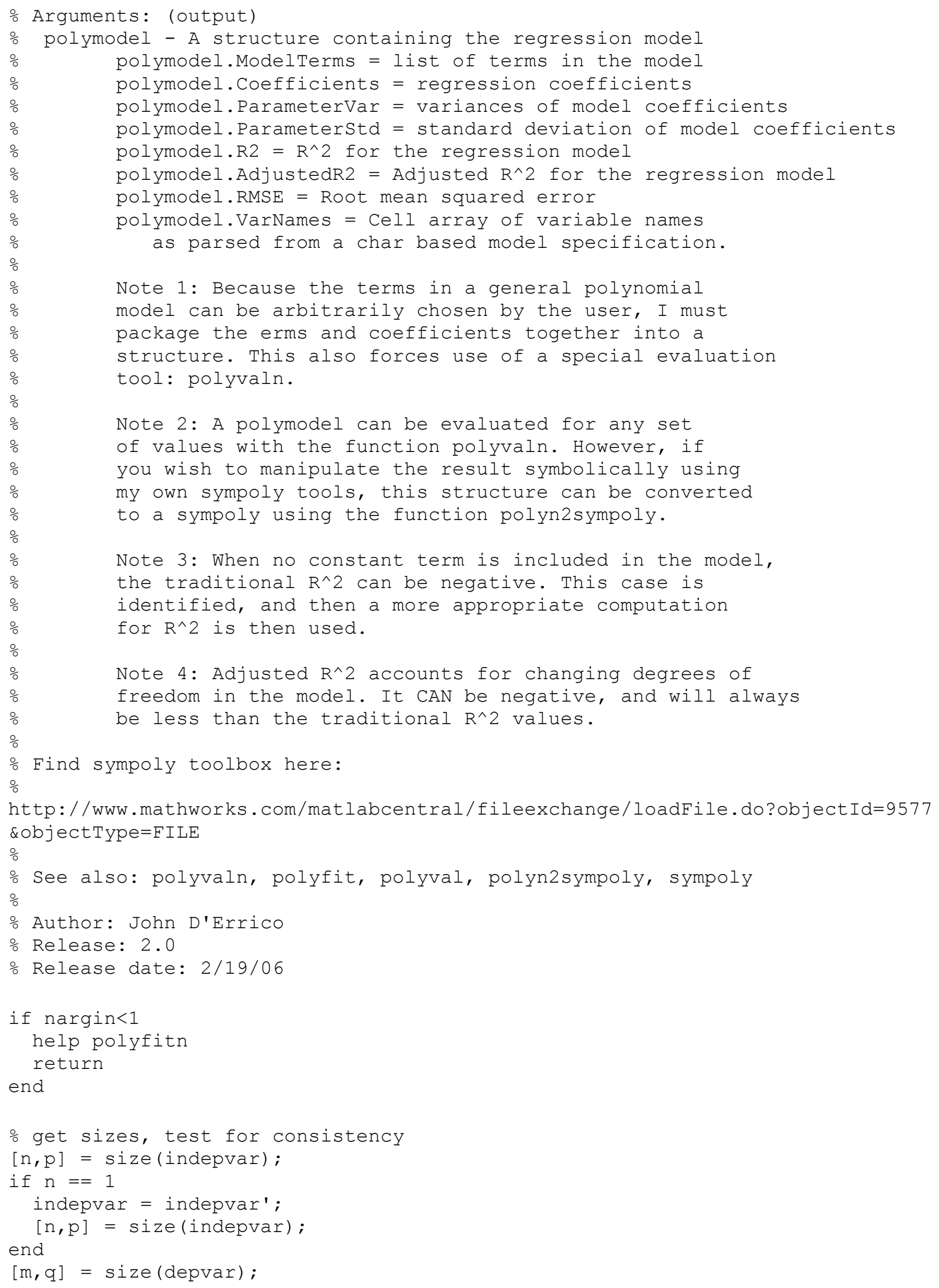




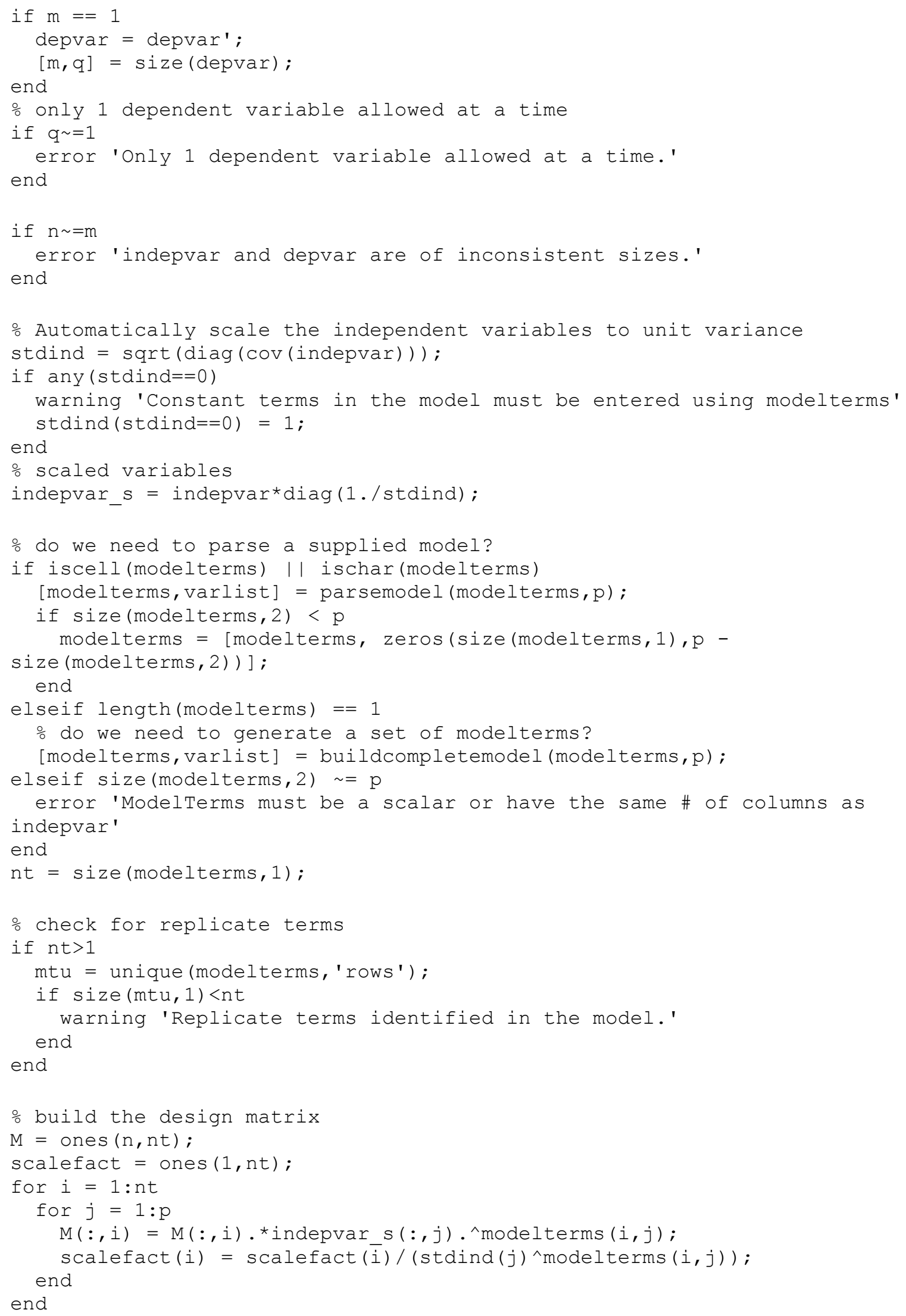




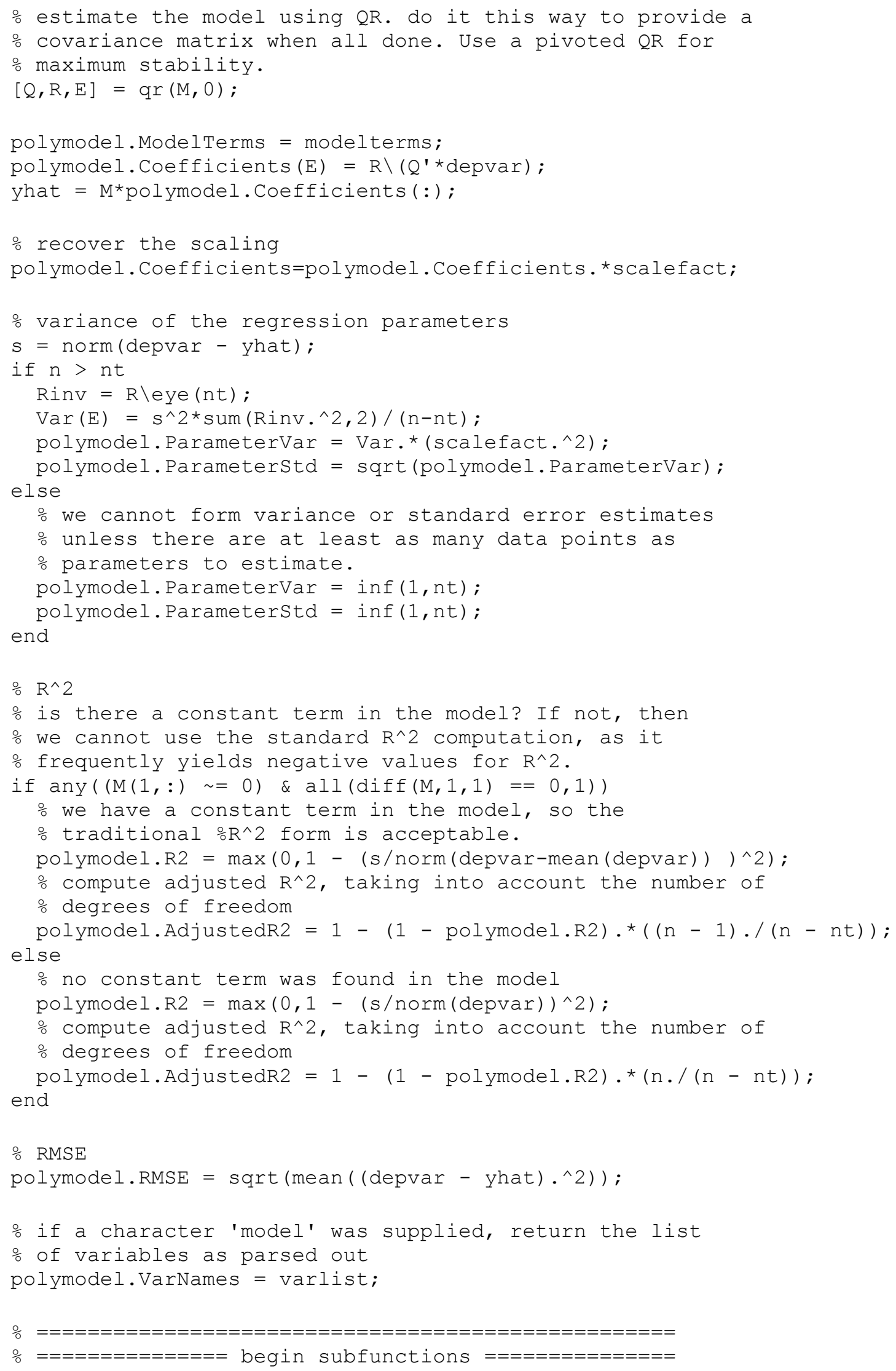




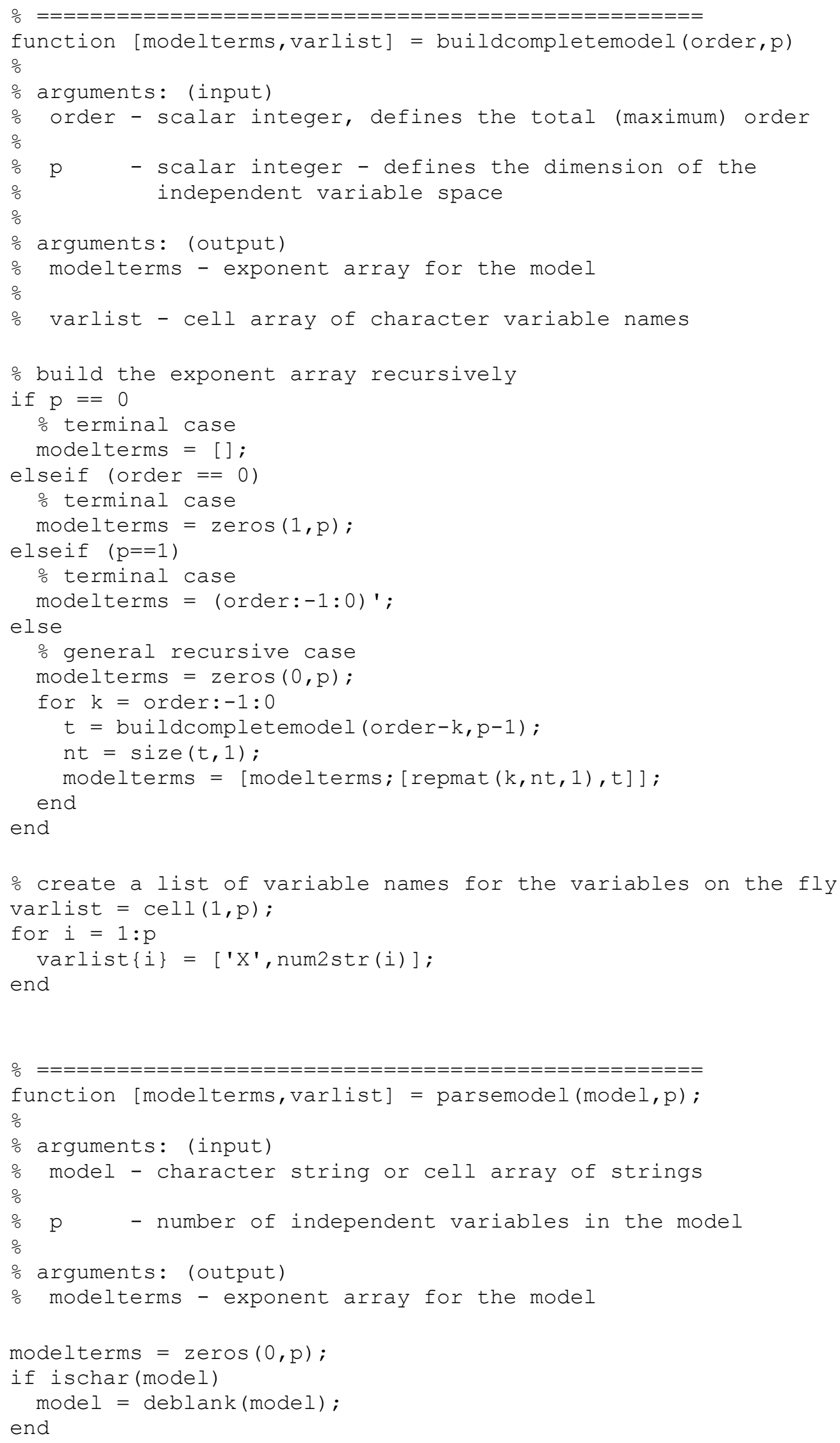




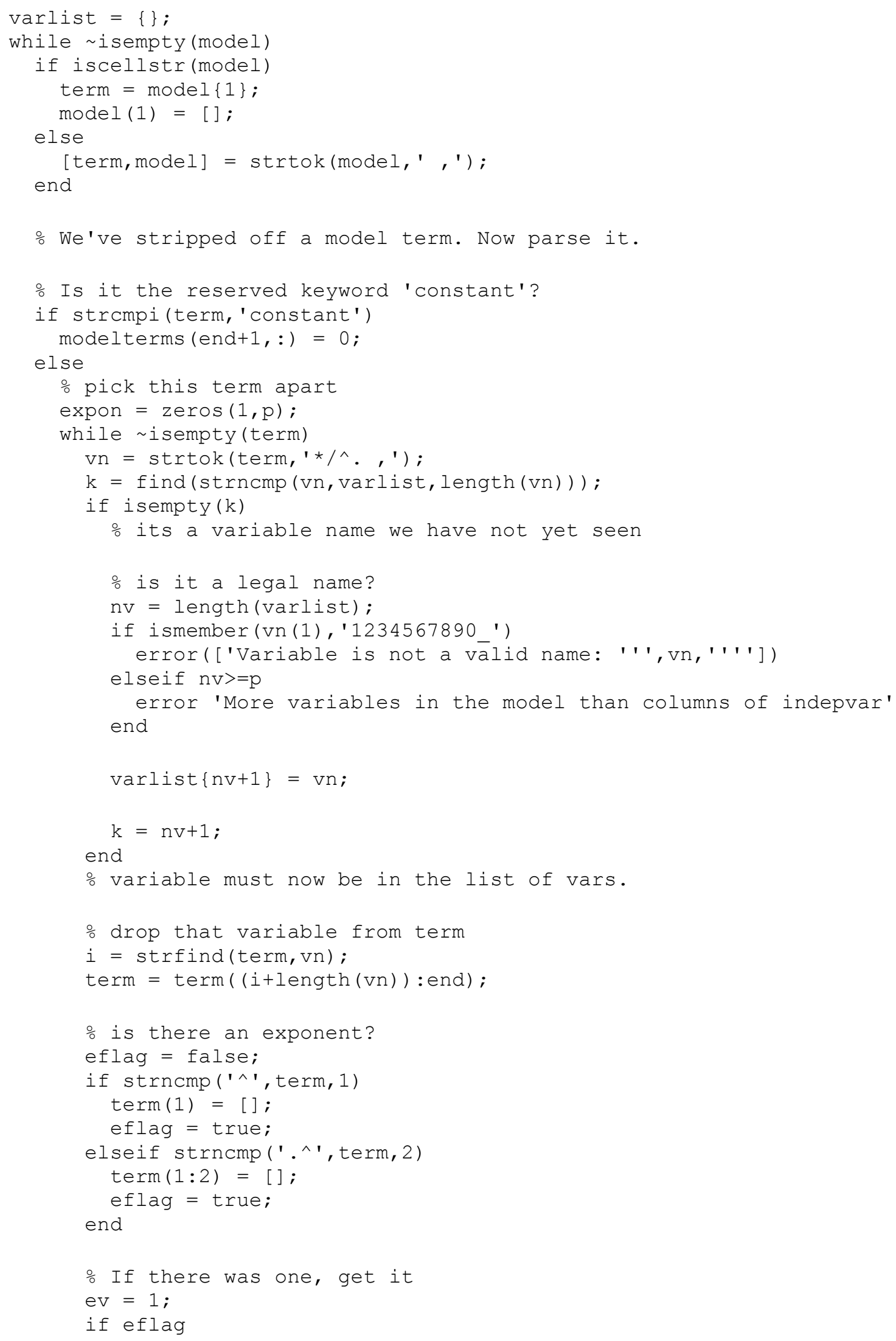




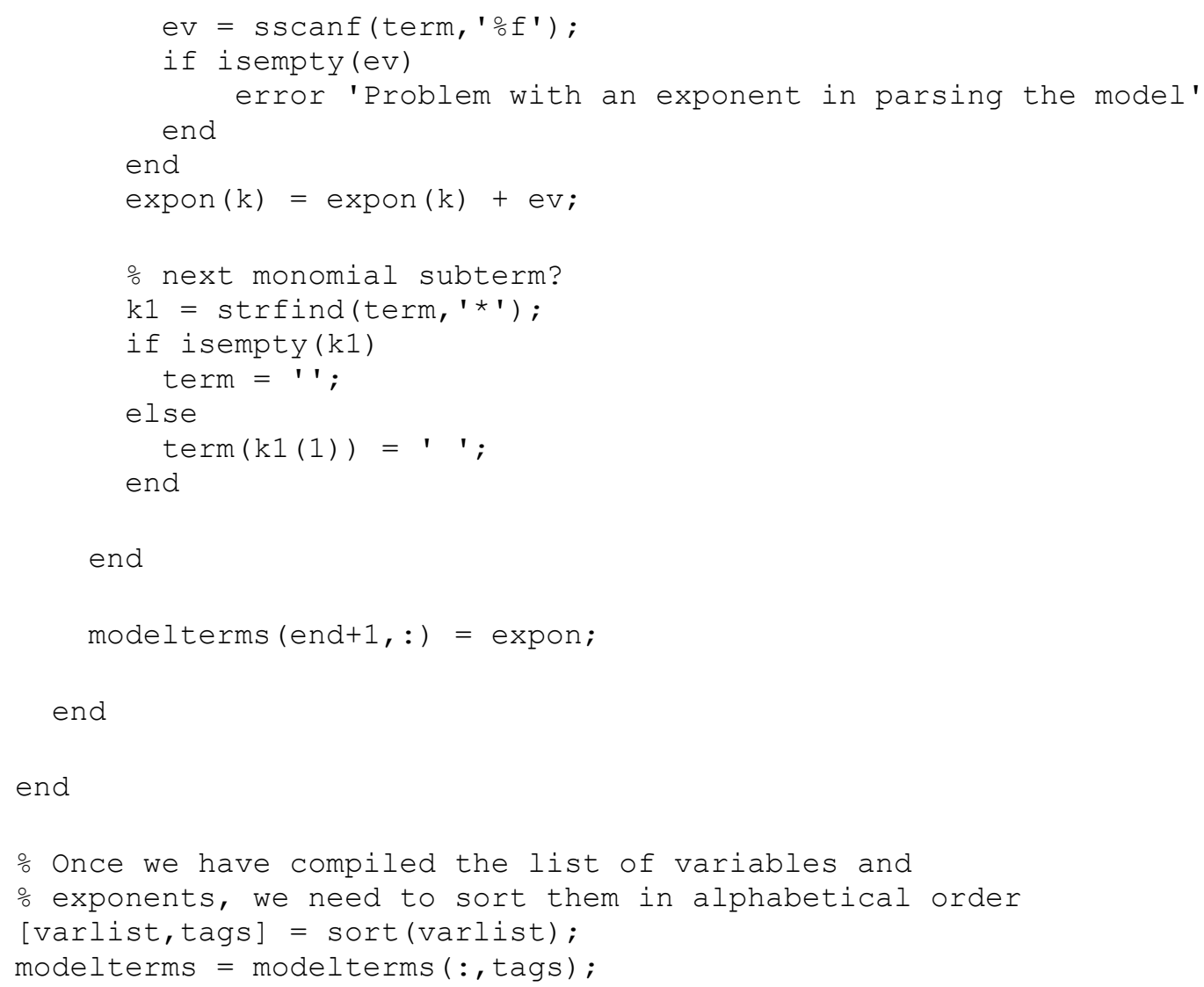

\section{A.8.15. Theoretical Maximum Bending Strength With Respect to Minimum Weight}

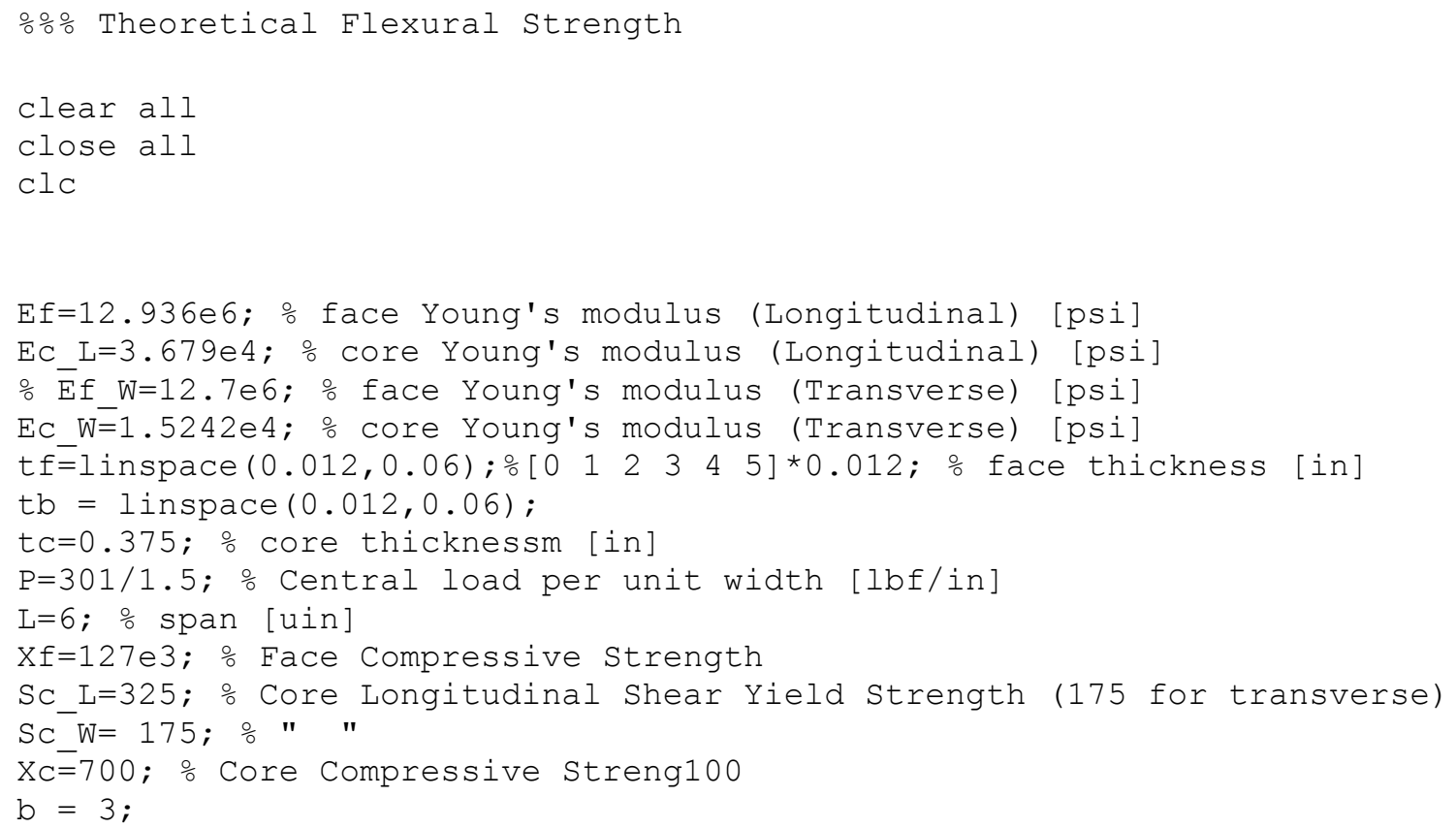




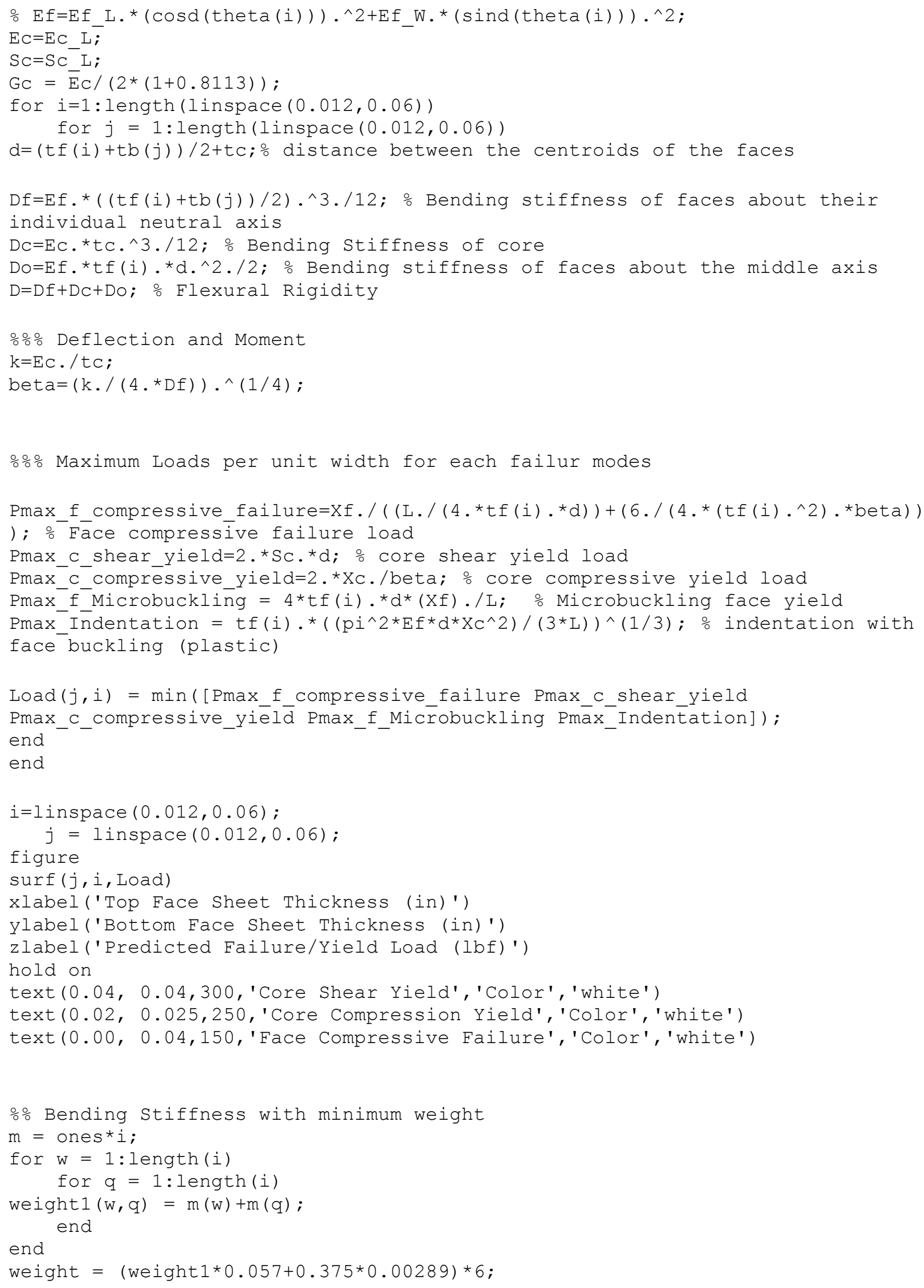


Ltow $=$ Load./Weight;

figure

surf (j,i, Load. /weight./min (Ltow (:)))

xlabel('Top Face Sheet Thickness (in)')

ylabel('Bottom Face Sheet Thickness (in)')

zlabel ('Normalised Strength/Weight (lbf)')

hold on

text $(0.04,0.04,6$, 'Core Shear Yield','Color', 'white')

text $(0.02,0.025,7$,' Core Compression Yield', 'Color', 'white')

text(0.00, 0.04,4,'Face Compressive Failure','Color', 'white')

$[$ ValueC, IndC $]=\max (\operatorname{LtoW}(:))$;

$[\mathrm{MC}, \mathrm{NC}]=$ ind2 sub ( size (Load. /weight), IndC) 